[Supporting Information]

\title{
$8 \pi$ Electrocyclic Reaction of Phosphonate Derivatives: Access to Seven-Membered Cross-Conjugated Cyclic Trienes
}

\author{
Hiroki Saito,${ }^{\ddagger}$ Ranmaru Kato,${ }^{\ddagger}$ Kazutada Ikeuchi, ${ }^{\dagger}$ Takahiro Suzuki,${ }^{\dagger}$ Keiji Tanino $*, \dagger$ \\ E-mail (Keiji Tanino*): ktanino@sci.hokudai.ac.jp \\ $¥$ Graduate School of Chemical Sciences and Engineering, Hokkaido University, Sapporo 060- \\ 0810, Japan \\ $\dagger$ Department of Chemistry, Faculty of Science, Hokkaido University, Sapporo 060-0810, Japan
}

\section{Table of Contents}

General Information

Experimental and Characterization Details

Outline for Preparations of Cyclization Precursors $\quad$ S3

$\begin{array}{ll}\text { Synthesis of Allyl Bromides } & \text { S3 }\end{array}$

Synthesis of cis-Cinnamaldehyde (S6) S5

General Procedure for Preparations of $\gamma, \delta$-Unsaturated Phosphonate S5

$\begin{array}{ll}\text { Synthesis of } \gamma, \delta \text {-Unsaturated Phosphonates } & \text { S6 }\end{array}$

General Procedure for Preparations of Cyclization Precursor $\quad$ S9

$\begin{array}{lr}\text { Synthesis of Cyclization Precursors } & \text { S10 }\end{array}$

Anionic $8 \pi$ Electrocyclic and Subsequent HWE Reactions of Stereoisomers

$\begin{array}{ll}\text { of Triene } 5 \text { (Scheme 3) } & \text { S18 }\end{array}$

General Procedure for $8 \pi$ Electrocyclization/HWE Reaction Sequence S19

$\begin{array}{ll}\text { Scope of Aldehydes in One-pot HWE Reactions (Figure 1) } & \text { S20 }\end{array}$

Reactions with Phosphonate Substrates Bearing Various Substituents (Figure 2) $\quad$ S24

Reactions of Trienes with Alkyl groups at Each Terminus (Scheme 4) S27

Synthesis and Reaction of a Substrate with an Oxygenated Functionality (Scheme 5) S29

$\begin{array}{ll}\text { References } & \text { S32 }\end{array}$

${ }^{1} \mathrm{H},{ }^{13} \mathrm{C}$, and ${ }^{31} \mathrm{P}$ NMR Spectra $\quad$ S33

$\begin{array}{ll}\text { Spectra for NOE experiments of } \mathbf{8 a} \text { and } \mathbf{1 8} & \text { S112 }\end{array}$

$\begin{array}{ll}\text { HMQC spectrum of } 7 & \text { S113 }\end{array}$ 


\section{General Information}

The reactions were performed using flame-dried glasswares under a positive pressure of argon. Oil bath was used when reactions required heating. Tetrahydrofuran (THF) was distilled from sodium benzophenone ketyl. Anhydrous dichloromethane and toluene were purchased from Kanto Chemical Co. Diisopropylamine was distilled from $\mathrm{CaH}_{2}$ under argon and stored in the presence of $\mathrm{NaOH}$ (pellets). 4,4-Dimethyl-2-((triisopropylsilyl)oxy)cyclopentane-1-carbonitrile $\quad(\mathbf{S 1})^{[1]}, \quad 1-$ (bromomethyl)cyclopent-1-ene ${ }^{[1]}$, 1-(bromomethyl)cyclohex-1-ene ${ }^{[2]}$, (E)-(3-bromoprop-1-en-1yl)cyclohexane ${ }^{[3]}, \quad(E)$-6-iodohex-3-en-1-ol $\quad$ tert-butyldimethylsilyl $\quad$ ether $^{[4]}$, and $(E)$-3cyclohexylpropenal ${ }^{[5]}$ were prepared by the known procedures.

Analytical thin layer chromatography (TLC) was performed using $0.25 \mathrm{~mm}$ Silica gel (60F-254) plates purchased from Merck Millipore. Reaction components were visualized by illumination with ultraviolet light $(254 \mathrm{~nm})$ and by staining with $8 \%$ ethanolic phosphomolybdic acid, ceric ammonium molybdate in $10 \%$ sulfuric acid, or basic potassium permanganate solution. Flash column chromatography was performed with Silica Gel 60N (neutral, particle size 40-50 $\mu \mathrm{m}$ ) purchased from Kanto Chemical Co., Inc. Preparative thin layer chromatography (PTLC) was performed using PLC Silica Gel 60 F254 purchased from Merck Millipore.

The melting points were determined using an AS ONE ATM-02 apparatus and uncorrected. ${ }^{1} \mathrm{H}(500$ $\mathrm{MHz}),{ }^{13} \mathrm{C}(126 \mathrm{MHz})$, and ${ }^{31} \mathrm{P}(202 \mathrm{MHz}) \mathrm{NMR}$ spectra were measured using a JEOL ECA-500. Chemical shifts are reported in parts per million (ppm) with internal $\mathrm{CDCl}_{3}\left({ }^{1} \mathrm{H} \mathrm{NMR}, \delta_{\mathrm{H}} 7.26 .{ }^{13} \mathrm{C}\right.$ NMR, $\left.\delta_{\mathrm{c}} 77.0\right)$ or external phosphoric acid $\left({ }^{31} \mathrm{P} \mathrm{NMR}, \delta_{\mathrm{P}} 0.00\right)$. Signals are expressed as broad (br), singlet (s), doublet (d), triplet (t), quartet (q), quintet (quin), septet (sept), and multiplet (m). Coupling constants are reported in Hz. IR spectra were recorded on JASCO FT/IR-4100 and the major absorbance bands are all reported in wavenumbers $\left(\mathrm{cm}^{-1}\right)$. High-resolution mass spectra (HRMS) were recorded on a JEOL JMS-T100GCV (GC-TOFMS). X-ray crystallographic analysis was conducted using a Rigaku XtaLAB Synergy-S. 


\section{Experimental and Characterization Details}

\section{Outline for Preparations of Cyclization Precursors}<smiles>CCCCOCCC=CCCI</smiles>

$\mathrm{HPO}(\mathrm{OEt})_{2}$ (2.0 equiv.) $\mathrm{NaH}$ (2.0 equiv.)

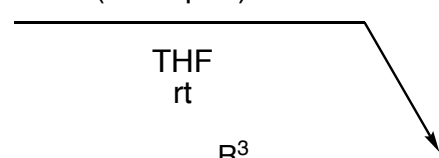<smiles>[R]C([R])=C([R])CBr</smiles><smiles>[R]C([R])=C([R])CCP(=O)(OCC)OCC</smiles>

4, 4a-4f, S7, S8

\begin{tabular}{ccccc} 
& $\mathrm{R}^{1}$ & $\mathrm{R}^{2}$ & $\mathrm{R}^{3}$ & $\mathrm{R}^{4}$ \\
\hline 4, 5 & $\mathrm{H}$ & $\mathrm{Ph}$ & $\mathrm{H}$ & $\mathrm{Ph}$ \\
4a, 10a & $\mathrm{Me}$ & $\mathrm{H}$ & $\mathrm{H}$ & $\mathrm{Ph}$ \\
4b, 10b & $\mathrm{H}$ & $\left(\mathrm{CH}_{2}\right)_{2} \mathrm{OTBS}$ & $\mathrm{H}$ & $\mathrm{Ph}$ \\
4c, 10c & $\mathrm{H}$ & $\mathrm{Me}$ & $\mathrm{Me}$ & $\mathrm{Ph}$ \\
4d, 10d & \multicolumn{2}{c}{$-\left(\mathrm{CH}_{2}\right)_{3^{-}}$} & $\mathrm{H}$ & $\mathrm{Ph}$ \\
4e, 10e & $-\mathrm{CH}_{2} \mathrm{CMe}_{2} \mathrm{CH}_{2^{-}}$ & $\mathrm{H}$ & $\mathrm{Ph}$ \\
4f, 10f & \multicolumn{2}{c}{$-\left(\mathrm{CH}_{2}\right)_{4^{-}}$} & $\mathrm{H}$ & $\mathrm{Ph}$ \\
S7, 12a & $\mathrm{H}$ & $\mathrm{Cy}$ & $\mathrm{H}$ & $\mathrm{Cy}$ \\
12b & $\mathrm{H}$ & $\mathrm{Cy}$ & $\mathrm{H}$ & $\mathrm{Me}$ \\
12c & $\mathrm{H}$ & $\mathrm{Cy}$ & $\mathrm{H}$ & ${ }^{n} \mathrm{Pr}$ \\
S8, 12d & $\mathrm{H}$ & ${ }^{n} \mathrm{Pr}$ & $\mathrm{H}$ & $\mathrm{Cy}$ \\
\hline
\end{tabular}

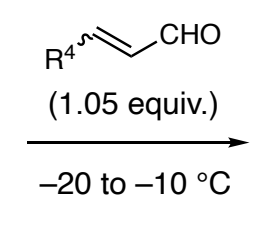<smiles></smiles>

$5,10 a-f, 12 a-d$

\section{Synthesis of Allyl Bromides}

\section{1-(Bromomethyl)-4,4-dimethylcyclopent-1-ene (S4)}
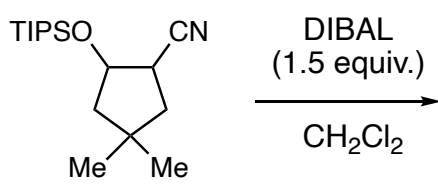

S1 dr = 1.1:1.0

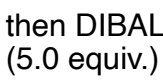

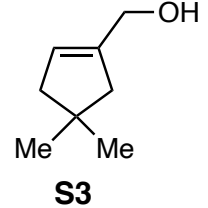

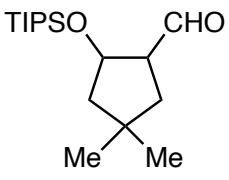

S2 $\mathrm{dr}=1.2: 1.0$
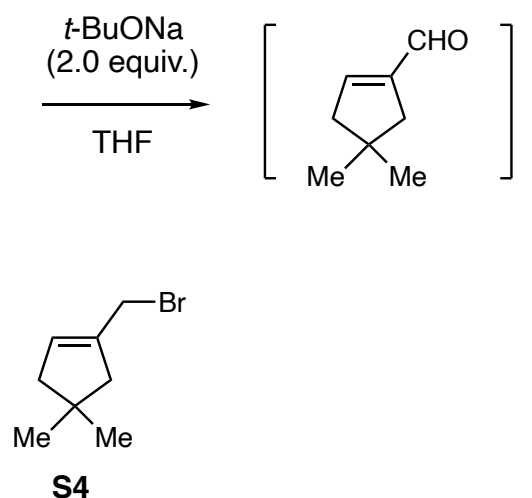

To a solution of 4,4-Dimethyl-2-((triisopropylsilyl)oxy)cyclopentane-1-carbonitrile (S1) $)^{[1]}(1.16 \mathrm{~g}$, $3.93 \mathrm{mmol}, \mathrm{dr}=1.1: 1.0)$ in $\mathrm{CH}_{2} \mathrm{Cl}_{2}(20 \mathrm{~mL})$ at $0{ }^{\circ} \mathrm{C}$ was added a $1.03 \mathrm{M}$ solution of DIBAL in $n$ hexane $(5.72 \mathrm{~mL}, 5.89 \mathrm{mmol})$ over $1 \mathrm{~min}$. After stirring for $1 \mathrm{~h}, 10 \%$ aqueous tartaric acid $(20 \mathrm{~mL})$ was added and then the mixture was vigorously stirred for $2.5 \mathrm{~h}$ at room temperature. The two layers 
were separated, and the aqueous layer was extracted with $\mathrm{Et}_{2} \mathrm{O}(3 \times 10 \mathrm{~mL})$. The combined organic layers were washed with brine $(20 \mathrm{~mL})$, dried over $\mathrm{MgSO}_{4}$, filtered, and concentrated under reduced pressure to afford crude $\beta$-siloxy aldehyde $\mathbf{S 2}$ as a 1.2:1.0 mixture of diastereomers.

The crude $\mathbf{S 2}$ was dissolved in THF (20 mL) and $t$-BuONa $(755 \mathrm{mg}, 7.86 \mathrm{mmol})$ was added at $0{ }^{\circ} \mathrm{C}$. The mixture was then allowed to warm to room temperature and stirred for $1 \mathrm{~h}$. Then, the mixture was cooled to $-78^{\circ} \mathrm{C}$, and a $1.03 \mathrm{M}$ solution of DIBAL in $n$-hexane $(19.1 \mathrm{~mL}, 19.7 \mathrm{mmol})$ was added over $2 \mathrm{~min}$. After stirring for $25 \mathrm{~min}, 10 \%$ aqueous tartaric acid $(20 \mathrm{~mL})$ was added, and then the mixture was vigorously stirred for $18 \mathrm{~h}$ at room temperature. The two layers were separated, and the aqueous layer was extracted with $\mathrm{Et}_{2} \mathrm{O}(3 \times 20 \mathrm{~mL})$. The combined organic layers were washed with brine $(20 \mathrm{~mL})$ dried over $\mathrm{MgSO}_{4}$, filtered, and concentrated under reduced pressure. Purification by flash column chromatography $\left(\mathrm{SiO}_{2}, \mathrm{Et}_{2} \mathrm{O} /\right.$ pentane $=1: 5$ to 1:3) afforded $\mathbf{S 3}$ accompanied with some impurities.

The obtained $\mathbf{S 3}$ was dissolved in $\mathrm{Et}_{2} \mathrm{O}(6.0 \mathrm{~mL})$ and $\mathrm{PBr}_{3}(133 \mu \mathrm{L}, 1.40 \mathrm{mmol})$ was added at $0{ }^{\circ} \mathrm{C}$. The reaction mixture was allowed to warm to room temperature and stirred for $0.5 \mathrm{~h}$. Then, the mixture was cooled to $0{ }^{\circ} \mathrm{C}$, and saturated aqueous $\mathrm{NaHCO}_{3}(3 \mathrm{~mL})$ was added. The two layers were separated, and the aqueous layer was extracted with $\mathrm{Et}_{2} \mathrm{O}(3 \times 3 \mathrm{~mL})$. The combined organic layers were dried over $\mathrm{MgSO}_{4}$, filtered, and concentrated under reduced pressure. Purification through a short pad of silica gel (pentane only) afforded S4 (267 mg, $1.41 \mathrm{mmol}, 36 \%$ from S1).

S4: colorless oil. ${ }^{1} \mathrm{H}$ NMR spectral property in $\mathrm{CDCl}_{3}$ was identical to that reported. ${ }^{[6]}$

\section{(E)-1-Bromohex-2-ene (S5)}

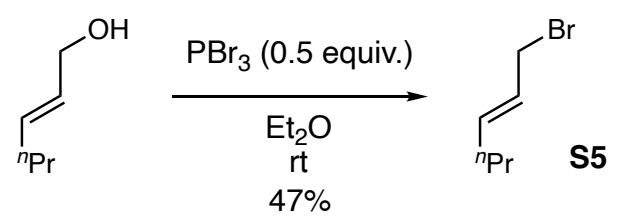

To a solution of $(E)$-2-hexene-1-ol $(590 \mu \mathrm{L}, 5.00 \mathrm{mmol})$ in $\mathrm{Et}_{2} \mathrm{O}(10 \mathrm{~mL})$ was slowly added $\mathrm{PBr}_{3}$ $(237 \mu \mathrm{L}, 2.50 \mathrm{mmol})$ at $0{ }^{\circ} \mathrm{C}$. The reaction mixture was allowed to warm to room temperature and stirred for $50 \mathrm{~min}$. Then, to the mixture was added saturated aqueous $\mathrm{NaHCO}_{3}(10 \mathrm{~mL})$. The two layers were separated, and the aqueous layer was extracted with $\mathrm{Et}_{2} \mathrm{O}(3 \times 5 \mathrm{~mL})$. The combined organic layers were dried over $\mathrm{MgSO}_{4}$, filtered, and concentrated under reduced pressure. Purification through a short pad of silica gel ( $n$-hexane only) afforded S5 (380 mg, $2.33 \mathrm{mmol}, 47 \%)$.

S5: colorless oil. ${ }^{1} \mathrm{H}$ NMR spectral property in $\mathrm{CDCl}_{3}$ was identical to that reported. ${ }^{[7]}$ 


\section{Synthesis of cis-Cinnamaldehyde (S6)}

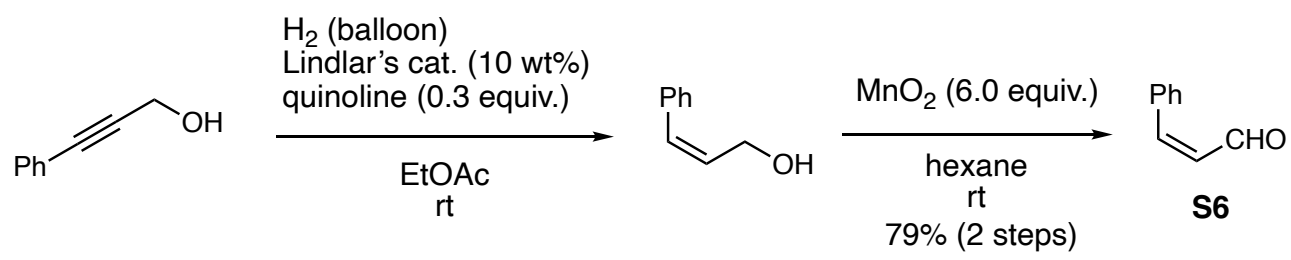

To a solution of 3-phenyl-2-propyn-1-ol $(374 \mu \mathrm{L}, 3.00 \mathrm{mmol})$ in EtOAc $(7.5 \mathrm{~mL})$ were added quinoline (107 $\mu \mathrm{L}, 0.90 \mathrm{mmol})$ and Lindlar's catalyst (38 mg, $10 \mathrm{wt} \%$, Kawaken Fine Chemical Co., Ltd.). A balloon filled with hydrogen gas was attached to the reaction vessel, and the reaction atmosphere was replaced with hydrogen gas. The reaction mixture was stirred at room temperature for $1 \mathrm{~h}$. Then, the mixture was filtered through a pad of Celite ${ }^{\circledR}$, and the filter cake was washed with EtOAc $(2 \mathrm{~mL})$. The filtrate was washed with $1 \mathrm{M}$ aqueous $\mathrm{HCl}(3 \times 2 \mathrm{~mL})$. The organic layer was dried over $\mathrm{MgSO}_{4}$ and concentrated under reduced pressure to give a crude alcohol.

To a solution of the crude alcohol in $n$-hexane $(60 \mathrm{~mL})$ was added $\mathrm{MnO}_{2}(1.56 \mathrm{~g}, 17.9 \mathrm{mmol})$. After stirring for $14.5 \mathrm{~h}$ in dark, the reaction mixture was filtered through a pad of Celite ${ }^{\circledR}$, and the filter cake was washed with $n$-hexane. The filtrate was concentrated under reduced pressure. Purification by flash column chromatography $\left(\mathrm{SiO}_{2}, n\right.$-hexane/EtOAc $\left.=50: 1\right)$ afforded $\mathbf{S 6}$ (315 mg, $2.38 \mathrm{mmol}$, $79 \%$ for 2 steps).

S6: pale yellow oil. ${ }^{1} \mathrm{H}$ NMR spectral property in $\mathrm{CDCl}_{3}$ was identical to that reported. ${ }^{[8]}$

\section{General Procedure for Synthesis of $\gamma, \delta$-Unsaturated Phosphonate}

\section{Procedure A}

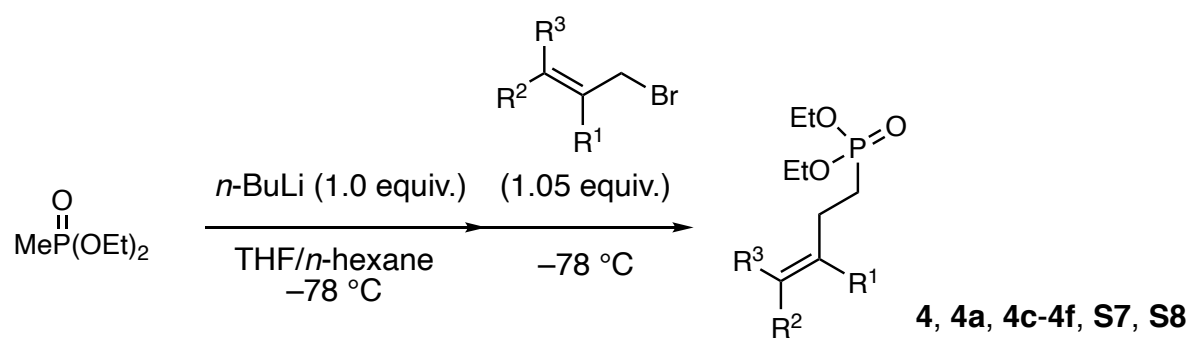

To a solution of diethyl methylphosphonate (1.00 equiv.) in THF $(0.2 \mathrm{M})$ at $-78{ }^{\circ} \mathrm{C}$ was slowly added a solution of $n$-BuLi in $n$-hexane (1.00 equiv.). After 20 min, allyl bromide (1.05 equiv.) was quickly added, and resulting mixture was stirred for $0.5 \sim 1.5 \mathrm{~h}$ at $-78{ }^{\circ} \mathrm{C}$. Then, the reaction was quenched with saturated aqueous $\mathrm{NH}_{4} \mathrm{Cl}$. EtOAc was added, and the two layers were separated, and the aqueous layer was extracted with EtOAc (2 times). The combined organic layers were dried over $\mathrm{MgSO}_{4}$, filtered, and concentrated under reduced pressure. Purification by silica gel flash column chromatography (EtOAc only) afforded $\gamma, \delta$-unsaturated phosphonate. 


\section{Synthesis of $\gamma, \delta$-Unsaturated Phosphonates}

\section{Diethyl (E)-(4-phenylbut-3-en-1-yl)phosphonate (4)}

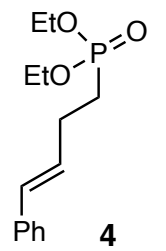

Phosphonate 4 (1.19 g, 4.44 mmol, 89\%) was obtained from diethyl methylphosphonate $(722 \mu \mathrm{L}, 5.00 \mathrm{mmol})$, a $2.67 \mathrm{M}$ solution of $n$-BuLi in $n$-hexane $(1.87 \mathrm{~mL}, 5.00 \mathrm{mmol})$, and cinnamyl bromide $(1.03 \mathrm{~g}, 5.25 \mathrm{mmol})$ according to Procedure A (reaction time: 50 $\min )$.

4: colorless oil. ${ }^{1} \mathrm{H}$ NMR $\left(500 \mathrm{MHz}, \mathrm{CDCl}_{3}\right) \delta 7.36-7.27(\mathrm{~m}, 4 \mathrm{H}), 7.21(\mathrm{dd}, J=7.2,7.2 \mathrm{~Hz}, 1 \mathrm{H})$, $6.43(\mathrm{~d}, J=16.1 \mathrm{~Hz}, 1 \mathrm{H}), 6.22(\mathrm{dt}, J=15.5,6.9 \mathrm{~Hz}, 1 \mathrm{H}), 4.17-4.06(\mathrm{~m}, 4 \mathrm{H}), 2.57-2.46(\mathrm{~m}, 2 \mathrm{H})$, $1.96-1.86(\mathrm{~m}, 2 \mathrm{H}), 1.33(\mathrm{t}, J=7.2 \mathrm{~Hz}, 6 \mathrm{H}) .{ }^{13} \mathrm{C} \mathrm{NMR}\left(126 \mathrm{MHz}, \mathrm{CDCl}_{3}\right) \delta 137.2,130.6,128.9(\mathrm{~d}$, $\left.J_{\mathrm{C}-\mathrm{P}}=16.8 \mathrm{~Hz}\right), 128.5(2 \mathrm{C}), 127.2,126.0(2 \mathrm{C}), 61.5\left(\mathrm{~d}, J_{\mathrm{C}-\mathrm{P}}=6.0 \mathrm{~Hz}, 2 \mathrm{C}\right), 26.0\left(\mathrm{~d}, J_{\mathrm{C}-\mathrm{P}}=3.6 \mathrm{~Hz}\right)$, $25.5\left(\mathrm{~d}, J_{\mathrm{C}-\mathrm{P}}=139 \mathrm{~Hz}\right), 16.5\left(\mathrm{~d}, J_{\mathrm{C}-\mathrm{P}}=6.0 \mathrm{~Hz}, 2 \mathrm{C}\right) .{ }^{31} \mathrm{P} \mathrm{NMR}\left(202 \mathrm{MHz}, \mathrm{CDCl}_{3}\right) \delta$ 31.9. IR (ATR) $v$ 2981, 1240, 1163, 1024, $958 \mathrm{~cm}^{-1}$. HRMS (FD) $m / z$ : [M] $]^{+}$calcd for $\mathrm{C}_{14} \mathrm{H}_{21} \mathrm{O}_{3} \mathrm{P} 268.1228$; found: 268.1218 .

\section{Diethyl (3-methylbut-3-en-1-yl)phosphonate (4a)}

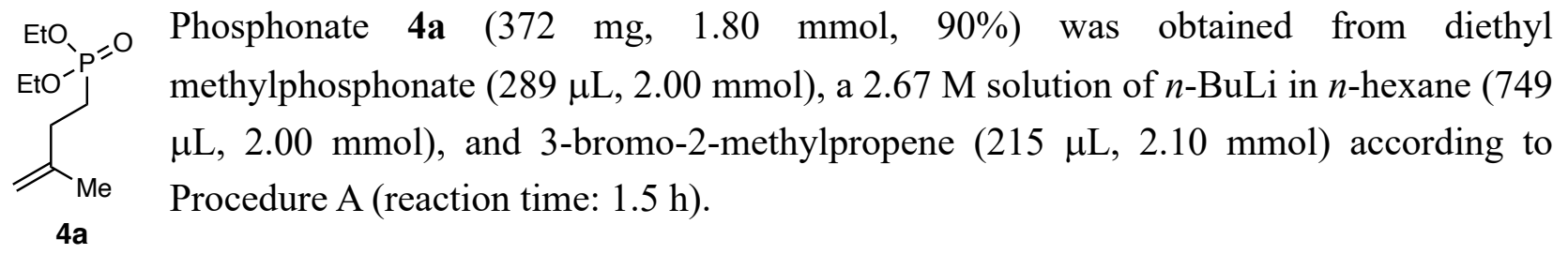

4a: colorless oil. ${ }^{1} \mathrm{H}$ NMR spectral property in $\mathrm{CDCl}_{3}$ was identical to that reported. ${ }^{[9]}$

\section{Diethyl (4-methylpent-3-en-1-yl)phosphonate (4c)}

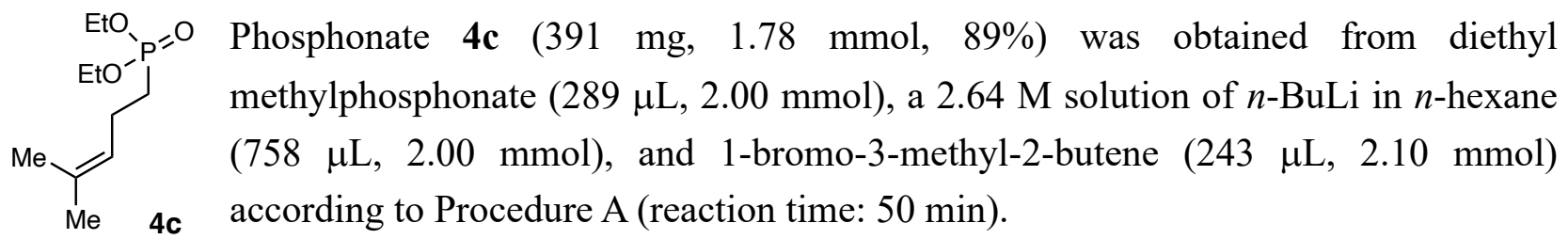

4c: colorless oil. ${ }^{1} \mathrm{H}$ NMR spectral property in $\mathrm{CDCl}_{3}$ was identical to that reported. ${ }^{[9]}$ 


\section{Diethyl (2-(cyclopent-1-en-1-yl)ethyl)phosphonate (4d)}

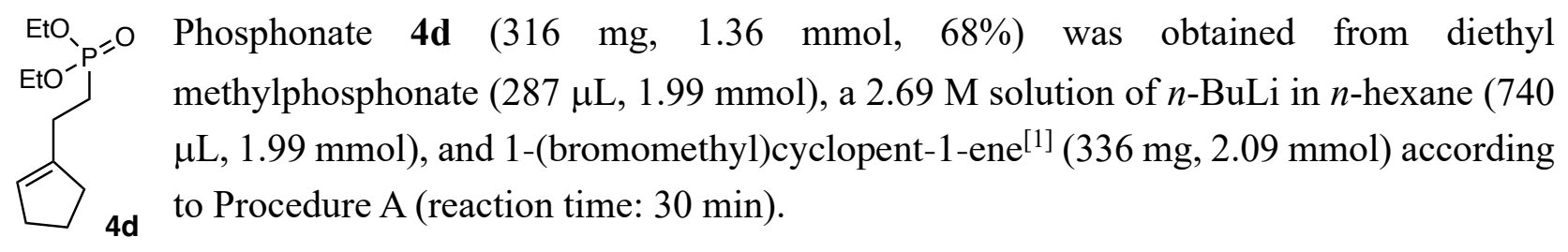

4d: colorless oil. ${ }^{1} \mathrm{H}$ NMR $\left(500 \mathrm{MHz}, \mathrm{CDCl}_{3}\right) \delta 5.36(\mathrm{~s}, 1 \mathrm{H}), 4.15-4.04(\mathrm{~m}, 4 \mathrm{H}), 2.39-2.26(\mathrm{~m}, 4 \mathrm{H})$, 2.24 (br t, $J=6.6 \mathrm{~Hz}, 2 \mathrm{H}), 1.94-1.83(\mathrm{~m}, 4 \mathrm{H}), 1.32$ (t, $J=6.9 \mathrm{~Hz}, 6 \mathrm{H}) .{ }^{13} \mathrm{C} \mathrm{NMR}\left(126 \mathrm{MHz}, \mathrm{CDCl}_{3}\right)$ $\delta 143.3\left(\mathrm{~d}, J_{\mathrm{C}-\mathrm{P}}=18.0 \mathrm{~Hz}\right), 123.8,61.4\left(\mathrm{~d}, J_{\mathrm{C}-\mathrm{P}}=7.2 \mathrm{~Hz}, 2 \mathrm{C}\right), 34.8,32.3,24.2\left(\mathrm{~d}, J_{\mathrm{C}-\mathrm{P}}=140 \mathrm{~Hz}\right)$, $23.9\left(\mathrm{~d}, J_{\mathrm{C}-\mathrm{P}}=4.8 \mathrm{~Hz}\right), 23.3,16.4\left(\mathrm{~d}, J_{\mathrm{C}-\mathrm{P}}=6.0 \mathrm{~Hz}, 2 \mathrm{C}\right) .{ }^{31} \mathrm{P} \mathrm{NMR}\left(202 \mathrm{MHz}, \mathrm{CDCl}_{3}\right) \delta 32.7 . \mathrm{IR}$ $\left(\text { ATR) } v 2979,1242,1056,1025,956 \mathrm{~cm}^{-1} \text {. HRMS (FD) } \mathrm{m} / z \text { : [M] }\right]^{+}$calcd for $\mathrm{C}_{11} \mathrm{H}_{21} \mathrm{O}_{3} \mathrm{P} 232.1228$; found: 232.1222 .

\section{Diethyl (2-(4,4-dimethylcyclopent-1-en-1-yl)ethyl)phosphonate (4e)}

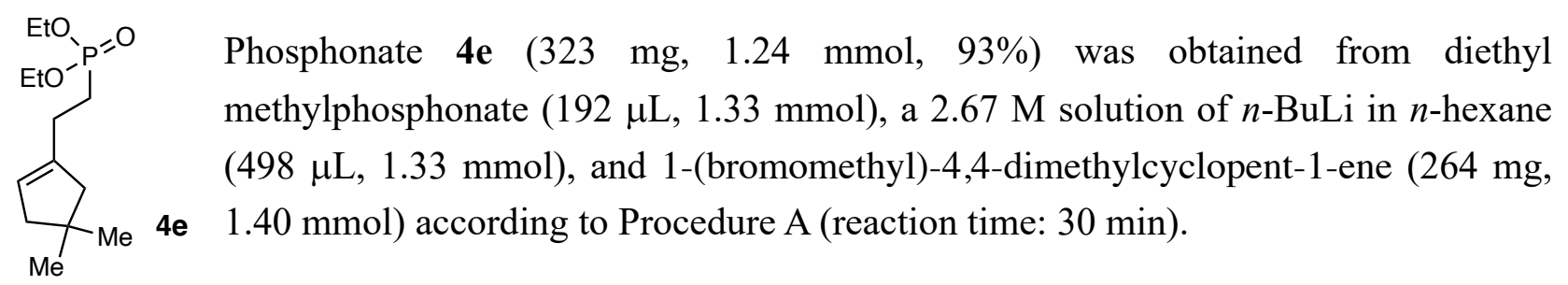

4e: colorless oil. ${ }^{1} \mathrm{H}$ NMR (500 MHz, $\left.\mathrm{CDCl}_{3}\right) \delta 5.23(\mathrm{~s}, 1 \mathrm{H}), 4.16-4.04$ (m, 4H), 2.29 (br ddd, $J=$ $8.3,8.3,8.3 \mathrm{~Hz}, 2 \mathrm{H}), 2.11(\mathrm{dd}, J=4.0,2.3 \mathrm{~Hz}, 2 \mathrm{H}), 2.05(\mathrm{~s}, 2 \mathrm{H}), 1.91-1.83(\mathrm{~m}, 2 \mathrm{H}), 1.33$ (t, $J=7.2$ $\mathrm{Hz}, 6 \mathrm{H}), 1.06(\mathrm{~s}, 6 \mathrm{H}) .{ }^{13} \mathrm{C} \mathrm{NMR}\left(126 \mathrm{MHz}, \mathrm{CDCl}_{3}\right) \delta 141.6\left(\mathrm{~d}, J_{\mathrm{C}-\mathrm{P}}=18.0 \mathrm{~Hz}\right), 122.3,61.2\left(\mathrm{~d}, J_{\mathrm{C}-\mathrm{P}}\right.$ $=7.2 \mathrm{~Hz}, 2 \mathrm{C}), 49.8,47.4,38.3,29.7(2 \mathrm{C}), 24.0\left(\mathrm{~d}, J_{\mathrm{C}-\mathrm{P}}=4.8 \mathrm{~Hz}\right), 23.9\left(\mathrm{~d}, J_{\mathrm{C}-\mathrm{P}}=140 \mathrm{~Hz}\right), 16.3(\mathrm{~d}$, $\left.J_{\mathrm{C}-\mathrm{P}}=6.0 \mathrm{~Hz}, 2 \mathrm{C}\right) .{ }^{31} \mathrm{P} \mathrm{NMR}\left(202 \mathrm{MHz}, \mathrm{CDCl}_{3}\right) \delta 32.8 . \mathrm{IR}(\mathrm{ATR}) \vee 2952,1244,1056,1027,960$ $\mathrm{cm}^{-1}$. HRMS (FD) $m / z$ : [M] $]^{+}$calcd for $\mathrm{C}_{13} \mathrm{H}_{25} \mathrm{O}_{3} \mathrm{P} 260.1541$; found: 260.1543 .

\section{Diethyl (2-(cyclohex-1-en-1-yl)ethyl)phosphonate (4f)}

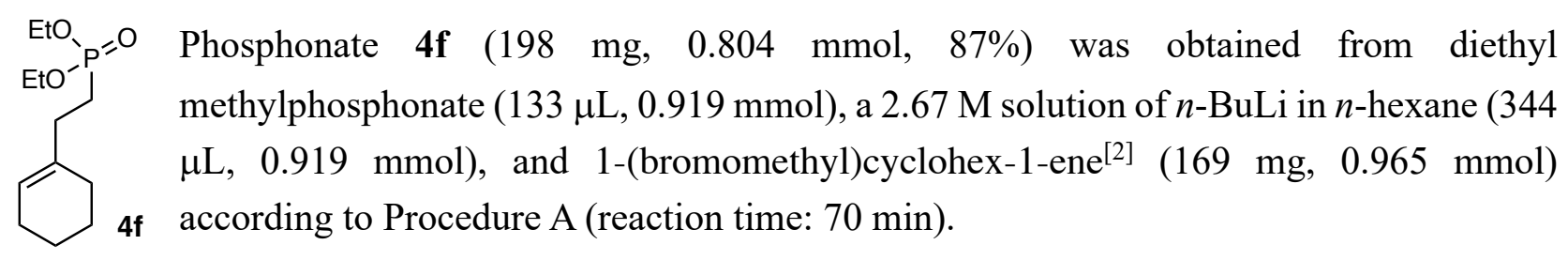

4f: colorless oil. ${ }^{1} \mathrm{H}$ NMR $\left(500 \mathrm{MHz}, \mathrm{CDCl}_{3}\right) \delta 5.44(\mathrm{~s}, 1 \mathrm{H}), 4.15-4.03(\mathrm{~m}, 4 \mathrm{H}), 2.20$ (br ddd, $J=$ 8.6, 8.6, 8.6 Hz, 2H), 2.01-1.95 (m, 2H), 1.91 (br, 2H), 1.87-1.79 (m, 2H), 1.65-1.51 (m, 4H), 1.32 $(\mathrm{t}, J=7.2 \mathrm{~Hz}, 6 \mathrm{H}) .{ }^{13} \mathrm{C} \mathrm{NMR}\left(126 \mathrm{MHz}, \mathrm{CDCl}_{3}\right) \delta 136.2\left(\mathrm{~d}, J_{\mathrm{C}-\mathrm{P}}=16.8 \mathrm{~Hz}\right), 121.1,61.2\left(\mathrm{~d}, J_{\mathrm{C}-\mathrm{P}}=\right.$ $7.2 \mathrm{~Hz}, 2 \mathrm{C}), 30.1\left(\mathrm{~d}, J_{\mathrm{C}-\mathrm{P}}=3.6 \mathrm{~Hz}\right), 27.8,24.9,23.9\left(\mathrm{~d}, J_{\mathrm{C}-\mathrm{P}}=139 \mathrm{~Hz}\right), 22.6,22.2,16.2\left(\mathrm{~d}, J_{\mathrm{C}-\mathrm{P}}=\right.$ 
$6.0 \mathrm{~Hz}, 2 \mathrm{C}) .{ }^{31} \mathrm{P}$ NMR $\left(202 \mathrm{MHz}, \mathrm{CDCl}_{3}\right) \delta 33.1$. IR (ATR) v 2926, 1245, 1027, $961 \mathrm{~cm}^{-1}$. HRMS (FD) $m / z:[\mathrm{M}]^{+}$calcd for $\mathrm{C}_{12} \mathrm{H}_{23} \mathrm{O}_{3} \mathrm{P} 246.1385$; found: 246.1379 .

\section{Diethyl (E)-(4-cyclohexylbut-3-en-1-yl)phosphonate (S7)}

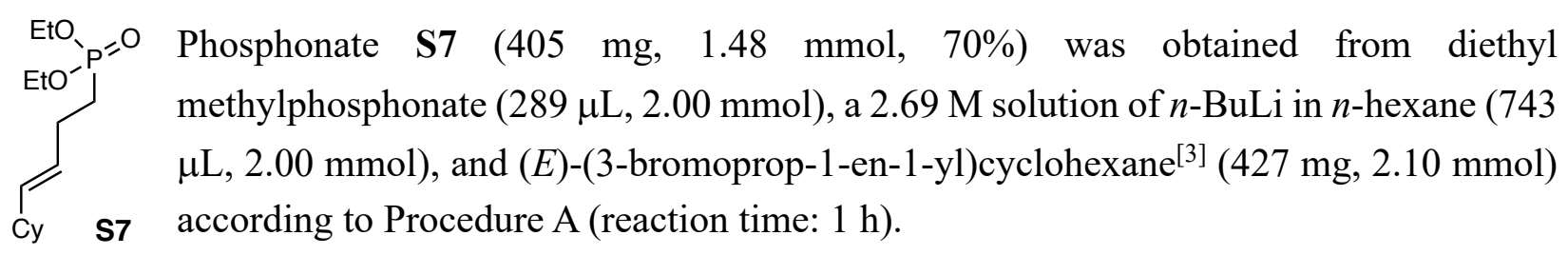

S7: pale yellow oil. ${ }^{1} \mathrm{H}$ NMR (500 MHz, $\left.\mathrm{CDCl}_{3}\right) \delta$ 5.44-5.34 (m, 2H), 4.14-4.04 (m, 4H), 2.31$2.23(\mathrm{~m}, 2 \mathrm{H}), 1.93-1.84(\mathrm{~m}, 1 \mathrm{H}), 1.82-1.74(\mathrm{~m}, 2 \mathrm{H}), 1.73-1.64(\mathrm{~m}, 4 \mathrm{H}), 1.32(\mathrm{t}, J=6.9 \mathrm{~Hz}, 6 \mathrm{H})$, $1.29-1.19(\mathrm{~m}, 3 \mathrm{H}), 1.14(\mathrm{tt}, J=12.3,2.9 \mathrm{~Hz}, 1 \mathrm{H}), 1.08-0.98(\mathrm{~m}, 2 \mathrm{H}) .{ }^{13} \mathrm{C}$ NMR $\left(126 \mathrm{MHz}, \mathrm{CDCl}_{3}\right)$ $\delta 137.2,125.9\left(\mathrm{~d}, J_{\mathrm{C}-\mathrm{P}}=18.0 \mathrm{~Hz}\right), 61.3\left(\mathrm{~d}, J_{\mathrm{C}-\mathrm{P}}=7.2 \mathrm{~Hz}, 2 \mathrm{C}\right), 40.4,32.9,26.3,26.1,25.9,25.41$, 25.37, 25.2, $16.4\left(\mathrm{~d}, J_{\mathrm{C}-\mathrm{P}}=6.0 \mathrm{~Hz}, 2 \mathrm{C}\right) .{ }^{31} \mathrm{P} \mathrm{NMR}\left(202 \mathrm{MHz}, \mathrm{CDCl}_{3}\right) \delta 32.5$. IR (ATR) v 2923, 1240, $1025,961 \mathrm{~cm}^{-1}$. HRMS (FD) $\mathrm{m} / z$ : [M] $]^{+}$calcd for $\mathrm{C}_{14} \mathrm{H}_{27} \mathrm{O}_{3} \mathrm{P} 274.1698$; found: 274.1700 .

\section{Diethyl (E)-(hept-3-en-1-yl)phosphonate (S8)}

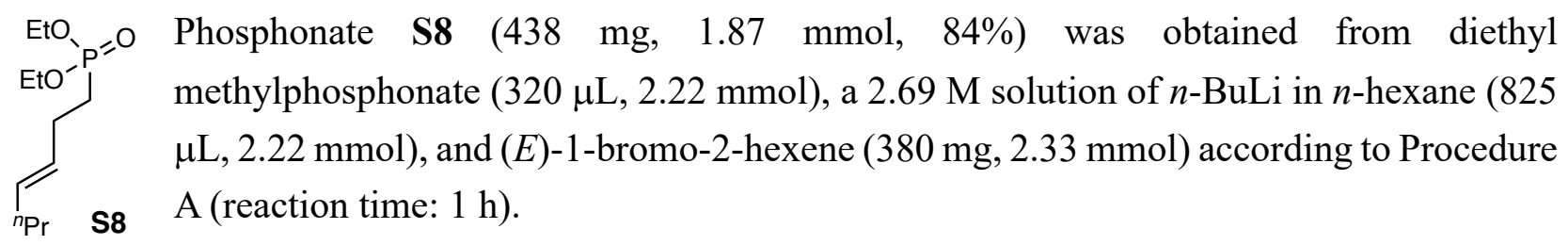

S8: pale yellow oil. ${ }^{1} \mathrm{H}$ NMR (500 MHz, $\left.\mathrm{CDCl}_{3}\right) \delta 5.50-5.38(\mathrm{~m}, 2 \mathrm{H}), 4.14-4.04(\mathrm{~m}, 4 \mathrm{H}), 2.32-$ $2.24(\mathrm{~m}, 2 \mathrm{H}), 1.96(\mathrm{q}, J=6.9 \mathrm{~Hz}, 2 \mathrm{H}), 1.84-1.74(\mathrm{~m}, 2 \mathrm{H}), 1.39-1.30(\mathrm{~m}, 8 \mathrm{H}), 0.88$ (t, $J=6.9 \mathrm{~Hz}$, $3 \mathrm{H}) .{ }^{13} \mathrm{C} \mathrm{NMR}\left(126 \mathrm{MHz}, \mathrm{CDCl}_{3}\right) \delta 131.1,128.7\left(\mathrm{~d}, J_{\mathrm{C}-\mathrm{P}}=18.0 \mathrm{~Hz}\right), 61.3\left(\mathrm{~d}, J_{\mathrm{C}-\mathrm{P}}=7.2 \mathrm{~Hz}, 2 \mathrm{C}\right)$, $34.4,25.7\left(\mathrm{~d}, J_{\mathrm{C}-\mathrm{P}}=140 \mathrm{~Hz}\right), 25.4\left(\mathrm{~d}, J_{\mathrm{C}-\mathrm{P}}=3.6 \mathrm{~Hz}\right), 22.4,16.3\left(\mathrm{~d}, J_{\mathrm{C}-\mathrm{P}}=6.0 \mathrm{~Hz}, 2 \mathrm{C}\right), 13.5 .{ }^{31} \mathrm{P} \mathrm{NMR}$ $\left(202 \mathrm{MHz}, \mathrm{CDCl}_{3}\right) \delta 32.4$. IR (ATR) $\vee 2958,1244,956,791 \mathrm{~cm}^{-1}$. HRMS (FD) $\mathrm{m} / z$ : [M] $]^{+}$calcd for $\mathrm{C}_{11} \mathrm{H}_{23} \mathrm{O}_{3} \mathrm{P}$ 234.1385; found: 234.1389. 


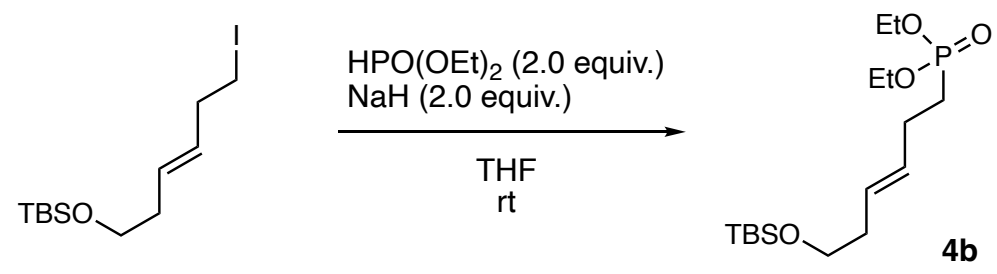

$55 \% \mathrm{NaH}$ in paraffin $(87.3 \mathrm{mg}, 2.00 \mathrm{mmol})$ placed in a flask was washed with $n$-hexane $(2 \mathrm{~mL})$ under argon atmosphere, and then THF $(1.0 \mathrm{~mL})$ was added. To the resulting suspension cooled in an ice bath was slowly added diethyl phosphite $(252 \mu \mathrm{L}, 2.00 \mathrm{mmol})$ and then the mixture was warmed to room temperature. After stirring for $1 \mathrm{~h}$, a solution of $(E)$-6-iodohex-3-en-1-ol tertbutyldimethylsilyl ether ${ }^{[4]}(340 \mathrm{mg}, 1.00 \mathrm{mmol})$ in THF $(2 \times 0.50 \mathrm{~mL})$ was slowly added at room temperature. After stirring for $15 \mathrm{~h}$ at room temperature, the mixture was cooled in an ice bath and saturated aqueous $\mathrm{NH}_{4} \mathrm{Cl}$ was added $(2 \mathrm{~mL})$. EtOAc $(2 \mathrm{~mL})$ was added, and the two layers were separated, and the aqueous layer was extracted with EtOAc $(2 \times 2 \mathrm{~mL})$. The combined organic layers were dried over $\mathrm{MgSO}_{4}$, filtered, and concentrated under reduced pressure. Purification by silica gel flash column chromatography afforded phosphonate $\mathbf{4 b}$ (258 mg, $0.735 \mathrm{mmol}, 74 \%$ ).

4b: pale yellow oil. ${ }^{1} \mathrm{H}$ NMR $\left(500 \mathrm{MHz}, \mathrm{CDCl}_{3}\right) \delta 5.53-5.43(\mathrm{~m}, 2 \mathrm{H}), 4.14-4.04(\mathrm{~m}, 4 \mathrm{H}), 3.61(\mathrm{t}, J$ $=6.9 \mathrm{~Hz}, 2 \mathrm{H}), 2.33-2.25(\mathrm{~m}, 2 \mathrm{H}), 2.21(\mathrm{dt}, J=6.3,6.3 \mathrm{~Hz}, 2 \mathrm{H}), 1.84-1.74(\mathrm{~m}, 2 \mathrm{H}), 1.32(\mathrm{t}, J=7.2$ $\mathrm{Hz}, 6 \mathrm{H}), 0.89$ (s, 9H), $0.04(\mathrm{~s}, 6 \mathrm{H}) .{ }^{13} \mathrm{C} \mathrm{NMR}\left(126 \mathrm{MHz}, \mathrm{CDCl}_{3}\right) \delta 130.6\left(\mathrm{~d}, J_{\mathrm{C}-\mathrm{P}}=18.0 \mathrm{~Hz}\right), 127.5$, $62.9,61.3\left(\mathrm{~d}, J_{\mathrm{C}-\mathrm{P}}=6.0 \mathrm{~Hz}, 2 \mathrm{C}\right), 36.0,25.8(3 \mathrm{C}), 25.6\left(\mathrm{~d}, J_{\mathrm{C}-\mathrm{P}}=140 \mathrm{~Hz}\right), 25.4\left(\mathrm{~d}, J_{\mathrm{C}-\mathrm{P}}=4.8 \mathrm{~Hz}\right)$, $18.2,16.3\left(\mathrm{~d}, J_{\mathrm{C}-\mathrm{P}}=6.0 \mathrm{~Hz}, 2 \mathrm{C}\right),-5.4(2 \mathrm{C}) .{ }^{31} \mathrm{P} \mathrm{NMR}\left(202 \mathrm{MHz}, \mathrm{CDCl}_{3}\right) \delta 32.3$. IR (ATR) v 2929, 1248, 1028, 833, $774 \mathrm{~cm}^{-1}$. HRMS (FD) $\mathrm{m} / z$ : $[\mathrm{M}+\mathrm{H}]^{+}$calcd for $\mathrm{C}_{16} \mathrm{H}_{36} \mathrm{O}_{4} \mathrm{PSi}$ 351.2121; found: 351.2120 .

\section{General Procedure for Preparations of Cyclization Precursor ${ }^{[10]}$}

\section{Procedure B}

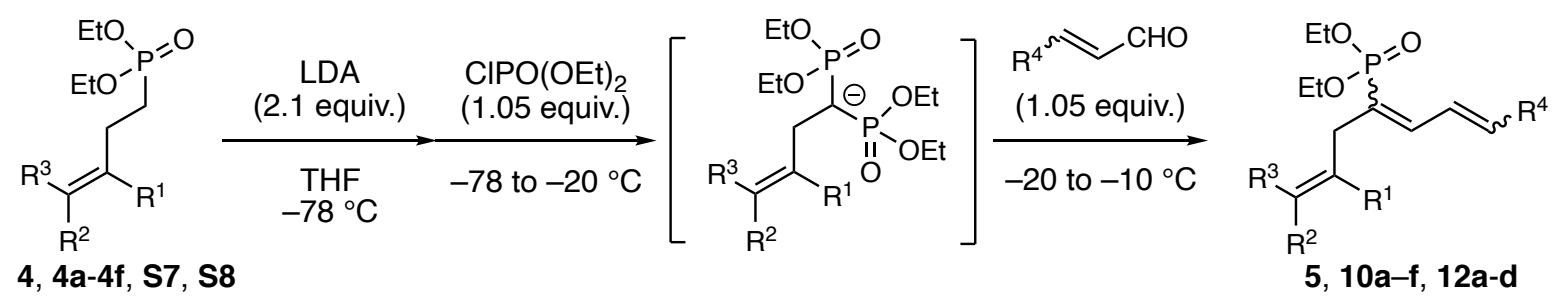

To a solution of diisopropylamine (2.10 equiv.) in THF at $0{ }^{\circ} \mathrm{C}$ was slowly added a solution of $n$ BuLi in $n$-hexane (2.10 equiv.). After stirring for $20 \sim 30 \mathrm{~min}$, the mixture was cooled to $-78{ }^{\circ} \mathrm{C}$, 
and a solution of $\gamma, \delta$-unsaturated phosphonate in THF was added via cannula (at this time $0.2 \mathrm{M}$ to $\gamma, \delta$-unsaturated phosphonate). After stirring for $15 \mathrm{~min}$ at $-78{ }^{\circ} \mathrm{C}$, diethyl chlorophosphate (1.05 equiv.) was added dropwise. The mixture was warmed to $-20{ }^{\circ} \mathrm{C}$ over ca. $0.5 \mathrm{~h}$, and then $\alpha, \beta-$ unsaturated aldehyde ( 1.05 equiv.) was added. When the bath temperature reached $-10{ }^{\circ} \mathrm{C}$ under ambient atmosphere, the reaction was quenched with saturated aqueous $\mathrm{NH}_{4} \mathrm{Cl}$. After EtOAc was added, the two layers were separated, and the aqueous layer was extracted with EtOAc (2 times). The combined organic layers were dried over $\mathrm{MgSO}_{4}$, filtered, and concentrated under reduced pressure. Purification by silica gel flash column chromatography afforded cyclization precursor.

\section{Synthesis of Cyclization Precursors}

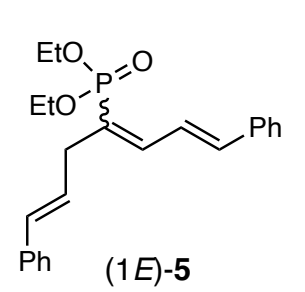
hexane/EtOAc $=4: 1$ to $1: 1)$ afforded $(1 E, 3 E)-5(961 \mathrm{mg}, 2.51 \mathrm{mmol}, 50 \%)$ and $(1 E, 3 Z)-5(828 \mathrm{mg}$, $2.17 \mathrm{mmol}, 43 \%)$.

(1E,3E)-5: pale orange oil. ${ }^{1} \mathrm{H}-\mathrm{NMR}\left(500 \mathrm{MHz}, \mathrm{CDCl}_{3}\right) \delta 7.46(\mathrm{~d}, J=7.5 \mathrm{~Hz}, 2 \mathrm{H}), 7.37-7.23(\mathrm{~m}$, $8 \mathrm{H}), 7.20$ (t, $J=7.2 \mathrm{~Hz}, 1 \mathrm{H}), 7.10(\mathrm{ddd}, J=15.5,11.5,2.3 \mathrm{~Hz}, 1 \mathrm{H}), 6.88$ (d, $J=15.5 \mathrm{~Hz}, 1 \mathrm{H}), 6.49$ $(\mathrm{d}, J=15.5 \mathrm{~Hz}, 1 \mathrm{H}), 6.22(\mathrm{dt}, J=15.5,6.6 \mathrm{~Hz}, 1 \mathrm{H}), 4.16-4.00(\mathrm{~m}, 4 \mathrm{H}), 3.34(\mathrm{dd}, J=18.3,6.9 \mathrm{~Hz}$, $2 \mathrm{H}), 1.31(\mathrm{t}, J=6.9 \mathrm{~Hz}, 6 \mathrm{H}) .{ }^{13} \mathrm{C} \mathrm{NMR}\left(126 \mathrm{MHz}, \mathrm{CDCl}_{3}\right) \delta 143.8\left(\mathrm{~d}, J_{\mathrm{C}-\mathrm{P}}=12.0 \mathrm{~Hz}\right) 139.9,137.2$, 136.2, 131.2, 128.9, 128.7, 128.5 (2C), $127.2(2 \mathrm{C}), 127.1(2 \mathrm{C}), 126.9\left(\mathrm{~d}, J_{\mathrm{C}-\mathrm{P}}=2.4 \mathrm{~Hz}\right), 126.3(\mathrm{~d}$, $\left.J_{\mathrm{C}-\mathrm{P}}=181 \mathrm{~Hz}\right), 126.0(2 \mathrm{C}), 122.6\left(\mathrm{~d}, J_{\mathrm{C}-\mathrm{P}}=22.8 \mathrm{~Hz}\right), 61.7\left(\mathrm{~d}, J_{\mathrm{C}-\mathrm{P}}=4.8 \mathrm{~Hz}, 2 \mathrm{C}\right), 30.9\left(\mathrm{~d}, J_{\mathrm{C}-\mathrm{P}}=9.6\right.$ $\mathrm{Hz}), 16.3\left(\mathrm{~d}, J_{\mathrm{C}-\mathrm{P}}=6.0 \mathrm{~Hz}, 2 \mathrm{C}\right) .{ }^{31} \mathrm{P} \mathrm{NMR}\left(202 \mathrm{MHz}, \mathrm{CDCl}_{3}\right) \delta 22.2$. IR (ATR) v 2980, 1240, 1019, $961,748 \mathrm{~cm}^{-1}$. HRMS (FD) $\mathrm{m} / z$ : $[\mathrm{M}]^{+}$calcd for $\mathrm{C}_{23} \mathrm{H}_{27} \mathrm{O}_{3} \mathrm{P} 382.1698$, found; 382.1715 .

(1E,3Z)-5: yellow oil. ${ }^{1} \mathrm{H}$ NMR $\left(500 \mathrm{MHz}, \mathrm{CDCl}_{3}\right) \delta 7.92(\mathrm{ddd}, J=15.5,11.5,1.2 \mathrm{~Hz}, 1 \mathrm{H}), 7.49(\mathrm{~d}$, $J=7.5 \mathrm{~Hz}, 2 \mathrm{H}), 7.40(\mathrm{dd}, J=8.6,1.7 \mathrm{~Hz}, 2 \mathrm{H}), 7.36-7.31(\mathrm{~m}, 4 \mathrm{H}), 7.29-7.23(\mathrm{~m}, 2 \mathrm{H}), 6.87(\mathrm{dd}, J$ $=47.0,10.3 \mathrm{~Hz}, 1 \mathrm{H}), 6.69(\mathrm{~d}, J=15.5 \mathrm{~Hz}, 1 \mathrm{H}), 6.50(\mathrm{~d}, J=15.5 \mathrm{~Hz}, 1 \mathrm{H}), 6.27(\mathrm{dt}, J=16.1,7.2 \mathrm{~Hz}$, $1 \mathrm{H}), 4.21-4.06(\mathrm{~m}, 4 \mathrm{H}), 3.24(\mathrm{dd}, J=12.6,6.9 \mathrm{~Hz}, 2 \mathrm{H}), 1.36(\mathrm{t}, J=7.2 \mathrm{~Hz}, 6 \mathrm{H}) .{ }^{13} \mathrm{C}$ NMR $(126$ $\left.\mathrm{MHz} \mathrm{CDCl}_{3}\right) \delta 145.3\left(\mathrm{~d}, J_{\mathrm{C}-\mathrm{P}}=10.8 \mathrm{~Hz}\right), 138.5\left(\mathrm{~d}, J_{\mathrm{C}-\mathrm{P}}=2.4 \mathrm{~Hz}\right), 137.2,136.6,132.2,128.6(2 \mathrm{C})$, 128.5, 128.4, $127.3(2 \mathrm{C}), 127.20,127.17(2 \mathrm{C}), 127.0\left(\mathrm{~d}, J_{\mathrm{C}-\mathrm{P}}=173 \mathrm{~Hz}\right), 126.1(2 \mathrm{C}), 125.7\left(\mathrm{~d}, J_{\mathrm{C}-\mathrm{P}}\right.$ $=8.4 \mathrm{~Hz}), 61.6\left(\mathrm{~d}, J_{\mathrm{C}-\mathrm{P}}=6.0 \mathrm{~Hz}, 2 \mathrm{C}\right), 37.9\left(\mathrm{~d}, J_{\mathrm{C}-\mathrm{P}}=12.0 \mathrm{~Hz}\right), 16.4\left(\mathrm{~d}, J_{\mathrm{C}-\mathrm{P}}=6.0 \mathrm{~Hz}, 2 \mathrm{C}\right) .{ }^{31} \mathrm{P} \mathrm{NMR}$ $\left(202 \mathrm{MHz}, \mathrm{CDCl}_{3}\right) \delta 19.1$. IR (ATR) $v 2981,1235,1047,1017,963,749 \mathrm{~cm}^{-1}$. HRMS (FD) $m / z$ : $[\mathrm{M}]^{+}$calcd for $\mathrm{C}_{23} \mathrm{H}_{27} \mathrm{O}_{3} \mathrm{P} 382.1698$; found: 382.1682 . 


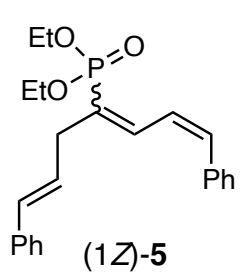

According to Procedure $\mathrm{B}$, purification by flash column chromatography $\left(\mathrm{SiO}_{2}, n\right.$ hexane/EtOAc $=2: 1)$ afforded an isomeric mixture of phosphonate $(1 Z)-5$ (326 mg, $0.852 \mathrm{mmol}, 85 \%, 3 E / 3 Z=1.0: 1.4)$ by using $4(268 \mathrm{mg}, 1.00 \mathrm{mmol})$, diisopropylamine $(296 \mu \mathrm{L}, 2.10 \mathrm{mmol})$, a $2.64 \mathrm{M}$ solution of $n$-BuLi in $n$-hexane (795 $\mu \mathrm{L}, 2.10 \mathrm{mmol})$, diethyl chlorophosphate $(151 \mu \mathrm{L}, 1.05 \mathrm{mmol})$ and ciscinnamaldehyde $(133 \mu \mathrm{L}, 1.05 \mathrm{mmol})$.

(1Z,3E)-5 and (1Z,3Z)-5 were partially separated by flash column chromatography $\left(\mathrm{SiO}_{2}, n\right.$ hexane/EtOAc $=2: 1)$ to give analytically pure samples.

(1Z,3E)-5: pale yellow oil. ${ }^{1} \mathrm{H}$ NMR $\left(500 \mathrm{MHz}, \mathrm{CDCl}_{3}\right) \delta 7.54(\mathrm{dd}, J=22.9,12.0 \mathrm{~Hz}, 1 \mathrm{H}), 7.40-$ $7.32(\mathrm{~m}, 6 \mathrm{H}), 7.32-7.18(\mathrm{~m}, 3 \mathrm{H}), 7.21(\mathrm{t}, J=7.5 \mathrm{~Hz}, 1 \mathrm{H}), 6.81(\mathrm{dd}, J=11.5,3.5 \mathrm{~Hz}, 1 \mathrm{H}), 6.57$ (ddd, $J=12.0,11.5,3.5 \mathrm{~Hz}, 1 \mathrm{H}), 6.48(\mathrm{~d}, J=15.5 \mathrm{~Hz}, 1 \mathrm{H}), 6.22(\mathrm{dt}, J=15.5,6.3 \mathrm{~Hz}, 1 \mathrm{H}), 4.13-4.00$ (m, 4H), $3.34(\mathrm{dd}, J=18.4,6.3 \mathrm{~Hz}, 2 \mathrm{H}), 1.28(\mathrm{t}, J=6.9 \mathrm{~Hz}, 6 \mathrm{H}) .{ }^{13} \mathrm{C} \mathrm{NMR}\left(126 \mathrm{MHz}, \mathrm{CDCl}_{3}\right) \delta 138.9$ $\left(\mathrm{d}, J_{\mathrm{C}-\mathrm{P}}=12.0 \mathrm{~Hz}\right), 137.3,136.8,136.2,131.2,129.3(2 \mathrm{C}), 128.7\left(\mathrm{~d}, J_{\mathrm{C}-\mathrm{P}}=181 \mathrm{~Hz}\right), 128.5(2 \mathrm{C})$, $128.4,128.0,127.2(2 \mathrm{C}), 126.6\left(\mathrm{~d}, J_{\mathrm{C}-\mathrm{P}}=2.4 \mathrm{~Hz}\right), 126.1(2 \mathrm{C}), 123.6\left(\mathrm{~d}, J_{\mathrm{C}-\mathrm{P}}=22.8 \mathrm{~Hz}\right), 61.7\left(\mathrm{~d}, J_{\mathrm{C}-}\right.$ $\mathrm{P}=6.0 \mathrm{~Hz}, 2 \mathrm{C}), 30.8\left(\mathrm{~d}, J_{\mathrm{C}-\mathrm{P}}=10.8 \mathrm{~Hz}\right), 16.3\left(\mathrm{~d}, J_{\mathrm{C}-\mathrm{P}}=7.2 \mathrm{~Hz}, 2 \mathrm{C}\right) .{ }^{31} \mathrm{P} \mathrm{NMR}\left(202 \mathrm{MHz}, \mathrm{CDCl}_{3}\right) \delta$ 21.7. IR (ATR) v 2980, 1241, 1020, 961, 745, $695 \mathrm{~cm}^{-1}$. HRMS (FD) $m / z:[\mathrm{M}]^{+}$calcd for $\mathrm{C}_{23} \mathrm{H}_{27} \mathrm{O}_{3} \mathrm{P}$ 382.1698; found: 382.1699 .

(1Z,3Z)-5: pale yellow oil. ${ }^{1} \mathrm{H}$ NMR (500 MHz, $\left.\mathrm{CDCl}_{3}\right) \delta$ 7.34-7.28 (m, 4H), 7.28-7.15 (m, 8H), $6.74(\mathrm{~d}, J=11.5 \mathrm{~Hz}, 1 \mathrm{H}), 6.43(\mathrm{~d}, J=15.5 \mathrm{~Hz}, 1 \mathrm{H}), 6.19(\mathrm{dt}, J=15.5,7.2 \mathrm{~Hz}, 1 \mathrm{H}), 4.19-4.05(\mathrm{~m}$, $4 \mathrm{H}), 3.19$ (dd, $J=12.6,6.9 \mathrm{~Hz}, 2 \mathrm{H}), 1.34(\mathrm{t}, J=7.2 \mathrm{~Hz}, 6 \mathrm{H}) .{ }^{13} \mathrm{C} \mathrm{NMR}\left(126 \mathrm{MHz}, \mathrm{CDCl}_{3}\right) \delta 140.9$ $\left(\mathrm{d}, J_{\mathrm{C}-\mathrm{P}}=10.8 \mathrm{~Hz}\right), 137.2,136.4,135.2,132.2,129.3(2 \mathrm{C}), 129.1\left(\mathrm{~d}, J_{\mathrm{C}-\mathrm{P}}=173 \mathrm{~Hz}\right), 128.4,128.2$ (2C), 127.6, $127.2(2 \mathrm{C}), 127.1(2 \mathrm{C}), 126.2\left(\mathrm{~d}, J_{\mathrm{C}-\mathrm{P}}=8.4 \mathrm{~Hz}\right), 126.1,61.6\left(\mathrm{~d}, J_{\mathrm{C}-\mathrm{P}}=6.0 \mathrm{~Hz}, 2 \mathrm{C}\right), 38.0$ $\left(\mathrm{d}, J_{\mathrm{C}-\mathrm{P}}=12.0 \mathrm{~Hz}\right), 16.3\left(\mathrm{~d}, J_{\mathrm{C}-\mathrm{P}}=7.2 \mathrm{~Hz}, 2 \mathrm{C}\right) .{ }^{31} \mathrm{P} \mathrm{NMR}\left(202 \mathrm{MHz}, \mathrm{CDCl}_{3}\right) \delta 18.7$. IR (ATR) v 2980, 1234, 1019, 958, 745, $694 \mathrm{~cm}^{-1}$. HRMS (FD) calcd for $\mathrm{C}_{23} \mathrm{H}_{27} \mathrm{O}_{3} \mathrm{P}[\mathrm{M}]^{+}:$: 382.1698, found: 382.1695 .

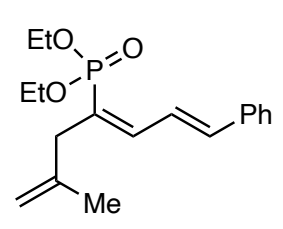

$(3 Z)-10 a$

According to Procedure $\mathrm{B}$, an isomeric mixture of crude phosphonate 10a $(3 E / 3 Z$ $=1: 7)$ was obtained from 4a $(206 \mathrm{mg}, 1.00 \mathrm{mmol})$, diisopropylamine $(296 \mu \mathrm{L}$, $2.10 \mathrm{mmol})$, a $2.67 \mathrm{M}$ solution of $n$-BuLi in $n$-hexane $(0.79 \mathrm{~mL}, 2.10 \mathrm{mmol})$, diethyl chlorophosphate $(151 \mu \mathrm{L}, 1.05 \mathrm{mmol})$ and trans-cinnamaldehyde $(132 \mu \mathrm{L}$, 1.05 mmol). Purification by flash column chromatography $\left(\mathrm{SiO}_{2}, n-\right.$ hexane/EtOAc $=4: 1$ to 2:1) afforded $(3 Z)-10 a(211 \mathrm{mg}, 0.659 \mathrm{mmol}, 66 \%)$.

(3Z)-10a: yellow oil. ${ }^{1} \mathrm{H}$ NMR $\left(500 \mathrm{MHz}, \mathrm{CDCl}_{3}\right) \delta 7.89(\mathrm{dd}, J=15.5,11.5 \mathrm{~Hz}, 1 \mathrm{H}), 7.48(\mathrm{~d}, J=$ $7.4 \mathrm{~Hz}, 2 \mathrm{H}), 7.32(\mathrm{dd}, J=7.4,7.2 \mathrm{~Hz}, 2 \mathrm{H}), 7.28-7.23(\mathrm{~m}, 1 \mathrm{H}), 6.79(\mathrm{dd}, J=48.1,11.5 \mathrm{~Hz}, 1 \mathrm{H})$, $6.68(\mathrm{~d}, J=15.5 \mathrm{~Hz}, 1 \mathrm{H}), 4.91(\mathrm{~s}, 1 \mathrm{H}), 4.83(\mathrm{~s}, 1 \mathrm{H}), 4.17-4.02(\mathrm{~m}, 4 \mathrm{H}), 3.01(\mathrm{~d}, J=12.6 \mathrm{~Hz}, 2 \mathrm{H})$, 
$1.74(\mathrm{~s}, 3 \mathrm{H}), 1.33(\mathrm{t}, J=6.9 \mathrm{~Hz}, 6 \mathrm{H}) .{ }^{13} \mathrm{C} \mathrm{NMR}\left(126 \mathrm{MHz}, \mathrm{CDCl}_{3}\right) \delta 145.4\left(\mathrm{~d}, J_{\mathrm{C}-\mathrm{P}}=10.8 \mathrm{~Hz}\right), 142.9$ $\left(\mathrm{d}, J_{\mathrm{C}-\mathrm{P}}=4.8 \mathrm{~Hz}\right), 138.3\left(\mathrm{~d}, J_{\mathrm{C}-\mathrm{P}}=2.4 \mathrm{~Hz}\right), 136.6,128.6(2 \mathrm{C}), 128.4,127.1(2 \mathrm{C}), 126.2\left(\mathrm{~d}, J_{\mathrm{C}-\mathrm{P}}=\right.$ $174 \mathrm{~Hz}), 125.8\left(\mathrm{~d}, J_{\mathrm{C}-\mathrm{P}}=8.4 \mathrm{~Hz}\right), 113.4,61.5\left(\mathrm{~d}, J_{\mathrm{C}-\mathrm{P}}=4.8 \mathrm{~Hz}, 2 \mathrm{C}\right), 42.3\left(\mathrm{~d}, J_{\mathrm{C}-\mathrm{P}}=10.8 \mathrm{~Hz}\right), 22.1$, $16.3\left(\mathrm{~d}, J_{\mathrm{C}-\mathrm{P}}=6.0 \mathrm{~Hz}, 2 \mathrm{C}\right) .{ }^{31} \mathrm{P} \mathrm{NMR}\left(202 \mathrm{MHz}, \mathrm{CDCl}_{3}\right) \delta$ 19.4. IR (ATR) v 2980, 1235, 1019, 957, $750 \mathrm{~cm}^{-1}$. HRMS (FD) $m / z$ : [M] $]^{+}$calcd for $\mathrm{C}_{18} \mathrm{H}_{25} \mathrm{O}_{3} \mathrm{P} 320.1541$; found: 320.1539 .

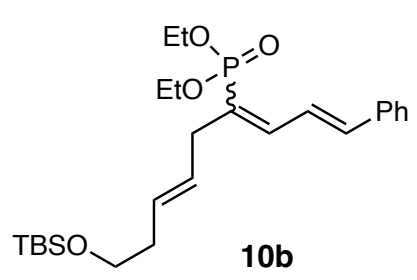

According to Procudure B, an isomeric mixture of crude phosphonate 10b $(3 E / 3 Z=1.0: 1.0)$ was obtained from $4 \mathbf{b}(185 \mathrm{mg}, 0.528 \mathrm{mmol})$, diisopropylamine $(157 \mu \mathrm{L}, 1.11 \mathrm{mmol})$, a $2.67 \mathrm{M}$ solution of $n$-BuLi in $n$-hexane $(416 \mu \mathrm{L}, 1.11 \mathrm{mmol})$, diethyl chlorophosphate $(79.7 \mu \mathrm{L}, 0.554$ mmol) and trans-cinnamaldehyde $(69.7 \mu \mathrm{L}, 0.554 \mathrm{mmol})$. Purification by flash column chromatography $\left(\mathrm{SiO}_{2}, n\right.$-hexane/EtOAc $=4: 1$ to $\left.1: 1\right)$ afforded $(3 E)-\mathbf{1 0 b}(88.7 \mathrm{mg}$, $0.191 \mathrm{mmol}, 36 \%)$ and (3Z)-10b (87.7 $\mathrm{mg}, 0.189 \mathrm{mmol}, 36 \%)$.

(3E)-10b: yellow oil. ${ }^{1} \mathrm{H}$ NMR $\left(500 \mathrm{MHz}, \mathrm{CDCl}_{3}\right) \delta 7.45(\mathrm{~d}, J=7.4 \mathrm{~Hz}, 2 \mathrm{H}), 7.35(\mathrm{dd}, J=7.4,7.4$ $\mathrm{Hz}, 2 \mathrm{H}), 7.30(\mathrm{t}, J=7.4 \mathrm{~Hz}, 1 \mathrm{H}), 7.19(\mathrm{dd}, J=21.8,10.9 \mathrm{~Hz}, 1 \mathrm{H}), 7.04(\mathrm{ddd}, J=15.5,10.9,1.7 \mathrm{~Hz}$, $1 \mathrm{H}), 6.83(\mathrm{~d}, J=15.5 \mathrm{~Hz}, 1 \mathrm{H}), 5.60-5.46(\mathrm{~m}, 2 \mathrm{H}), 4.15-3.98(\mathrm{~m}, 4 \mathrm{H}), 3.60(\mathrm{t}, J=6.9 \mathrm{~Hz}, 2 \mathrm{H}), 3.13$ $(\mathrm{dd}, J=20.2,5.8 \mathrm{~Hz}, 2 \mathrm{H}), 2.23(\mathrm{dt}, J=6.9,6.9 \mathrm{~Hz}, 2 \mathrm{H}), 1.32(\mathrm{t}, J=7.2 \mathrm{~Hz}, 6 \mathrm{H}), 0.87(\mathrm{~s}, 9 \mathrm{H}), 0.02$ $(\mathrm{s}, 6 \mathrm{H}) .{ }^{13} \mathrm{C} \mathrm{NMR}\left(126 \mathrm{MHz}, \mathrm{CDCl}_{3}\right) \delta 143.3\left(\mathrm{~d}, J_{\mathrm{C}-\mathrm{P}}=12.0 \mathrm{~Hz}\right), 139.4,136.4,128.74,128.71(2 \mathrm{C})$, $128.68\left(\mathrm{~d}, J_{\mathrm{C}-\mathrm{P}}=2.4 \mathrm{~Hz}\right), 128.2,127.1(2 \mathrm{C}), 126.9\left(\mathrm{~d}, J_{\mathrm{C}-\mathrm{P}}=181 \mathrm{~Hz}\right), 122.9\left(\mathrm{~d}, J_{\mathrm{C}-\mathrm{P}}=22.8 \mathrm{~Hz}\right), 63.0$, $61.6\left(\mathrm{~d}, J_{\mathrm{C}-\mathrm{P}}=4.8 \mathrm{~Hz}, 2 \mathrm{C}\right), 36.1,30.7\left(\mathrm{~d}, J_{\mathrm{C}-\mathrm{P}}=9.6 \mathrm{~Hz}\right), 25.9(3 \mathrm{C}), 18.3,16.3\left(\mathrm{~d}, J_{\mathrm{C}-\mathrm{P}}=6.0 \mathrm{~Hz}, 2 \mathrm{C}\right)$, $-5.3(2 \mathrm{C}) .{ }^{31} \mathrm{P}$ NMR $\left(202 \mathrm{MHz}, \mathrm{CDCl}_{3}\right) \delta 22.2$. IR (ATR) $v 2928,1247,1023,963,834,774 \mathrm{~cm}^{-1}$. HRMS (FD) $m / z$ : [M] $]^{+}$calcd for $\mathrm{C}_{25} \mathrm{H}_{41} \mathrm{O}_{4} \mathrm{PSi} 464.2512$; found: 464.2514.

(3Z)-10b: yellow oil. ${ }^{1} \mathrm{H} \mathrm{NMR}\left(500 \mathrm{MHz}, \mathrm{CDCl}_{3}\right) \delta 7.88(\mathrm{dd}, J=15.5,10.9 \mathrm{~Hz}, 1 \mathrm{H}), 7.47(\mathrm{~d}, J=$ $7.5 \mathrm{~Hz}, 2 \mathrm{H}), 7.32(\mathrm{dd}, J=7.5,7.2 \mathrm{~Hz}, 2 \mathrm{H}), 7.28-7.23(\mathrm{~m}, 1 \mathrm{H}), 6.79$ (dd, $J=48.7,10.9 \mathrm{~Hz}, 1 \mathrm{H})$, $6.66(\mathrm{~d}, J=15.5 \mathrm{~Hz}, 1 \mathrm{H}), 5.55-5.52(\mathrm{~m}, 2 \mathrm{H}), 4.17-2.02(\mathrm{~m}, 4 \mathrm{H}), 3.65(\mathrm{t}, J=6.9 \mathrm{~Hz}, 2 \mathrm{H}), 3.01(\mathrm{dd}$, $J=12.1,3.5 \mathrm{~Hz}, 2 \mathrm{H}), 2.28(\mathrm{dt}, J=6.9,6.9 \mathrm{~Hz}, 2 \mathrm{H}), 1.33(\mathrm{t}, J=6.9 \mathrm{~Hz}, 6 \mathrm{H}), 0.90(\mathrm{~s}, 9 \mathrm{H}), 0.06$ (s, $6 \mathrm{H}) ;{ }^{13} \mathrm{C} \mathrm{NMR}\left(126 \mathrm{MHz}, \mathrm{CDCl}_{3}\right) \delta 144.8\left(\mathrm{~d}, J_{\mathrm{C}-\mathrm{P}}=12.0 \mathrm{~Hz}\right), 138.1\left(\mathrm{~d}, J_{\mathrm{C}-\mathrm{P}}=2.4 \mathrm{~Hz}\right), 136.7,129.5$, $128.9\left(\mathrm{~d}, J_{\mathrm{C}-\mathrm{P}}=6.0 \mathrm{~Hz}\right), 128.6(2 \mathrm{C}), 128.3,127.6\left(\mathrm{~d}, J_{\mathrm{C}-\mathrm{P}}=173 \mathrm{~Hz}\right), 127.1(2 \mathrm{C}), 125.8\left(\mathrm{~d}, J_{\mathrm{C}-\mathrm{P}}=8.4\right.$ $\mathrm{Hz}), 63.1,61.4\left(\mathrm{~d}, J_{\mathrm{C}-\mathrm{P}}=6.0 \mathrm{~Hz}, 2 \mathrm{C}\right), 37.5\left(\mathrm{~d}, J_{\mathrm{C}-\mathrm{P}}=10.8 \mathrm{~Hz}\right), 36.2,25.9(3 \mathrm{C}), 18.3,16.3\left(\mathrm{~d}, J_{\mathrm{C}-\mathrm{P}}=\right.$ $6.0 \mathrm{~Hz}, 2 \mathrm{C}$ ), -5.3 (2C). ${ }^{31} \mathrm{P}$ NMR (202 MHz, $\mathrm{CDCl}_{3}$ ) $\delta$ 19.1. IR (ATR) v 2928, 1238, 1022, 834, 774 $\mathrm{cm}^{-1}$. HRMS (FD) $\mathrm{m} / z$ : [M] $]^{+}$calcd for $\mathrm{C}_{25} \mathrm{H}_{41} \mathrm{O}_{3} \mathrm{PSi} 464.2512$; found: 464.2526 .

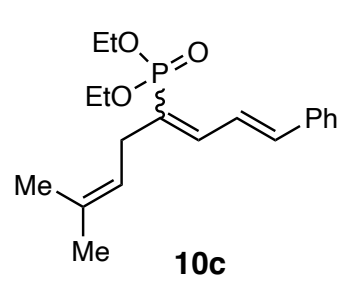

According to Procedure B, an isomeric mixture of crude phosphonate 10c $(3 E / 3 Z=1.4: 1.0)$ was obtained from $4 \mathbf{c}(220 \mathrm{mg}, 1.00 \mathrm{mmol})$, diisopropylamine $(296 \mu \mathrm{L}, 2.10 \mathrm{mmol})$, a $2.64 \mathrm{M}$ solution of $n$-BuLi in $n$ hexane $(795 \mu \mathrm{L}, 2.10 \mathrm{mmol})$, diethyl chlorophosphate $(151 \mu \mathrm{L}, 1.05 \mathrm{mmol})$ and trans-cinnamaldehyde $(132 \mu \mathrm{L}, 1.05 \mathrm{mmol})$. Purification by flash column 
chromatography $\left(\mathrm{SiO}_{2}, n\right.$-hexane/EtOAc $=4: 1$ to $\left.1: 1\right)$ afforded $(3 E)-10 \mathrm{c}(152 \mathrm{mg}, 0.455 \mathrm{mmol}$, $46 \%$ ) and (3Z)-10c (104 mg, $0.311 \mathrm{mmol}, 31 \%)$.

(3E)-10c: yellow oil. ${ }^{1} \mathrm{H}$ NMR $\left(500 \mathrm{MHz}, \mathrm{CDCl}_{3}\right) \delta 7.45(\mathrm{~d}, J=7.5 \mathrm{~Hz}, 2 \mathrm{H}), 7.35(\mathrm{dd}, J=7.5,7.2$ $\mathrm{Hz}, 2 \mathrm{H}), 7.29$ (t, $J=7.2 \mathrm{~Hz}, 1 \mathrm{H}), 7.16(\mathrm{dd}, J=21.8,10.9 \mathrm{~Hz}, 1 \mathrm{H}), 7.04(\mathrm{ddd}, J=15.5,10.9,2.3 \mathrm{~Hz}$, $1 \mathrm{H}), 6.83$ (d, $J=15.5 \mathrm{~Hz}, 1 \mathrm{H}), 5.10$ (br t, $J=6.9 \mathrm{~Hz}, 1 \mathrm{H}), 4.14-3.98$ (m, 4H), 3.12 (dd, $J=18.4$, $7.5 \mathrm{~Hz}, 2 \mathrm{H}), 1.76(\mathrm{~s}, 3 \mathrm{H}), 1.71(\mathrm{~d}, J=1.2 \mathrm{~Hz}, 3 \mathrm{H}), 1.32$ (t, $J=6.9 \mathrm{~Hz}, 6 \mathrm{H}) .{ }^{13} \mathrm{C} \mathrm{NMR}(126 \mathrm{MHz}$, $\left.\mathrm{CDCl}_{3}\right) \delta 142.7\left(\mathrm{~d}, J_{\mathrm{C}-\mathrm{P}}=12.0 \mathrm{~Hz}\right), 139.0,136.4,132.3,128.7(2 \mathrm{C}), 128.6,128.0\left(\mathrm{~d}, J_{\mathrm{C}-\mathrm{P}}=179 \mathrm{~Hz}\right)$, $127.0(2 \mathrm{C}), 122.9\left(\mathrm{~d}, J_{\mathrm{C}-\mathrm{P}}=24.0 \mathrm{~Hz}\right), 121.7\left(\mathrm{~d}, J_{\mathrm{C}-\mathrm{P}}=4.4 \mathrm{~Hz}\right), 61.5\left(\mathrm{~d}, J_{\mathrm{C}-\mathrm{P}}=4.8 \mathrm{~Hz}, 2 \mathrm{C}\right), 26.6(\mathrm{~d}$, $\left.J_{\mathrm{C}-\mathrm{P}}=9.6 \mathrm{~Hz}\right), 25.6,17.9,16.2\left(\mathrm{~d}, J_{\mathrm{C}-\mathrm{P}}=6.0 \mathrm{~Hz}, 2 \mathrm{C}\right) .{ }^{31} \mathrm{P} \mathrm{NMR}\left(202 \mathrm{MHz}, \mathrm{CDCl}_{3}\right) \delta 22.5$. IR (ATR) $v$ 2979, 1243, 1021, 957, $750 \mathrm{~cm}^{-1}$. HRMS (FD) $m / z$ : [M] $]^{+}$calcd for $\mathrm{C}_{19} \mathrm{H}_{27} \mathrm{O}_{3} \mathrm{P}$ 334.1698; found: 334.1681.

(3Z)-10c: yellow oil. ${ }^{1} \mathrm{H} \mathrm{NMR}\left(500 \mathrm{MHz}, \mathrm{CDCl}_{3}\right) \delta 7.90(\mathrm{dd}, J=15.5,11.5 \mathrm{~Hz}, 1 \mathrm{H}), 7.47(\mathrm{~d}, J=$ $7.5 \mathrm{~Hz}, 2 \mathrm{H}), 7.32$ (dd, $J=7.5,7.4 \mathrm{~Hz}, 2 \mathrm{H}), 7.25(\mathrm{t}, J=7.4 \mathrm{~Hz}, 1 \mathrm{H}), 6.77(\mathrm{dd}, J=48.7,11.5 \mathrm{~Hz}, 1 \mathrm{H})$, $6.65(\mathrm{~d}, J=15.5 \mathrm{~Hz}, 1 \mathrm{H}), 5.20(\mathrm{t}, J=7.2 \mathrm{~Hz}, 1 \mathrm{H}), 4.16-4.02(\mathrm{~m}, 4 \mathrm{H}), 3.00(\mathrm{dd}, J=10.9,7.5 \mathrm{~Hz}$, 2H), 1.77 (s, 3H), $1.66(\mathrm{~s}, 3 \mathrm{H}), 1.34$ (t, $J=7.2 \mathrm{~Hz}, 6 \mathrm{H}) .{ }^{13} \mathrm{C} \mathrm{NMR}\left(126 \mathrm{MHz}, \mathrm{CDCl}_{3}\right) \delta 144.0(\mathrm{~d}$, $\left.J_{\mathrm{C}-\mathrm{P}}=12.0 \mathrm{~Hz}\right), 137.8\left(\mathrm{~d}, J_{\mathrm{C}-\mathrm{P}}=2.4 \mathrm{~Hz}\right), 136.7,134.1,128.5(2 \mathrm{C}), 128.2,127.0(2 \mathrm{C}), 126.5\left(\mathrm{~d}, J_{\mathrm{C}-\mathrm{P}}\right.$ $=169 \mathrm{~Hz}), 125.8,120.9\left(\mathrm{~d}, J_{\mathrm{C}-\mathrm{P}}=7.2 \mathrm{~Hz}\right), 61.3\left(\mathrm{~d}, J_{\mathrm{C}-\mathrm{P}}=4.8 \mathrm{~Hz}, 2 \mathrm{C}\right), 32.8\left(\mathrm{~d}, J_{\mathrm{C}-\mathrm{P}}=10.8 \mathrm{~Hz}\right), 25.7$, $17.7,16.3\left(\mathrm{~d}, J_{\mathrm{C}-\mathrm{P}}=6.0 \mathrm{~Hz}, 2 \mathrm{C}\right) .{ }^{31} \mathrm{P} \mathrm{NMR}\left(202 \mathrm{MHz}, \mathrm{CDCl}_{3}\right) \delta 19.5$. IR (ATR) v 2980, 1237, 1022, $959,751 \mathrm{~cm}^{-1}$. HRMS (FD) $m / z$ : [M] $]^{+}$calcd for $\mathrm{C}_{19} \mathrm{H}_{27} \mathrm{O}_{3} \mathrm{P} 334.1698$; found: 334.1699.

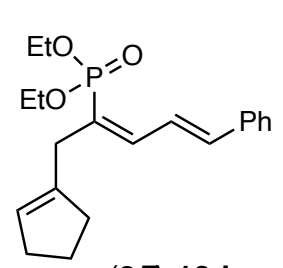

$(3 Z)-10 d$

According to Procedure B, an isomeric mixture of crude phosphonate 10d ( $3 E / 3 Z$ $=1: 6)$ was obtained from $4 \mathbf{d}(103 \mathrm{mg}, 0.443 \mathrm{mmol})$, diisopropylamine $(131 \mu \mathrm{L}$, $0.930 \mathrm{mmol})$, a $2.67 \mathrm{M}$ solution of $n$-BuLi in $n$-hexane $(348 \mu \mathrm{L}, 0.930 \mathrm{mmol})$, diethyl chlorophosphate $(66.9 \mu \mathrm{L}, 0.465 \mathrm{mmol})$ and trans-cinnamaldehyde $(58.5$ $\mu \mathrm{L}, 0.465 \mathrm{mmol})$. Purification by flash column chromatography $\left(\mathrm{SiO}_{2}, n-\right.$ hexane/EtOAc $=2: 1)$ afforded phosphonate $(3 Z)-10 d(106 \mathrm{mg}, 0.306 \mathrm{mmol}$, $69 \%)$

(3Z)-10d: yellow oil. ${ }^{1} \mathrm{H}$ NMR $\left(500 \mathrm{MHz}, \mathrm{CDCl}_{3}\right) \delta 7.88(\mathrm{dd}, J=15.5,11.5 \mathrm{~Hz}, 1 \mathrm{H}), 7.48(\mathrm{~d}, J=$ $7.4 \mathrm{~Hz}, 2 \mathrm{H}), 7.32(\mathrm{dd}, J=7.4,7.2 \mathrm{~Hz}, 2 \mathrm{H}), 7.25(\mathrm{t}, J=7.2 \mathrm{~Hz}, 1 \mathrm{H}), 6.76(\mathrm{dd}, J=48.7,12.1 \mathrm{~Hz}$, $1 \mathrm{H}), 6.66(\mathrm{~d}, J=16.1 \mathrm{~Hz}, 1 \mathrm{H}), 5.49(\mathrm{~s}, 1 \mathrm{H}), 4.15-4.00(\mathrm{~m}, 4 \mathrm{H}), 3.08(\mathrm{~d}, J=13.2 \mathrm{~Hz}, 2 \mathrm{H}), 2.38-$ $2.30(\mathrm{~m}, 2 \mathrm{H}), 2.25$ (br t, $J=6.6 \mathrm{~Hz}, 2 \mathrm{H}), 1.89$ (tt, $J=7.4,7.4 \mathrm{~Hz}, 2 \mathrm{H}), 1.32(\mathrm{t}, J=7.2 \mathrm{~Hz}, 6 \mathrm{H}) .{ }^{13} \mathrm{C}$ $\operatorname{NMR}\left(126 \mathrm{MHz}, \mathrm{CDCl}_{3}\right) \delta 145.2\left(\mathrm{~d}, J_{\mathrm{C}-\mathrm{P}}=12.0 \mathrm{~Hz}\right), 141.6\left(\mathrm{~d}, J_{\mathrm{C}-\mathrm{P}}=4.8 \mathrm{~Hz}\right), 138.0\left(\mathrm{~d}, J_{\mathrm{C}-\mathrm{P}}=2.4\right.$ $\mathrm{Hz}), 136.7,128.6$ (2C), 128.3, $127.1(2 \mathrm{C}), 127.0,126.5$ (d, $\left.J_{\mathrm{C}-\mathrm{P}}=174 \mathrm{~Hz}\right), 125.8$ (d, $\left.J_{\mathrm{C}-\mathrm{P}}=8.4 \mathrm{~Hz}\right)$, $61.4\left(\mathrm{~d}, J_{\mathrm{C}-\mathrm{P}}=4.8 \mathrm{~Hz}, 2 \mathrm{C}\right), 35.9\left(\mathrm{~d}, J_{\mathrm{C}-\mathrm{P}}=12.0 \mathrm{~Hz}\right), 34.6,32.5,23.5,16.3\left(\mathrm{~d}, J_{\mathrm{C}-\mathrm{P}}=7.2 \mathrm{~Hz}, 2 \mathrm{C}\right) .{ }^{31} \mathrm{P}$ NMR (202 MHz, $\left.\mathrm{CDCl}_{3}\right) \delta 19.5$. IR (ATR) $v 2980,1235,1020,956,750 \mathrm{~cm}^{-1}$. HRMS (FD) $m / z$ : $[\mathrm{M}]^{+}$calcd for $\mathrm{C}_{20} \mathrm{H}_{27} \mathrm{O}_{3} \mathrm{P} 346.1698$; found: 346.1689 . 


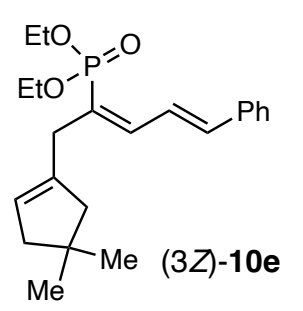

$73 \%)$.

According to Procedure $\mathrm{B}$, an isomeric mixture of crude phosphonate 10e $(3 E / 3 Z$ $=2: 11)$ was obtained from $4 \mathrm{e}(130 \mathrm{mg}, 0.500 \mathrm{mmol})$, diisopropylamine $(148 \mu \mathrm{L}$, $1.05 \mathrm{mmol})$, a $2.67 \mathrm{M}$ solution of $n$-BuLi in $n$-hexane $(393 \mu \mathrm{L}, 1.05 \mathrm{mmol})$, diethyl chlorophosphate $(75.5 \mu \mathrm{L}, 0.525 \mathrm{mmol})$ and trans-cinnamaldehyde $(66.1$ $\mu \mathrm{L}, 0.525 \mathrm{mmol})$. Purification by flash column chromatography $\left(\mathrm{SiO}_{2}, n\right.$ hexane $/$ EtOAc $=4: 1)$ afforded phosphonate $(3 Z)-10 e(136 \mathrm{mg}, 0.363 \mathrm{mmol}$,

(3Z)-10e: yellow oil. ${ }^{1} \mathrm{H}$ NMR $\left(500 \mathrm{MHz}, \mathrm{CDCl}_{3}\right) \delta 7.88(\mathrm{dd}, J=15.5,11.5 \mathrm{~Hz}, 1 \mathrm{H}), 7.48(\mathrm{~d}, J=$ $7.5 \mathrm{~Hz}, 2 \mathrm{H}), 7.32(\mathrm{dd}, J=7.5,7.3 \mathrm{~Hz}, 2 \mathrm{H}), 7.28-7.23(\mathrm{~m}, 1 \mathrm{H}), 6.74(\mathrm{dd}, J=48.1,11.5 \mathrm{~Hz}, 1 \mathrm{H})$, $6.65(\mathrm{~d}, J=15.5 \mathrm{~Hz}, 1 \mathrm{H}), 5.36(\mathrm{br} \mathrm{s}, 1 \mathrm{H}), 4.16-4.00(\mathrm{~m}, 4 \mathrm{H}), 3.03(\mathrm{~d}, J=12.6 \mathrm{~Hz}, 2 \mathrm{H}), 2.16(\mathrm{~d}, J$ $=1.8 \mathrm{~Hz}, 2 \mathrm{H}), 2.07(\mathrm{~s}, 2 \mathrm{H}), 1.33(\mathrm{t}, J=7.2 \mathrm{~Hz}, 6 \mathrm{H}), 1.09(\mathrm{~s}, 6 \mathrm{H}) \cdot{ }^{13} \mathrm{C} \mathrm{NMR}\left(126 \mathrm{MHz}, \mathrm{CDCl}_{3}\right) \delta$ $145.2\left(\mathrm{~d}, J_{\mathrm{C}-\mathrm{P}}=10.8 \mathrm{~Hz}\right), 140.0\left(\mathrm{~d}, J_{\mathrm{C}-\mathrm{P}}=4.8 \mathrm{~Hz}\right), 138.0\left(\mathrm{~d}, J_{\mathrm{C}-\mathrm{P}}=2.4 \mathrm{~Hz}\right), 136.7,128.6(2 \mathrm{C}), 128.3$, $127.1(2 \mathrm{C}), 126.4\left(\mathrm{~d}, J_{\mathrm{C}-\mathrm{P}}=173 \mathrm{~Hz}\right), 125.9\left(\mathrm{~d}, J_{\mathrm{C}-\mathrm{P}}=8.4 \mathrm{~Hz}\right), 125.8,61.4\left(\mathrm{~d}, J_{\mathrm{C}-\mathrm{P}}=4.8 \mathrm{~Hz}, 2 \mathrm{C}\right)$, 49.8, 47.8, 38.6, $36.0\left(\mathrm{~d}, J_{\mathrm{C}-\mathrm{P}}=10.8 \mathrm{~Hz}\right), 30.0(2 \mathrm{C}), 16.4\left(\mathrm{~d}, J_{\mathrm{C}-\mathrm{P}}=7.2 \mathrm{~Hz}, 2 \mathrm{C}\right) .{ }^{31} \mathrm{P}$ NMR $(202 \mathrm{MHz}$, $\left.\mathrm{CDCl}_{3}\right) \delta$ 19.2. IR (ATR) $\vee 2951,1236,1021,957,750 \mathrm{~cm}^{-1}$. HRMS (FD) $\mathrm{m} / z:[\mathrm{M}]^{+}$calcd for $\mathrm{C}_{22} \mathrm{H}_{31} \mathrm{O}_{3} \mathrm{P}$ 374.2011; found: 374.2003 .

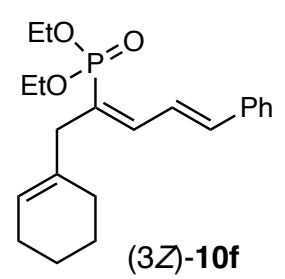

According to Procedure $\mathrm{B}$, an isomeric mixture of crude phosphonate $10 \mathbf{f}(3 E / 3 Z$ $=1: 11)$ was obtained from $4 \mathbf{f}(198 \mathrm{mg}, 0.804 \mathrm{mmol})$, diisopropylamine $(239 \mu \mathrm{L}$, $1.69 \mathrm{mmol})$, a $2.67 \mathrm{M}$ solution of $n$-BuLi in $n$-hexane $(633 \mu \mathrm{L}, 1.69 \mathrm{mmol})$, diethyl chlorophosphate $(121 \mu \mathrm{L}, 0.844 \mathrm{mmol})$ and trans-cinnamaldehyde (109 $\mu \mathrm{L}, 0.844 \mathrm{mmol})$. Purification by flash column chromatography $\left(\mathrm{SiO}_{2}, n-\right.$ hexane/EtOAc $=4: 1)$ afforded phosphonate $(3 Z)-10 f(188 \mathrm{mg}, 0.522 \mathrm{mmol}, 65 \%)$.

(3Z)-10f: pale yellow oil. ${ }^{1} \mathrm{H}$ NMR $\left(500 \mathrm{MHz}, \mathrm{CDCl}_{3}\right) \delta 7.89(\mathrm{dd}, J=15.5,11.5 \mathrm{~Hz}, 1 \mathrm{H}), 7.48(\mathrm{~d}, J$ $=7.4 \mathrm{~Hz}, 2 \mathrm{H}), 7.32(\mathrm{dd}, J=7.5,7.4 \mathrm{~Hz}, 2 \mathrm{H}), 7.25(\mathrm{t}, J=7.5 \mathrm{~Hz}, 1 \mathrm{H}), 6.73(\mathrm{dd}, J=48.1,11.5 \mathrm{~Hz}$, $1 \mathrm{H}), 6.66(\mathrm{~d}, J=15.5 \mathrm{~Hz}, 1 \mathrm{H}), 5.55(\mathrm{br} \mathrm{s}, 1 \mathrm{H}), 4.15-4.00(\mathrm{~m}, 4 \mathrm{H}), 2.93$ (d, $J=13.2 \mathrm{~Hz}, 2 \mathrm{H}), 2.04$ (br s, 2H), 1.92 (br s, 2H), 1.66-1.54 (m, 4H), $1.33(\mathrm{t}, J=7.2 \mathrm{~Hz}, 6 \mathrm{H}) .{ }^{13} \mathrm{C} \mathrm{NMR}\left(126 \mathrm{MHz}, \mathrm{CDCl}_{3}\right)$ $\delta 145.0\left(\mathrm{~d}, J_{\mathrm{C}-\mathrm{P}}=10.8 \mathrm{~Hz}\right), 137.9\left(\mathrm{~d}, J_{\mathrm{C}-\mathrm{P}}=2.4 \mathrm{~Hz}\right), 136.7,134.8\left(\mathrm{~d}, J_{\mathrm{C}-\mathrm{P}}=6.0 \mathrm{~Hz}\right), 128.6(2 \mathrm{C})$, $128.3,127.1(2 \mathrm{C}), 126.6\left(\mathrm{~d}, J_{\mathrm{C}-\mathrm{P}}=173 \mathrm{~Hz}\right), 125.9\left(\mathrm{~d}, J_{\mathrm{C}-\mathrm{P}}=8.4 \mathrm{~Hz}\right), 124.6,61.4\left(\mathrm{~d}, J_{\mathrm{C}-\mathrm{P}}=6.0 \mathrm{~Hz}\right.$, 2C), $42.6\left(\mathrm{~d}, J_{\mathrm{C}-\mathrm{P}}=10.8 \mathrm{~Hz}\right), 27.9,25.4,22.9,22.3,16.3$ (d, $\left.J_{\mathrm{C}-\mathrm{P}}=7.2 \mathrm{~Hz}, 2 \mathrm{C}\right) .{ }^{31} \mathrm{P}$ NMR $(202 \mathrm{MHz}$, $\left.\mathrm{CDCl}_{3}\right) \delta$ 19.7. IR (ATR) $\vee 2925,1235,1020,955,749 \mathrm{~cm}^{-1}$. HRMS (FD) $\mathrm{m} / z:[\mathrm{M}]^{+}$calcd for $\mathrm{C}_{21} \mathrm{H}_{29} \mathrm{O}_{3} \mathrm{P} 360.1854$; found: 360.1839 . 


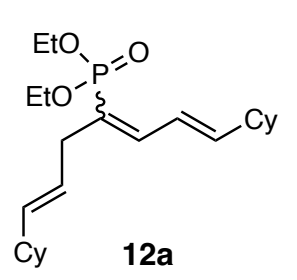

According to Procedure $\mathrm{B}$, an isomeric mixture of crude phosphonate 12a (3E/3Z $=1.4: 1.0)$ was obtained from $\mathbf{S} 7$ (176 $\mathrm{mg}, 0.645 \mathrm{mmol})$, diisopropylamine (191 $\mu \mathrm{L}, 1.35 \mathrm{mmol})$, a $2.67 \mathrm{M}$ solution of $n$-BuLi in $n$-hexane (506 $\mu \mathrm{L}, 1.35 \mathrm{mmol})$, diethyl chlorophosphate $(97.3 \mu \mathrm{L}, 0.677 \mathrm{mmol})$ and $(E)$-3-cyclohexylpropenal ${ }^{[5]}$ (93.6 mg, $0.677 \mathrm{mmol})$. Purification by flash column chromatography $\left(\mathrm{SiO}_{2}, n\right.$ hexane/EtOAc $=6: 1$ to 2:1) afforded (3E)-12a (104 mg, $0.264 \mathrm{mmol}, 41 \%)$ and (3Z)-12a (77.4 $\mathrm{mg}$, $0.196 \mathrm{mmol}, 30 \%)$.

(3E)-12a: pale yellow oil. ${ }^{1} \mathrm{H}$ NMR $\left(500 \mathrm{MHz}, \mathrm{CDCl}_{3}\right) \delta 6.98(\mathrm{dd}, J=22.4,10.9 \mathrm{~Hz}, 1 \mathrm{H}), 6.28$ (br dd, $J=14.9,10.9 \mathrm{~Hz}, 1 \mathrm{H}), 5.99$ (dd, $J=14.9,6.9 \mathrm{~Hz}, 1 \mathrm{H}), 5.42$ (dd, $J=15.5,6.9 \mathrm{~Hz}, 1 \mathrm{H}), 5.32$ (dt, $J=15.5,6.3 \mathrm{~Hz}, 1 \mathrm{H}), 4.10-3.94(\mathrm{~m}, 4 \mathrm{H}), 2.98$ (dd, $J=18.3,6.3 \mathrm{~Hz}, 2 \mathrm{H}), 2.13-2.03$ (m, 1H), 1.95$1.86(\mathrm{~m}, 1 \mathrm{H}), 1.77-1.63(\mathrm{~m}, 10 \mathrm{H}), 1.32-0.98(\mathrm{~m}, 16 \mathrm{H}) .{ }^{13} \mathrm{C} \mathrm{NMR}\left(126 \mathrm{MHz}, \mathrm{CDCl}_{3}\right) \quad \delta \quad 148.8$, $144.3\left(\mathrm{~d}, J_{\mathrm{C}-\mathrm{P}}=12.0 \mathrm{~Hz}\right), 137.7,124.3\left(\mathrm{~d}, J_{\mathrm{C}-\mathrm{P}}=180 \mathrm{~Hz}\right), 123.9\left(\mathrm{~d}, J_{\mathrm{C}-\mathrm{P}}=3.6 \mathrm{~Hz}\right), 122.5\left(\mathrm{~d}, J_{\mathrm{C}-\mathrm{P}}=\right.$ $22.8 \mathrm{~Hz}), 61.3\left(\mathrm{~d}, J_{\mathrm{C}-\mathrm{P}}=4.8 \mathrm{~Hz}, 2 \mathrm{C}\right), 41.1,40.6,32.9(2 \mathrm{C}), 32.3(2 \mathrm{C}), 30.4\left(\mathrm{~d}, J_{\mathrm{C}-\mathrm{P}}=9.6 \mathrm{~Hz}\right), 26.1$, 26.0, 25.9 (2C), $25.8(2 \mathrm{C}), 16.3\left(\mathrm{~d}, J_{\mathrm{C}-\mathrm{P}}=6.0 \mathrm{~Hz}, 2 \mathrm{C}\right) .{ }^{31} \mathrm{P} \mathrm{NMR}\left(202 \mathrm{MHz}, \mathrm{CDCl}_{3}\right) \delta 23.4$. IR (ATR) $v$ 2922, 1637, 1447, 1247, 1023, $963 \mathrm{~cm}^{-1}$. HRMS (FD) $\mathrm{m} / \mathrm{z}$ : $[\mathrm{M}]^{+}$calcd for $\mathrm{C}_{23} \mathrm{H}_{39} \mathrm{O}_{3} \mathrm{P} 394.2637$; found: 394.2620 .

(3Z)-12a: pale yellow oil. ${ }^{1} \mathrm{H} \mathrm{NMR}\left(500 \mathrm{MHz}, \mathrm{CDCl}_{3}\right) \delta 7.01(\mathrm{dd}, J=15.5,11.5 \mathrm{~Hz}, 1 \mathrm{H}), 6.58$ (dd, $J=48.7,11.5 \mathrm{~Hz}, 1 \mathrm{H}), 5.80(\mathrm{dd}, J=15.5,7.5 \mathrm{~Hz}, 1 \mathrm{H}), 5.44(\mathrm{dd}, J=15.5,6.9 \mathrm{~Hz}, 1 \mathrm{H}), 5.35(\mathrm{dt}, J=$ 15.5, $6.3 \mathrm{~Hz}, 1 \mathrm{H}), 4.12-3.97$ (m, 4H), 2.98 (dd, $J=12.1,6.3 \mathrm{~Hz}, 2 \mathrm{H}), 2.15-2.05(\mathrm{~m}, 1 \mathrm{H}), 1.99-1.90$ (m, 1H), 1.78-1.68 (m, 8H), 1.68-1.61 (m, 2H), 1.34-1.20 (m, 10H), $1.20-1.00(\mathrm{~m}, 6 \mathrm{H}) .{ }^{13} \mathrm{C} \mathrm{NMR}$ $\left(126 \mathrm{MHz}, \mathrm{CDCl}_{3}\right) \delta 147.5,145.5\left(\mathrm{~d}, J_{\mathrm{C}-\mathrm{P}}=10.8 \mathrm{~Hz}\right), 139.0,125.2\left(\mathrm{~d}, J_{\mathrm{C}-\mathrm{P}}=7.2 \mathrm{~Hz}\right), 125.0\left(\mathrm{~d}, J_{\mathrm{C}-\mathrm{P}}\right.$ $=173 \mathrm{~Hz}), 124.4\left(\mathrm{~d}, J_{\mathrm{C}-\mathrm{P}}=6.0 \mathrm{~Hz}\right), 61.2\left(\mathrm{~d}, J_{\mathrm{C}-\mathrm{P}}=4.8 \mathrm{~Hz}, 2 \mathrm{C}\right), 41.0,40.6,37.3\left(\mathrm{~d}, J_{\mathrm{C}-\mathrm{P}}=12.0 \mathrm{~Hz}\right)$, 33.0 (2C), 32.5 (2C), 26.1, 26.0 (overlapped, $2 \mathrm{C}+1 \mathrm{C}$ ), $25.8(2 \mathrm{C}), 16.3\left(\mathrm{~d}, J_{\mathrm{C}-\mathrm{P}}=7.2 \mathrm{~Hz}, 2 \mathrm{C}\right) .{ }^{31} \mathrm{P}$ NMR (202 MHz, $\mathrm{CDCl}_{3}$ ) $\delta$ 19.9. IR (ATR) v 2922, 2850, 1636, 1447, 1241, $963 \mathrm{~cm}^{-1}$. HRMS (FD) $m / z:[\mathrm{M}]^{+}$calcd for $\mathrm{C}_{23} \mathrm{H}_{39} \mathrm{O}_{3} \mathrm{P}$ 394.2637; found: 394.2646 .

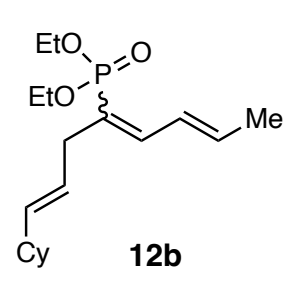

According to Procedure B, an isomeric mixture of crude phosphonate 12b $(3 E / 3 Z$ = 1.2:1.0) was obtained from $\mathbf{S} 7$ (134 $\mathrm{mg}, 0.488 \mathrm{mmol})$, diisopropylamine (145 $\mu \mathrm{L}, 1.03 \mathrm{mmol})$, a $2.69 \mathrm{M}$ solution of $n$-BuLi in $n$-hexane (381 $\mu \mathrm{L}, 1.03 \mathrm{mmol})$, diethyl chlorophosphate $(73.8 \mu \mathrm{L}, 0.513 \mathrm{mmol})$ and crotonaldehyde $(42.5 \mu \mathrm{L}$, 0.513 mmol). Purification by flash column chromatography $\left(\mathrm{SiO}_{2}, n\right.$ hexane/EtOAc $=2: 1$ to 1:1) afforded phosphonate (3E)-12b (72.0 mg, $0.221 \mathrm{mmol}, 45 \%)$ and (3Z)12b (62.5 mg, $0.191 \mathrm{mmol}, 39 \%)$.

(3E)-12b: colorless oil. ${ }^{1} \mathrm{H}$ NMR $\left(500 \mathrm{MHz}, \mathrm{CDCl}_{3}\right) \delta 6.99(\mathrm{dd}, J=22.9,11.5 \mathrm{~Hz}, 1 \mathrm{H}), 6.35$ (br dd, $J=14.5,11.5 \mathrm{~Hz}, 1 \mathrm{H}), 6.04(\mathrm{dq}, J=14.5,6.9 \mathrm{~Hz}, 1 \mathrm{H}), 5.43(\mathrm{dd}, J=15.5,6.9 \mathrm{~Hz}, 1 \mathrm{H}), 5.32(\mathrm{dt}, J=$ 15.5, 6.3 Hz, 1H), 4.13-3.94 (m, 4H), 2.98 (dd, $J=17.8,6.3 \mathrm{~Hz}, 2 \mathrm{H}), 1.96-1.87$ (m, 1H), 1.85 (d, $J$ 
$=6.9 \mathrm{~Hz}, 3 \mathrm{H}), 1.74-1.60(\mathrm{~m}, 5 \mathrm{H}), 1.36-1.19(\mathrm{~m}, 8 \mathrm{H}), 1.14(\mathrm{tt}, J=12.3,2.3 \mathrm{~Hz}, 1 \mathrm{H}), 1.08-0.98(\mathrm{~m}$, 2H). ${ }^{13} \mathrm{C}$ NMR $\left(126 \mathrm{MHz}, \mathrm{CDCl}_{3}\right) \quad \delta 143.8\left(\mathrm{~d}, J_{\mathrm{C}-\mathrm{P}}=10.8 \mathrm{~Hz}\right), 137.9,137.7,126.5\left(\mathrm{~d}, J_{\mathrm{C}-\mathrm{P}}=22.8\right.$ $\mathrm{Hz}), 124.1\left(\mathrm{~d}, J_{\mathrm{C}-\mathrm{P}}=180 \mathrm{~Hz}\right), 123.9\left(\mathrm{~d}, J_{\mathrm{C}-\mathrm{P}}=2.4 \mathrm{~Hz}\right), 61.4\left(\mathrm{~d}, J_{\mathrm{C}-\mathrm{P}}=4.8 \mathrm{~Hz}, 2 \mathrm{C}\right), 40.5,32.9(2 \mathrm{C})$, $30.4\left(\mathrm{~d}, J_{\mathrm{C}-\mathrm{P}}=9.6 \mathrm{~Hz}\right), 26.1,26.0(2 \mathrm{C}), 18.7,16.2\left(\mathrm{~d}, J_{\mathrm{C}-\mathrm{P}}=6.0 \mathrm{~Hz}, 2 \mathrm{C}\right) .{ }^{31} \mathrm{P} \mathrm{NMR}\left(202 \mathrm{MHz}, \mathrm{CDCl}_{3}\right)$ $\delta$ 23.4. IR (ATR) $\vee 2923,1644,1446,1246,1022,962 \mathrm{~cm}^{-1}$. HRMS (FD) $m / z:[\mathrm{M}]^{+}$calcd for $\mathrm{C}_{18} \mathrm{H}_{31} \mathrm{O}_{3} \mathrm{P} 326.2011$; found: 326.2003 .

(3Z)-12b: colorless oil. ${ }^{1} \mathrm{H}$ NMR (500 MHz, $\left.\mathrm{CDCl}_{3}\right) \delta 7.07$ (br dd, $\left.J=14.0,10.9 \mathrm{~Hz}, 1 \mathrm{H}\right), 6.59$ (dd, $J=48.7,10.9 \mathrm{~Hz}, 1 \mathrm{H}), 5.89(\mathrm{dq}, J=14.0,6.9 \mathrm{~Hz}, 1 \mathrm{H}), 5.44(\mathrm{dd}, J=15.5,6.3 \mathrm{~Hz}, 1 \mathrm{H}), 5.35(\mathrm{dt}, J$ $=15.5,6.9 \mathrm{~Hz}, 1 \mathrm{H}), 4.12-3.98(\mathrm{~m}, 4 \mathrm{H}), 2.90(\mathrm{dd}, J=12.0,6.3 \mathrm{~Hz}, 2 \mathrm{H}), 1.98-1.89(\mathrm{~m}, 1 \mathrm{H}), 1.83(\mathrm{~d}$, $J=6.9 \mathrm{~Hz}, 3 \mathrm{H}), 1.74-1.67(\mathrm{~m}, 4 \mathrm{H}), 1.67-1.60(\mathrm{~m}, 1 \mathrm{H}), 1.31(\mathrm{t}, J=6.9 \mathrm{~Hz}, 6 \mathrm{H}), 1.28-1.20(\mathrm{~m}, 2 \mathrm{H})$, $1.15(\mathrm{tt}, J=12.6,2.9 \mathrm{~Hz}, 1 \mathrm{H}), 1.11-1.01(\mathrm{~m}, 2 \mathrm{H}) .{ }^{13} \mathrm{C} \mathrm{NMR}\left(126 \mathrm{MHz}, \mathrm{CDCl}_{3}\right) \delta 145.2\left(\mathrm{~d}, J_{\mathrm{C}-\mathrm{P}}=\right.$ $12.0 \mathrm{~Hz}), 138.9,136.6,128.9\left(\mathrm{~d}, J_{\mathrm{C}-\mathrm{P}}=8.4 \mathrm{~Hz}\right), 124.6\left(\mathrm{~d}, J_{\mathrm{C}-\mathrm{P}}=173 \mathrm{~Hz}\right), 124.4\left(\mathrm{~d}, J_{\mathrm{C}-\mathrm{P}}=6.0 \mathrm{~Hz}\right)$, $61.2\left(\mathrm{~d}, J_{\mathrm{C}-\mathrm{P}}=4.8 \mathrm{~Hz}, 2 \mathrm{C}\right), 40.6,37.1\left(\mathrm{~d}, J_{\mathrm{C}-\mathrm{P}}=10.8 \mathrm{~Hz}\right), 33.0(2 \mathrm{C}), 26.1,26.0(2 \mathrm{C}), 18.4,16.3(\mathrm{~d}$, $\left.J_{\mathrm{C}-\mathrm{P}}=6.0 \mathrm{~Hz}, 2 \mathrm{C}\right) .{ }^{31} \mathrm{P} \mathrm{NMR}\left(202 \mathrm{MHz}, \mathrm{CDCl}_{3}\right) \delta 19.8$. IR (ATR) $v 2923,1642,1446,1239,1022$, $958 \mathrm{~cm}^{-1}$. HRMS (FD) $\mathrm{m} / z$ : [M] $]^{+}$calcd for $\mathrm{C}_{18} \mathrm{H}_{31} \mathrm{O}_{3} \mathrm{P}$ 326.2011; found: 326.2005 .

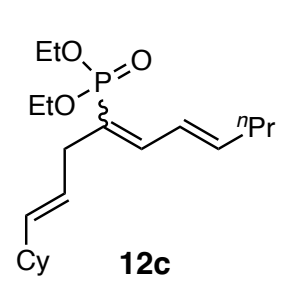

According to Procedure $\mathrm{B}$, an isomeric mixture of crude phosphonate $12 \mathrm{c}(3 E / 3 Z$ $=1.3: 1.0)$ was obtained from $\mathbf{S} 7(137 \mathrm{mg}, 0.500 \mathrm{mmol})$, diisopropylamine (148 $\mu \mathrm{L}, 1.05 \mathrm{mmol})$, a $2.69 \mathrm{M}$ solution of $n$-BuLi in $n$-hexane $(390 \mu \mathrm{L}, 1.05 \mathrm{mmol})$, diethyl chlorophosphate $(75.5 \mu \mathrm{L}, 0.525 \mathrm{mmol})$ and $(E)$-2-hexenal $(60.9 \mu \mathrm{L}$, $0.525 \mathrm{mmol})$. Purification by flash column chromatography $\left(\mathrm{SiO}_{2}, n\right.$ hexane/EtOAc $=4: 1$ to 2:1) afforded phosphonate ( $3 E)-12 c(74.5 \mathrm{mg}, 0.210 \mathrm{mmol}, 42 \%)$ and (3Z)12c (56.2 mg, $0.159 \mathrm{mmol}, 32 \%)$.

(3E)-12c: yellow oil. ${ }^{1} \mathrm{H}$ NMR $\left(500 \mathrm{MHz}, \mathrm{CDCl}_{3}\right) \delta 6.99(\mathrm{dd}, J=22.4,10.9 \mathrm{~Hz}, 1 \mathrm{H}), 6.33$ (br dd, $J$ $=14.3,10.9 \mathrm{~Hz}, 1 \mathrm{H}), 6.04(\mathrm{dt}, J=14.3,7.2 \mathrm{~Hz}, 1 \mathrm{H}), 5.42(\mathrm{dd}, J=15.5,6.9 \mathrm{~Hz}, 1 \mathrm{H}), 5.32(\mathrm{dt}, J=$ 15.5, 5.7 Hz, 1H), 4.10-3.94 (m, 4H), 2.98 (dd, $J=17.8,5.7 \mathrm{~Hz}, 2 \mathrm{H}), 2.15(\mathrm{td}, J=7.3,7.2 \mathrm{~Hz}, 2 \mathrm{H})$, 1.95-1.86 (m, 1H), 1.73-1.62 (m, 5H), $1.45(\mathrm{tq}, J=7.3,7.2 \mathrm{~Hz}, 2 \mathrm{H}), 1.30(\mathrm{t}, J=7.2 \mathrm{~Hz}, 6 \mathrm{H}), 1.26-$ $1.18(\mathrm{~m}, 2 \mathrm{H}), 1.14$ (br dd, $J=12.3,12.3 \mathrm{~Hz}, 1 \mathrm{H}), 1.07-0.98(\mathrm{~m}, 2 \mathrm{H}), 0.92(\mathrm{t}, J=7.2 \mathrm{~Hz}, 3 \mathrm{H}) .{ }^{13} \mathrm{C}$ $\operatorname{NMR}\left(126 \mathrm{MHz}, \mathrm{CDCl}_{3}\right) \delta 144.0\left(\mathrm{~d}, J_{\mathrm{C}-\mathrm{P}}=12.0 \mathrm{~Hz}\right), 143.1,137.7,125.2\left(\mathrm{~d}, J_{\mathrm{C}-\mathrm{P}}=22.8 \mathrm{~Hz}\right), 124.2$ $\left(\mathrm{d}, J_{\mathrm{C}-\mathrm{P}}=180 \mathrm{~Hz}\right), 123.9\left(\mathrm{~d}, J_{\mathrm{C}-\mathrm{P}}=2.4 \mathrm{~Hz}\right), 61.3\left(\mathrm{~d}, J_{\mathrm{C}-\mathrm{P}}=6.0 \mathrm{~Hz}, 2 \mathrm{C}\right), 40.5,35.0\left(\mathrm{~d}, J_{\mathrm{C}-\mathrm{P}}=2.4 \mathrm{~Hz}\right)$, $32.9(2 \mathrm{C}), 30.4\left(\mathrm{~d}, J_{\mathrm{C}-\mathrm{P}}=9.6 \mathrm{~Hz}\right), 26.1,25.9(2 \mathrm{C}), 21.9,16.3\left(\mathrm{~d}, J_{\mathrm{C}-\mathrm{P}}=6.0 \mathrm{~Hz}, 2 \mathrm{C}\right), 13.6 .{ }^{31} \mathrm{P} \mathrm{NMR}$ $\left(202 \mathrm{MHz}, \mathrm{CDCl}_{3}\right) \delta 23.4$. IR (ATR) $\vee 2924,1639,1447,1245,1023,963 \mathrm{~cm}^{-1}$. HRMS (FD) $\mathrm{m} / z$ : $[\mathrm{M}]^{+}$calcd for $\mathrm{C}_{20} \mathrm{H}_{35} \mathrm{O}_{3} \mathrm{P} 354.2324$; found: 354.2333 .

(3Z)-12c: pale yellow oil. ${ }^{1} \mathrm{H}$ NMR (500 MHz, $\left.\mathrm{CDCl}_{3}\right) \delta 7.03(\mathrm{dd}, J=14.6,11.5 \mathrm{~Hz}, 1 \mathrm{H}), 6.59$ (dd, $J=48.7,11.5 \mathrm{~Hz}, 1 \mathrm{H}), 5.87(\mathrm{dt}, J=14.6,7.3 \mathrm{~Hz}, 1 \mathrm{H}), 5.44(\mathrm{dd}, J=15.5,6.9 \mathrm{~Hz}, 1 \mathrm{H}), 5.35(\mathrm{dt}, J=$ 15.5, 6.3 Hz, 1H), 4.10-3.98 (m, 4H), 2.91 (dd, $J=12.1,6.3 \mathrm{~Hz}, 2 \mathrm{H}), 2.14(\mathrm{td}, J=7.4,7.3 \mathrm{~Hz}, 2 \mathrm{H})$, 
$1.99-1.90(\mathrm{~m}, 1 \mathrm{H}), 1.74-1.61(\mathrm{~m}, 5 \mathrm{H}), 1.44(\mathrm{tq}, J=7.4,7.4 \mathrm{~Hz}, 2 \mathrm{H}), 1.31$ (t, $J=7.2 \mathrm{~Hz}, 6 \mathrm{H}), 1.29-$ $1.20(\mathrm{~m}, 2 \mathrm{H}), 1.15$ (br dd, $J=12.3,12.3 \mathrm{~Hz}, 1 \mathrm{H}), 1.10-1.02(\mathrm{~m}, 2 \mathrm{H}), 0.91(\mathrm{t}, J=7.4 \mathrm{~Hz}, 3 \mathrm{H}) .{ }^{13} \mathrm{C}$ $\operatorname{NMR}\left(126 \mathrm{MHz}, \mathrm{CDCl}_{3}\right) \delta 145.2\left(\mathrm{~d}, J_{\mathrm{C}-\mathrm{P}}=12.0 \mathrm{~Hz}\right), 141.8,139.0,127.7\left(\mathrm{~d}, J_{\mathrm{C}-\mathrm{P}}=7.2 \mathrm{~Hz}\right), 124.9$ $\left(\mathrm{d}, J_{\mathrm{C}-\mathrm{P}}=173 \mathrm{~Hz}\right), 124.4\left(\mathrm{~d}, J_{\mathrm{C}-\mathrm{P}}=6.0 \mathrm{~Hz}\right), 61.2\left(\mathrm{~d}, J_{\mathrm{C}-\mathrm{P}}=6.0 \mathrm{~Hz}, 2 \mathrm{C}\right), 40.6,37.2\left(\mathrm{~d}, J_{\mathrm{C}-\mathrm{P}}=10.8\right.$ $\mathrm{Hz}$ ), 34.8, 33.0 (2C), 26.1, 26.0 (2C), 22.1, 16.3 (d, $\left.J_{\mathrm{C}-\mathrm{P}}=7.2 \mathrm{~Hz}, 2 \mathrm{C}\right), 13.7 .{ }^{31} \mathrm{P}$ NMR (202 MHz, $\left.\mathrm{CDCl}_{3}\right) \delta$ 19.9. IR (ATR) $\vee 2924,1638,1447,1241,1022,962 \mathrm{~cm}^{-1}$. HRMS (FD) $\mathrm{m} / z:[\mathrm{M}]^{+}$calcd for $\mathrm{C}_{20} \mathrm{H}_{35} \mathrm{O}_{3} \mathrm{P}$ 354.2324; found: 354.2313 .

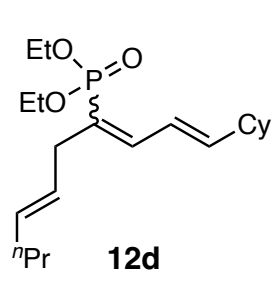

According to Procedure B, an isomeric mixture of crude phosphonate 12d $(3 E / 3 Z$ = 1.5:1.0) was obtained from $\mathbf{S 8}(117 \mathrm{mg}, 0.500 \mathrm{mmol})$, diisopropylamine (148 $\mu \mathrm{L}, 1.05 \mathrm{mmol})$, a $2.69 \mathrm{M}$ solution of $n$-BuLi in $n$-hexane (390 $\mu \mathrm{L}, 1.05 \mathrm{mmol})$, diethyl chlorophosphate $(75.5 \mu \mathrm{L}, 0.525 \mathrm{mmol})$ and $(E)$-3-cyclohexylpropenal ${ }^{[5]}$ (72.6 mg, $0.525 \mathrm{mmol}$ ). Purification by flash column chromatography $\left(\mathrm{SiO}_{2}, n\right.$ hexane/EtOAc $=4: 1$ to $1: 1)$ afforded phosphonate (3E)-12d $(89.8 \mathrm{mg}, 0.253 \mathrm{mmol}, 51 \%)$ and (3Z)12d (62.3 mg, $0.176 \mathrm{mmol}, 35 \%)$.

(3E)-12d: pale yellow oil. ${ }^{1} \mathrm{H}$ NMR $\left(500 \mathrm{MHz}, \mathrm{CDCl}_{3}\right) \delta 6.99(\mathrm{dd}, J=22.4,10.9 \mathrm{~Hz}, 1 \mathrm{H}), 6.29$ (br dd, $J=14.9,10.9 \mathrm{~Hz}, 1 \mathrm{H}), 5.99$ (dd, $J=14.9,6.9 \mathrm{~Hz}, 1 \mathrm{H}), 5.47$ (dt, $J=15.5,6.9 \mathrm{~Hz}, 1 \mathrm{H}), 5.38$ (dt, $J=15.5,6.3 \mathrm{~Hz}, 1 \mathrm{H}), 4.10-3.94(\mathrm{~m}, 4 \mathrm{H}), 2.99$ (dd, $J=17.8,6.3 \mathrm{~Hz}, 2 \mathrm{H}), 2.14-2.03(\mathrm{~m}, 1 \mathrm{H}), 1.96$ $(\mathrm{td}, J=7.3,6.9 \mathrm{~Hz}, 2 \mathrm{H}), 1.78-1.70(\mathrm{~m}, 4 \mathrm{H}), 1.67-1.55(\mathrm{~m}, 2 \mathrm{H}), 1.40-1.23(\mathrm{~m}, 10 \mathrm{H}), 1.22-1.08(\mathrm{~m}$, $2 \mathrm{H}), 0.87(\mathrm{t}, J=7.3 \mathrm{~Hz}, 3 \mathrm{H}) .{ }^{13} \mathrm{C} \mathrm{NMR}\left(126 \mathrm{MHz}, \mathrm{CDCl}_{3}\right) \delta 148.9,144.3\left(\mathrm{~d}, J_{\mathrm{C}-\mathrm{P}}=12.0 \mathrm{~Hz}\right), 131.6$, $126.6\left(\mathrm{~d}, J_{\mathrm{C}-\mathrm{P}}=2.4 \mathrm{~Hz}\right), 124.3\left(\mathrm{~d}, J_{\mathrm{C}-\mathrm{P}}=181 \mathrm{~Hz}\right), 122.5\left(\mathrm{~d}, J_{\mathrm{C}-\mathrm{P}}=22.8 \mathrm{~Hz}\right), 61.4\left(\mathrm{~d}, J_{\mathrm{C}-\mathrm{P}}=6.0 \mathrm{~Hz}\right.$, $2 \mathrm{C}), 41.1,34.5,32.3(2 \mathrm{C}), 30.4\left(\mathrm{~d}, J_{\mathrm{C}-\mathrm{P}}=10.8 \mathrm{~Hz}\right), 26.0,25.8,22.5(2 \mathrm{C}), 16.3\left(\mathrm{~d}, J_{\mathrm{C}-\mathrm{P}}=7.2 \mathrm{~Hz}, 2 \mathrm{C}\right)$, 13.6. ${ }^{31} \mathrm{P}$ NMR $\left(202 \mathrm{MHz} \mathrm{CDCl}_{3}\right) \delta$ 23.1. IR (ATR) $\vee 2925,1637,1248,1024,960 \mathrm{~cm}^{-1}$. HRMS (FD) $m / z:[\mathrm{M}]^{+}$calcd for $\mathrm{C}_{20} \mathrm{H}_{35} \mathrm{O}_{3} \mathrm{P}$ 354.2324; found: 354.2311 .

(3Z)-12d: pale yellow oil. ${ }^{1} \mathrm{H}$ NMR $\left(500 \mathrm{MHz}, \mathrm{CDCl}_{3}\right) \delta 7.01(\mathrm{dd}, J=14.9,10.9 \mathrm{~Hz}, 1 \mathrm{H}), 6.59$ (dd, $J=48.1,10.9 \mathrm{~Hz}, 1 \mathrm{H}), 5.80(\mathrm{dt}, J=14.9,7.5 \mathrm{~Hz}, 1 \mathrm{H}), 5.48(\mathrm{dt}, J=15.5,6.3 \mathrm{~Hz}, 1 \mathrm{H}), 5.40(\mathrm{dt}, J=$ $14.9,6.9 \mathrm{~Hz}, 1 \mathrm{H}), 4.12-3.98(\mathrm{~m}, 4 \mathrm{H}), 2.92(\mathrm{dd}, J=11.5,6.3 \mathrm{~Hz}, 2 \mathrm{H}), 2.14-2.05(\mathrm{~m}, 1 \mathrm{H}), 1.99$ (td, $J=7.3,6.9 \mathrm{~Hz}, 2 \mathrm{H}), 1.77-1.68(\mathrm{~m}, 4 \mathrm{H}), 1.67-1.61(\mathrm{~m}, 1 \mathrm{H}), 1.39$ (qt, $J=7.5,7.3 \mathrm{~Hz}, 2 \mathrm{H}), 1.32-$ $1.20(\mathrm{~m}, 8 \mathrm{H}), 1.20-1.06(\mathrm{~m}, 3 \mathrm{H}), 0.90(\mathrm{t}, J=7.5 \mathrm{~Hz}, 3 \mathrm{H}) .{ }^{13} \mathrm{C} \mathrm{NMR}\left(126 \mathrm{MHz}, \mathrm{CDCl}_{3}\right) \delta 147.5$, $145.5\left(\mathrm{~d}, J_{\mathrm{C}-\mathrm{P}}=12.0 \mathrm{~Hz}\right), 132.9,127.1\left(\mathrm{~d}, J_{\mathrm{C}-\mathrm{P}}=6.0 \mathrm{~Hz}\right), 125.2\left(\mathrm{~d}, J_{\mathrm{C}-\mathrm{P}}=7.2 \mathrm{~Hz}\right), 124.9\left(\mathrm{~d}, J_{\mathrm{C}-\mathrm{P}}=\right.$ $173 \mathrm{~Hz}), 61.2\left(\mathrm{~d}, J_{\mathrm{C}-\mathrm{P}}=4.8 \mathrm{~Hz}, 2 \mathrm{C}\right), 41.0,37.3\left(\mathrm{~d}, J_{\mathrm{C}-\mathrm{P}}=10.8 \mathrm{~Hz}\right), 34.6,32.5(2 \mathrm{C}), 26.0,25.8,22.5$ $(2 \mathrm{C}), 16.3\left(\mathrm{~d}, J_{\mathrm{C}-\mathrm{P}}=6.0 \mathrm{~Hz}, 2 \mathrm{C}\right), 13.7 .{ }^{31} \mathrm{P} \mathrm{NMR}\left(202 \mathrm{MHz}, \mathrm{CDCl}_{3}\right) \delta$ 19.9. IR (ATR) v 2925, 1637, 1242, 1023, $962 \mathrm{~cm}^{-1}$. HRMS (FD) $m / z$ : [M] calcd for $\mathrm{C}_{20} \mathrm{H}_{35} \mathrm{O}_{3} \mathrm{P}$ 354.2324; found: 354.2312 . 


\section{Anionic 8 $\pi$ Electrocyclic and Subsequent HWE Reactions of Stereoisomers of Triene 5}

(Scheme 3)<smiles>CCOP(=O)(/C=C/C=C/c1ccccc1)OCC</smiles>

$(1 E, 3 E)-5$
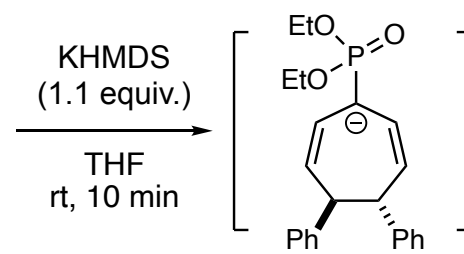

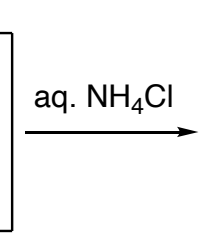

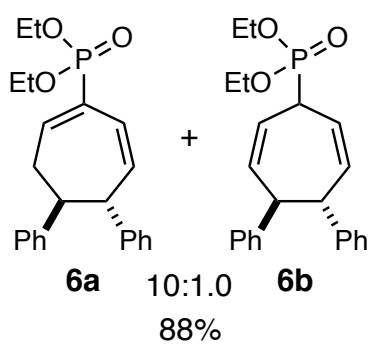

To a solution of $(1 E, 3 E)-5(38.2 \mathrm{mg}, 0.100 \mathrm{mmol})$ in THF $(1.0 \mathrm{~mL})$ at room temperature was added a $1.0 \mathrm{M}$ solution of KHMDS in THF $(110 \mu \mathrm{L}, 0.110 \mathrm{mmol})$. After stirring for $10 \mathrm{~min}$ at room temperature, the reaction was quenched with saturated aqueous $\mathrm{NH}_{4} \mathrm{Cl}(1 \mathrm{~mL})$. After EtOAc $(1 \mathrm{~mL})$ was added, the two layers were separated, and the aqueous layer was extracted with EtOAc $(2 \times 1$ $\mathrm{mL}$ ). The combined organic layers were dried over $\mathrm{MgSO}_{4}$, filtered, and concentrated under reduced pressure. Purification by flash column chromatography $\left(\mathrm{SiO}_{2}, n\right.$-hexane/EtOAc $=2: 1$ to $\left.1: 1\right)$ afforded trans-cyclic phosphonate $(33.6 \mathrm{mg}, 87.9 \mu \mathrm{mol}, 88 \%)$ as a mixture of regioisomers $(\mathbf{6 a}: 6 \mathbf{b}$ $=10: 1.0)$ regarding double bonds.

6a (major isomer): pale yellow oil. ${ }^{1} \mathrm{H} \mathrm{NMR}\left(500 \mathrm{MHz}, \mathrm{CDCl}_{3}\right) \delta 7.16-7.05(\mathrm{~m}, 7 \mathrm{H}), 6.94-6.89(\mathrm{~m}$, 4H), 6.20-6.09 (m, 2H), 4.15-3.94 (m, 4H), 3.87 (dd, $J=8.6,4.0 \mathrm{~Hz}, 1 \mathrm{H}), 3.31-3.24(\mathrm{~m}, 1 \mathrm{H}), 2.84$ (ddd, $J=15.5,8.0,8.0 \mathrm{~Hz}, 1 \mathrm{H}), 2.70$ (ddd, $J=15.5,6.9,2.9 \mathrm{~Hz}, 1 \mathrm{H}), 1.38$ (t, $J=7.2 \mathrm{~Hz}, 3 \mathrm{H}$ ), 1.33 $(\mathrm{t}, J=7.2 \mathrm{~Hz}, 3 \mathrm{H}) .{ }^{13} \mathrm{C} \mathrm{NMR}\left(126 \mathrm{MHz}, \mathrm{CDCl}_{3}\right) \delta 147.8\left(\mathrm{~d}, J_{\mathrm{C}-\mathrm{P}}=8.4 \mathrm{~Hz}\right), 145.1,143.4,138.4(\mathrm{~d}$, $\left.J_{\mathrm{C}-\mathrm{P}}=16.8 \mathrm{~Hz}\right), 128.42(2 \mathrm{C}), 128.36\left(\mathrm{~d}, J_{\mathrm{C}-\mathrm{P}}=180 \mathrm{~Hz}\right), 128.2(2 \mathrm{C}), 128.1(2 \mathrm{C}), 127.2(2 \mathrm{C}), 126.4$, $126.2,123.0\left(\mathrm{~d}, J_{\mathrm{C}-\mathrm{P}}=10.8 \mathrm{~Hz}\right), 61.9-61.7(\mathrm{~m}, 2 \mathrm{C}), 55.5,53.3\left(\mathrm{~d}, J_{\mathrm{C}-\mathrm{P}}=9.6 \mathrm{~Hz}\right), 35.3\left(\mathrm{~d}, J_{\mathrm{C}-\mathrm{P}}=20.4\right.$ $\mathrm{Hz}$ ), 16.5-16.3 (m, 2C). ${ }^{31} \mathrm{P}$ NMR (202 $\left.\mathrm{MHz}, \mathrm{CDCl}_{3}\right) \delta 20.1$. IR (ATR) v 2980, 1248, 1023, 961, $700 \mathrm{~cm}^{-1}$. HRMS (FD) $\mathrm{m} / z$ : [M] $]^{+}$calcd for $\mathrm{C}_{23} \mathrm{H}_{27} \mathrm{O}_{3} \mathrm{P} 382.1698$; found: 382.1682 .

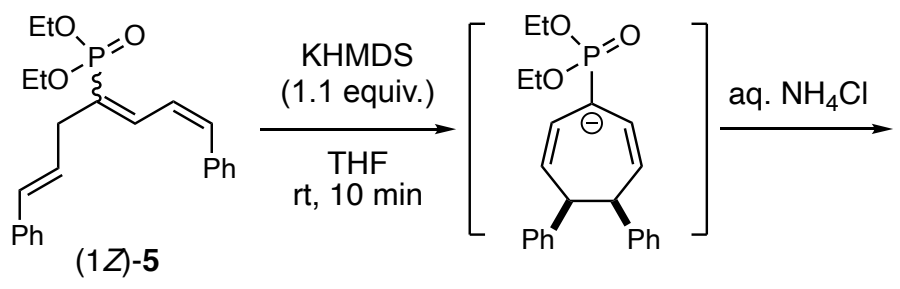

$3 E / 3 Z=1.0: 1.4$

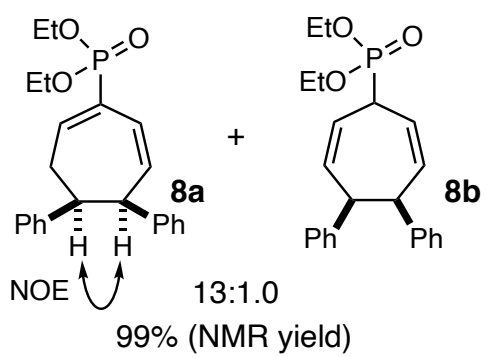

$99 \%$ (NMR yield)

To a solution of isomeric mixture of $(1 Z)-5(3 E / 3 Z=1.0: 1.4,38.2 \mathrm{mg}, 0.100 \mathrm{mmol})$ in THF $(1.0$ $\mathrm{mL})$ at room temperature was added a 1.0 M solution of KHMDS in THF (110 $\mu \mathrm{L}, 0.110 \mathrm{mmol})$. After stirring for $10 \mathrm{~min}$ at room temperature, the mixture was cooled to $0{ }^{\circ} \mathrm{C}$ and saturated aqueous $\mathrm{NH}_{4} \mathrm{Cl}(1 \mathrm{~mL})$ was added immediately. After EtOAc $(1 \mathrm{~mL})$ was added, the two layers were separated, and the aqueous layer was extracted with EtOAc $(2 \times 1 \mathrm{~mL})$. The combined organic layers were dried over $\mathrm{MgSO}_{4}$, filtered, and concentrated under reduced pressure. NMR measurement with 
internal pyrazine $(10.0 \mathrm{mg})$ elucidated an obtained crude product to contain cis-cyclic phosphonate $(\mathbf{8 a}+\mathbf{8 b})(99 \%$ NMR yield $)$ as a mixture of regioisomers $(\mathbf{8 a}: \mathbf{8 b}=13: 1.0)$ regarding double bonds.

8a: pale yellow oil. ${ }^{1} \mathrm{H}$ NMR $\left(500 \mathrm{MHz}, \mathrm{CDCl}_{3}\right) \delta$ 7.20-7.05 (m, 7H), 6.79-6.76 (m, 2H), $6.62(\mathrm{~d}$, $J=6.9 \mathrm{~Hz}, 2 \mathrm{H}), 6.28(\mathrm{dd}, J=11.5,11.2 \mathrm{~Hz}, 1 \mathrm{H}), 6.18(\mathrm{ddd}, J=11.5,6.9,2.9 \mathrm{~Hz}, 1 \mathrm{H}), 4.24-4.09$ (m, 4H), 3.87-3.82 (m, 1H), $3.48(\mathrm{ddd}, J=9.2,2.6,2.3 \mathrm{~Hz}, 1 \mathrm{H}), 2.83-2.72(\mathrm{~m}, 1 \mathrm{H}), 2.62(\mathrm{ddd}, J=$ 18.4, 8.1, $2.3 \mathrm{~Hz}, 1 \mathrm{H}), 1.42-1.35(\mathrm{~m}, 6 \mathrm{H}) .{ }^{13} \mathrm{C} \mathrm{NMR}\left(126 \mathrm{MHz}, \mathrm{CDCl}_{3}\right) \delta 146.8\left(\mathrm{~d}, J_{\mathrm{C}-\mathrm{P}}=8.4 \mathrm{~Hz}\right)$, $143.2,139.5,136.8\left(\mathrm{~d}, J_{\mathrm{C}-\mathrm{P}}=18.0 \mathrm{~Hz}\right), 130.4(2 \mathrm{C}), 128.1(2 \mathrm{C}), 127.8(2 \mathrm{C}), 127.7,127.5(2 \mathrm{C}), 126.8$, $126.3,122.4\left(\mathrm{~d}, J_{\mathrm{C}-\mathrm{P}}=10.8 \mathrm{~Hz}\right), 61.9-61.8(\mathrm{~m}, 2 \mathrm{C}), 54.4,46.0,33.2\left(\mathrm{~d}, J_{\mathrm{C}-\mathrm{P}}=21.6 \mathrm{~Hz}\right), 16.4\left(\mathrm{~d}, J_{\mathrm{C}-}\right.$ $\mathrm{P}=6.0 \mathrm{~Hz}, 2 \mathrm{C}) .{ }^{31} \mathrm{P} \mathrm{NMR}\left(202 \mathrm{MHz}, \mathrm{CDCl}_{3}\right) \delta 21.7$. IR (ATR) $\vee 2980,1247,1024,961,701 \mathrm{~cm}^{-1}$. HRMS (FD) $m / z$ : [M] $]^{+}$calcd for $\mathrm{C}_{23} \mathrm{H}_{27} \mathrm{O}_{3} \mathrm{P} 382.1698$; found: 382.1694 .

\section{General Procedure for $8 \pi$ Electrocyclization/HWE Reaction Sequence}

\section{Procedure C}

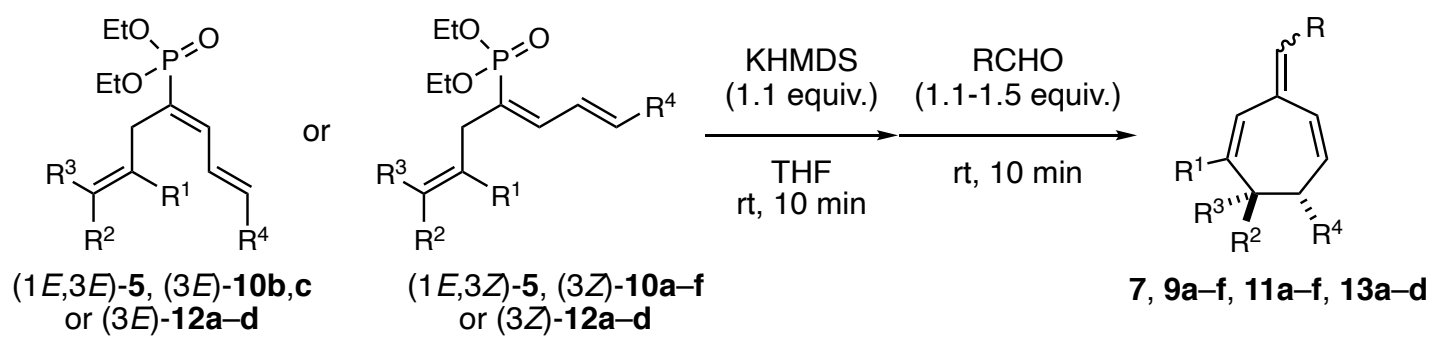

To a $0.1 \mathrm{M}$ solution of cyclization precursor in THF at room temperature was added a $1.0 \mathrm{M}$ solution of KHMDS in THF (1.1 equiv.). After stirring for $10 \mathrm{~min}$ at room temperature, aldehyde (1.5 equiv.) was added, and the resulting mixture was stirred for another $10 \mathrm{~min}$ at room temperature. Then, the reaction was quenched with saturated aqueous $\mathrm{NH}_{4} \mathrm{Cl}$. After EtOAc was added, the two layers were separated, and the aqueous layer was extracted with EtOAc (2 times). The combined organic layers were dried over $\mathrm{MgSO}_{4}$, filtered, and concentrated under reduced pressure. Purification by flash column chromatography $\left(\mathrm{SiO}_{2}, n\right.$-hexane/EtOAc $=1: 0$ to $\left.30: 1\right)$ afforded the corresponding cycloheptatriene. When cycloheptatriene was obtained as a mixture with benzaldehyde, benzaldehyde was azeotropically removed together with toluene (3 times). 


\section{Scope of Aldehydes in One-pot HWE Reactions (Figure 1)}

\section{3-Benzylidene-6,7-anti-diphenyl-1,4-cycloheptadiene (7)}

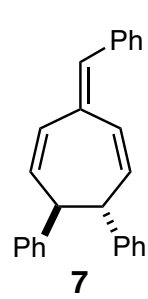

According to Procedure C, phosphonate (1E,3E)-5 (76.5 mg, $0.200 \mathrm{mmol})$ was converted to cycloheptatriene $7(58.8 \mathrm{mg}, 0.176 \mathrm{mmol}, 88 \%)$ by using a $1.0 \mathrm{M}$ solution of KHMDS in THF $(220 \mu \mathrm{L}, 0.220 \mathrm{mmol})$ and PhCHO (30.6 $\mu \mathrm{L}, 0.300 \mathrm{mmol})$. Also, (1E,3Z)-5 (38.2 $\mathrm{mg}, 0.100 \mathrm{mmol})$ was converted to $7(54.7 \mathrm{mg}, 0.164 \mathrm{mmol}, 82 \%)$ by using a $1.0 \mathrm{M}$ solution of KHMDS in THF $(110 \mu \mathrm{L}, 0.110 \mathrm{mmol})$ and PhCHO $(15.3 \mu \mathrm{L}, 0.150 \mathrm{mmol})$.

The reaction was also conducted at $1 \mathrm{mmol}$ scale. (1E,3Z)-5 (382 $\mathrm{mg}, 1.00 \mathrm{mmol})$ was converted to 7 (269 mg, $0.804 \mathrm{mmol}, 80 \%$ ) by using a 1.0 M solution of KHMDS in THF (1.10 mL, $1.10 \mathrm{mmol})$ and $\mathrm{PhCHO}(153 \mu \mathrm{L}, 1.50 \mathrm{mmol})$.

7: yellow oil. ${ }^{1} \mathrm{H}$ NMR $\left(500 \mathrm{MHz}, \mathrm{CDCl}_{3}\right) \delta 7.41(\mathrm{~d}, J=7.5 \mathrm{~Hz}, 2 \mathrm{H}), 7.37(\mathrm{dd}, J=7.5,7.5 \mathrm{~Hz}, 2 \mathrm{H})$, $7.27(\mathrm{t}, J=7.5 \mathrm{~Hz}, 1 \mathrm{H}), 7.22-7.08(\mathrm{~m}, 10 \mathrm{H}), 6.70(\mathrm{~s}, 1 \mathrm{H}), 6.68(\mathrm{~d}, J=12.1 \mathrm{~Hz}, 1 \mathrm{H}), 6.35(\mathrm{~d}, J=$ $12.1 \mathrm{~Hz}, 1 \mathrm{H}), 5.94$ (dd, $J=12.1,5.7 \mathrm{~Hz}, 1 \mathrm{H}), 5.78$ (dd, $J=12.1,5.2 \mathrm{~Hz}, 1 \mathrm{H}), 4.02$ (dd, $J=6.6,5.7$ $\mathrm{Hz}, 1 \mathrm{H}), 3.96(\mathrm{dd}, J=6.6,5.2 \mathrm{~Hz}, 1 \mathrm{H}) .{ }^{13} \mathrm{C} \mathrm{NMR}\left(126 \mathrm{MHz}, \mathrm{CDCl}_{3}\right) \delta 144.12,144.05,137.1,135.9$, 135.4 134.6, 132.6, 132.5, 129.9 (2C), 128.5 (2C), 128.3 (2C), 128.14 (overlapped, 2C + 2C), 128.09 (2C), 128.0, 127.6, 127.3, 126.1, 52.9, 52.6. IR (ATR) v 3019, 1597, 1489, $1449 \mathrm{~cm}^{-1}$. HRMS (FD) $\mathrm{m} / z$ : $[\mathrm{M}]^{+}$calcd for $\mathrm{C}_{26} \mathrm{H}_{22}$ 334.1722; found: 334.1720 .

\section{3-(4-Methoxybenzylidene)-6,7-anti-diphenyl-1,4-cycloheptadiene (9a)}

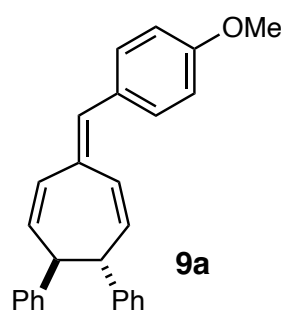

According to Procedure C, phosphonate (1E,3E)-5 (38.2 mg, $0.100 \mathrm{mmol})$ was converted to cycloheptatriene 9a (30.6 mg, $84.0 \mu \mathrm{mol}, 84 \%)$. Also, (1E,3Z)-5 $(38.2 \mathrm{mg}, 0.100 \mathrm{mmol})$ was converted to $9 \mathrm{a}(29.6 \mathrm{mg}, 81.2 \mu \mathrm{mol}, 81 \%)$ by using a $1.0 \mathrm{M}$ solution of KHMDS in THF $(110 \mu \mathrm{L}, 0.110 \mathrm{mmol}$, and $p$-anisaldehyde (18.2 $\mu \mathrm{L}, 0.150 \mathrm{mmol})$.

9a: pale yellow solid. Mp 87-91 ${ }^{\circ} \mathrm{C}$ ( $n$-hexane/EtOAc). ${ }^{1} \mathrm{H}$ NMR $\left(500 \mathrm{MHz}, \mathrm{CDCl}_{3}\right) \delta 7.36(\mathrm{~d}, J=$ $9.2 \mathrm{~Hz}, 2 \mathrm{H}), 7.20-7.06(\mathrm{~m}, 10 \mathrm{H}), 6.91(\mathrm{~d}, J=8.6 \mathrm{~Hz}, 2 \mathrm{H}), 6.69-6.63(\mathrm{~m}, 2 \mathrm{H}), 6.35(\mathrm{~d}, J=12.0 \mathrm{~Hz}$, $1 \mathrm{H}), 5.93(\mathrm{dd}, J=11.5,5.7 \mathrm{~Hz}, 1 \mathrm{H}), 5.73(\mathrm{dd}, J=12.0,5.2 \mathrm{~Hz}, 1 \mathrm{H}), 4.00(\mathrm{dd}, J=6.6,5.7 \mathrm{~Hz}, 1 \mathrm{H})$, $3.95(\mathrm{dd}, J=6.6,5.2 \mathrm{~Hz}, 1 \mathrm{H}), 3.83(\mathrm{~s}, 3 \mathrm{H}) .{ }^{13} \mathrm{C} \mathrm{NMR}\left(126 \mathrm{MHz}, \mathrm{CDCl}_{3}\right) \delta 159.0,144.3,144.2$, $135.1,134.5,134.2,132.7,131.8,131.3$ (2C), 129.8, 128.5 (2C), 128.3 (2C), 128.1 (2C), 128.0 (2C), $127.8,126.0,113.6(2 \mathrm{C}), 55.3,52.9,52.6$ (One signal could not be judged due to overlap to the signals among 126.0-135.1 ppm.). IR (ATR) v 3025, 1601, 1505, 1302, 1249, 1176, $1028 \mathrm{~cm}^{-1}$. HRMS (FD) $m / z$ : [M] $]^{+}$calcd for $\mathrm{C}_{27} \mathrm{H}_{24} \mathrm{O} 364.1827$; found: 364.1845 . 


\section{3-(4-Methoxycarbonylbenzylidene)-6,7-anti-diphenyl-1,4-cycloheptadiene (9b)}

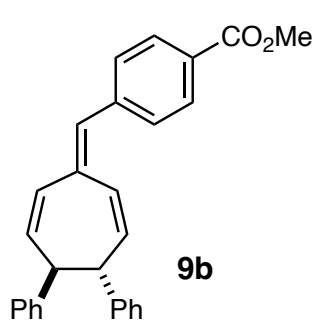

$0.150 \mathrm{mmol})$.

According to Procedure C, phosphonate (1E,3E)-5 (42.3mg, $0.111 \mathrm{mmol})$ was converted to cycloheptatriene $9 \mathbf{b}(30.2 \mathrm{mg}, 76.9 \mu \mathrm{mol}, 69 \%)$ by using a $1.0 \mathrm{M}$ solution of KHMDS in THF (122 $\mu \mathrm{L}, 0.122 \mathrm{mmol})$ and methyl-4formylbenzate $(20.0 \mathrm{mg}, 0.166 \mathrm{mmol})$. Also, (1E,3Z)-5 (38.2 mg, $0.100 \mathrm{mmol})$ was converted to $\mathbf{9 b}(31.3 \mathrm{mg}, 79.7 \mu \mathrm{mol}, 80 \%)$ by using a $1.0 \mathrm{M}$ solution of KHMDS in THF $(110 \mu \mathrm{L}, 0.110 \mathrm{mmol})$ and methyl 4-formylbenzate $(24.6 \mathrm{mg}$,

9b: pale yellow crystal. Mp $134-136{ }^{\circ} \mathrm{C}$ ( $n$-hexane/EtOAc). ${ }^{1} \mathrm{H}$ NMR $\left(500 \mathrm{MHz}, \mathrm{CDCl}_{3}\right) \delta 8.02(\mathrm{~d}$, $J=8.6 \mathrm{~Hz}, 2 \mathrm{H}), 7.46$ (d, $J=8.0 \mathrm{~Hz}, 2 \mathrm{H}), 7.21-7.07(\mathrm{~m}, 10 \mathrm{H}), 6.69(\mathrm{~s}, 1 \mathrm{H}), 6.63$ (d, $J=12.0 \mathrm{~Hz}$, $1 \mathrm{H}), 6.35(\mathrm{~d}, J=12.6 \mathrm{~Hz}, 1 \mathrm{H}), 6.00(\mathrm{dd}, J=12.0,6.3 \mathrm{~Hz}, 1 \mathrm{H}), 5.84(\mathrm{dd}, J=12.6,5.2 \mathrm{~Hz}, 1 \mathrm{H}), 4.03$ $(\mathrm{dd}, J=6.9,6.3 \mathrm{~Hz}, 1 \mathrm{H}), 3.96(\mathrm{dd}, J=6.9,5.2 \mathrm{~Hz}, 1 \mathrm{H}), 3.92(\mathrm{~s}, 3 \mathrm{H}) .{ }^{13} \mathrm{C} \mathrm{NMR}\left(126 \mathrm{MHz}, \mathrm{CDCl}_{3}\right)$ $\delta 166.8,143.81,143.76,141.7,137.6,135.9,134.0,133.7,132.3,129.8$ (2C), 129.4 (overlapped, 2C), 128.6, 128.4 (2C), 128.3 (2C), 128.13 (2C), 128.06 (2C), 127.1 (2C), 126.1, 52.8, 52.4, 52.1. IR (ATR) v 3025, 1709, 1599, 1276, 1182, $1104 \mathrm{~cm}^{-1}$. HRMS (FD) $m / z$ : [M] calcd for $\mathrm{C}_{28} \mathrm{H}_{24} \mathrm{O}_{2}$ 392.1777; found: 392.1772 .

\section{3-(4-Cyanobenzylidene)-6,7-anti-diphenyl-1,4-cycloheptadiene (9c)}

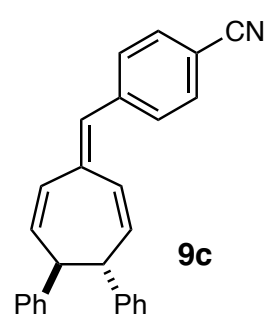

According to Procedure C, phosphonate $(1 E, 3 E)-5(38.2 \mathrm{mg}, 0.100 \mathrm{mmol})$ was converted to cycloheptatriene $9 \mathrm{c}(26.8 \mathrm{mg}, 74.6 \mu \mathrm{mol}, 75 \%)$, and (1E,3Z)-5 (38.2 $\mathrm{mg}, 0.100 \mathrm{mmol})$ was converted to $9 \mathrm{c}(27.7 \mathrm{mg}, 77.1 \mu \mathrm{mol}, 77 \%)$ by using a 1.0 M solution of KHMDS in THF $(110 \mu \mathrm{L}, 0.110 \mathrm{mmol})$ and 4-formylbenzonitrile (19.7 mg, $0.150 \mathrm{mmol})$.

9c: colorless crystal. Mp $145-147{ }^{\circ} \mathrm{C}\left(n\right.$-hexane/EtOAc). ${ }^{1} \mathrm{H}$ NMR $\left(500 \mathrm{MHz}, \mathrm{CDCl}_{3}\right) \delta 7.64(\mathrm{~d}, J$ $=8.0 \mathrm{~Hz}, 2 \mathrm{H}), 7.48(\mathrm{~d}, J=8.1 \mathrm{~Hz}, 2 \mathrm{H}), 7.21-7.15(\mathrm{~m}, 4 \mathrm{H}), 7.14-7.07(\mathrm{~m}, 6 \mathrm{H}), 6.64(\mathrm{~s}, 1 \mathrm{H}), 6.56$ $(\mathrm{d}, J=11.5 \mathrm{~Hz}, 1 \mathrm{H}), 6.34(\mathrm{~d}, J=12.6 \mathrm{~Hz}, 1 \mathrm{H}), 6.04(\mathrm{dd}, J=12.0,6.3 \mathrm{~Hz}, 1 \mathrm{H}), 5.87(\mathrm{dd}, J=12.0$, $4.6 \mathrm{~Hz}, 1 \mathrm{H}), 4.03(\mathrm{dd}, J=6.6,6.3 \mathrm{~Hz}, 1 \mathrm{H}), 3.97(\mathrm{dd}, J=6.6,4.6 \mathrm{~Hz}, 1 \mathrm{H}) .{ }^{13} \mathrm{C} \mathrm{NMR}(126 \mathrm{MHz}$, $\left.\mathrm{CDCl}_{3}\right) \delta 143.6,143.5,141.8,138.5,136.8$ (overlapped, 2C), 134.6, 132.8, 131.93, 131.88 (2C), 130.3, 128.4 (2C), 128.23 (2C), 128.17 (2C), 128.1 (2C), 126.7, $126.2(2 \mathrm{C}), 118.9,110.4,52.7,52.3$. IR $\left(\text { ATR) } v 2888,2225,1725,1598,1489,1448,1180,1072 \mathrm{~cm}^{-1} \text {. HRMS (FD) } \mathrm{m} / z \text { : [M] }\right]^{+}$calcd for $\mathrm{C}_{27} \mathrm{H}_{21} \mathrm{~N} 359.1674$; found: 359.1658 . 


\section{3-(2-Bromobenzylidene)-6,7-anti-diphenyl-1,4-cycloheptadiene (9d)}

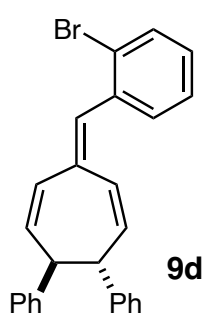

For the synthesis of cycloheptatriene 9d, a crude material was purified by Brindle's aldehyde-removal method ${ }^{[11]}$. After separation operation and removal of solvent, obtained crude residue was dissolved in $\mathrm{MeOH}(1 \mathrm{~mL})$, and saturated aqueous $\mathrm{NaHSO}_{3}(1 \mathrm{~mL})$ was added. After stirring for a few minutes, $\mathrm{MeOH}$ was removed 9d under reduced pressure. The resulting residue was added $\mathrm{H}_{2} \mathrm{O}(2 \sim 3 \mathrm{~mL})$ and extracted with $\mathrm{CH}_{2} \mathrm{Cl}_{2}$. The combined organic layer was dried over $\mathrm{MgSO}_{4}$, filtered and concentrated. The resulting residue was purified through a short pad of silica gel ( $n$-hexane/EtOAc $=30: 1)$ to afford $\mathbf{9 d}$.

According to the modified Procedure $C$ described above, phosphonate (1E,3E)-5 (38.2 mg, 0.100 mmol) was converted to $9 \mathrm{~d}$ (33.0 mg, $79.8 \mu \mathrm{mol}, 80 \%)$, and (1E,3Z)-5 (38.2 mg, $0.100 \mathrm{mmol})$ was converted to $9 d(33.3 \mathrm{mg}, 80.6 \mu \mathrm{mol}, 81 \%)$ by using a $1.0 \mathrm{M}$ solution of KHMDS in THF (110 $\mu \mathrm{L}$, $0.110 \mathrm{mmol})$ and 2-bromobenzaldehyde $(12.8 \mu \mathrm{L}, 0.110 \mathrm{mmol})$.

9d: pale yellow crystal. Mp $138-140{ }^{\circ} \mathrm{C}$ ( $n$-hexane/EtOAc). ${ }^{1} \mathrm{H}$ NMR $\left(500 \mathrm{MHz}, \mathrm{CDCl}_{3}\right) \delta 7.62(\mathrm{~d}$, $J=8.1 \mathrm{~Hz}, 1 \mathrm{H}), 7.43(\mathrm{~d}, J=8.1 \mathrm{~Hz}, 1 \mathrm{H}), 7.29(\mathrm{~d}, J=7.7 \mathrm{~Hz}, 1 \mathrm{H}), 7.24-7.08(\mathrm{~m}, 11 \mathrm{H}), 6.72(\mathrm{~s}, 1 \mathrm{H})$, $6.46(\mathrm{~d}, J=12.1 \mathrm{~Hz}, 1 \mathrm{H}), 6.43(\mathrm{~d}, J=12.1 \mathrm{~Hz}, 1 \mathrm{H}), 5.91(\mathrm{dd}, J=11.5,5.8 \mathrm{~Hz}, 1 \mathrm{H}), 5.83(\mathrm{dd}, J=$ 12.0, $5.2 \mathrm{~Hz}, 1 \mathrm{H}), 4.00(\mathrm{dd}, J=6.9,5.8 \mathrm{~Hz}, 1 \mathrm{H}), 3.97(\mathrm{dd}, J=6.9,5.2 \mathrm{~Hz}, 1 \mathrm{H}) .{ }^{13} \mathrm{C} \mathrm{NMR}(126 \mathrm{MHz}$, $\left.\mathrm{CDCl}_{3}\right) \delta 143.9,137.0,136.9,135.0,134.5,133.0,132.7,132.4,132.1,128.8,128.5(2 \mathrm{C}), 128.3$ (2C), 128.11 (2C), 128.07 (2C), 127.1, 126.8, 126.1 (overlapped, 2C), 124.7, 53.0, 52.7 (One signal could not be judged due to overlap to the signals among 126.8-135.0 ppm.). IR (ATR) $\vee 3060,1490$, 1452, 1022, $697 \mathrm{~cm}^{-1}$. HRMS (FD) $\mathrm{m} / z$ : [M] $]^{+}$calcd for $\mathrm{C}_{26} \mathrm{H}_{21} \mathrm{Br} 412.0827$; found: 412.0808 .

Sample preparation for crystallographic analysis: 9d was dissolved in minimum amount of EtOAc, and the solvent was slowly evaporated under the ambient atmosphere to give a crystalline solid.

The ORTEP drawing of 9d (Ellipsoids are drawn at 50\% probability.)

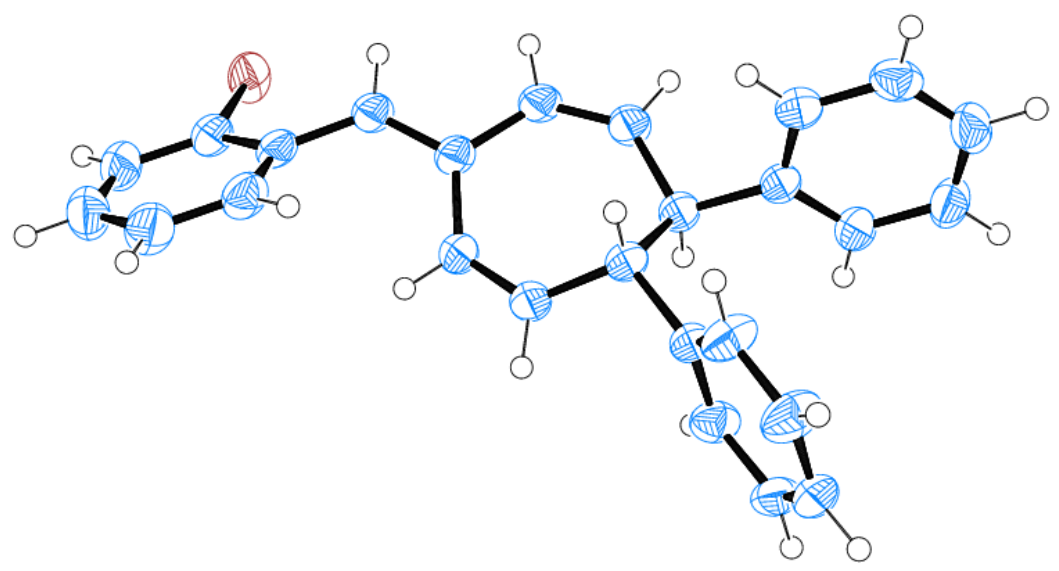


Crystal Data for 9d (CCDC No. 2121233), $\mathrm{C}_{26} \mathrm{H}_{21} \mathrm{Br}(M=413.34 \mathrm{~g} / \mathrm{mol})$, size $0.745 \times 0.485 \times 0.081$ $\mathrm{mm}^{3}$, triclinic, space group P-1 (no. 2), $a=8.4129(5) \AA, b=9.1770(5) \AA, c=14.5231(4) \AA, \alpha=$ $94.903(4)^{\circ}, \beta=102.152(4)^{\circ}, \gamma=113.531(6)^{\circ}, V=986.85(9) \AA^{3}, Z=2, T=150 \mathrm{~K}, \mu(\mathrm{Cu} \mathrm{K \alpha})=0.700$ $\mathrm{mm}^{-1}$, Dcalc $=1.391 \mathrm{~g} / \mathrm{cm}^{3}, 11150$ reflections measured $\left(6.340^{\circ} \leq 2 \theta \leq 155.430^{\circ}\right), 4013$ unique $\left(R_{\text {int }}\right.$ $\left.=0.0450, \mathrm{R}_{\text {sigma }}=0.0405\right)$ which were used in all calculations. The final $R_{1}$ was $0.0604(\mathrm{I}>2 \sigma(\mathrm{I}))$ and $w R_{2}$ was 0.1717 (all data).

\section{Anti-6,7-diphenyl-3-propylidene-1,4-cycloheptadiene (9e)}

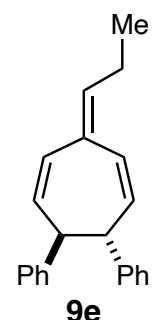

According to Procedure C, phosphonate (1E,3E)-5 (38.2 mg, $0.100 \mathrm{mmol})$ was converted to cycloheptatriene $9 \mathrm{e}(13.3 \mathrm{mg}, 46.4 \mu \mathrm{mol}, 46 \%)$, and (1E,3Z)-5 (38.2 $\mathrm{mg}, 0.100 \mathrm{mmol})$ was converted to $9 \mathrm{e}(12.5 \mathrm{mg}, 43.6 \mu \mathrm{mol}, 44 \%)$ by using a $1.0 \mathrm{M}$ solution of KHMDS in THF $(110 \mu \mathrm{L}, 0.110 \mathrm{mmol})$ and $\mathrm{EtCHO}(10.8 \mu \mathrm{L}, 0.150 \mathrm{mmol})$.

9e: pale yellow oil. ${ }^{1} \mathrm{H}$ NMR $\left(500 \mathrm{MHz}, \mathrm{CDCl}_{3}\right) \delta$ 7.22-7.16 (m, 4H), 7.15-7.08 (m, 6H), $6.54(\mathrm{~d}$, $J=12.1 \mathrm{~Hz}, 1 \mathrm{H}), 6.17(\mathrm{~d}, J=12.0 \mathrm{~Hz}, 1 \mathrm{H}), 5.80(\mathrm{dd}, J=12.1,5.8 \mathrm{~Hz}, 1 \mathrm{H}), 5.72(\mathrm{t}, J=7.7 \mathrm{~Hz}, 1 \mathrm{H})$, $5.59(\mathrm{dd}, J=12.0,5.7 \mathrm{~Hz}, 1 \mathrm{H}), 3.93(\mathrm{dd}, J=6.3,5.8 \mathrm{~Hz}, 1 \mathrm{H}), 3.88(\mathrm{dd}, J=6.3,5.7 \mathrm{~Hz}, 1 \mathrm{H}), 2.34$ $(\mathrm{qd}, J=7.8,7.7 \mathrm{~Hz}, 2 \mathrm{H}), 1.10(\mathrm{t}, J=7.8 \mathrm{~Hz}, 3 \mathrm{H}) .{ }^{13} \mathrm{C} \mathrm{NMR}\left(126 \mathrm{MHz}, \mathrm{CDCl}_{3}\right) \delta 144.5,144.3$, 140.9, 134.7, 133.1, 132.3, 129.4, 128.5 (2C), 128.4 (2C), 128.02 (2C), 127.99 (2C), 126.00, 125.98 125.6, 53.5, 52.9, 21.5, 14.1. IR (ATR) v 3025, 1600, 1491, 1452, $696 \mathrm{~cm}^{-1}$. HRMS (FD) $\mathrm{m} / \mathrm{z}:[\mathrm{M}]^{+}$ calcd for $\mathrm{C}_{22} \mathrm{H}_{22} 286.1722$; found: 286.1735 .

\section{Anti-3-(2-methylpropylidene)-6,7-diphenyl-1,4-cycloheptadiene (9f)}

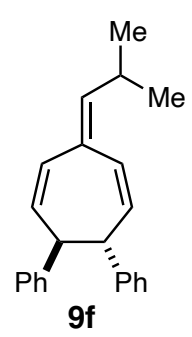

According to Procedure C, phosphonate (1E,3E)-5 (53.1 mg, $0.139 \mathrm{mmol})$ was converted to cycloheptatriene 9 f $(24.0 \mathrm{mg}, 79.9 \mu \mathrm{mol}, 57 \%)$ by using a $1.0 \mathrm{M}$ solution of KHMDS in THF $(153 \mu \mathrm{L}, 0.153 \mathrm{mmol})$ and $i$-PrCHO $(19.0 \mu \mathrm{L}, 0.208 \mathrm{mmol})$. Also, (1E,3Z)-5 (38.2 mg, $0.100 \mathrm{mmol})$ was converted to $9 \mathbf{f}(17.9 \mathrm{mg}, 59.6 \mu \mathrm{mol}, 60 \%)$ by using a $1.0 \mathrm{M}$ solution of KHMDS in THF $(110 \mu \mathrm{L}, 0.110 \mathrm{mmol})$ and $i$-PrCHO (13.7 $\mu \mathrm{L}, 0.150 \mathrm{mmol})$.

9f: pale yellow oil. ${ }^{1} \mathrm{H}$ NMR (500 MHz, $\left.\mathrm{CDCl}_{3}\right) \delta$ 7.21-7.15 (m, 4H), 7.15-7.07 (m, 6H), $6.55(\mathrm{~d}, J$ $=12.6 \mathrm{~Hz}, 1 \mathrm{H}), 6.13(\mathrm{~d}, J=12.1 \mathrm{~Hz}, 1 \mathrm{H}), 5.80(\mathrm{dd}, J=12.6,5.7 \mathrm{~Hz}, 1 \mathrm{H}), 5.59(\mathrm{dd}, J=12.1,5.2$ $\mathrm{Hz}, 1 \mathrm{H}), 5.54(\mathrm{~d}, J=9.2 \mathrm{~Hz}, 1 \mathrm{H}), 3.92(\mathrm{dd}, J=6.3,5.7 \mathrm{~Hz}, 1 \mathrm{H}), 3.87(\mathrm{dd}, J=6.3,5.2 \mathrm{~Hz}, 1 \mathrm{H}), 2.86$ (dqq, $J=9.2,6.3,5.8 \mathrm{~Hz}, 1 \mathrm{H}), 1.09(\mathrm{~d}, J=5.8 \mathrm{~Hz}, 3 \mathrm{H}), 1.08(\mathrm{~d}, J=6.3 \mathrm{~Hz}, 3 \mathrm{H}) .{ }^{13} \mathrm{C}$ NMR $(126$ $\left.\mathrm{MHz}, \mathrm{CDCl}_{3}\right) \delta 146.5,144.6,144.4,133.1,132.5,129.7,128.5$ (2C), 128.4 (overlapped, 2C + 1C), 128.00 (2C), 127.97 (2C), 125.97, 125.95, 125.8, 53.4, 52.9, 27.2, 22.98, 22.96. IR (ATR) v 2958, 1599, 1491, 1452, $696 \mathrm{~cm}^{-1}$. HRMS (FD) $\mathrm{m} / z$ : [M] ${ }^{+}$calcd for $\mathrm{C}_{23} \mathrm{H}_{24} 300.1878$; found: 300.1883 . 


\section{Reactions with Phosphonate Substrates Bearing Various Substituents (Figure 2)}

\section{3-Benzylidene-1-methyl-6-phenyl-1,4-cycloheptadiene (11a)}

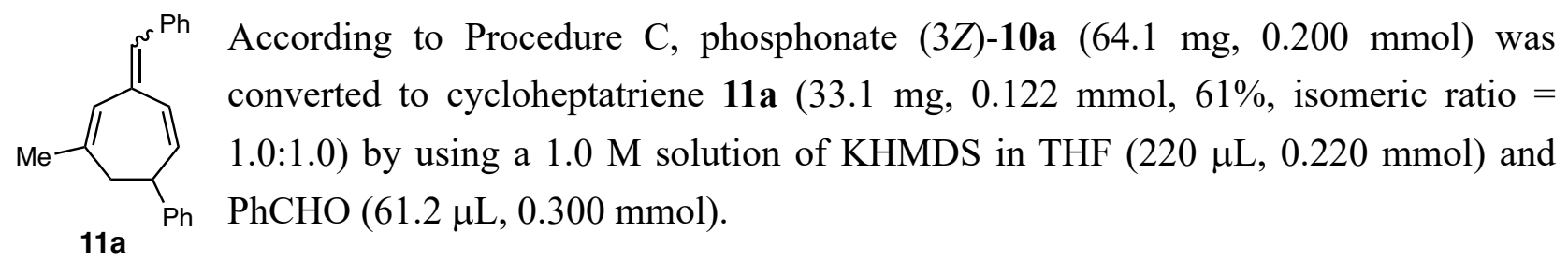

11a (1.0:1.0 isomeric mixture): yellow oil. ${ }^{1} \mathrm{H} \mathrm{NMR}\left(500 \mathrm{MHz}, \mathrm{CDCl}_{3}\right) \delta 7.39-7.20(\mathrm{~m}, 10 \mathrm{H}), 6.53-$ $6.48(\mathrm{~m}, 0.50 \times 3 \mathrm{H}), 6.29(\mathrm{~s}, 0.50 \mathrm{H}), 6.25(\mathrm{~d}, J=11.5 \mathrm{~Hz}, 0.50 \mathrm{H}), 6.10(\mathrm{~s}, 0.50 \mathrm{H}), 5.97(\mathrm{dd}, J=$ $11.5,4.6 \mathrm{~Hz}, 0.50 \mathrm{H}), 5.82(\mathrm{dd}, J=11.5,4.6 \mathrm{~Hz}, 0.50 \mathrm{H}), 3.90-3.84(\mathrm{~m}, 0.50 \mathrm{H}), 3.80-3.74(\mathrm{~m}, 0.50 \mathrm{H})$, $2.72-2.64(\mathrm{~m}, 0.50 \times 2 \mathrm{H}), 2.60(\mathrm{dd}, J=14.3,2.3 \mathrm{~Hz}, 0.50 \mathrm{H}), 2.46(\mathrm{dd}, J=15.5,2.3 \mathrm{~Hz}, 0.50 \mathrm{H})$, $1.80(\mathrm{~s}, 0.50 \times 3 \mathrm{H}), 1.70(\mathrm{~s}, 0.50 \times 3 \mathrm{H}) .{ }^{13} \mathrm{C} \mathrm{NMR}\left(126 \mathrm{MHz}, \mathrm{CDCl}_{3}\right) \delta 145.7,145.0,139.9,138.1$, 137.6, 136.6, 136.0, 135.8, 133.9, 133.0, 132.8, 132.6, 129.82, 129.75, 129.5, 128.5, 128.34, 128.27, $128.02,128.00,127.7,127.4,126.9,126.8,126.6,126.3,126.2,123.6,44.7,44.0,41.7,41.6,27.8$, 27.3 (Only the detected signals were recorded.). IR (ATR) v 2925, 1719, 1598, 1491, 1450, 1272, $1028,695 \mathrm{~cm}^{-1}$. HRMS (FD) $\mathrm{m} / z:[\mathrm{M}]^{+}$calcd for $\mathrm{C}_{21} \mathrm{H}_{20} 272.1565$; found: 272.1568 .

\section{(2-(4-Benzylidene-7-phenylcyclohepta-2,5-dien-1-yl)ethoxy)(tert-butyl)dimethylsilane (11b)}

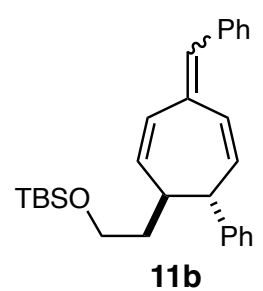

According to Procedure C, phosphonate $(3 E)-\mathbf{1 0 b}(85.0 \mathrm{mg}, 0.183 \mathrm{mmol})$ was converted to cycloheptatriene $11 \mathrm{~b}(57.5 \mathrm{mg}, 0.138 \mathrm{mmol}, 75 \%$, isomeric ratio $=1.0: 1.2)$ by using a $1.0 \mathrm{M}$ solution of KHMDS in THF $(201 \mu \mathrm{L}, 0.201 \mathrm{mmol})$ and PhCHO (28.0 $\mu \mathrm{L}, 0.274 \mathrm{mmol})$. Also, (3Z)-10b (92.8 mg, $0.200 \mathrm{mmol})$ was converted to $11 \mathrm{~b}(71.5 \mathrm{mg}, 0.172 \mathrm{mmol}, 86 \%$, isomeric ratio $=1.0: 1.2 \mathrm{in}$ random order) by using a $1.0 \mathrm{M}$ solution of KHMDS in THF $(220 \mu \mathrm{L}, 0.220 \mathrm{mmol})$ and PhCHO $(30.6 \mu \mathrm{L}$, $0.300 \mathrm{mmol})$.

$11 \mathrm{~b}$ (1.0:1.2 isomeric mixture): yellow oil. ${ }^{1} \mathrm{H}$ NMR (500 MHz, $\left.\mathrm{CDCl}_{3}\right) \delta 7.38-7.16(\mathrm{~m}, 10 \mathrm{H}), 6.69$ $(\mathrm{d}, J=12.1 \mathrm{~Hz}, 0.46 \mathrm{H}), 6.60(\mathrm{~s}, 0.54 \mathrm{H}), 6.58(\mathrm{~s}, 0.46 \mathrm{H}), 6.47(\mathrm{~d}, J=12.1 \mathrm{~Hz}, 0.54 \mathrm{H}), 6.32(\mathrm{~d}, J=$ $12.1 \mathrm{~Hz}, 0.54 \mathrm{H}), 6.17(\mathrm{~d}, J=12.1 \mathrm{~Hz}, 0.46 \mathrm{H}), 5.89(\mathrm{dd}, J=12.1,6.9 \mathrm{~Hz}, 0.46 \mathrm{H}), 5.78-5.70(\mathrm{~m}$, $0.54 \times 2 \mathrm{H}), 5.63(\mathrm{dd}, J=12.1,6.9 \mathrm{~Hz}, 0.46 \mathrm{H}), 3.78-3.65(\mathrm{~m}, 3 \mathrm{H}), 2.88-2.80(\mathrm{~m}, 1 \mathrm{H}), 1.89-1.80$ $(\mathrm{m}, 2 \mathrm{H}), 0.90-0.88(\mathrm{~m}, 9 \mathrm{H}), 0.05-0.02(\mathrm{~m}, 6 \mathrm{H}) .{ }^{13} \mathrm{C} \mathrm{NMR}\left(126 \mathrm{MHz}, \mathrm{CDCl}_{3}\right) \delta 144.0,143.9,137.3$, $136.54,136.50,135.1,134.7,134.6,133.3,133.0,132.6,130.8,130.0,129.8,128.6,128.4,128.2$, 128.12 , 128.10, 128.0, 127.5, 127.2, 127.1, 127.0, 126.2, 61.3, 61.1, 50.0, 49.7, 42.12, 42.05, 38.8, $38.4,26.0,18.3,-5.26,-5.28,-5.32$ (Only the detected signals were recorded.). IR (ATR) $\vee 2952$, 1598, 1491, 1254, 1096, 833, $696 \mathrm{~cm}^{-1}$. HRMS (FD) $\mathrm{m} / \mathrm{z}:[\mathrm{M}]^{+}$calcd for $\mathrm{C}_{28} \mathrm{H}_{36} \mathrm{OSi} 416.2535$; found: 416.2523 . 


\section{3-Benzylidene-6,6-dimethyl-7-phenyl-1,4-cycloheptadiene (11c)}

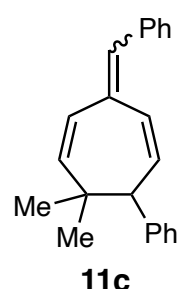

According to Procedure C, phosphonate (3E)-10c (147 mg, $0.440 \mathrm{mmol}$ ) was converted to cycloheptatriene $11 \mathrm{c}(90.1 \mathrm{mg}, 0.315 \mathrm{mmol}, 72 \%$, isomeric ratio $=1.0: 1.1)$ by using a 1.0 M solution of KHMDS in THF ( $484 \mu \mathrm{L}, 0.484 \mathrm{mmol})$ and $\mathrm{PhCHO}(67.3 \mu \mathrm{L}, 0.659$ $\mathrm{mmol})$. Also, (3Z)-10c (102 mg, $0.305 \mathrm{mmol})$ was converted to 11c $(71.5 \mathrm{mg}, 0.250$ mmol, $82 \%$, isomeric ratio $=1.0: 1.2$ ) by using a $1.0 \mathrm{M}$ solution of KHMDS in THF (336 $\mu \mathrm{L}, 0.336 \mathrm{mmol})$ and $\mathrm{PhCHO}(46.7 \mu \mathrm{L}, 0.458 \mathrm{mmol})$.

$11 \mathrm{c}\left(1.0: 1.1\right.$ isomeric mixture): colorless oil. ${ }^{1} \mathrm{H}$ NMR $\left(500 \mathrm{MHz}, \mathrm{CDCl}_{3}\right) \delta 7.40-7.18(\mathrm{~m}, 10 \mathrm{H})$, $6.62-6.56(\mathrm{~m}, 0.47 \mathrm{H}+0.53 \times 2 \mathrm{H}), 6.44(\mathrm{~d}, J=12.0 \mathrm{~Hz}, 0.47 \mathrm{H}), 6.24(\mathrm{~d}, J=12.0 \mathrm{~Hz}, 0.47 \mathrm{H}), 6.10$ $(\mathrm{d}, J=12.0 \mathrm{~Hz}, 0.53 \mathrm{H}), 5.97$ (ddd, $J=12.0,6.9,1.2 \mathrm{~Hz}, 0.53 \mathrm{H}), 5.81(\mathrm{dd}, J=12.0,6.9 \mathrm{~Hz}, 0.47 \mathrm{H})$, $5.58(\mathrm{~d}, J=12.0 \mathrm{~Hz}, 0.47 \mathrm{H}), 5.51(\mathrm{~d}, J=6.9 \mathrm{~Hz}, 0.53 \mathrm{H}), 3.65(\mathrm{~d}, J=6.9 \mathrm{~Hz}, 0.53 \mathrm{H}), 3.48(\mathrm{~d}, J=$ $6.9 \mathrm{~Hz}, 0.47 \mathrm{H}), 1.27(\mathrm{~s}, 0.47 \times 3 \mathrm{H}), 1.08(\mathrm{~s}, 0.53 \times 3 \mathrm{H}), 1.02(\mathrm{~s}, 0.53 \times 3 \mathrm{H}), 0.99(\mathrm{~s}, 0.47 \times 3 \mathrm{H}) .{ }^{13} \mathrm{C}$ NMR $\left(126 \mathrm{MHz}, \mathrm{CDCl}_{3}\right) \delta 142.7,142.4,141.8,140.6,137.3,137.2,136.1,135.8,135.0,134.5$, 132.0, 131.1, 130.1, 130.0, 129.9, 129.80, 129.79, 128.1, 128.0, 127.8, 127.6, 127.2, 127.1, 126.37, $126.35,126.0,125.2,56.9,56.4,39.8,39.4,31.3,30.7,28.8,27.0$ (Only the detected signals were recorded.). IR (ATR) $v 2956,1597,1490,1451,695 \mathrm{~cm}^{-1}$. HRMS (FD) $\mathrm{m} / z$ : [M] $]^{+}$calcd for $\mathrm{C}_{22} \mathrm{H}_{22}$ 286.1722; found: 286.1720 .

\section{7-Benzylidene-4-phenyl-1,2,3,3a,4,7-hexahydroazulene (11d)}

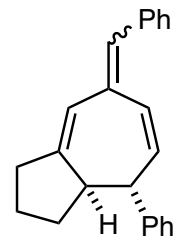

According to Procedure C, phosphonate (3Z)-10d (34.6 mg, $0.100 \mathrm{mmol}$ ) was converted to cycloheptatriene $11 \mathrm{~d}(24.7 \mathrm{mg}, 82.8 \mu \mathrm{mol}, 83 \%$, isomeric ratio $=1.0: 1.3)$ by using a 1.0 M solution of KHMDS in THF $(110 \mu \mathrm{L}, 0.110 \mathrm{mmol})$ and PhCHO $(15.3 \mu \mathrm{L}, 0.150$ $11 \mathrm{~d}$

11d (1.0:1.3 isomeric mixture): colorless oil. ${ }^{1} \mathrm{H}$ NMR $\left(500 \mathrm{MHz}, \mathrm{CDCl}_{3}\right) \delta 7.28-7.07(\mathrm{~m}, 10 \mathrm{H})$, 6.45-6.35 (m, 2H), 6.10 (s, 0.44H), 6.04 (br d, $J=12.1 \mathrm{~Hz}, 0.56 \mathrm{H}), 5.77$ (ddd, $J=12.1,3.5,1.2 \mathrm{~Hz}$, $0.44 \mathrm{H}), 5.61(\mathrm{dd}, J=12.1,2.9 \mathrm{~Hz}, 0.56 \mathrm{H}), 3.41$ (ddd, $J=10.9,3.5,3.5 \mathrm{~Hz}, 0.44 \mathrm{H}), 3.31$ (ddd, $J=$ $10.3,3.5,2.9 \mathrm{~Hz}, 0.56 \mathrm{H}), 2.99-2.91(\mathrm{~m}, 0.56 \mathrm{H}), 2.86-2.78(\mathrm{~m}, 0.44 \mathrm{H}), 2.49-2.45(\mathrm{~m}, 0.44 \times 2 \mathrm{H})$, $2.44-2.35(\mathrm{~m}, 0.56 \times 2 \mathrm{H}), 1.60-1.52(\mathrm{~m}, 1 \mathrm{H}), 1.40-1.25(\mathrm{~m}, 2 \mathrm{H}), 1.18-1.02(\mathrm{~m}, 1 \mathrm{H}) .{ }^{13} \mathrm{C} \mathrm{NMR}(126$ $\left.\mathrm{MHz}, \mathrm{CDCl}_{3}\right) \delta 150.99,150.95,149.0,145.8,145.7,137.74,137.65,137.6,135.4,135.2$, 135.1, $132.7,132.4,131.8,129.71,129.69,128.5,128.04,128.01,127.9,126.7,126.2,125.8,124.2,118.1$, 50.6, 50.4, 49.3, 48.7, 36.5, 36.1, 34.6, 34.4, 24.9, 24.2 (Only the detected signals were recorded.). IR (ATR) v 2953, 1596, 1491, 1450, $696 \mathrm{~cm}^{-1}$. HRMS (FD) $\mathrm{m} / z$ : [M] $]^{+}$calcd for $\mathrm{C}_{23} \mathrm{H}_{22} 298.1722$; found: 298.1711 . 


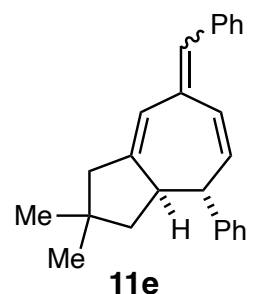

According to Procedure C, phosphonate (3Z)-10e (136 mg, $0.363 \mathrm{mmol}$ ) was converted to cycloheptatriene $11 \mathrm{e}(101 \mathrm{mg}, 0.309 \mathrm{mmol}, 85 \%$, isomeric ratio $=$ 1.0:1.3) by using a $1.0 \mathrm{M}$ solution of KHMDS in THF (399 $\mu \mathrm{L}, 0.399 \mathrm{mmol})$ and PhCHO (55.6 $\mu \mathrm{L}, 0.545 \mathrm{mmol})$.

11e (1.0:1.3 isomeric mixture): pale yellow viscous oil. ${ }^{1} \mathrm{H}-\mathrm{NMR}\left(500 \mathrm{MHz}, \mathrm{CDCl}_{3}\right) \delta 7.37-7.30$ $(\mathrm{m}, 6 \mathrm{H}), 7.25-7.17(\mathrm{~m}, 4 \mathrm{H}), 6.52-6.46(\mathrm{~m}, 2 \mathrm{H}), 6.18-6.11(\mathrm{~m}, 1 \mathrm{H}), 5.84(\mathrm{dd}, J=13.8,3.5 \mathrm{~Hz}$, $0.44 \mathrm{H}), 5.69(\mathrm{dd}, J=14.9,2.9 \mathrm{~Hz}, 0.56 \mathrm{H}), 3.50$ (ddd, $J=10.9,3.5,2.9 \mathrm{~Hz}, 0.44 \mathrm{H}), 3.41$ (ddd, $J=$ $10.3,2.9,2.6 \mathrm{~Hz}, 0.56 \mathrm{H}), 3.29-3.15(\mathrm{~m}, 1 \mathrm{H}), 2.47-2.38(\mathrm{~m}, 1 \mathrm{H}), 2.30(\mathrm{~d}, J=16.0 \mathrm{~Hz}, 0.44 \mathrm{H}), 2.22$ $(\mathrm{d}, J=16.1 \mathrm{~Hz}, 0.56 \mathrm{H}), 1.17(\mathrm{~d}, J=9.2 \mathrm{~Hz}, 0.56 \times 2 \mathrm{H}), 1.14(\mathrm{~d}, J=9.7 \mathrm{~Hz}, 0.44 \times 2 \mathrm{H}), 1.00$ (s, $0.56 \times 3 \mathrm{H}), 1.00(\mathrm{~s}, 0.44 \times 3 \mathrm{H}), 0.92(\mathrm{~s}, 0.56 \times 3 \mathrm{H}), 0.91(\mathrm{~s}, 0.44 \times 3 \mathrm{H}) .{ }^{13} \mathrm{C} \mathrm{NMR}(126 \mathrm{MHz}$ $\left.\mathrm{CDCl}_{3}\right) \delta 150.9,149.0,145.6,137.8,137.7,137.6,135.3,135.2,132.8,132.6,131.7,129.73,129.69$, $128.5,128.4,128.1,128.03,127.98,126.8,126.7,126.2,125.4,124.9,119.1,50.9,50.7,50.6,48.03$, 47.97, 47.9, 47.5, 37.1, 36.6, 28.9, 28.6, 27.1, 26.6 (Only the detected signals were recorded.) IR $\left(\right.$ ATR) $v 2951,1598,1492,1453,699 \mathrm{~cm}^{-1}$. HRMS (FD) $\mathrm{m} / z$ : $[\mathrm{M}]^{+}$calcd for $\mathrm{C}_{25} \mathrm{H}_{26} 326.2035$; found: 326.2042 .

\section{8-Benzylidene-5-phenyl-2,3,4,4a,5,8-hexahydro-1H-benzo[7]annulene (11f)}

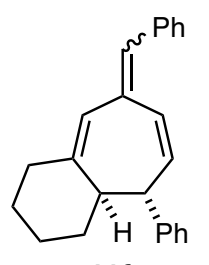

$11 f$

According to Procedure C, phosphonate (3Z)-10f (72.1 mg, $0.200 \mathrm{mmol})$ was converted to cycloheptatriene $11 \mathrm{f}(52.2 \mathrm{mg}, 0.167 \mathrm{mmol}, 84 \%$, isomeric ratio $=$ $1.0: 1.0)$ by using a $1.0 \mathrm{M}$ solution of KHMDS in THF $(220 \mu \mathrm{L}, 0.220 \mathrm{mmol})$ and PhCHO (30.6 $\mu \mathrm{L}, 0.300 \mathrm{mmol})$.

$11 f\left(1.0: 1.0\right.$ isomeric mixture): pale yellow oil. ${ }^{1} \mathrm{H}$ NMR $\left(500 \mathrm{MHz}, \mathrm{CDCl}_{3}\right) \delta 7.42-7.39(\mathrm{~m}, 1 \mathrm{H})$, $7.38-7.33(\mathrm{~m}, 2 \mathrm{H}), 7.33-7.29(\mathrm{~m}, 2 \mathrm{H}), 7.29-7.19(\mathrm{~m}, 5 \mathrm{H}), 6.51(\mathrm{~s}, 0.50 \mathrm{H}), 6.44(\mathrm{~s}, 0.50 \mathrm{H}), 6.41(\mathrm{~d}$, $J=12.1 \mathrm{~Hz}, 0.50 \mathrm{H}), 6.33-6.27(\mathrm{~m}, 1 \mathrm{H}), 6.14(\mathrm{~s}, 0.50 \mathrm{H}), 5.95(\mathrm{ddd}, J=12.1,6.3,1.2 \mathrm{~Hz}, 0.50 \mathrm{H})$, $5.78(\mathrm{dd}, J=11.5,5.2 \mathrm{~Hz}, 0.50 \mathrm{H}), 3.64(\mathrm{ddd}, J=10.9,6.3,1.2 \mathrm{~Hz}, 0.50 \mathrm{H}), 3.57$ (ddd, $J=10.9,5.2$, $1.2 \mathrm{~Hz}, 0.50 \mathrm{H}), 2.70-2.63(\mathrm{~m}, 0.50 \mathrm{H}), 2.55-2.47(\mathrm{~m}, 0.50 \mathrm{H}), 2.37-2.27(\mathrm{~m}, 1 \mathrm{H}), 2.18-2.09(\mathrm{~m}, 1 \mathrm{H})$, $1.81-1.71(\mathrm{~m}, 1 \mathrm{H}), 1.71-1.63(\mathrm{~m}, 1 \mathrm{H}), 1.49-1.24(\mathrm{~m}, 3 \mathrm{H}), 1.20(\mathrm{dddd}, J=11.5,11.5,11.5,3.5 \mathrm{~Hz}$, $0.50 \mathrm{H}), 1.08$ (dddd, $J=11.5,11.5,11.5,3.5 \mathrm{~Hz}, 0.50 \mathrm{H}) .{ }^{13} \mathrm{C} \mathrm{NMR}\left(126 \mathrm{MHz}, \mathrm{CDCl}_{3}\right) \delta 146.9$, 145.20, 145.16, 144.9, 137.6, 137.5, 136.10, 136.07, 136.0, 134.3, 133.8, 132.4, 131.8, 129.8, 129.7, $129.6,128.5,128.4,128.2,128.14,128.07,128.0,126.9,126.2,126.1,125.7,120.8,50.7,50.0,47.8$, $46.3,40.2,38.4,34.6,33.5,28.2,27.4,26.1,25.0$ (Only the detected signals were recorded.). IR $\left(\right.$ ATR) $\vee 2925,1596,1491,1444,694 \mathrm{~cm}^{-1}$. HRMS (FD) $\mathrm{m} / z$ : $[\mathrm{M}]^{+}$calcd for $\mathrm{C}_{24} \mathrm{H}_{24} 312.1878$; found: 312.1876 . 


\section{Reactions of Trienes with Alkyl groups at Each Terminus (Scheme 4)}

\section{3-Benzylidene-6,7-anti-dicyclohexyl-1,4-cycloheptadiene (13a)}

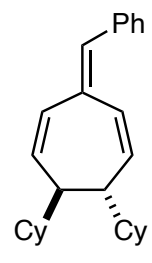

According to Procedure C, phosphonate (3E)-12a (39.5 mg, $0.100 \mathrm{mmol})$ was converted to cycloheptatriene 13a (25.1 mg, $72.4 \mu \mathrm{mol}, 72 \%)$, and (3Z)-12a (39.5 mg, $0.100 \mathrm{mmol}$ ) was converted to $\mathbf{1 3 a}(30.8 \mathrm{mg}, 88.9 \mu \mathrm{mol}, 89 \%)$ by using a $1.0 \mathrm{M}$ solution of KHMDS in THF $(110 \mu \mathrm{L}, 0.110 \mathrm{mmol})$ and PhCHO $(15.3 \mu \mathrm{L}, 0.150 \mathrm{mmol})$.

13a

13a: pale yellow oil. ${ }^{1} \mathrm{H}$ NMR $\left(500 \mathrm{MHz}, \mathrm{CDCl}_{3}\right) \delta 7.35-7.29(\mathrm{~m}, 4 \mathrm{H}), 7.23(\mathrm{t}, J=7.7 \mathrm{~Hz}, 1 \mathrm{H})$, $6.47(\mathrm{~s}, 1 \mathrm{H}), 6.46(\mathrm{~d}, J=11.5 \mathrm{~Hz}, 1 \mathrm{H}), 6.08(\mathrm{~d}, J=11.5 \mathrm{~Hz}, 1 \mathrm{H}), 5.97(\mathrm{dd}, J=11.5,8.1 \mathrm{~Hz}, 1 \mathrm{H})$, $5.82(\mathrm{dd}, J=11.5,8.6 \mathrm{~Hz}, 1 \mathrm{H}), 2.17-2.12(\mathrm{~m}, 2 \mathrm{H}), 1.97-1.88(\mathrm{~m}, 2 \mathrm{H}), 1.82-1.76(\mathrm{~m}, 2 \mathrm{H}), 1.71-$ $1.64(\mathrm{~m}, 4 \mathrm{H}), 1.64-1.57(\mathrm{~m}, 3 \mathrm{H}), 1.48-1.39(\mathrm{~m}, 1 \mathrm{H}), 1.23-1.08(\mathrm{~m}, 6 \mathrm{H}), 1.02-0.91(\mathrm{~m}, 2 \mathrm{H}), 0.87-$ $0.76(\mathrm{~m}, 2 \mathrm{H}) .{ }^{13} \mathrm{C} \mathrm{NMR}\left(126 \mathrm{MHz}, \mathrm{CDCl}_{3}\right) \delta 137.6,137.5,135.6,133.5,133.1,131.4,129.8(2 \mathrm{C})$, 128.0, 126.8, 125.5 (2C), 45.9, 45.8, 42.4, 41.4, 32.3, 32.1, 31.03, 30.97, 26.74, 26.70, 26.43, 26.35, 26.2 (overlapped, 2C). IR (ATR) $v 2918,1446,694 \mathrm{~cm}^{-1}$. HRMS (FD) $\mathrm{m} / z$ : [M] $]^{+}$calcd for $\mathrm{C}_{26} \mathrm{H}_{34}$ 346.2661; found: 346.2672 .

\section{3-Benzilidene-1,4-cycloheptadiene 13b}

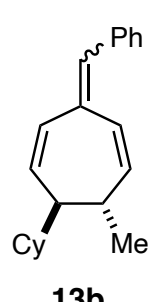

According to Procedure C, phosphonate (3Z)-12b (65.3 mg, $0.200 \mathrm{mmol})$ was converted to cycloheptatriene $\mathbf{1 3 b}(32.0 \mathrm{mg}, 0.115 \mathrm{mmol}, 57 \%$, isomeric ratio $=1.0: 1.0)$ by using a 1.0 M solution of KHMDS in THF $(210 \mu \mathrm{L}, 0.210 \mathrm{mmol})$. (NOTE: As same as the reaction of $(3 E)-\mathbf{1 2 b}$, the generation of tetraene $\mathbf{1 4}$ was also detected in another trial of the reaction of (3Z)-12b, while the yield of the $\mathbf{1 3 b}$ was constant. This fact implied $\mathbf{1 4}$ decomposed partially or completely under the reaction conditions.)

13b (1.0:1.0 isomeric mixture): pale yellow oil. ${ }^{1} \mathrm{H}$ NMR $\left(500 \mathrm{MHz}, \mathrm{CDCl}_{3}\right) \delta 7.36-7.29(\mathrm{~m}, 4 \mathrm{H})$, $7.25-7.20(\mathrm{~m}, 1 \mathrm{H}), 6.49-6.44(\mathrm{~m}, 0.50 \times 3 \mathrm{H}), 6.41(\mathrm{~d}, J=11.5 \mathrm{~Hz}, 0.50 \mathrm{H}), 6.13(\mathrm{~d}, J=12.0 \mathrm{~Hz}$, $0.50 \mathrm{H}), 6.04(\mathrm{~d}, J=12.0 \mathrm{~Hz}, 0.50 \mathrm{H}), 5.95(\mathrm{dd}, J=12.0,8.0 \mathrm{~Hz}, 0.50 \mathrm{H}), 5.89(\mathrm{dd}, J=11.5,7.5 \mathrm{~Hz}$, $0.50 \mathrm{H}), 5.79(\mathrm{dd}, J=12.0,7.5 \mathrm{~Hz}, 0.50 \mathrm{H}), 5.73(\mathrm{dd}, J=12.0,6.9 \mathrm{~Hz}, 0.50 \mathrm{H}), 2.68-2.58(\mathrm{~m}, 1 \mathrm{H})$, 2.04-1.96 (m, 1H), 1.95-1.84 (m, 1H), 1.82-1.58 (m, 4H), 1.51-1.41 (m, 0.50H), 1.30-1.09 (0.50 $\times 5 \mathrm{H}), 1.07(\mathrm{~d}, J=5.9 \mathrm{~Hz}, 0.50 \times 3 \mathrm{H}), 1.04-0.86(0.50 \times 5 \mathrm{H}) .{ }^{13} \mathrm{C} \mathrm{NMR}\left(126 \mathrm{MHz}, \mathrm{CDCl}_{3}\right) \delta$ $137.59,137.56,137.2,137.1,136.6,135.4,134.3,133.5,133.3,132.8,131.5,131.0,129.79,129.76$, $128.0,126.9,126.8,125.9,125.3,51.0,50.9,42.4,41.5,34.24,34.21,31.5,31.3,31.23,31.18,26.72$, 26.68, 26.52, 26.46, 26.4, 21.0, 20.5 (Only the detected signals were recorded.). IR (ATR) $\vee 2921$, $1446,694 \mathrm{~cm}^{-1}$. HRMS (FD) $\mathrm{m} / z:[\mathrm{M}]^{+}$calcd for $\mathrm{C}_{21} \mathrm{H}_{26} 278.2035$; found: 278.2023 . 


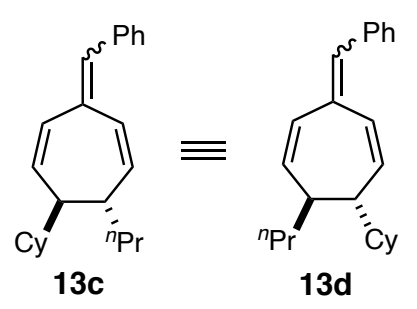

THF (169 $\mu \mathrm{L}, 0.169 \mathrm{mmol})$ and PhCHO $(23.5 \mu \mathrm{L}, 0.230 \mathrm{mmol})$. In the same way, phosphonate (3E)-12d ( $85.8 \mathrm{mg}, 0.242 \mathrm{mmol}$ ) was converted to cycloheptatriene 13d (47.6 mg, $0.155 \mathrm{mmol}$, $64 \%$, isomeric ratio $=1.0: 1.0)$ by using a $1.0 \mathrm{M}$ solution of KHMDS in THF $(266 \mu \mathrm{L}, 0.266 \mathrm{mmol})$ and PhCHO (37.0 $\mu \mathrm{L}, 0.363 \mathrm{mmol})$. Also, (3Z)-12d (58.1 mg, $0.164 \mathrm{mmol})$ was converted to 13d $(27.1 \mathrm{mg}, 88.4 \mu \mathrm{mol}, 54 \%$, isomeric ratio $=1.0: 1.0)$ by using a $1.0 \mathrm{M}$ solution of KHMDS in THF $(180 \mu \mathrm{L}, 0.180 \mathrm{mmol})$ and $\mathrm{PhCHO}(25.1 \mu \mathrm{L}, 0.246 \mathrm{mmol})$.

13c (13d) (1.0:1.0 isomeric mixture): pale yellow oil. ${ }^{1} \mathrm{H}$ NMR $\left(500 \mathrm{MHz}, \mathrm{CDCl}_{3}\right) \delta 7.35-7.28(\mathrm{~m}$, 4H), 7.24-7.20 (m, 1H), 6.47-6.41 (m, 2H), $6.10(\mathrm{~d}, J=12.0 \mathrm{~Hz}, 0.50 \mathrm{H}), 6.06(\mathrm{~d}, J=12.0 \mathrm{~Hz}$, $0.50 \mathrm{H}), 5.98-5.90(\mathrm{~m}, 1 \mathrm{H}), 5.82-5.75(\mathrm{~m}, 1 \mathrm{H}), 2.53-2.46(\mathrm{~m}, 1 \mathrm{H}), 2.08-2.00(\mathrm{~m}, 1 \mathrm{H}), 1.97-1.87$ $(\mathrm{m}, 1 \mathrm{H}), 1.74-1.65(\mathrm{~m}, 1 \mathrm{H}), 1.74-1.64(\mathrm{~m}, 2 \mathrm{H}), 1.62-1.58(\mathrm{~m}, 2 \mathrm{H}), 1.52-0.84(\mathrm{~m}, 12 \mathrm{H}) .{ }^{13} \mathrm{C}$ NMR $\left(126 \mathrm{MHz}, \mathrm{CDCl}_{3}\right) \delta 137.63,137.61,137.4,137.3,135.62,135.58,133.55,133.4,133.2,133.0$, $131.4,131.3,129.8,128.0,126.83,126.81,125.65,125.61,49.2,48.9,42.5,41.5,39.40,39.36,38.2$, $37.5,32.2,31.9,31.19,31.17,26.73,26.69,26.5,26.3,21.0,20.8,14.30,14.29$ (Only the detected signals were recorded.) IR (ATR) $v 2921,1446,694 \mathrm{~cm}^{-1}$. HRMS (FD) $m / z$ : [M] calcd for $\mathrm{C}_{23} \mathrm{H}_{30}$ 306.2348; found: 306.2362 .

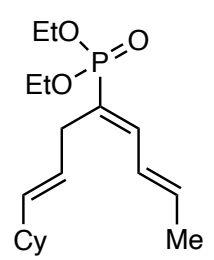

$(3 E)-12 b$

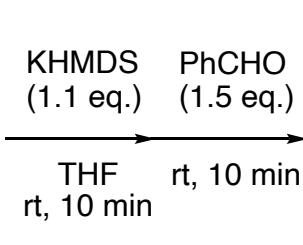

$\underset{\mathrm{rt}, 10 \mathrm{~min}}{\mathrm{THF}} \mathrm{rt} 10 \mathrm{~min}$

According to Procedure C, a mixture of cycloheptatriene 13b and tetraene 14 (27.0 mg, $97.0 \mu \mathrm{mol}$, $48 \%, \mathbf{1 3 b}: \mathbf{1 4}=1.6: 1.0)$ was obtained from $(3 E)-\mathbf{1 2 b}(65.3 \mathrm{mg}, 0.200 \mathrm{mmol})$, a $1.0 \mathrm{M}$ solution of KHMDS in THF $(210 \mu \mathrm{L}, 0.220 \mathrm{mmol})$ and PhCHO $(30.6 \mu \mathrm{L}, 0.300 \mathrm{mmol})$.

The mixture of $\mathbf{1 3 b}$ and $\mathbf{1 4}$ was dissolved in toluene $(0.20 \mathrm{~mL})$, and $N$-phenyl maleimide $(19.4 \mathrm{mg}$, $0.112 \mathrm{mmol}$ ) was added at room temperature. The mixture was heated to $90^{\circ} \mathrm{C}$ and stirred for $11 \mathrm{~h}$. Then, the mixture was cooled to room temperature, and the solvent was removed under reduced pressure. Purification through a short pad of silica gel $(n$-hexane/EtOAc $=30: 1)$ afforded cycloheptatriene $\mathbf{1 3 b}(14.9 \mathrm{mg}, 53.5 \mu \mathrm{mol}, 27 \%$ from $(3 E)-\mathbf{1 2 b}$, isomeric ratio $=1.0: 1.1)$ 
Analytically pure tetraene $\mathbf{1 4}$ was partially separated by PTLC ( $n$-hexane only) as a 1.0:1.1 isomeric mixture.

14 (1.0:1.1 isomeric mixture): pale yellow oil. ${ }^{1} \mathrm{H}$ NMR $\left(500 \mathrm{MHz}, \mathrm{CDCl}_{3}\right) \delta 7.36-7.30(\mathrm{~m}, 3 \mathrm{H})$, $7.28-7.20(\mathrm{~m}, 2 \mathrm{H}), 6.70(\mathrm{~d}, J=15.5 \mathrm{~Hz}, 0.53 \mathrm{H}), 6.60(\mathrm{~s}, 0.47 \mathrm{H}), 6.49-6.33(\mathrm{~m}, 3 \mathrm{H}), 5.52-5.47(\mathrm{~m}$, $2 \mathrm{H}), 5.26(\mathrm{dd}, J=5.2,1.2 \mathrm{~Hz}, 0.53 \mathrm{H}), 5.23(\mathrm{~d}, J=5.2 \mathrm{~Hz}, 0.47 \mathrm{H}), 5.11$ (br s, $0.53 \mathrm{H}), 5.09$ (br s, $0.47 \mathrm{H}), 3.16(\mathrm{~d}, J=4.6 \mathrm{~Hz}, 0.47 \times 2 \mathrm{H}), 3.06(\mathrm{~d}, J=4.0 \mathrm{~Hz}, 0.53 \times 2 \mathrm{H}), 2.02-1.92(\mathrm{~m}, 1 \mathrm{H}), 1.76-$ $1.67(\mathrm{~m}, 4 \mathrm{H}), 1.67-1.60(\mathrm{~m}, 1 \mathrm{H}), 1.32-1.02(\mathrm{~m}, 5 \mathrm{H}) .{ }^{13} \mathrm{C} \mathrm{NMR}\left(126 \mathrm{MHz}, \mathrm{CDCl}_{3}\right) \delta 138.7,138.3$, $138.2,137.8,137.7,137.60,137.58,137.3,132.7,131.4,130.8,130.2,129.9,129.8,129.4,128.9$, $128.14,128.07,126.8,126.6,125.1,124.5,117.3,116.8,40.8,40.7,37.4,33.1,33.0,31.6,30.8$, 26.2, 26.1, 26.0. IR (ATR) v 2922, 1447, 906, 732, $697 \mathrm{~cm}^{-1}$. HRMS (FD) $\mathrm{m} / \mathrm{z}$ : [M] $]^{+}$calcd for $\mathrm{C}_{21} \mathrm{H}_{26}$ 278.2035; found: 278.2044 .

\section{Synthesis and Reaction of a Substrate with an Oxygenated Functionality (Scheme 5)}
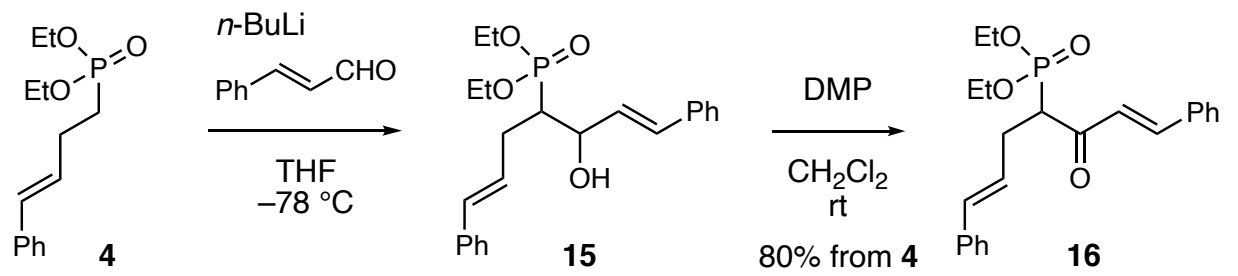

To a solution of phosphonate $4(268 \mathrm{mg}, 1.00 \mathrm{mmol})$ in THF $(5.0 \mathrm{~mL})$ at $-78^{\circ} \mathrm{C}$ was slowly added a $2.69 \mathrm{M}$ solution of $n$-BuLi in $n$-hexane $(372 \mu \mathrm{L}, 1.00 \mathrm{mmol})$. After stirring at $-78{ }^{\circ} \mathrm{C}$ for $0.5 \mathrm{~h}$, trans-cinnamaldehyde $(132 \mu \mathrm{L}, 1.05 \mathrm{mmol})$ was added, and the mixture was further stirred for 10 min at $-78{ }^{\circ} \mathrm{C}$. Then, the reaction was quenched by adding saturated aqueous $\mathrm{NH}_{4} \mathrm{Cl}$. EtOAc $(5 \mathrm{~mL})$ was added to the mixture and the two layers were separated, and the aqueous layer was extracted with EtOAc $(2 \times 5 \mathrm{~mL})$. The combined organic layer was dried over $\mathrm{MgSO}_{4}$, filtered, and concentrated to give crude $\mathbf{1 5}$ as a 3:2 mixture of diastereomers.

The obtained crude product was dissolved in $\mathrm{CH}_{2} \mathrm{Cl}_{2}(5.0 \mathrm{~mL})$ and added Dess-Martin periodinane (DMP) (467 mg, $1.10 \mathrm{mmol}$ ) at room temperature, and the resulting mixture was stirred for $15 \mathrm{~min}$. Then the mixture was added saturated aqueous $\mathrm{NH}_{4} \mathrm{Cl}(5 \mathrm{~mL})$ and solid $\mathrm{Na}_{2} \mathrm{~S}_{2} \mathrm{O}_{3}$. After stirring vigorously for several hours, the two layers were separated, and the aqueous layer was extracted with $\mathrm{Et}_{2} \mathrm{O}(3 \times 5 \mathrm{~mL})$. The combined organic layer was dried over $\mathrm{MgSO}_{4}$, filtered, and concentrated under reduced pressure. Purification by flash column chromatography $\left(\mathrm{SiO}_{2}, n\right.$-hexane/EtOAc $=$ 1:1) afforded $\beta$-ketophosphonate 16 (317 $\mathrm{mg}, 0.796 \mathrm{mmol}, 80 \%$ from 4).

16: yellow oil. ${ }^{1} \mathrm{H}$ NMR $\left(500 \mathrm{MHz}, \mathrm{CDCl}_{3}\right) \delta 7.64(\mathrm{~d}, J=15.5 \mathrm{~Hz}, 1 \mathrm{H}), 7.60-7.54(\mathrm{~m}, 2 \mathrm{H}), 7.41-$ $7.36(\mathrm{~m}, 3 \mathrm{H}), 7.30-7.23(\mathrm{~m}, 4 \mathrm{H}), 7.18(\mathrm{t}, J=7.2 \mathrm{~Hz}, 1 \mathrm{H}), 7.00(\mathrm{~d}, J=16.0 \mathrm{~Hz}, 1 \mathrm{H}), 6.46(\mathrm{~d}, J=$ 
$15.5 \mathrm{~Hz}, 1 \mathrm{H}), 6.13(\mathrm{dt}, J=15.5,7.2 \mathrm{~Hz}, 1 \mathrm{H}), 4.20-4.16(\mathrm{~m}, 4 \mathrm{H}), 3.56(\mathrm{ddd}, J=20.4,10.3,4.0 \mathrm{~Hz}$, $1 \mathrm{H}), 3.09-2.99(\mathrm{~m}, 1 \mathrm{H}), 2.84-2.74(\mathrm{~m}, 1 \mathrm{H}), 1.34(\mathrm{t}, J=6.9 \mathrm{~Hz}, 3 \mathrm{H}), 1.32(\mathrm{t}, J=7.5 \mathrm{~Hz}, 3 \mathrm{H}) .{ }^{13} \mathrm{C}$ NMR (126 MHz, $\left.\mathrm{CDCl}_{3}\right) \delta 193.6,143.5,136.9,134.2,132.2,130.7,128.8$ (2C), 128.5 (2C), 128.4 (2C), 127.2, $126.5\left(\mathrm{~d}, J_{\mathrm{C}-\mathrm{P}}=15.6 \mathrm{~Hz}\right), 126.1(2 \mathrm{C}), 125.5,62.69\left(\mathrm{~d}, J_{\mathrm{C}-\mathrm{P}}=7.2 \mathrm{~Hz}\right), 62.67\left(\mathrm{~d}, J_{\mathrm{C}-\mathrm{P}}=\right.$ $6.0 \mathrm{~Hz}), 51.8\left(\mathrm{~d}, J_{\mathrm{C}-\mathrm{P}}=125 \mathrm{~Hz}\right), 29.9\left(\mathrm{~d}, J_{\mathrm{C}-\mathrm{P}}=4.8 \mathrm{~Hz}\right), 16.4\left(\mathrm{~d}, J_{\mathrm{C}-\mathrm{P}}=6.0 \mathrm{~Hz}\right), 16.3\left(\mathrm{~d}, J_{\mathrm{C}-\mathrm{P}}=6.0\right.$ $\mathrm{Hz}) .{ }^{31} \mathrm{P}$ NMR $\left(202 \mathrm{MHz}, \mathrm{CDCl}_{3}\right) \delta 22.5$. IR (ATR) $\vee 2979,1607,1247,1019,961 \mathrm{~cm}^{-1}$. HRMS (FD) $m / z:[\mathrm{M}]^{+}$calcd for $\mathrm{C}_{23} \mathrm{H}_{27} \mathrm{O}_{4} \mathrm{P} 398.1647$; found: 398.1637 .
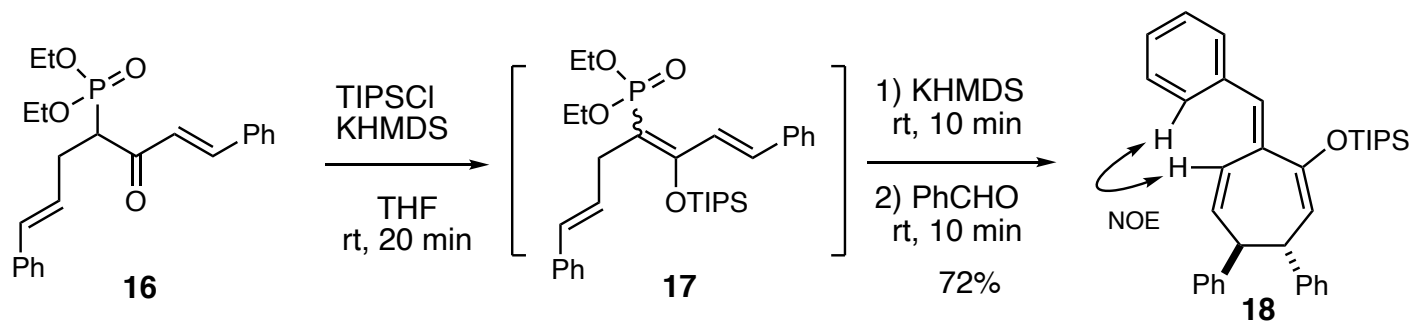

To a solution of $16(79.7 \mathrm{mg}, 0.200 \mathrm{mmol})$ in THF $(2.0 \mathrm{~mL})$ at room temperature was added a 1.0 M solution of KHMDS (210 $\mu \mathrm{L}, 210 \mathrm{mmol})$, and after $4 \mathrm{~min}$, TIPSCl (44.5 $\mu \mathrm{L}, 0.210 \mathrm{mmol})$ was added. After stirring for $25 \mathrm{~min}$ at room temperature, a $1.0 \mathrm{M}$ solution of KHMDS $(210 \mu \mathrm{L}, 210$ mmol) was added. The mixture was further stirred for $10 \mathrm{~min}$, and then added PhCHO $(30.6 \mu \mathrm{L}$, $0.300 \mathrm{mmol}$ ). After stirring for $10 \mathrm{~min}$, the reaction was quenched by addition of saturated aqueous $\mathrm{NaHCO}_{3}(2 \mathrm{~mL})$, and the mixture was diluted with EtOAc $(2 \mathrm{~mL})$. The two layers were separated, and the aqueous layer was extracted with EtOAc $(2 \times 2 \mathrm{~mL})$. The combined organic layer was dried over $\mathrm{MgSO}_{4}$, filtered, and concentrated under reduced pressure. Purification by flash column chromatography $\left(\mathrm{SiO}_{2}, n\right.$-hexane/Et $\left.2 \mathrm{O}=50: 1\right)$ afforded cyclic triene 18 (73.2 mg, $0.144 \mathrm{mmol}$, $72 \%)$.

18: pale yellow oil. ${ }^{1} \mathrm{H}$ NMR $\left(500 \mathrm{MHz}, \mathrm{CDCl}_{3}\right) \delta 7.43(\mathrm{~d}, J=7.5 \mathrm{~Hz}, 2 \mathrm{H}), 7.38(\mathrm{dd}, J=7.7,7.7$ $\mathrm{Hz}, 2 \mathrm{H}), 7.34(\mathrm{~s}, 1 \mathrm{H}), 7.28(\mathrm{t}, J=7.5 \mathrm{~Hz}, 1 \mathrm{H}), 7.16-7.10(\mathrm{~m}, 4 \mathrm{H}), 7.08-7.03(\mathrm{~m}, 6 \mathrm{H}), 6.56(\mathrm{~d}, J=$ $11.5 \mathrm{~Hz}, 1 \mathrm{H}), 5.84$ (ddd, $J=11.5,4.6,1.2 \mathrm{~Hz}, 1 \mathrm{H}), 5.28$ (d, $J=5.8 \mathrm{~Hz}, 1 \mathrm{H}), 3.93$ (dd, $J=8.6,5.2$ $\mathrm{Hz}, 1 \mathrm{H}), 3.90-3.85$ (m, 1H), 1.22 (sept, $J=7.5 \mathrm{~Hz}, 3 \mathrm{H}), 1.12$ (d, $J=7.5 \mathrm{~Hz}, 9 \mathrm{H}), 1.10$ (d, $J=7.5$ $\mathrm{Hz}, 9 \mathrm{H}) .{ }^{13} \mathrm{C} \mathrm{NMR}\left(126 \mathrm{MHz}, \mathrm{CDCl}_{3}\right) \delta 150.5,144.7,143.8,137.0,134.4,134.1,132.3,130.2(2 \mathrm{C})$, 128.4 (2C), 128.2 (2C), 128.1 (2C), 127.94 (2C), 127.92 (2C), 127.4, 125.9, 125.83, 125.77, 112.8, 52.5, 49.5, 18.1 (6C), 12.7 (3C). IR (ATR) v 2943, 1196, 1126, 881, $695 \mathrm{~cm}^{-1}$. HRMS (FD) $m / z$ : $[\mathrm{M}]^{+}$calcd for $\mathrm{C}_{35} \mathrm{H}_{42} \mathrm{OSi}$ 506.3005; found: 506.2996. 

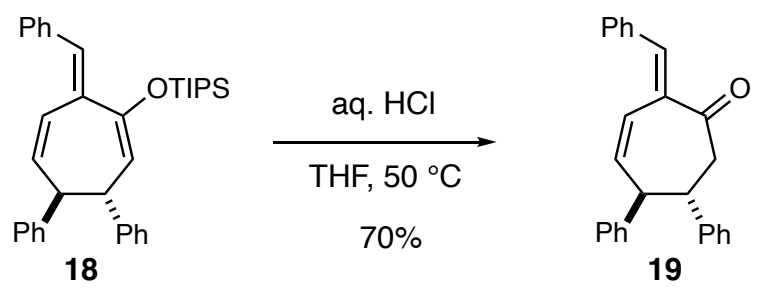

To a solution of silyl enol ether $\mathbf{1 8}(31.7 \mathrm{mg}, 62.5 \mu \mathrm{mol})$ in THF $(0.47 \mathrm{~mL})$ at room temperature was added $1 \mathrm{M}$ aq. $\mathrm{HCl}(0.13 \mathrm{~mL})$. The mixture was warmed to $50^{\circ} \mathrm{C}$ and stirred for $40 \mathrm{~min}$. Then, the mixture was cooled to room temperature, and diluted with EtOAc $(0.5 \mathrm{~mL})$. The two layers were separated, and the aqueous layer was extracted with EtOAc $(2 \times 0.5 \mathrm{~mL})$. The combined organic layer was dried over $\mathrm{MgSO}_{4}$, filtered, and concentrated under reduced pressure. Purification by flash column chromatography $\left(\mathrm{SiO}_{2}, n\right.$-hexane/EtOAc $\left.=20: 1\right)$ and removal of TIPSOH by heating to $90{ }^{\circ} \mathrm{C}$ under high vacuum afforded ketone 19 (15.4 mg, $\left.43.9 \mu \mathrm{mol}, 70 \%\right)$.

19: white solid. Mp 116-118 ${ }^{\circ} \mathrm{C}$ (n-hexane/EtOAc). ${ }^{1} \mathrm{H}$ NMR $\left(500 \mathrm{MHz}, \mathrm{CDCl}_{3}\right) \delta 7.69(\mathrm{~s}, 1 \mathrm{H})$, $7.60(\mathrm{~d}, J=7.5 \mathrm{~Hz}, 2 \mathrm{H}), 7.47-7.36(\mathrm{~m}, 3 \mathrm{H}), 7.19-7.07(\mathrm{~m}, 6 \mathrm{H}), 6.96(\mathrm{~d}, J=6.3 \mathrm{~Hz}, 2 \mathrm{H}), 6.91(\mathrm{~d}, J$ $=6.9 \mathrm{~Hz}, 2 \mathrm{H}), 6.60(\mathrm{~d}, J=12.6 \mathrm{~Hz}, 1 \mathrm{H}), 6.07(\mathrm{dd}, J=12.6,4.0 \mathrm{~Hz}, 1 \mathrm{H}), 3.82(\mathrm{dd}, J=10.9,4.0 \mathrm{~Hz}$, $1 \mathrm{H}), 3.38$ (ddd, $J=10.9,8.6,2.3 \mathrm{~Hz}, 1 \mathrm{H}), 3.29$ (dd, $J=13.8,8.6,1 \mathrm{H}), 3.12$ (d, $J=13.8,2.3 \mathrm{~Hz}$, $1 \mathrm{H}) .{ }^{13} \mathrm{C} \mathrm{NMR}\left(126 \mathrm{MHz}, \mathrm{CDCl}_{3}\right) \delta 200.7,143.9,142.2,138.7,135.7,135.4,134.5,130.9(2 \mathrm{C})$, 129.3, 128.5 (2C), 128.3 (2C), 128.25 (2C), $128.18(2 \mathrm{C}), 127.5$ (2C), 126.6, 126.4, 124.0, 56.4, 48.2, 47.4. IR (ATR) $\vee 3027,1682,1563,1491,695 \mathrm{~cm}^{-1}$. HRMS (FD) $\mathrm{m} / z$ : [M] $]^{+}$calcd for $\mathrm{C}_{26} \mathrm{H}_{22} \mathrm{O}$ 350.1671 ; found: 350.1680 . 


\section{References}

(1) Kato, R.; Saito, H.; Uda, S.; Domon, D.; Ikeuchi, K.; Suzuki, T.; Tanino, K. Synthesis of Seven-Membered Cross-Conjugated Cyclic Trienes by $8 \pi$ Electrocyclic Reaction. Org. Lett. 2021, 23, 8878-8882.

(2) (a) Sato, K.; Tanino, K. Synthetic Studies on Cyclocitrinol: Construction of the ABC Ring System Based on Epoxy-Nitrile Cyclization. Synlett 2021, 32, 674-678. (b) Hazelden, I. R.; Ma, X.; Langer, T.; Bower, J. F. Diverse $N$-Heterocyclic Ring Systems via Aza-Heck Cyclizations of $N$-(Pentafluorobenzoyloxy)sulfonamides. Angew. Chem., Int. Ed. 2016, 55, 11198-11202.

(3) (a) Ando, K.; Yamada, K. Highly $E$-selective solvent-free Horner-Wadsworth-Emmons reaction catalyzed by DBU. Green Chem. 2011, 13, 1143-1146. (b) Xiong, W.; Xu, G.; Tang, W. PChiral Monophosphorus Ligands for Asymmetric Copper-Catalyzed Allylic Alkylation. Organometallics 2019, 38, 4003-4013.

(4) Triantafyllakis, M.; Tofi, M.; Montagnon, T.; Kouridaki, A.; Vassilikogiannakis, G. Org. Lett. 2014, 16, 3150-3153.

(5) Yanai, H.; Takahashi, A.; Taguchi, T. 1,4-Addition of silicon dienoates to $\alpha, \beta$-unsaturated aldehydes catalyzed by in situ-generated silicon Lewis acid. Chem. Commun. 2010, 46, 8728-8730.

(6) Kueh, J. S. H.; Mellor, M.; Pattenden G. Synthetic photochemistry. Elaboration of the tricyclo[6.3.0.0]-undecane ('hirsutane') carbon skeleton by intramolecular photocyclisations of dicyclopent-1-enylmethanes. J. Chem. Soc., Perkin Trans. 1 1981, 1052-1057.

(7) Mancini, I.; Guella, G.; Pietra, F. Synthesis of the Bretonins, Polyolefinic Esterified Glyceryl Ethers of an Unidentified Sponge from the North-Brittany Sea: Absolute Configuration and Novel Structure Assignment. Helv. Chim. Acta 1991, 74, 941-950.

(8) Pachali, S.; Hofmann, C.; Rapp, G.; Schobert, R.; Baro, A.; Frey, W.; Laschat, S. Stereoselective Synthesis of (2E,4Z)-Dienamides Employing (Triphenylphosphoranylidene)ketene. Eur. J. Org. Chem. 2009, 2828-2835.

(9) Zgani, I.; Menut, C.; Seman, M.; Gallois, V.; Laffont, V.; Liautard, J.; Liautard, J.-P.; Criton, M.; Montero J.-L. Synthesis of Prenyl Pyrophosphonates as New Potent Phosphoantigens Inducing Selective Activation of Human V $\gamma 9 \mathrm{~V} \delta 2$ T Lymphocytes. J. Med. Chem. 2004, 47, 4600-4612.

(10) Teulade, M.-P.; Savignac, P.; Aboujaoude, E. E.; Liétge, S.; Collignon, N. Alkylidènediphosphonates et vinylphosphonates: une démarche synthétiques sélective par voie carbanionique. J. Organomet. Chem. 1986, 304, 283-300.

(11) Boucher, M. M.; Furigay, M. H.; Quach, P. K.; Brindle, C. S. Liquid-Liquid Extraction Protocol for the Removal of Aldehydes and Highly Reactive Ketones from Mixtures. Org. Process Res. Dev. 2017, 21, 1394-1403. 
${ }^{1} \mathrm{H},{ }^{13} \mathrm{C}$, and ${ }^{31} \mathrm{P}$ NMR spectra

NMR spectra of $4\left[{ }^{1} \mathrm{H}(500 \mathrm{MHz}),{ }^{13} \mathrm{C}(126 \mathrm{MHz})\right.$, and ${ }^{31} \mathrm{P}(202 \mathrm{MHz})$ in $\left.\mathrm{CDCl}_{3}\right]$
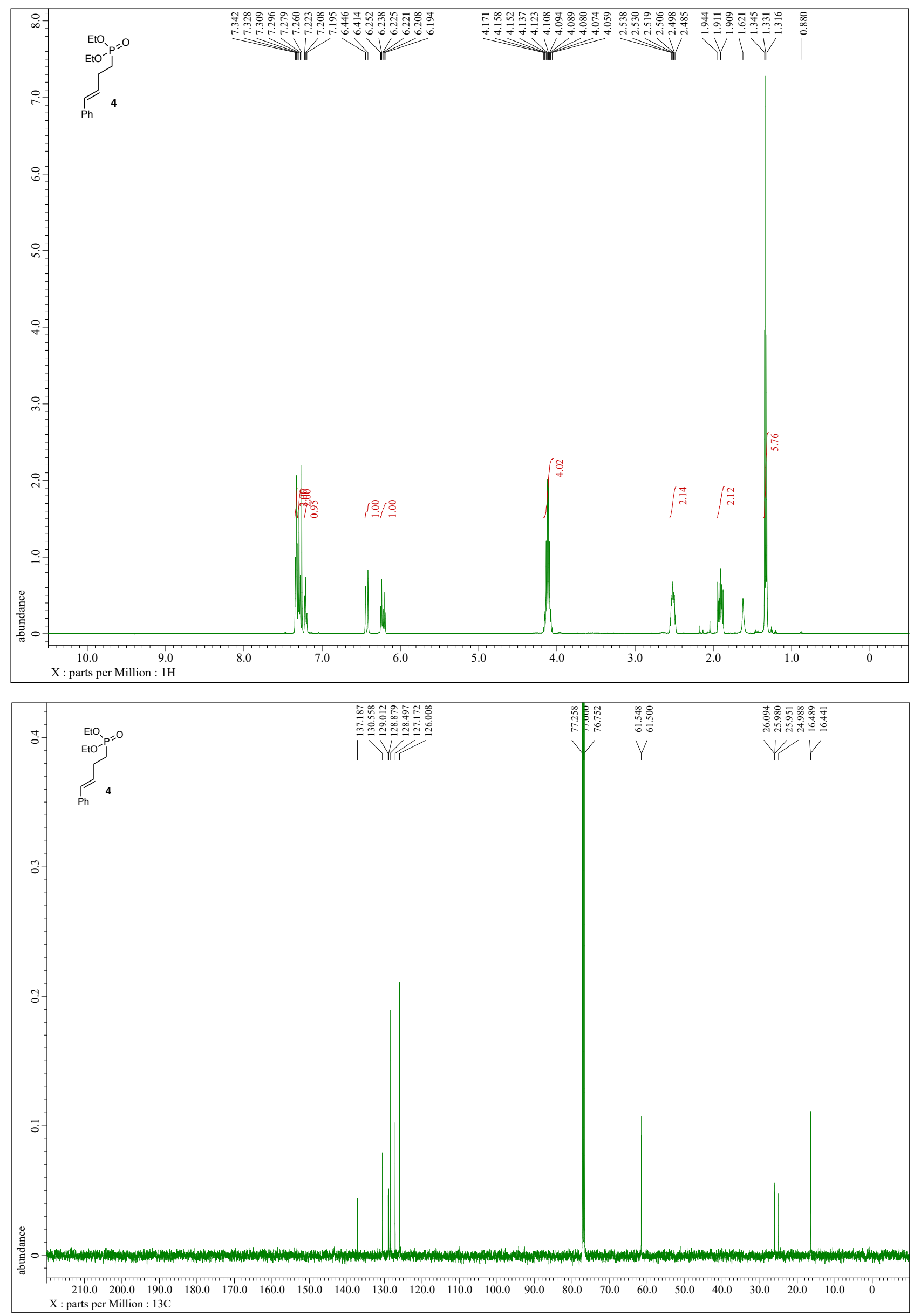


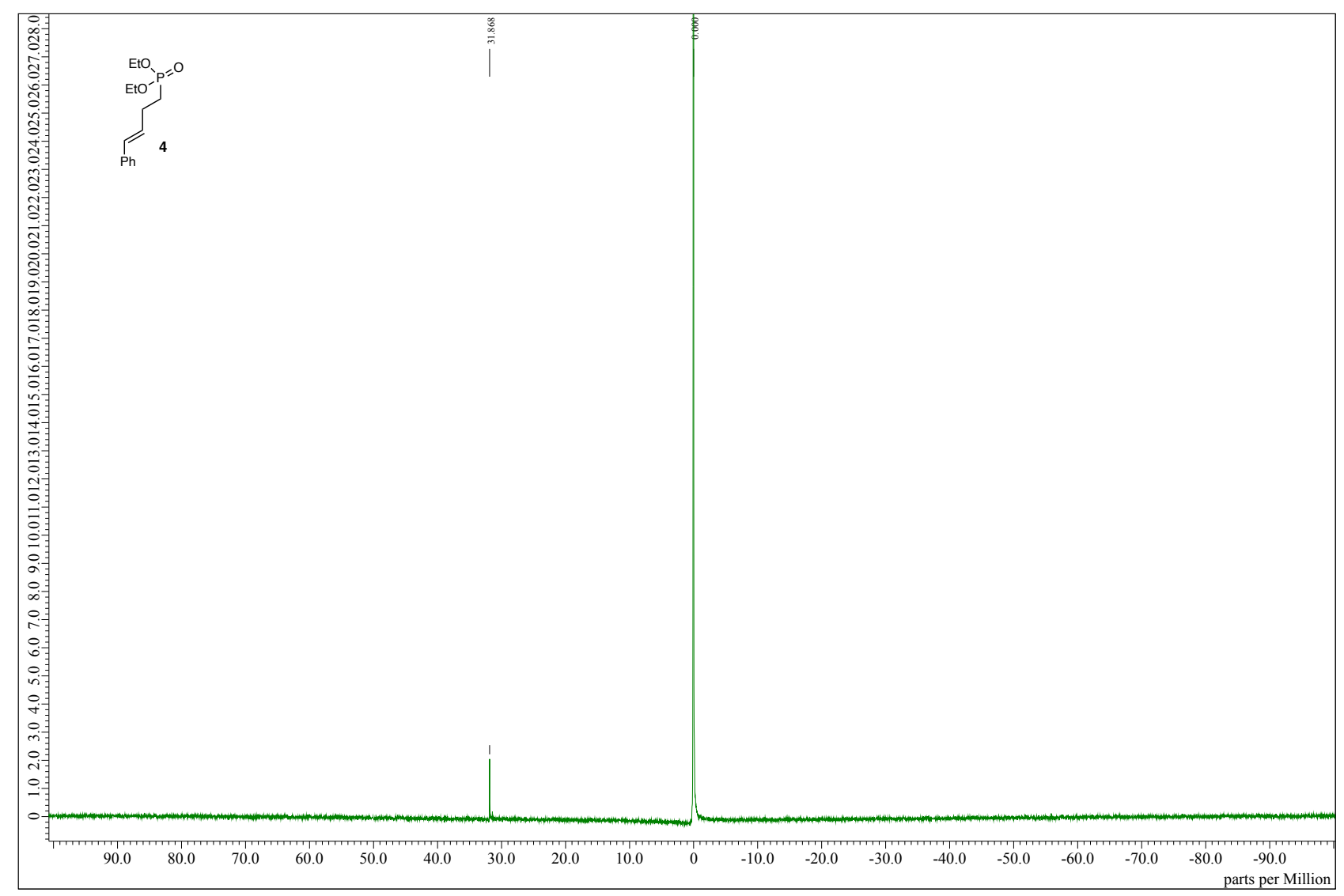


NMR spectra of $\mathbf{4 b}\left[{ }^{1} \mathrm{H}(500 \mathrm{MHz}),{ }^{13} \mathrm{C}(126 \mathrm{MHz})\right.$, and ${ }^{31} \mathrm{P}(202 \mathrm{MHz})$ in $\left.\mathrm{CDCl}_{3}\right]$
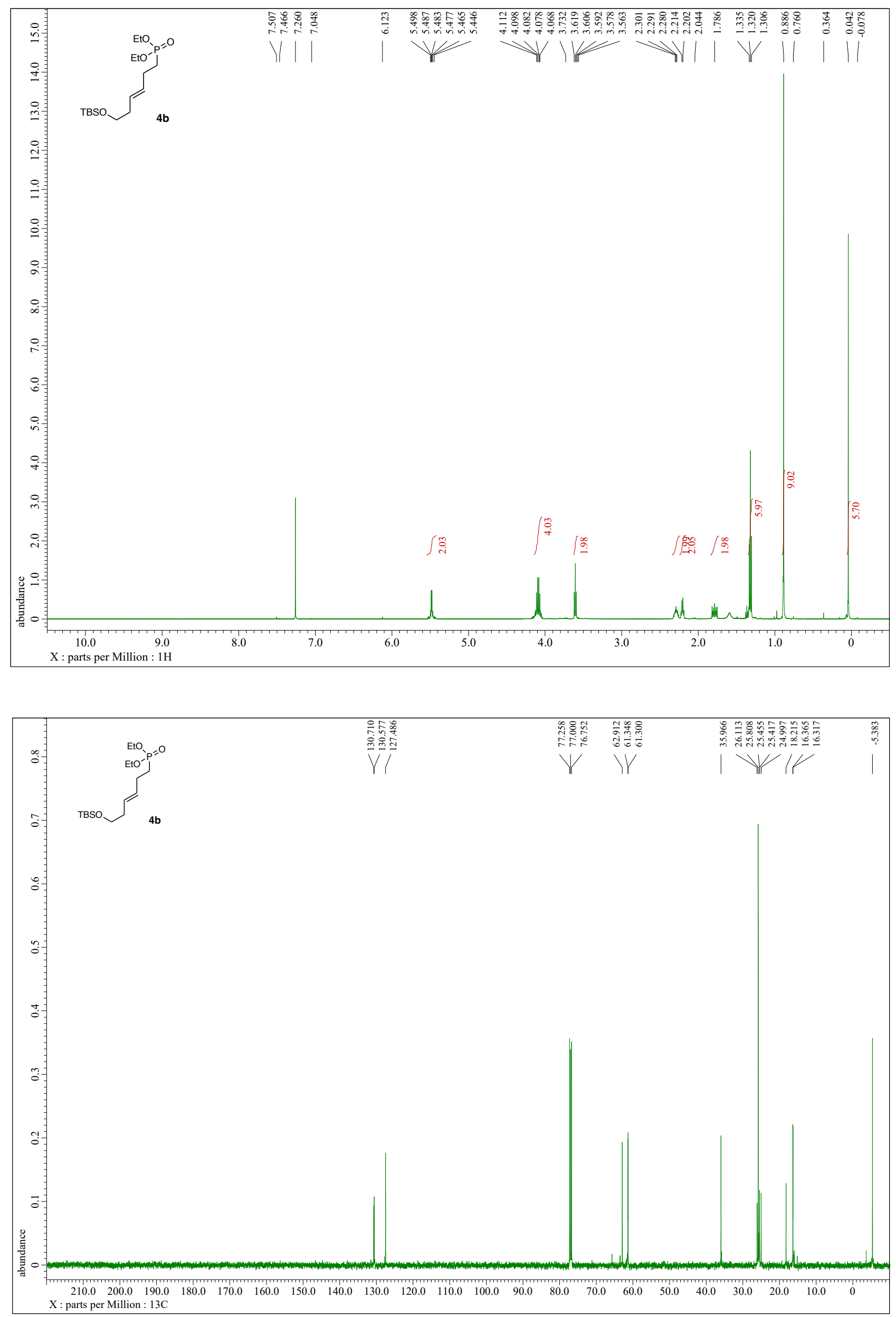


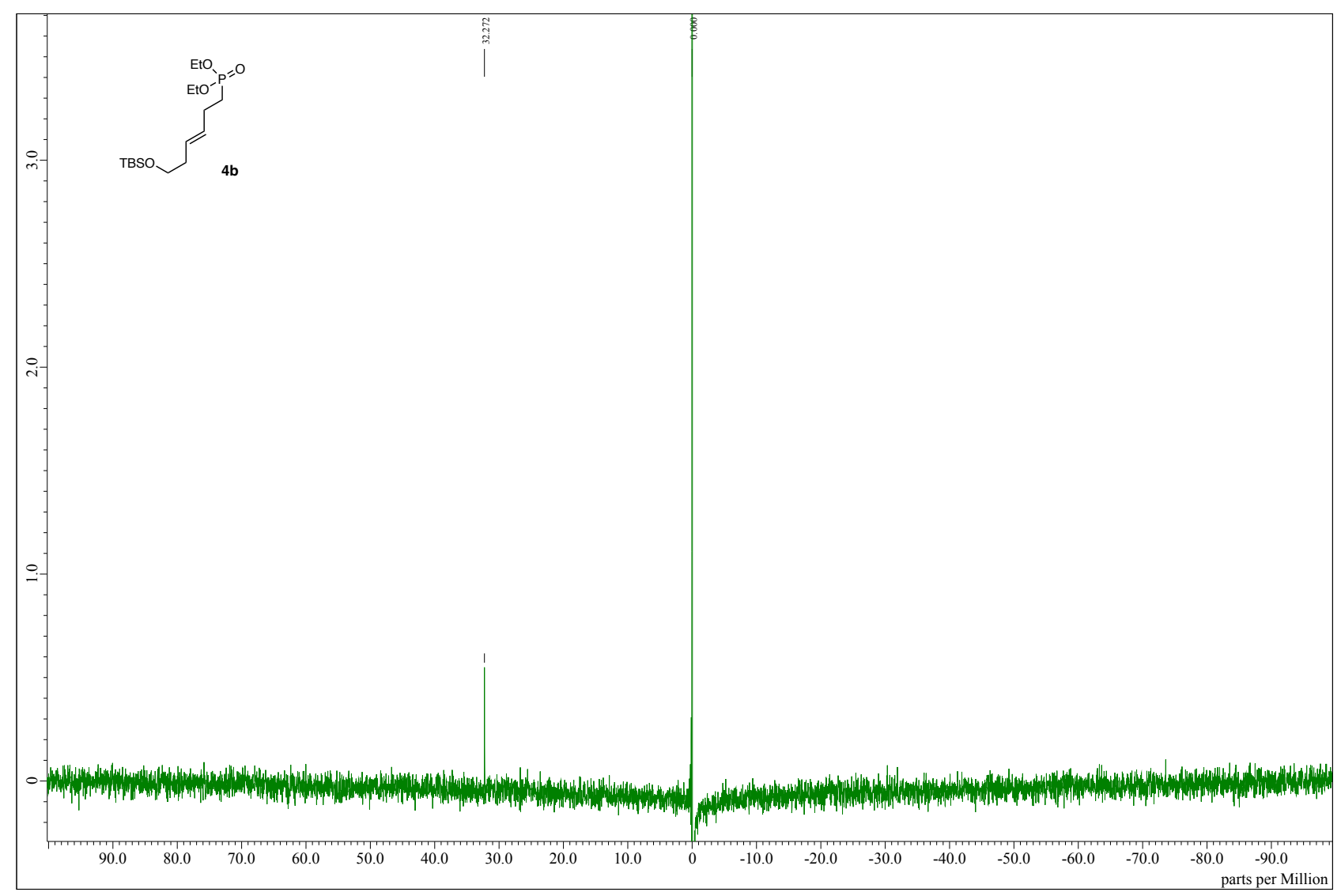


NMR spectra of $4 \mathbf{d}\left[{ }^{1} \mathrm{H}(500 \mathrm{MHz}),{ }^{13} \mathrm{C}(126 \mathrm{MHz})\right.$, and ${ }^{31} \mathrm{P}(202 \mathrm{MHz})$ in $\left.\mathrm{CDCl}_{3}\right]$
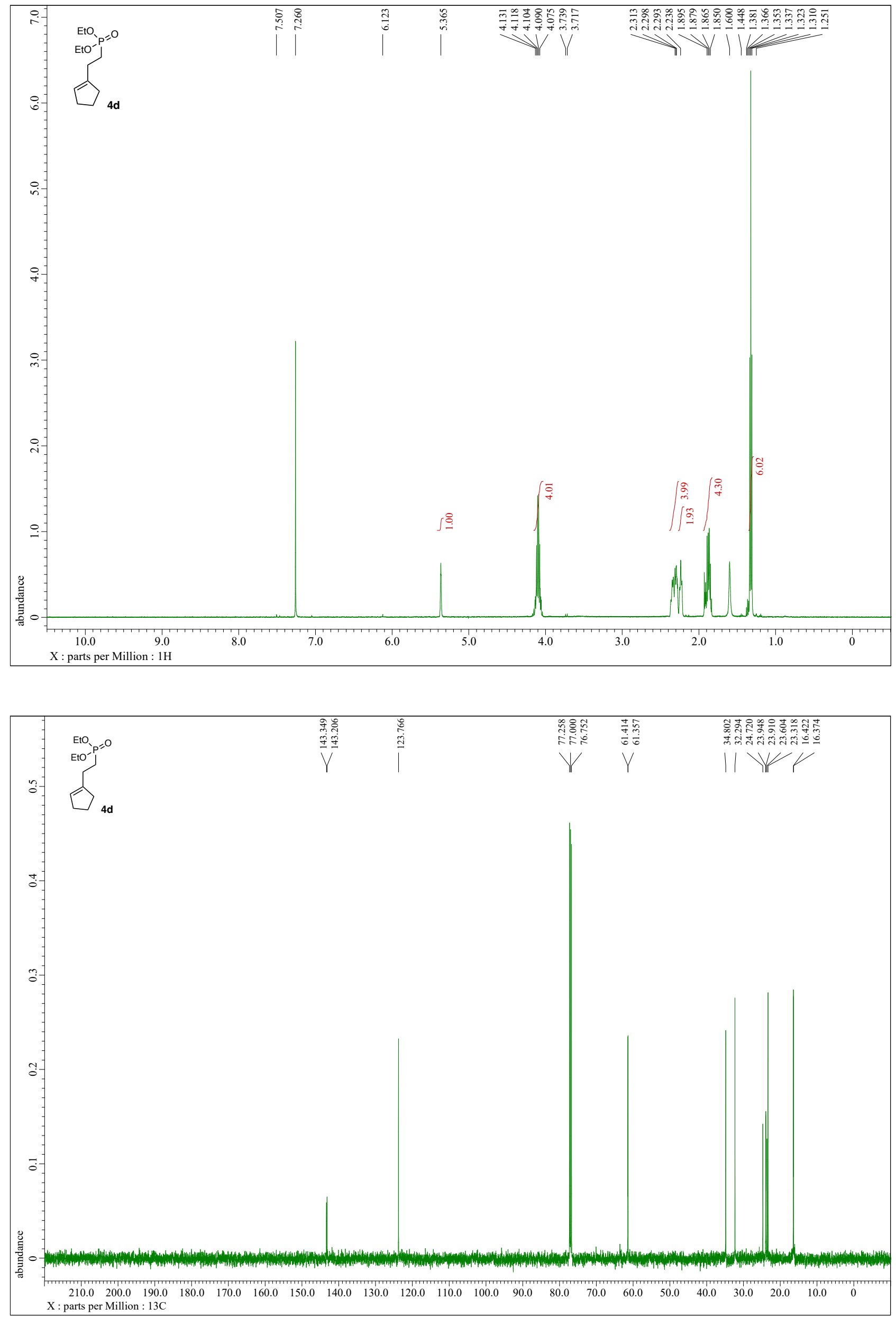


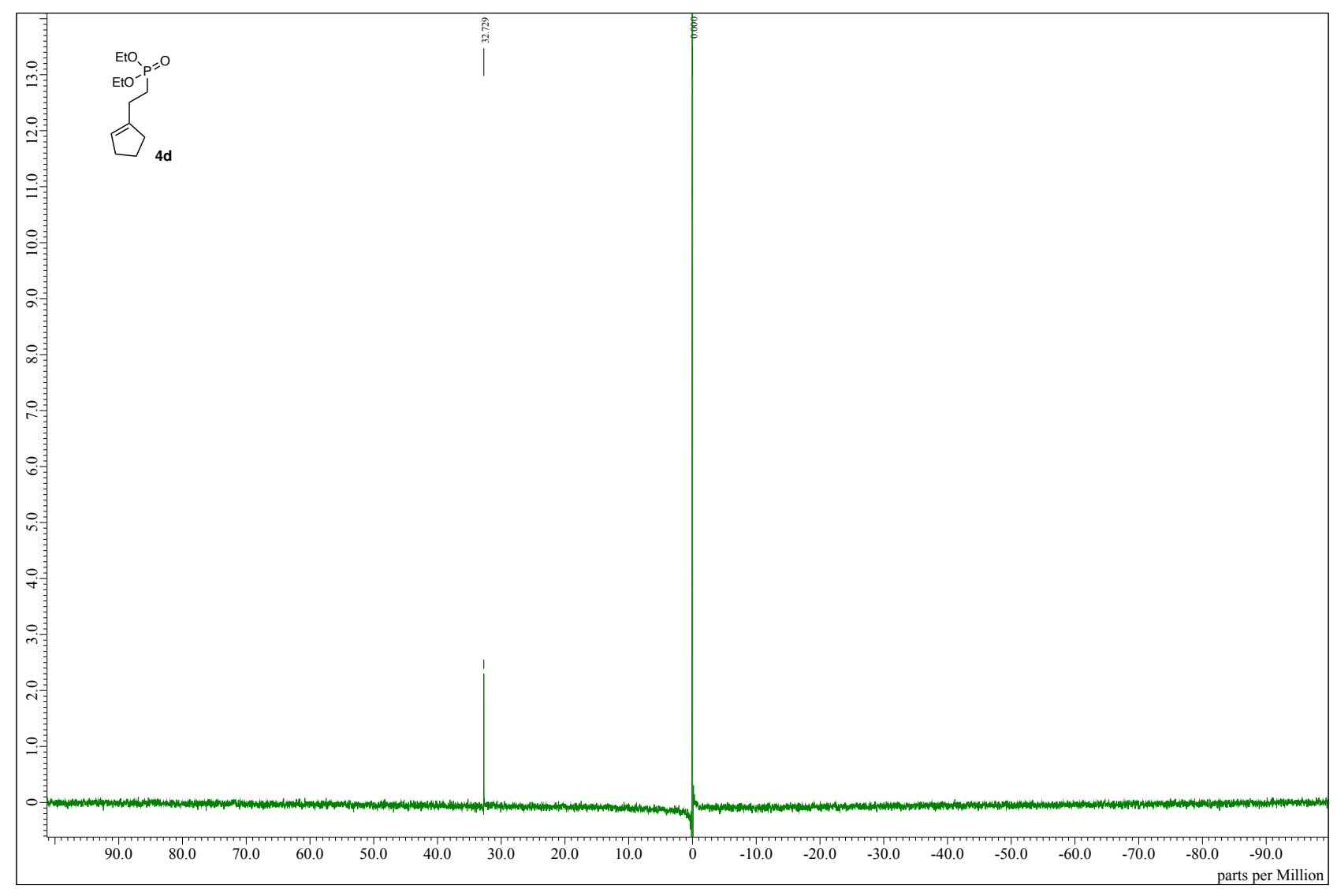


NMR spectra of $4 \mathbf{e}\left[{ }^{1} \mathrm{H}(500 \mathrm{MHz}),{ }^{13} \mathrm{C}(126 \mathrm{MHz})\right.$, and ${ }^{31} \mathrm{P}(202 \mathrm{MHz})$ in $\left.\mathrm{CDCl}_{3}\right]$
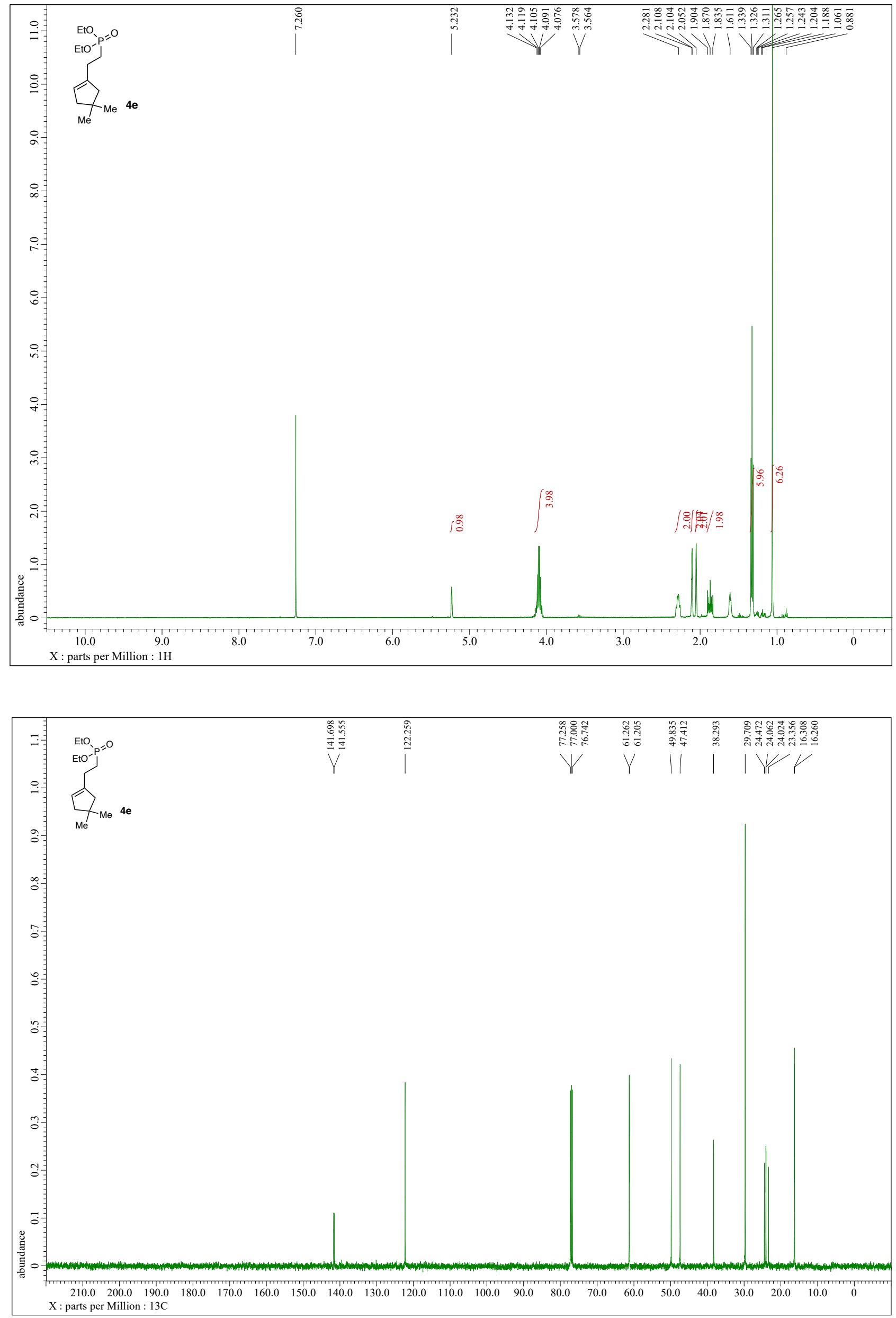


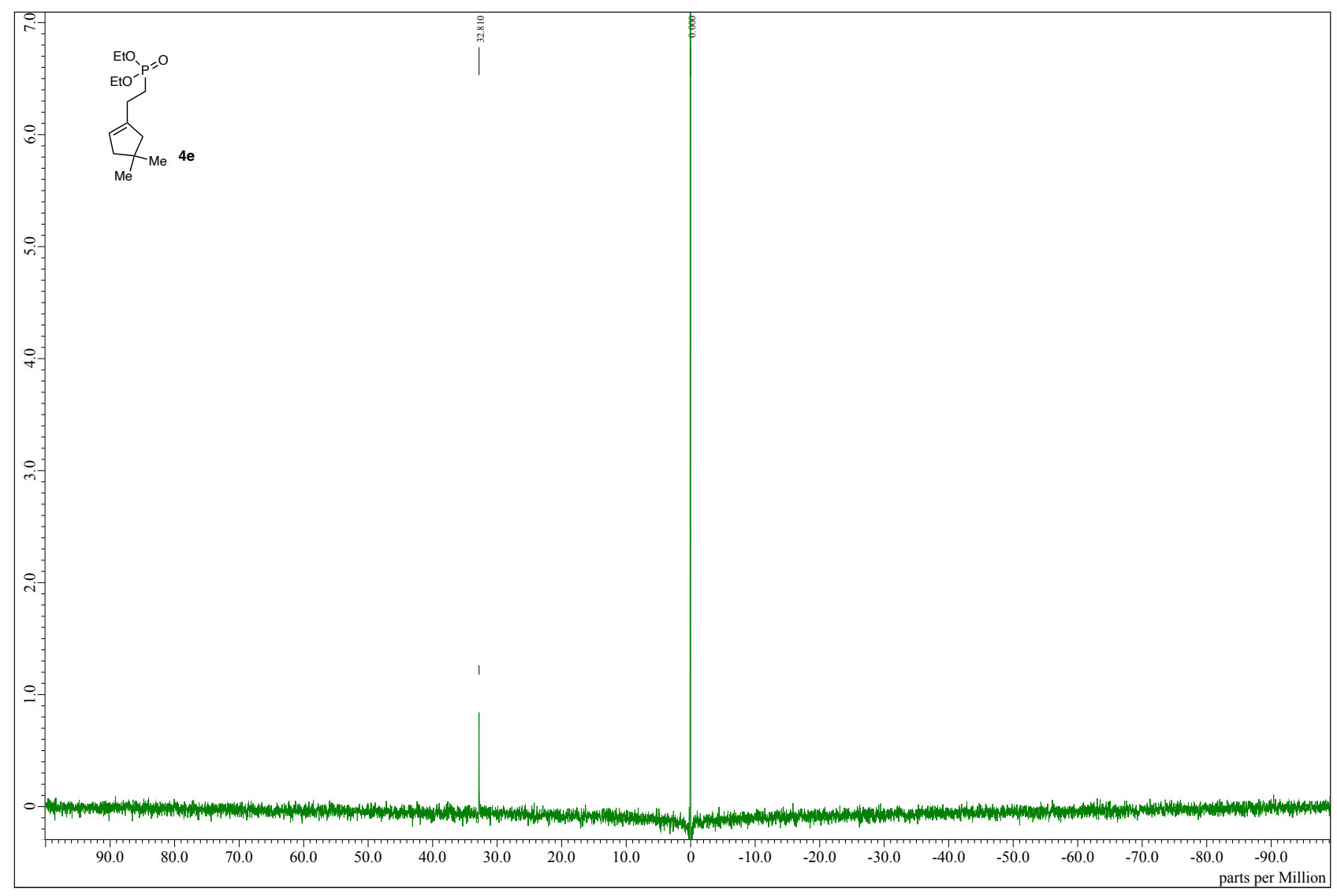


NMR spectra of $4 \mathbf{f}\left[{ }^{1} \mathrm{H}(500 \mathrm{MHz}),{ }^{13} \mathrm{C}(126 \mathrm{MHz})\right.$, and ${ }^{31} \mathrm{P}(202 \mathrm{MHz})$ in $\left.\mathrm{CDCl}_{3}\right]$
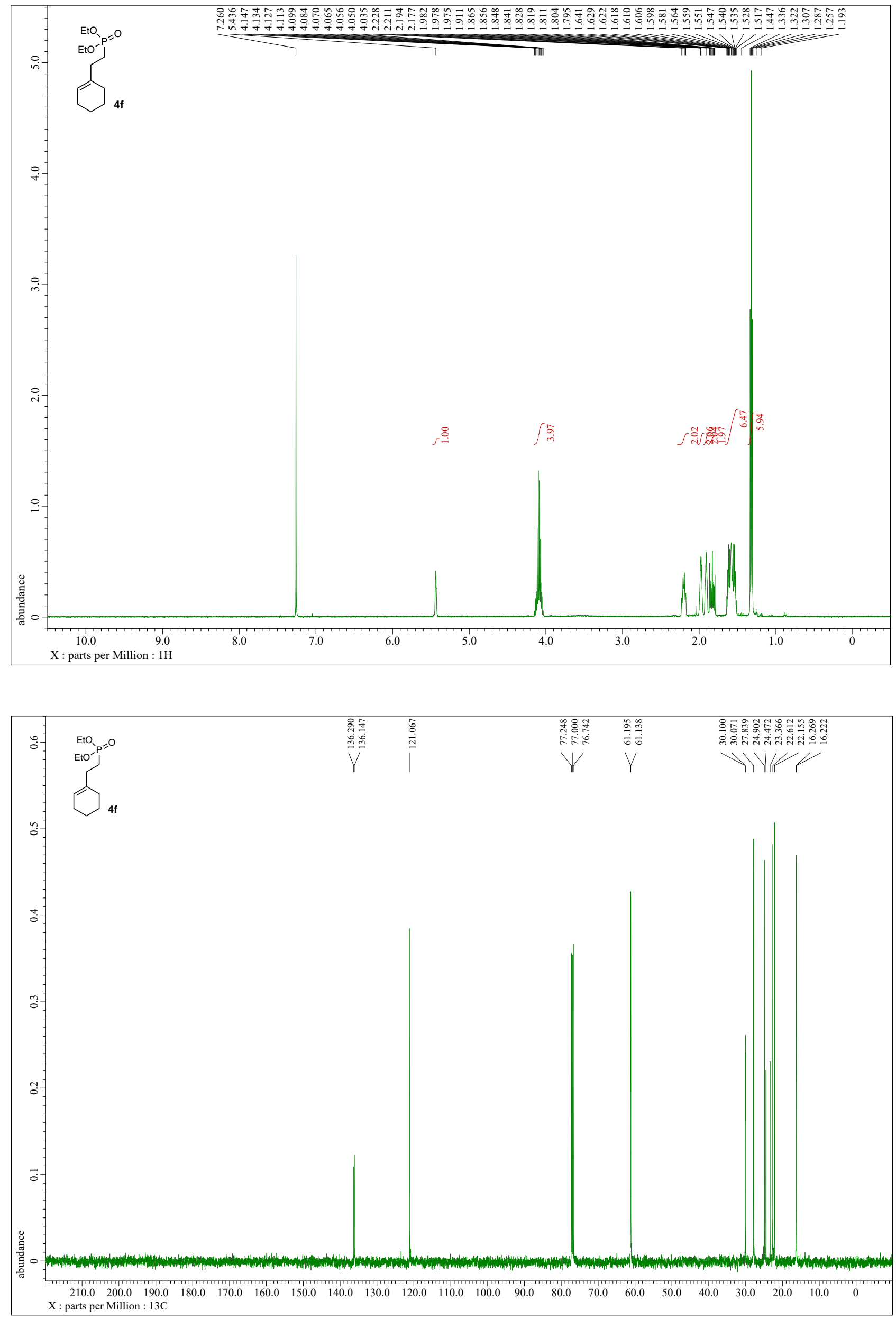


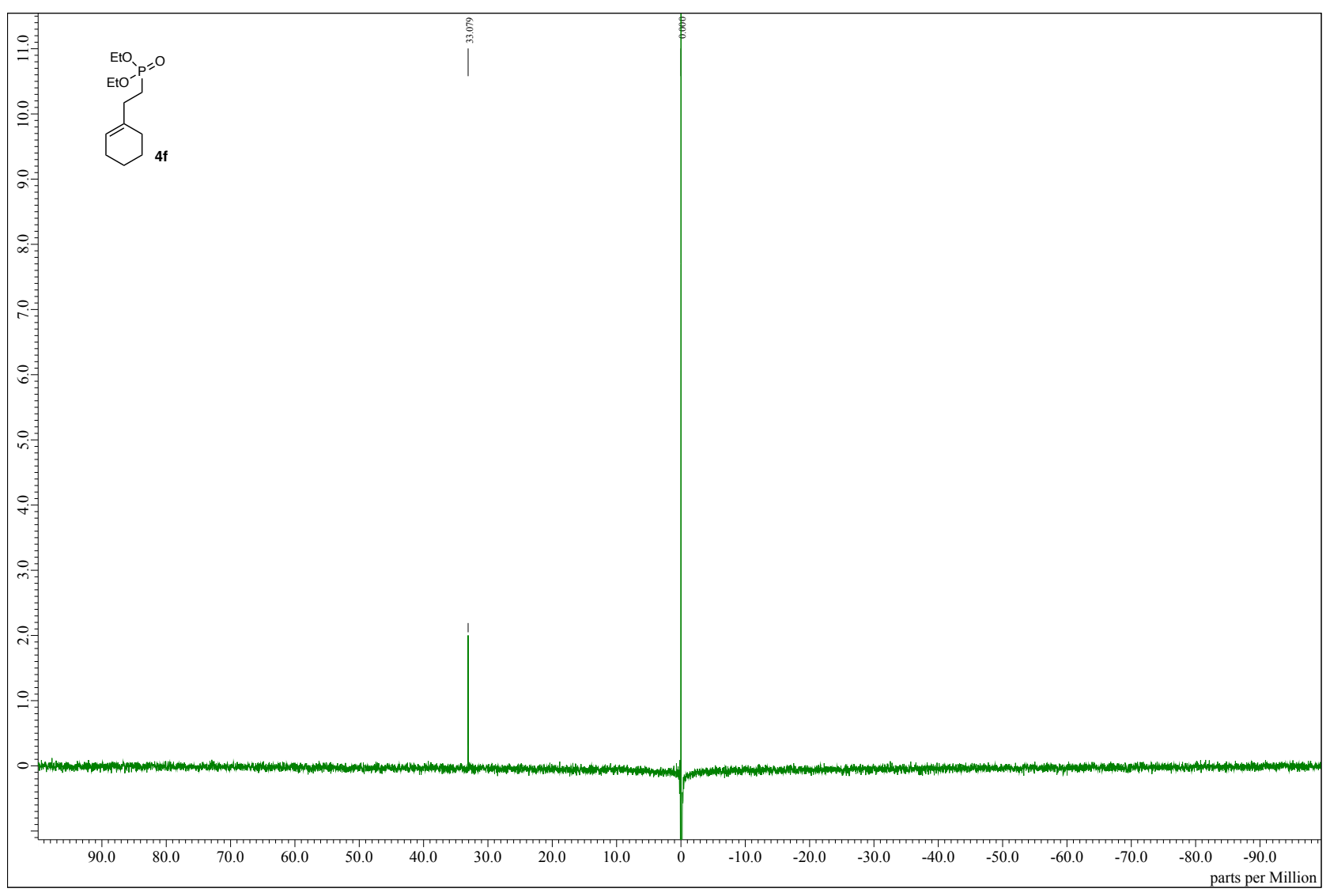


NMR spectra of $\mathbf{S 7}\left[{ }^{1} \mathrm{H}(500 \mathrm{MHz}),{ }^{13} \mathrm{C}(126 \mathrm{MHz})\right.$, and ${ }^{31} \mathrm{P}(202 \mathrm{MHz})$ in $\left.\mathrm{CDCl}_{3}\right]$
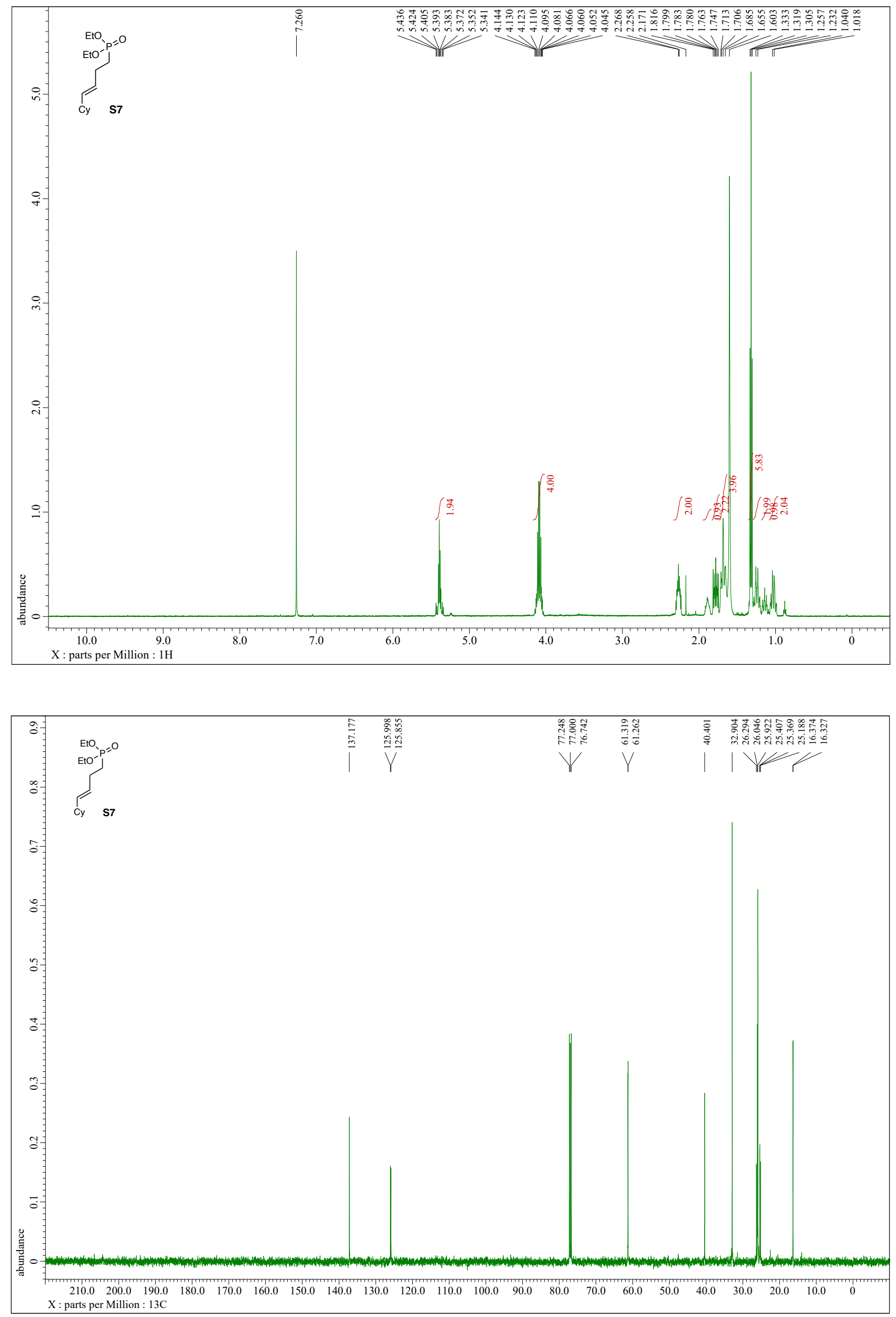


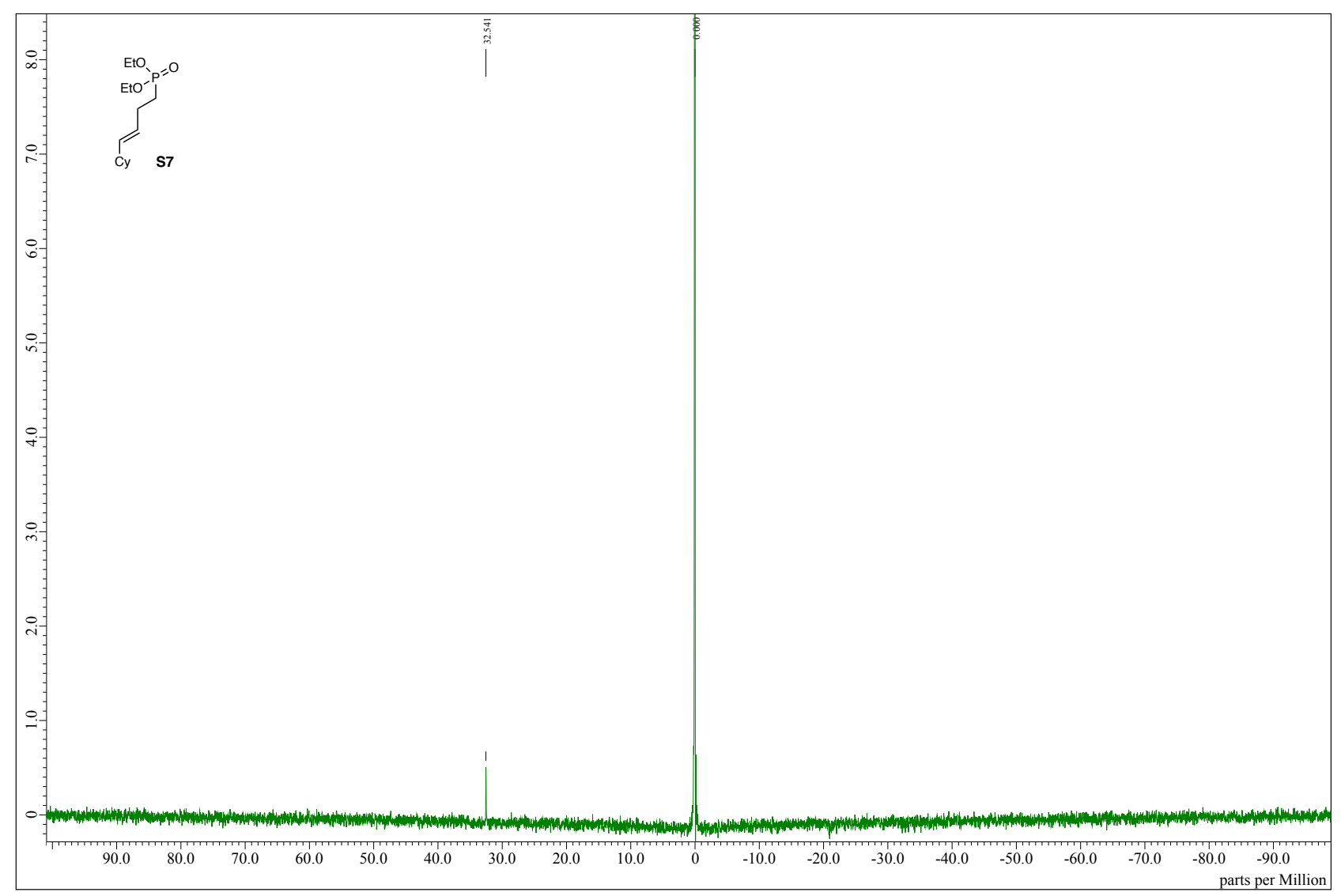


NMR spectra of $\mathbf{S 8}\left[{ }^{1} \mathrm{H}(500 \mathrm{MHz}),{ }^{13} \mathrm{C}(126 \mathrm{MHz})\right.$, and ${ }^{31} \mathrm{P}(202 \mathrm{MHz})$ in $\left.\mathrm{CDCl}_{3}\right]$
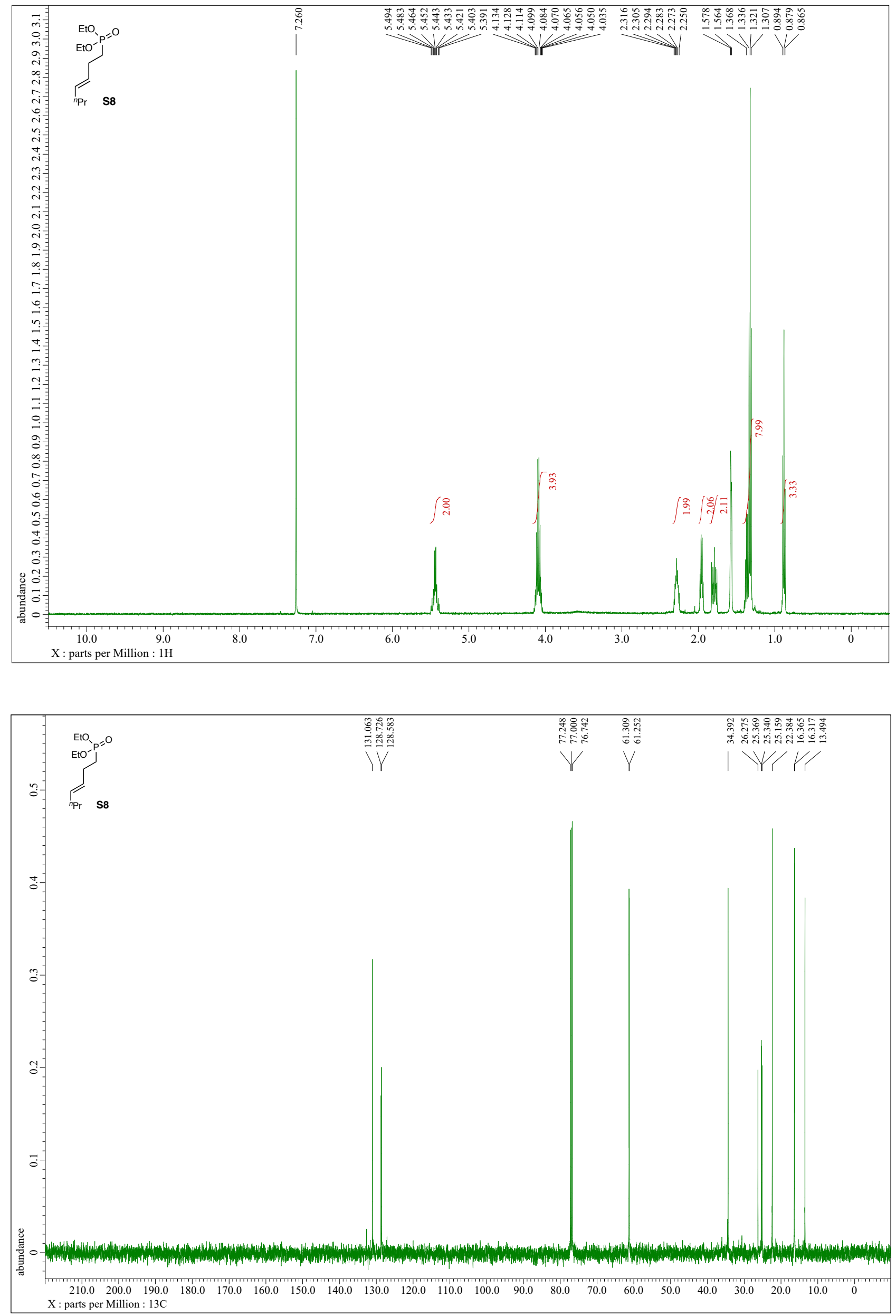


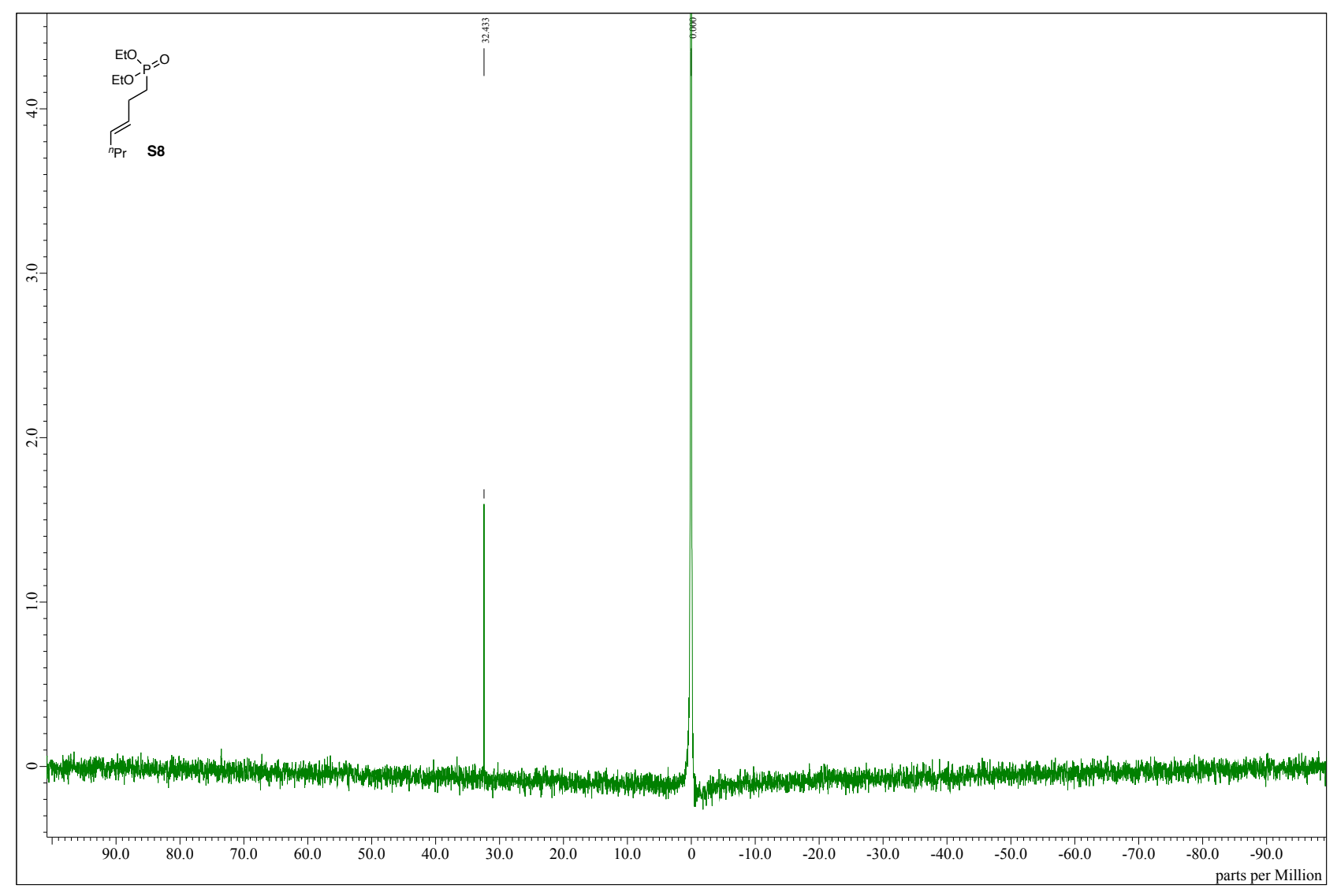


NMR spectra of $(1 E, 3 E)-5\left[{ }^{1} \mathrm{H}(500 \mathrm{MHz}),{ }^{13} \mathrm{C}(126 \mathrm{MHz})\right.$, and ${ }^{31} \mathrm{P}(202 \mathrm{MHz})$ in $\left.\mathrm{CDCl}_{3}\right]$
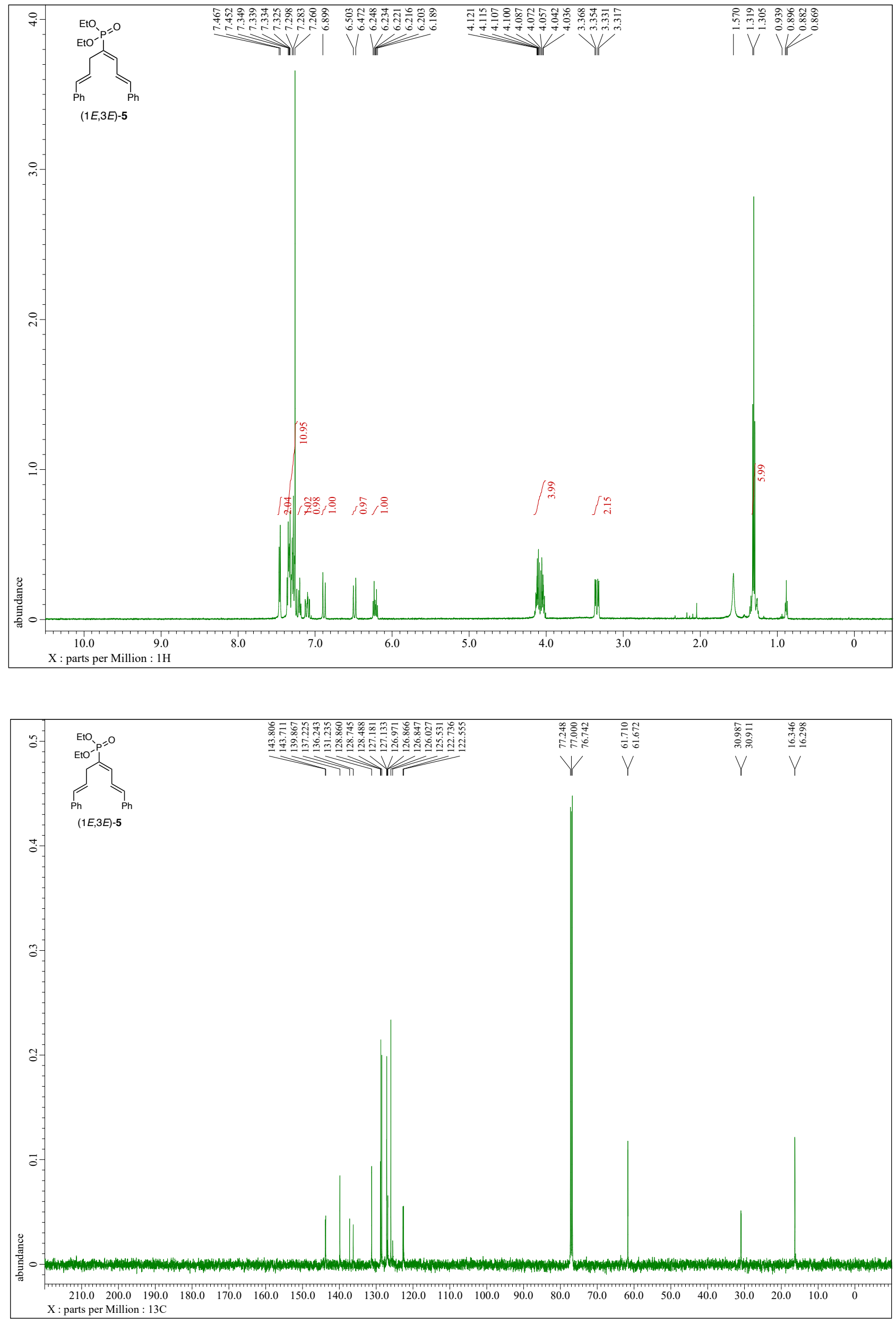


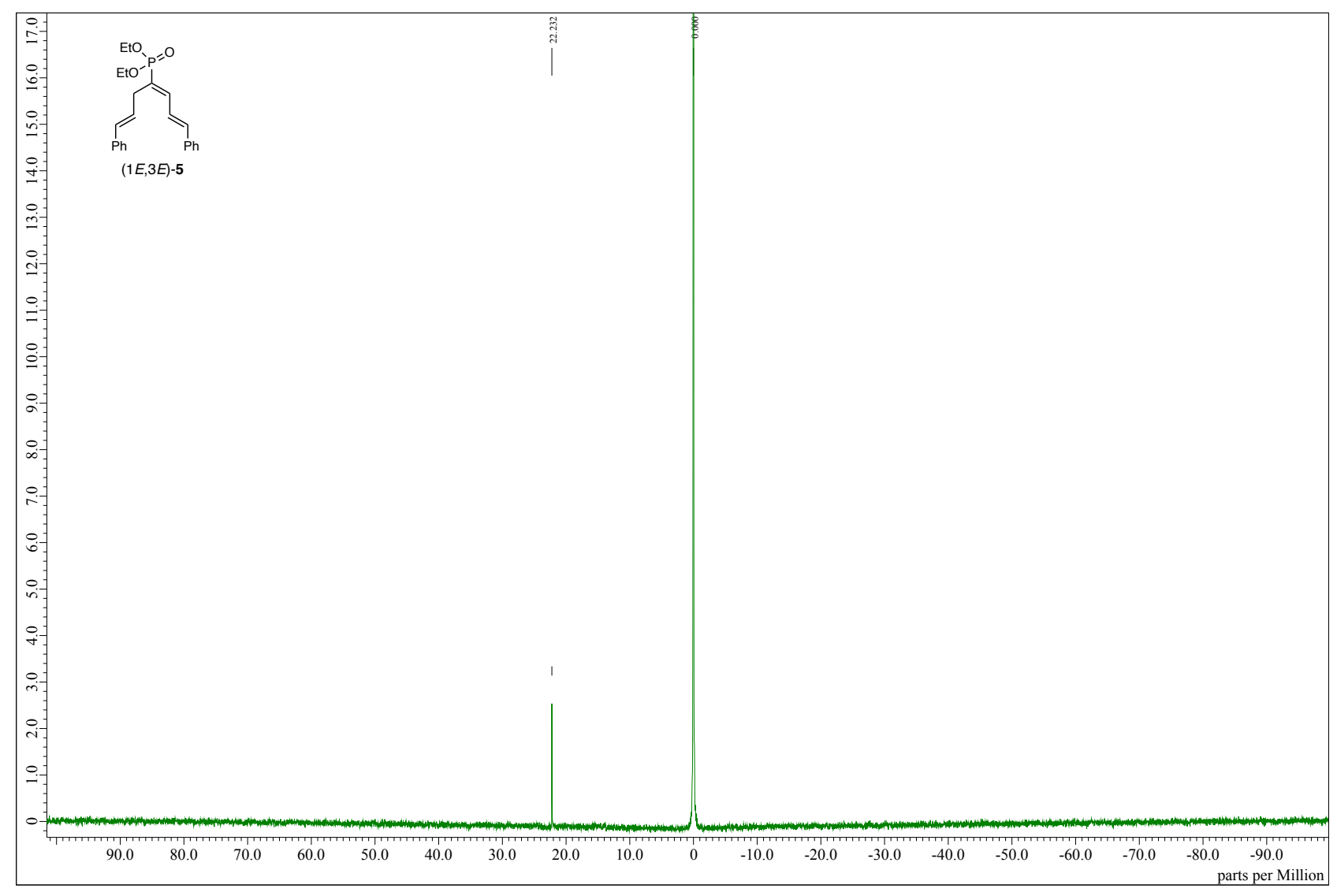


NMR spectra of (1E,3Z)-5 [ ${ }^{1} \mathrm{H}(500 \mathrm{MHz}),{ }^{13} \mathrm{C}(126 \mathrm{MHz})$, and ${ }^{31} \mathrm{P}(202 \mathrm{MHz})$ in $\left.\mathrm{CDCl}_{3}\right]$
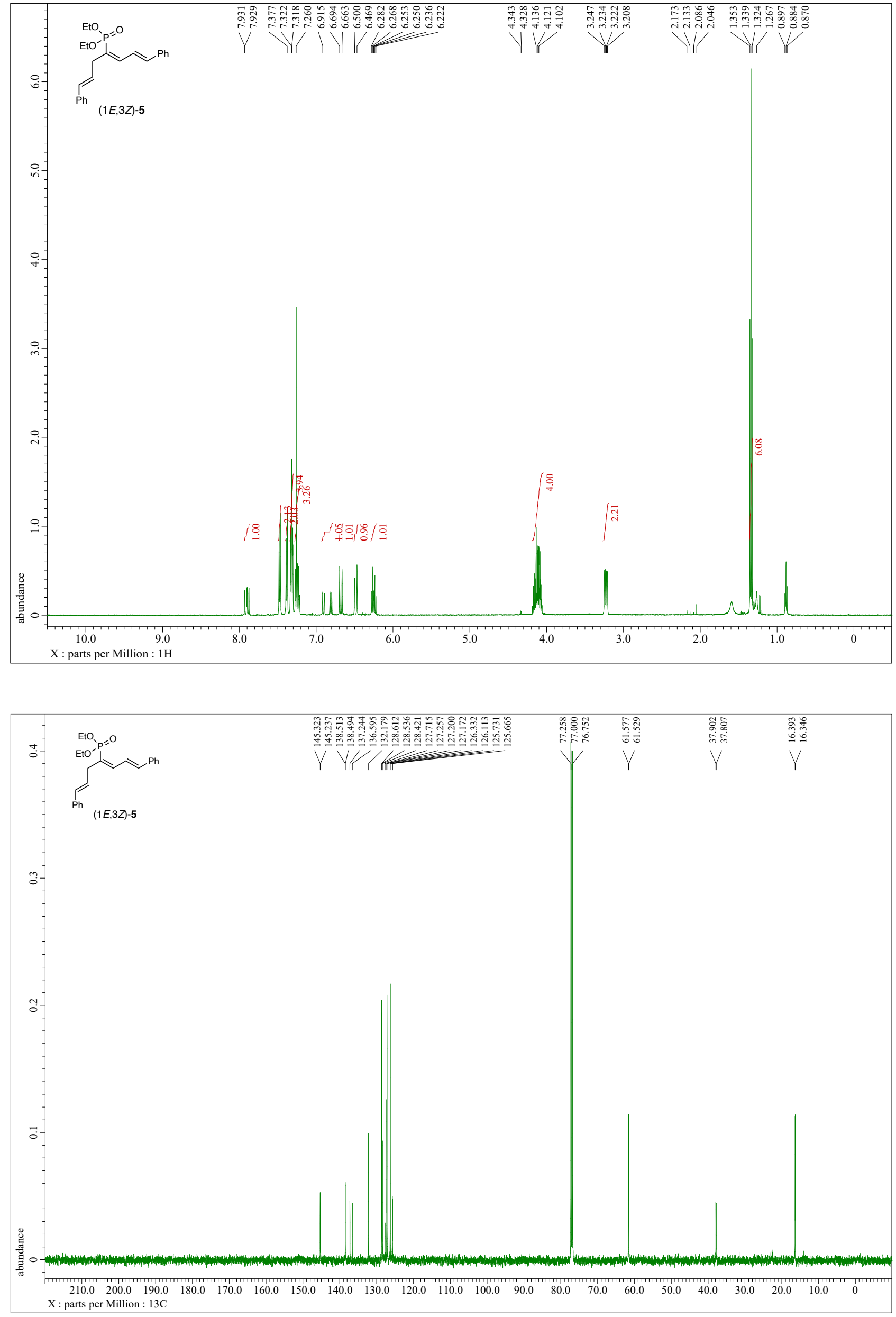


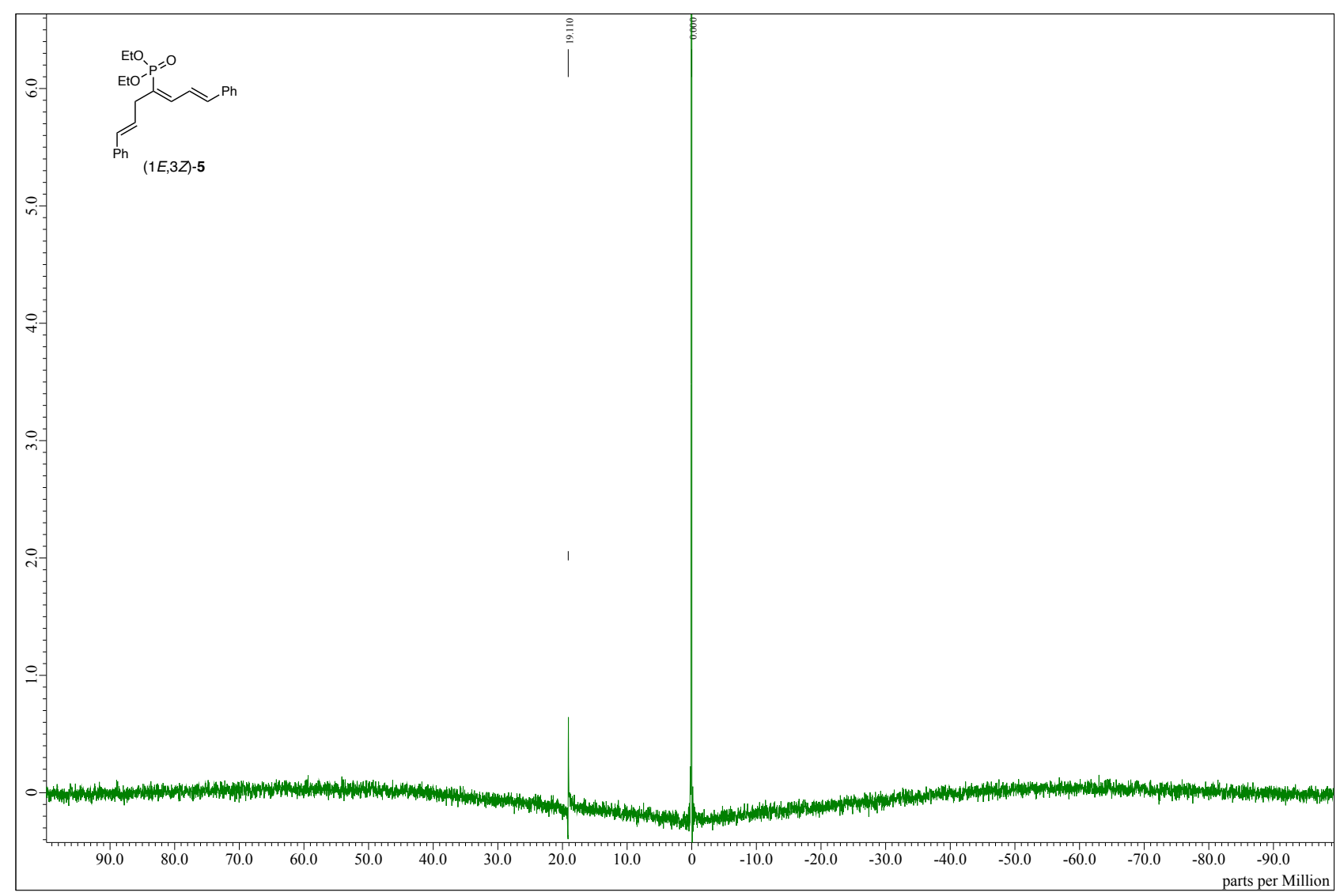


NMR spectra of $(1 Z, 3 E)-5\left[{ }^{1} \mathrm{H}(500 \mathrm{MHz}),{ }^{13} \mathrm{C}(126 \mathrm{MHz})\right.$, and ${ }^{31} \mathrm{P}(202 \mathrm{MHz})$ in $\left.\mathrm{CDCl}_{3}\right]$
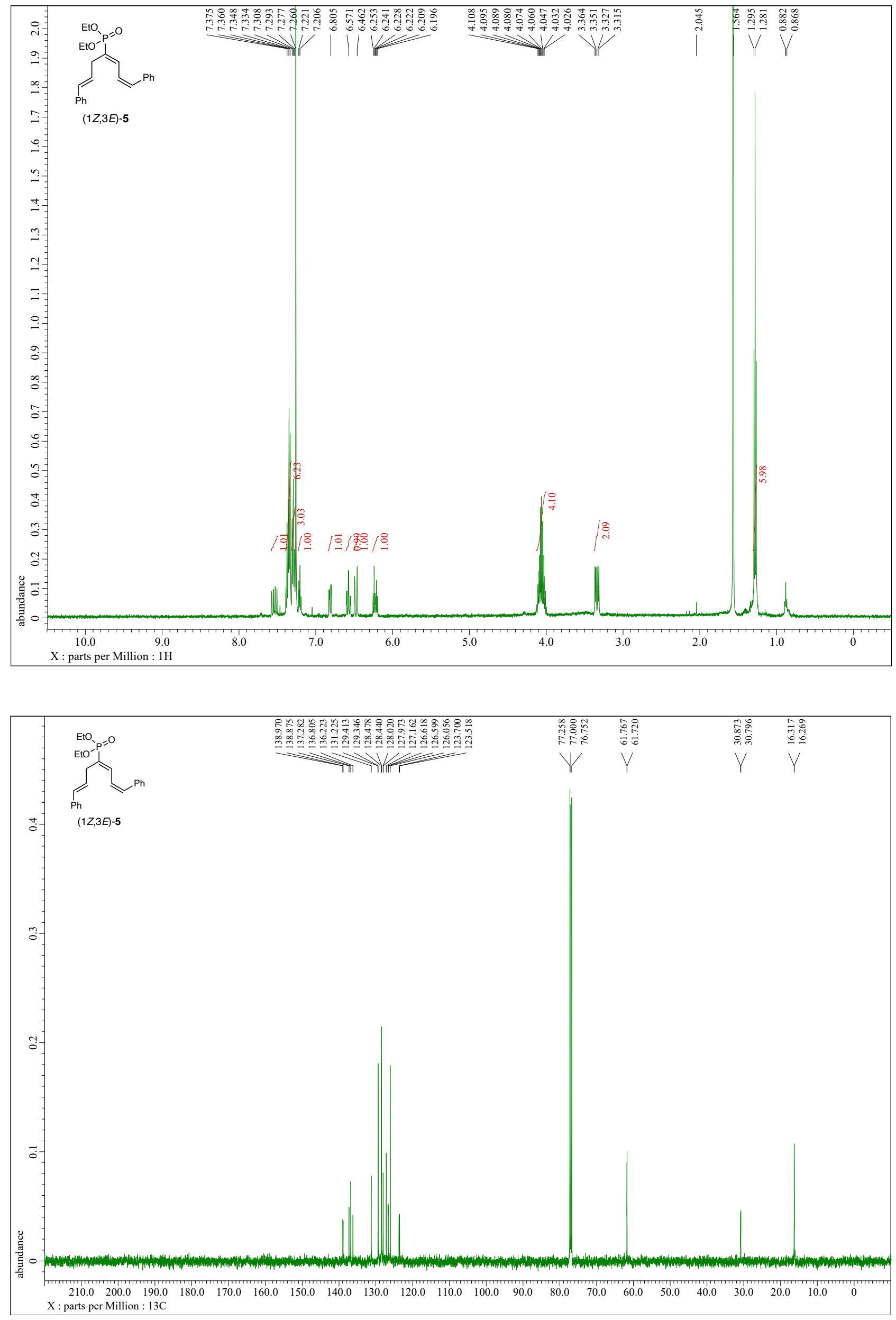


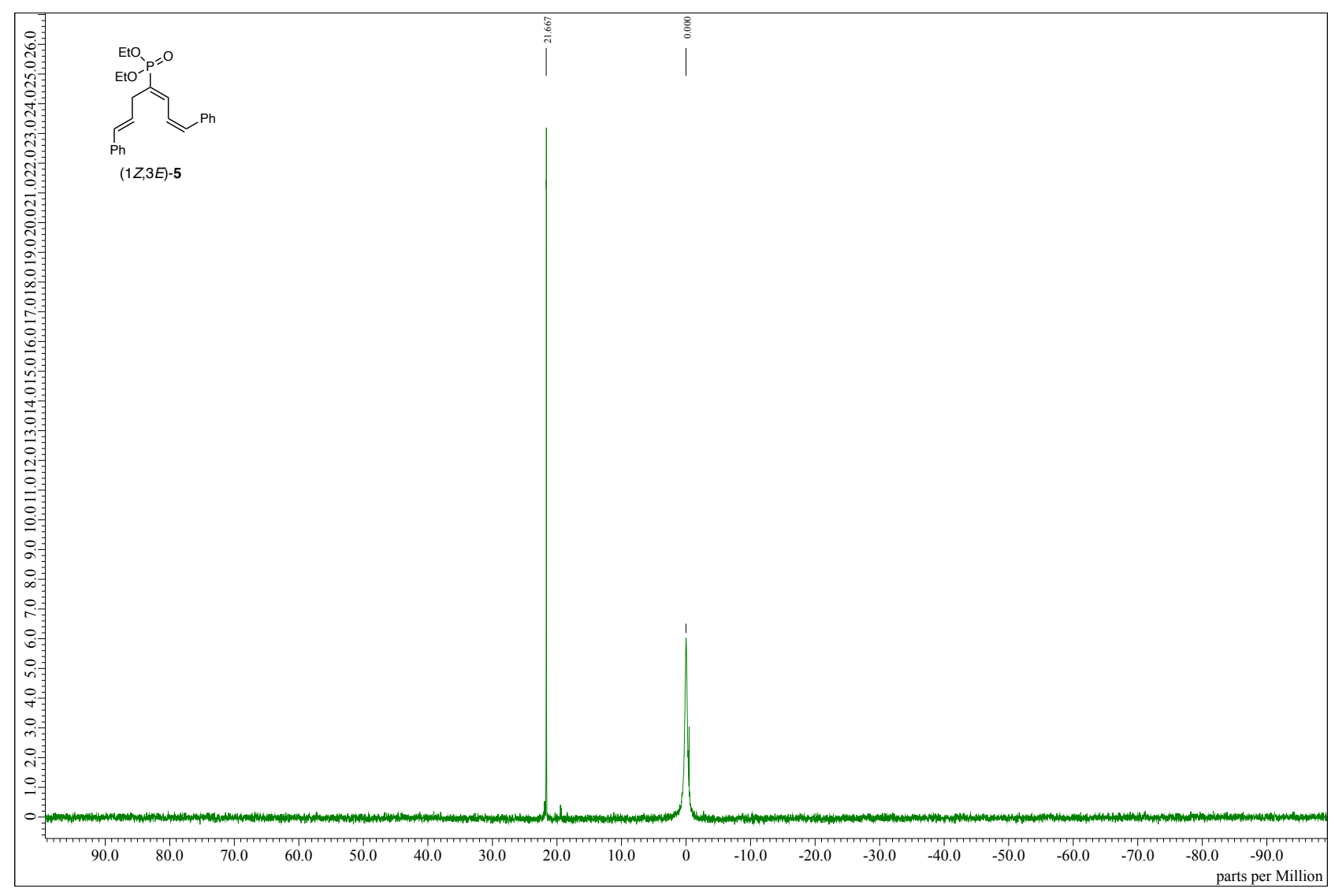


NMR spectra of (1Z,3Z)-5 [ ${ }^{1} \mathrm{H}(500 \mathrm{MHz}),{ }^{13} \mathrm{C}(126 \mathrm{MHz})$, and ${ }^{31} \mathrm{P}(202 \mathrm{MHz})$ in $\left.\mathrm{CDCl}_{3}\right]$
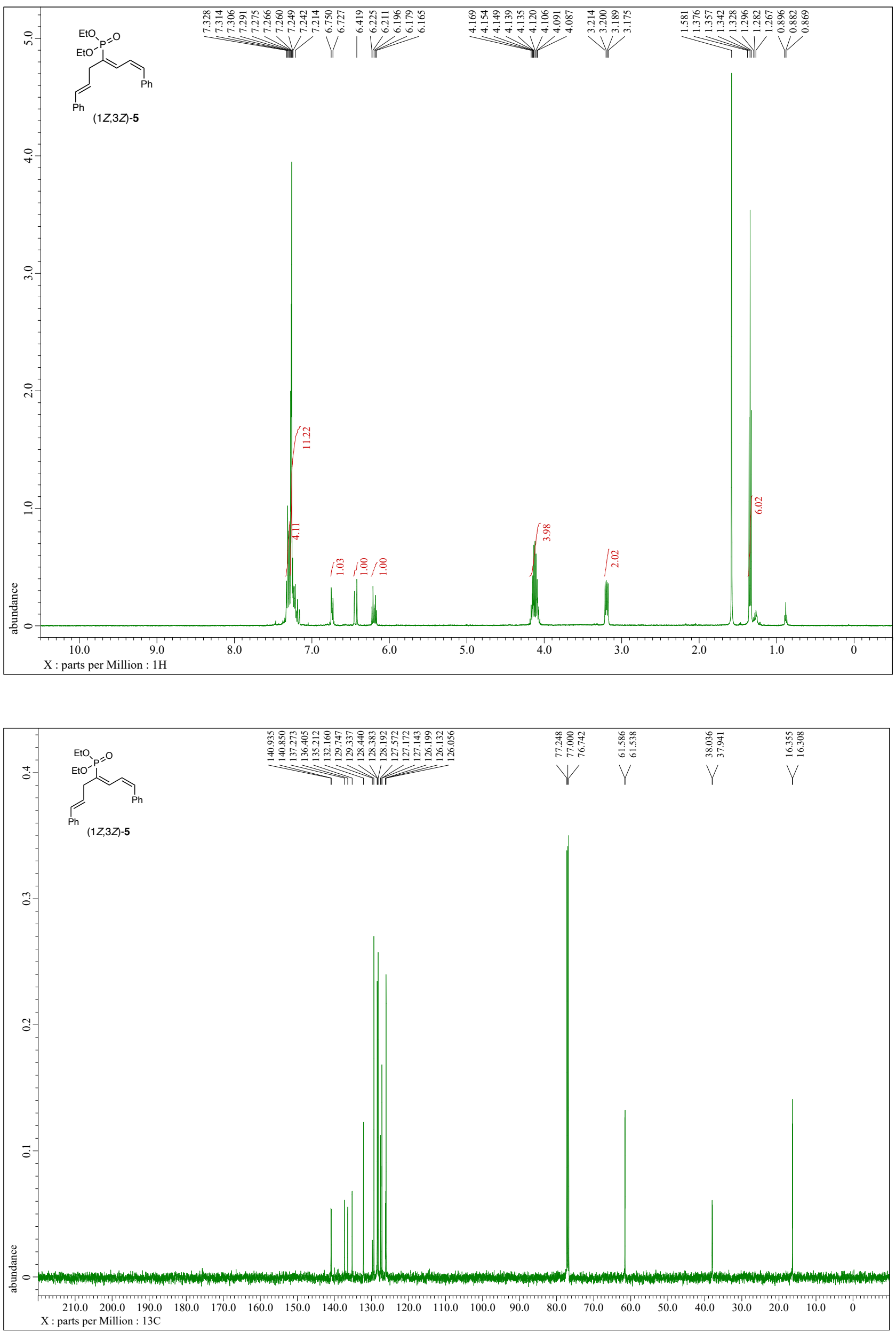


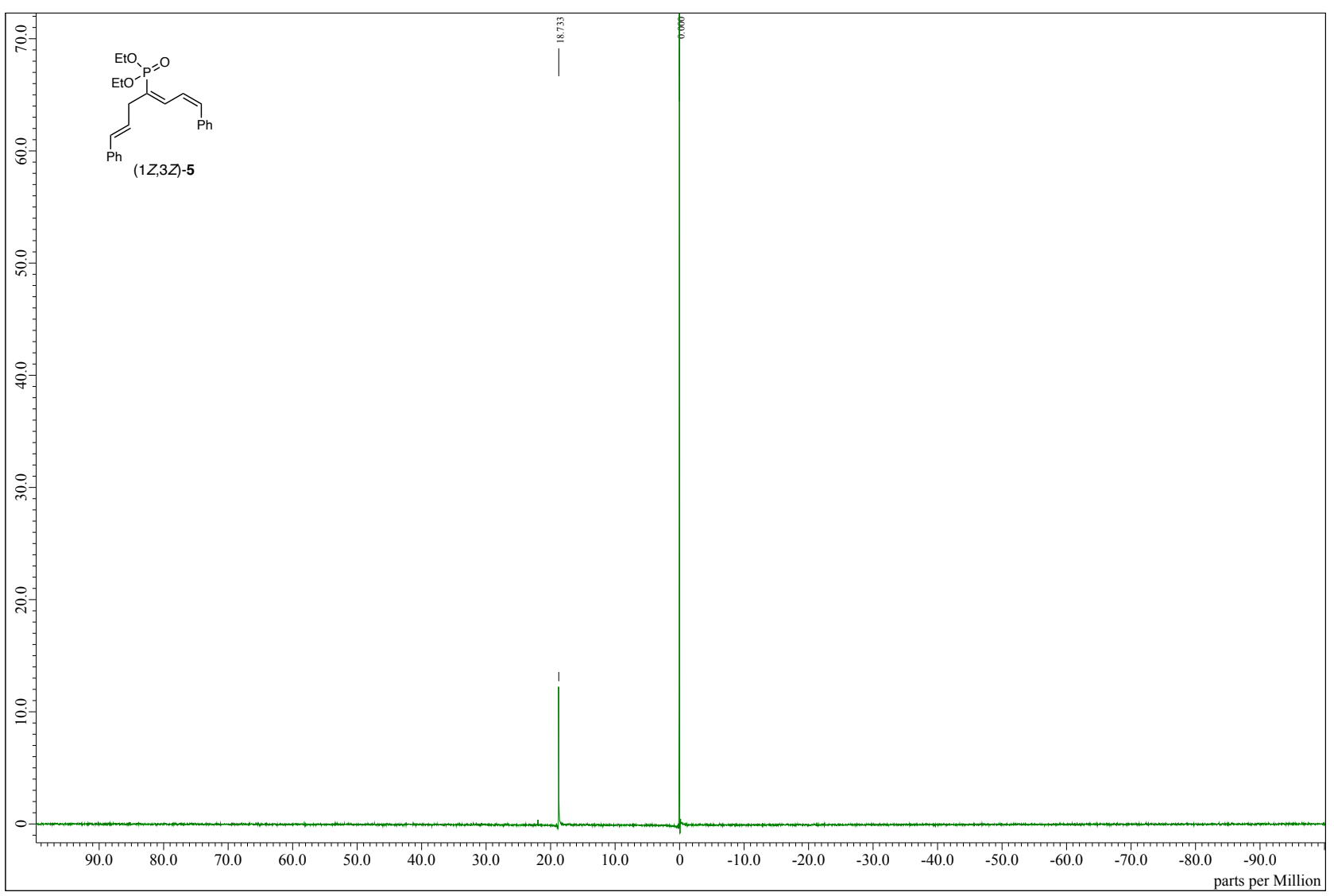


NMR spectra of (3Z)-10a $\left[{ }^{1} \mathrm{H}(500 \mathrm{MHz}),{ }^{13} \mathrm{C}(126 \mathrm{MHz})\right.$, and ${ }^{31} \mathrm{P}(202 \mathrm{MHz})$ in $\left.\mathrm{CDCl}_{3}\right]$
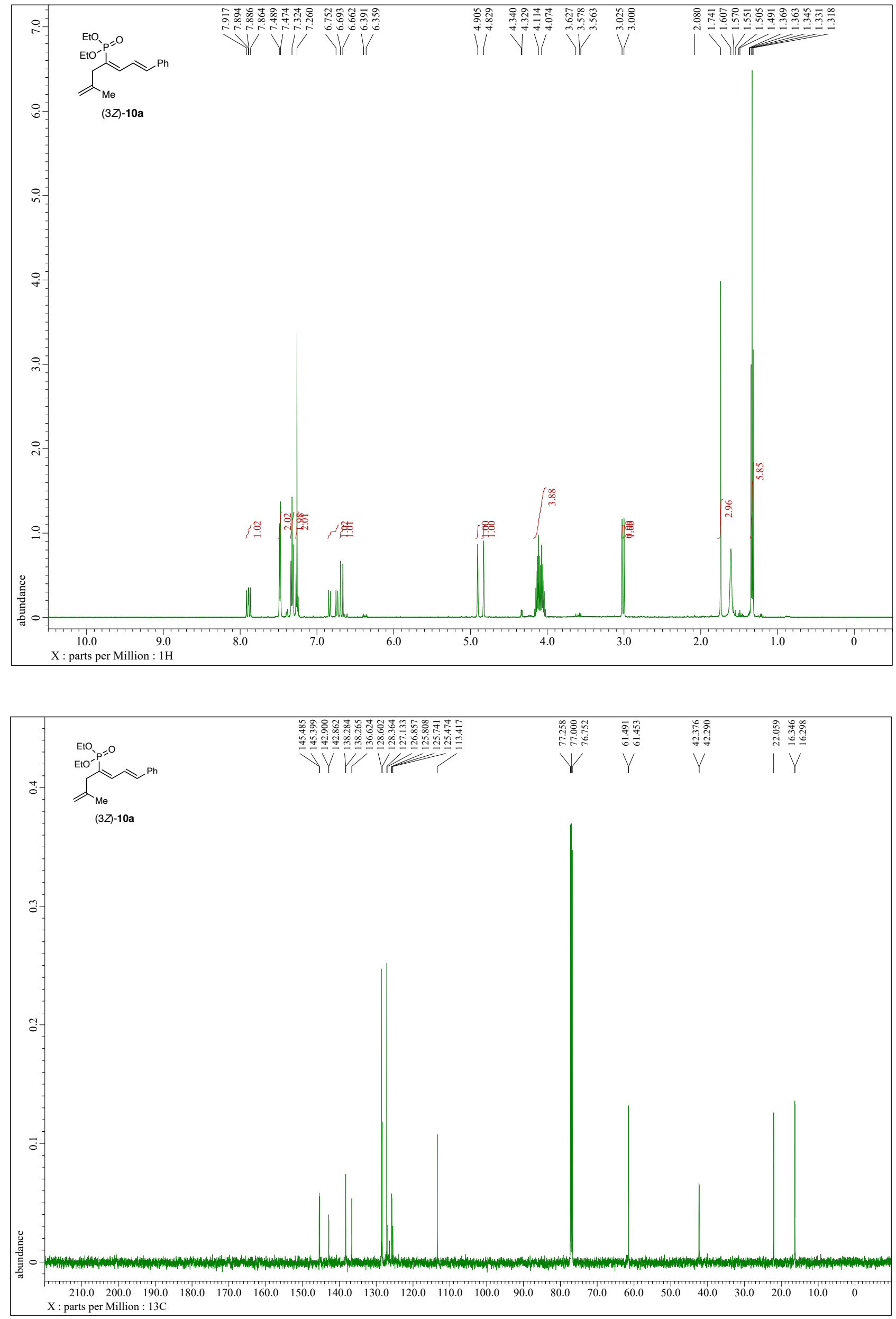


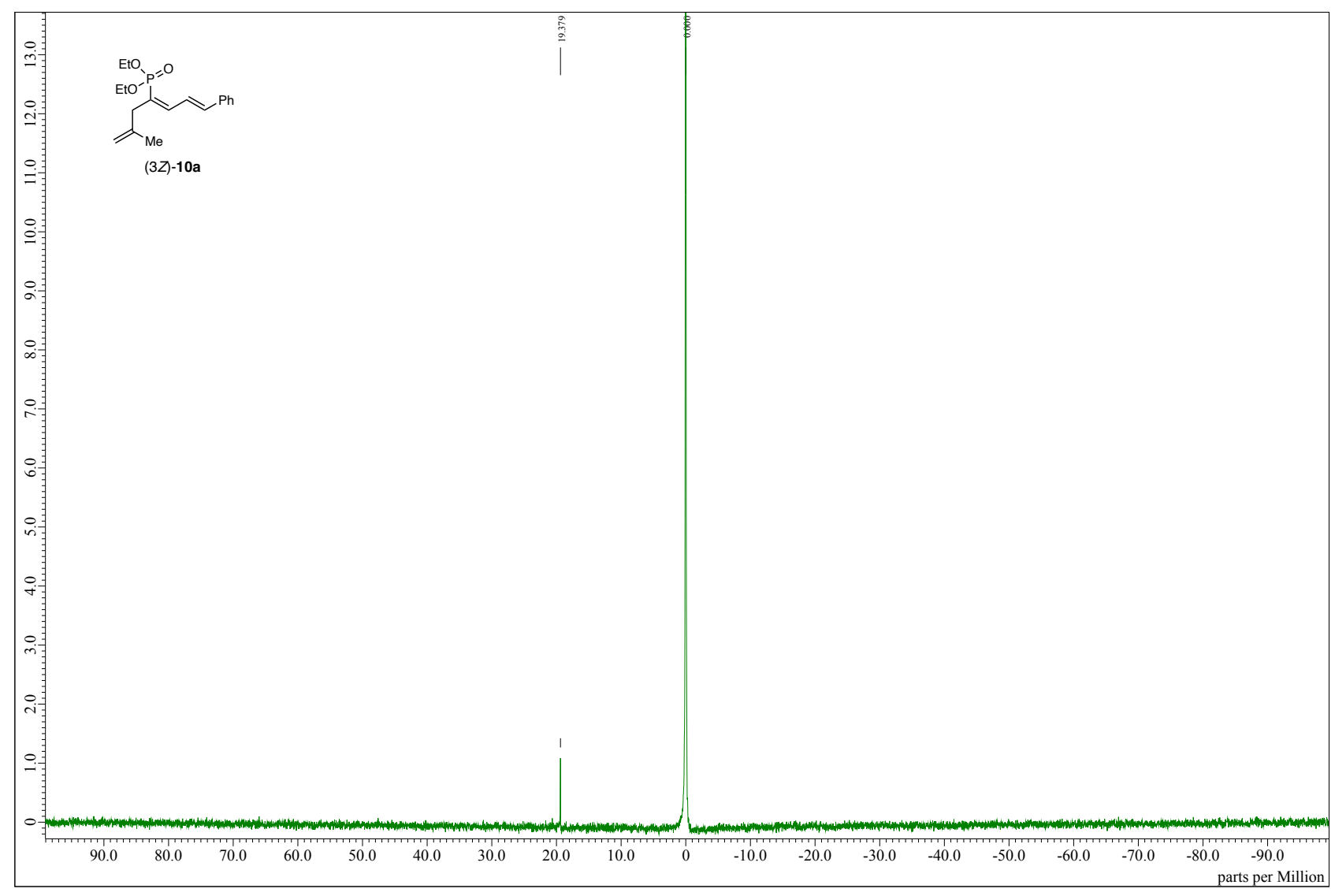


NMR spectra of $(3 E)-10 b\left[{ }^{1} \mathrm{H}(500 \mathrm{MHz}),{ }^{13} \mathrm{C}(126 \mathrm{MHz})\right.$, and ${ }^{31} \mathrm{P}(202 \mathrm{MHz})$ in $\left.\mathrm{CDCl}_{3}\right]$
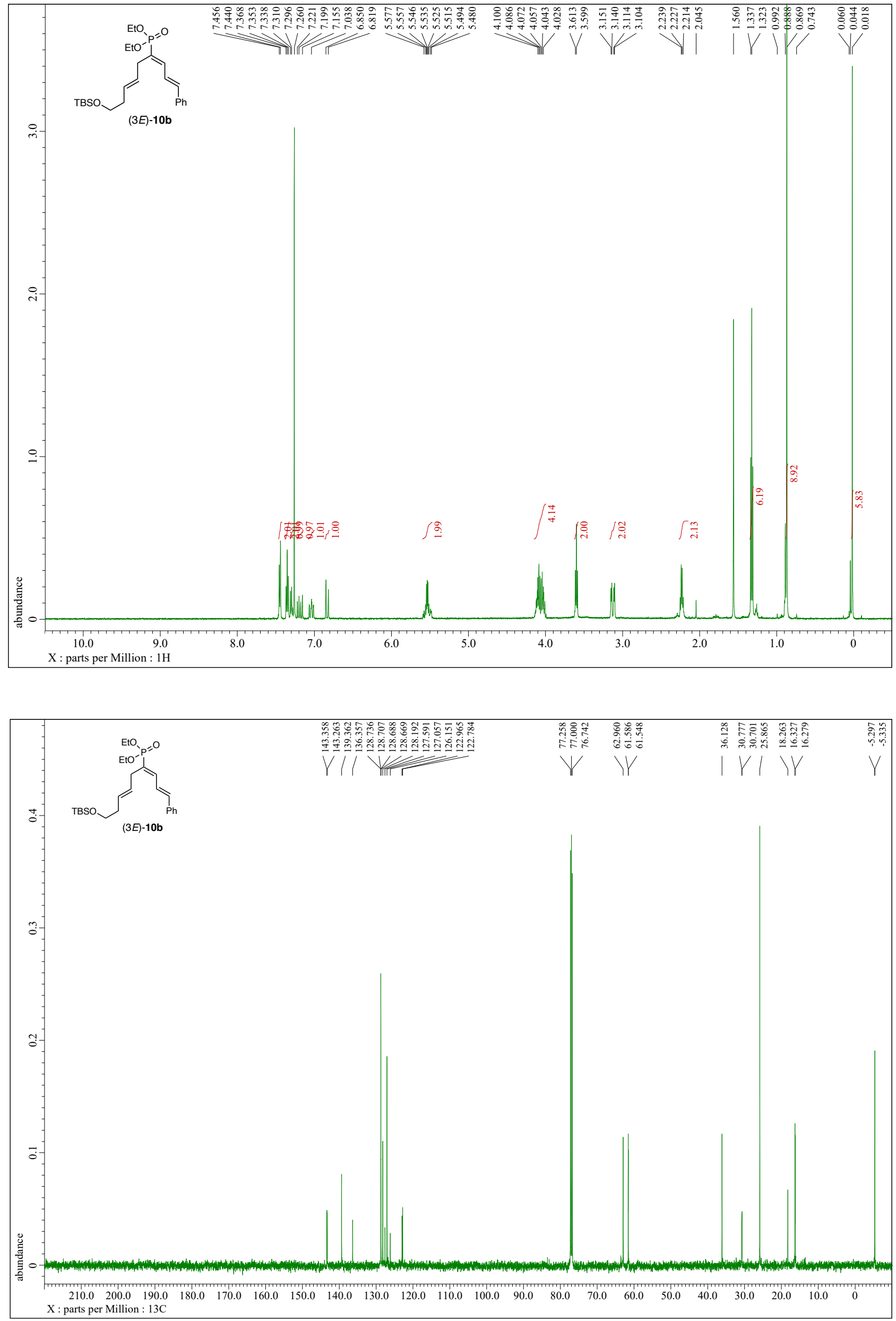


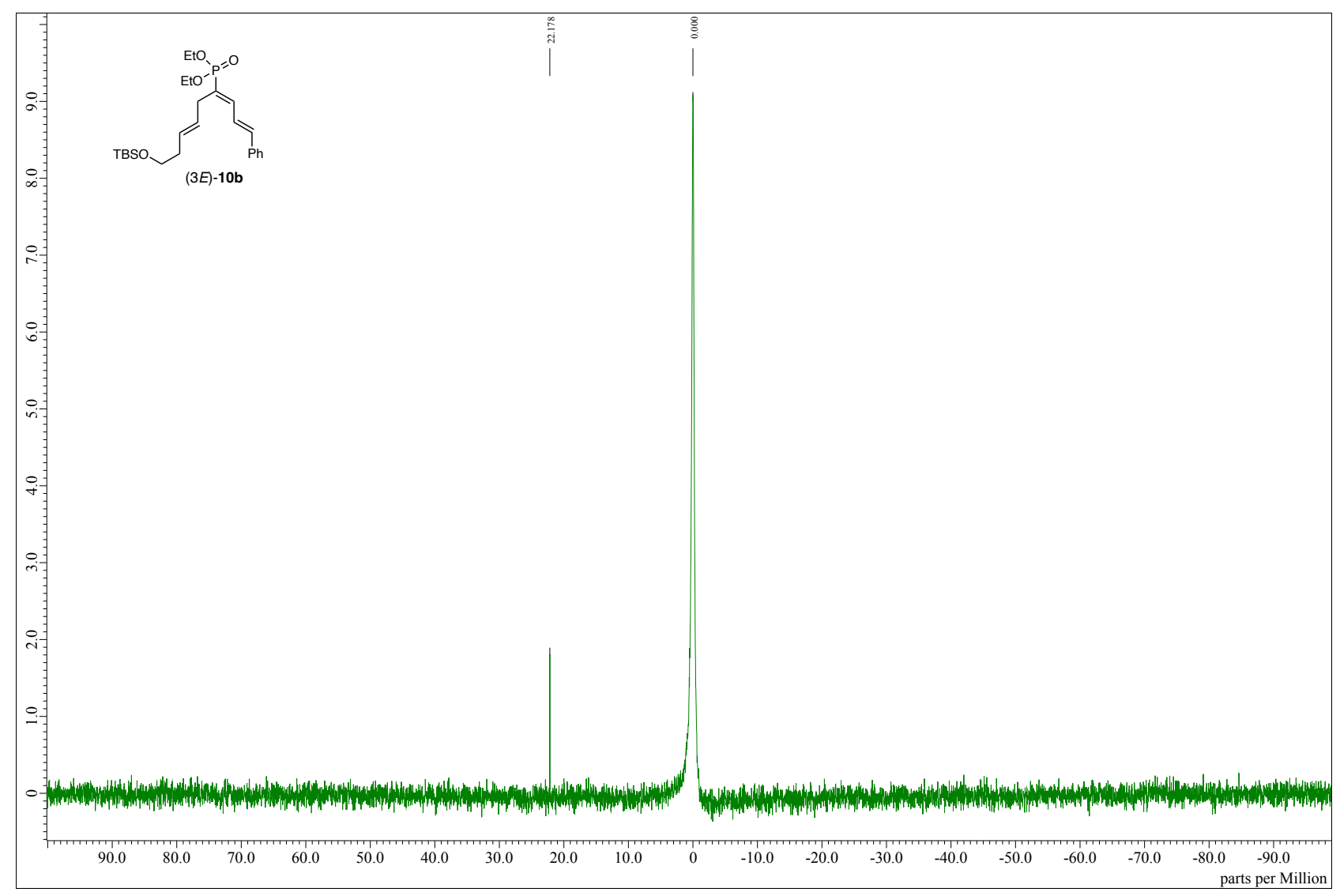


NMR spectra of (3Z)-10b $\left[{ }^{1} \mathrm{H}(500 \mathrm{MHz}),{ }^{13} \mathrm{C}(126 \mathrm{MHz})\right.$, and ${ }^{31} \mathrm{P}(202 \mathrm{MHz})$ in $\left.\mathrm{CDCl}_{3}\right]$
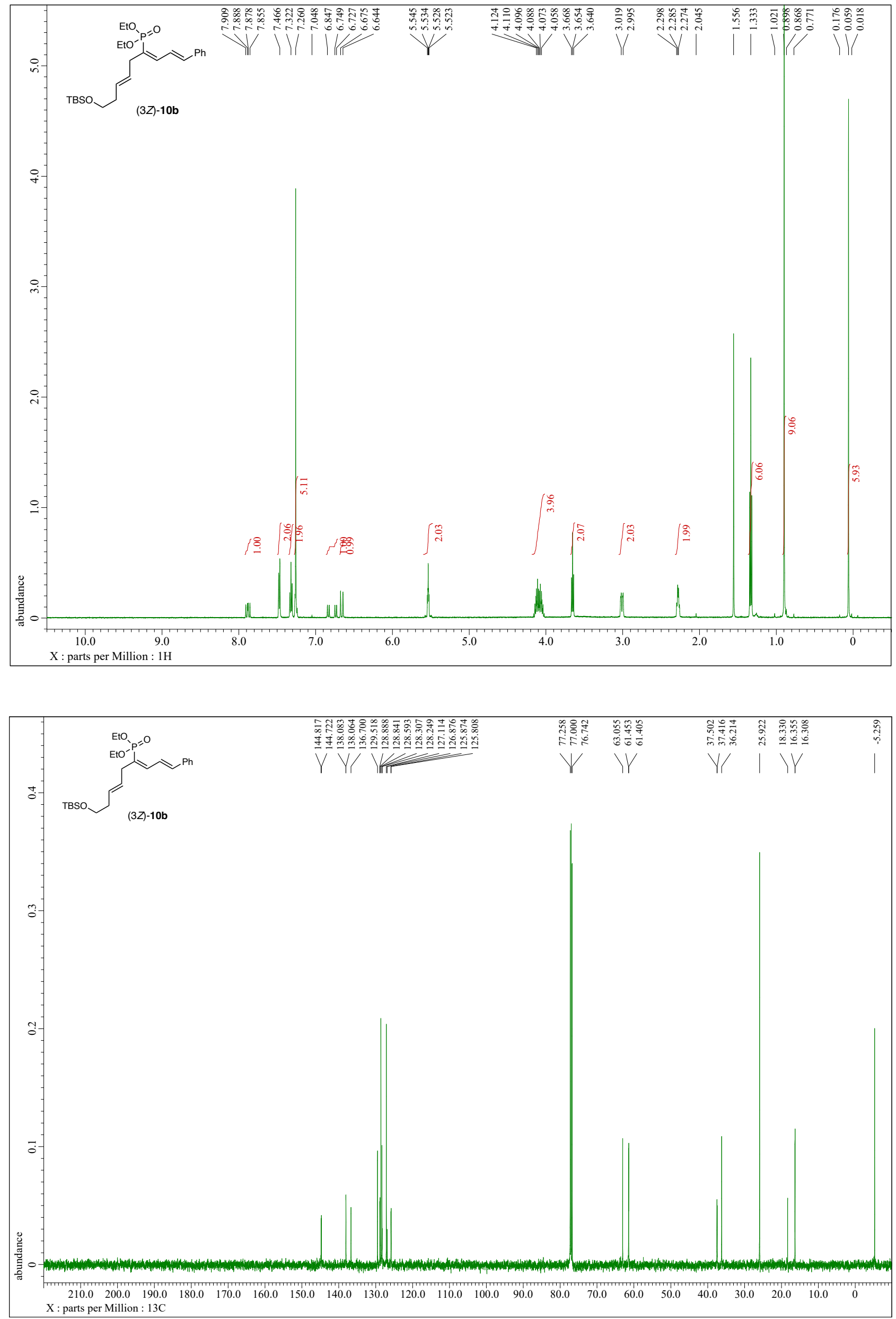


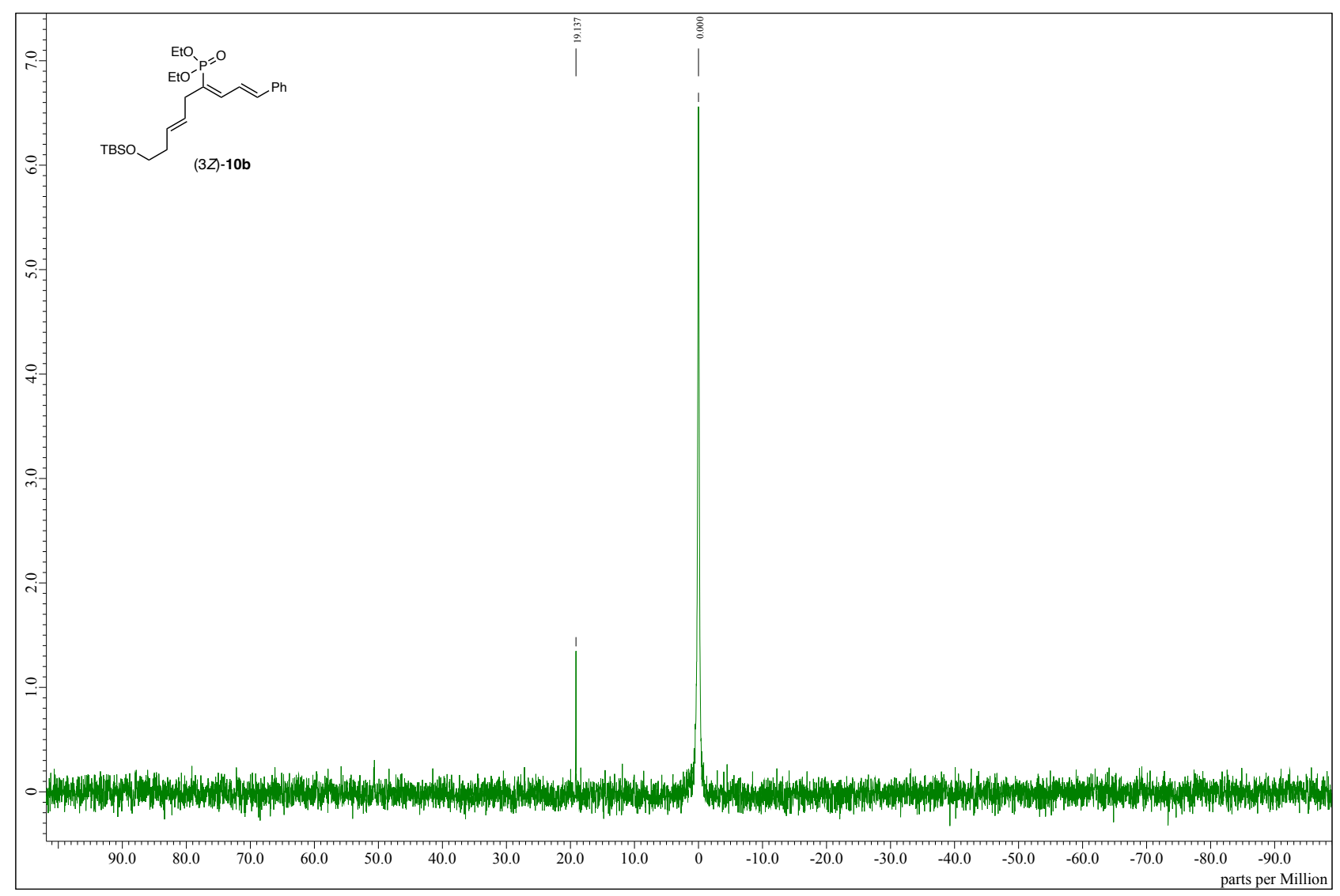


NMR spectra of (3E)-10c $\left[{ }^{1} \mathrm{H}(500 \mathrm{MHz}),{ }^{13} \mathrm{C}(126 \mathrm{MHz})\right.$, and ${ }^{31} \mathrm{P}(202 \mathrm{MHz})$ in $\left.\mathrm{CDCl}_{3}\right]$
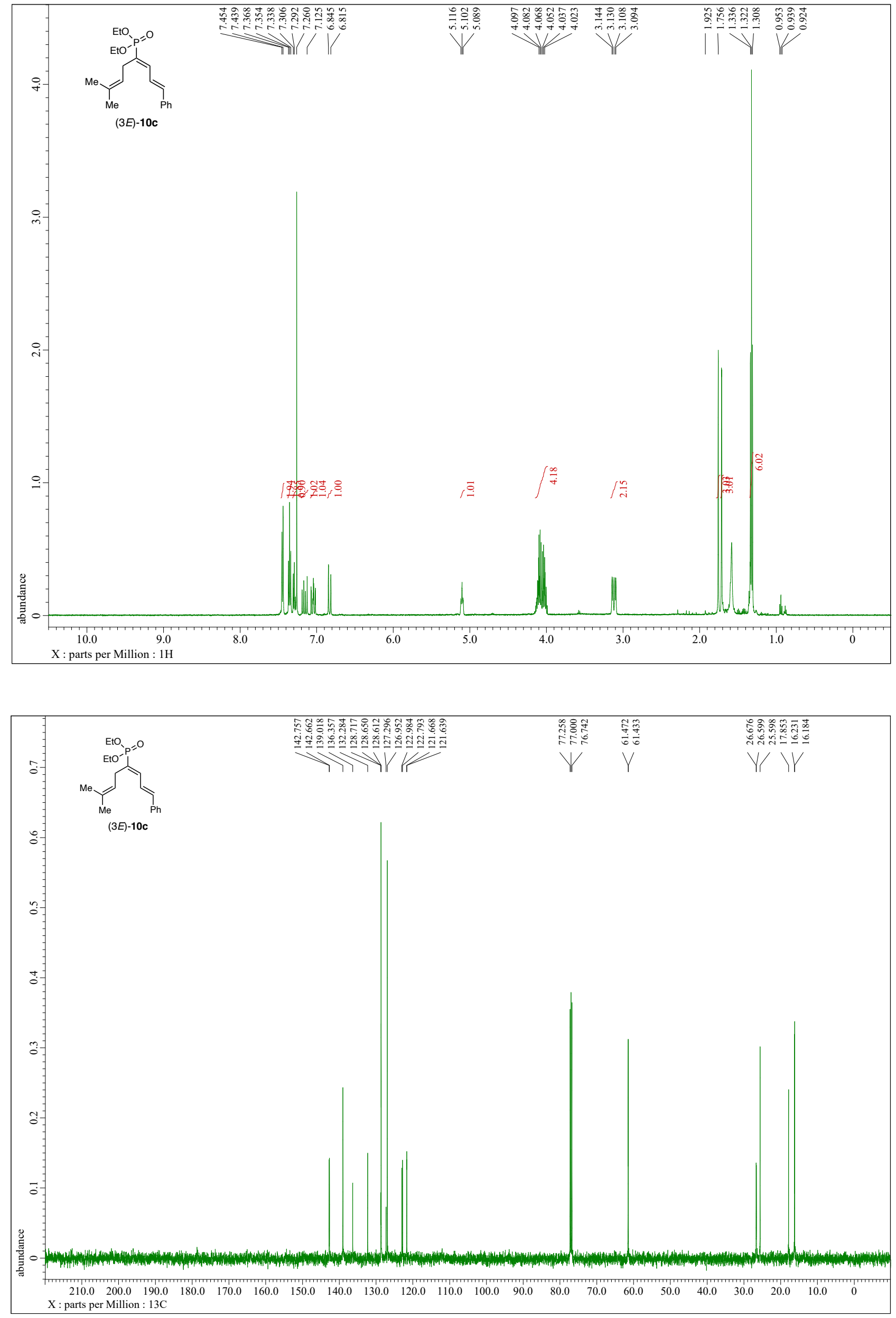


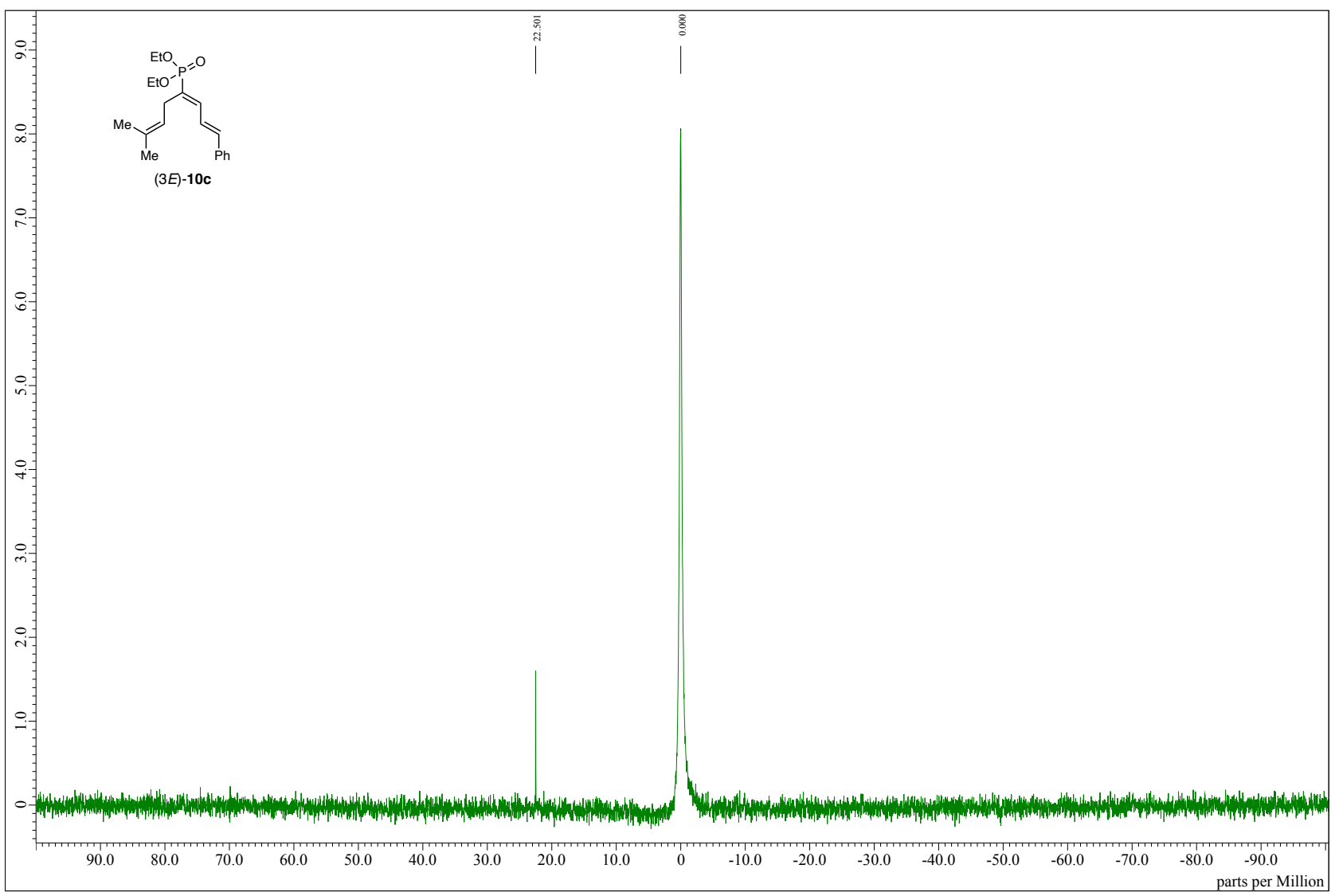


NMR spectra of (3Z)-10c $\left[{ }^{1} \mathrm{H}(500 \mathrm{MHz}),{ }^{13} \mathrm{C}(126 \mathrm{MHz})\right.$, and ${ }^{31} \mathrm{P}(202 \mathrm{MHz})$ in $\left.\mathrm{CDCl}_{3}\right]$
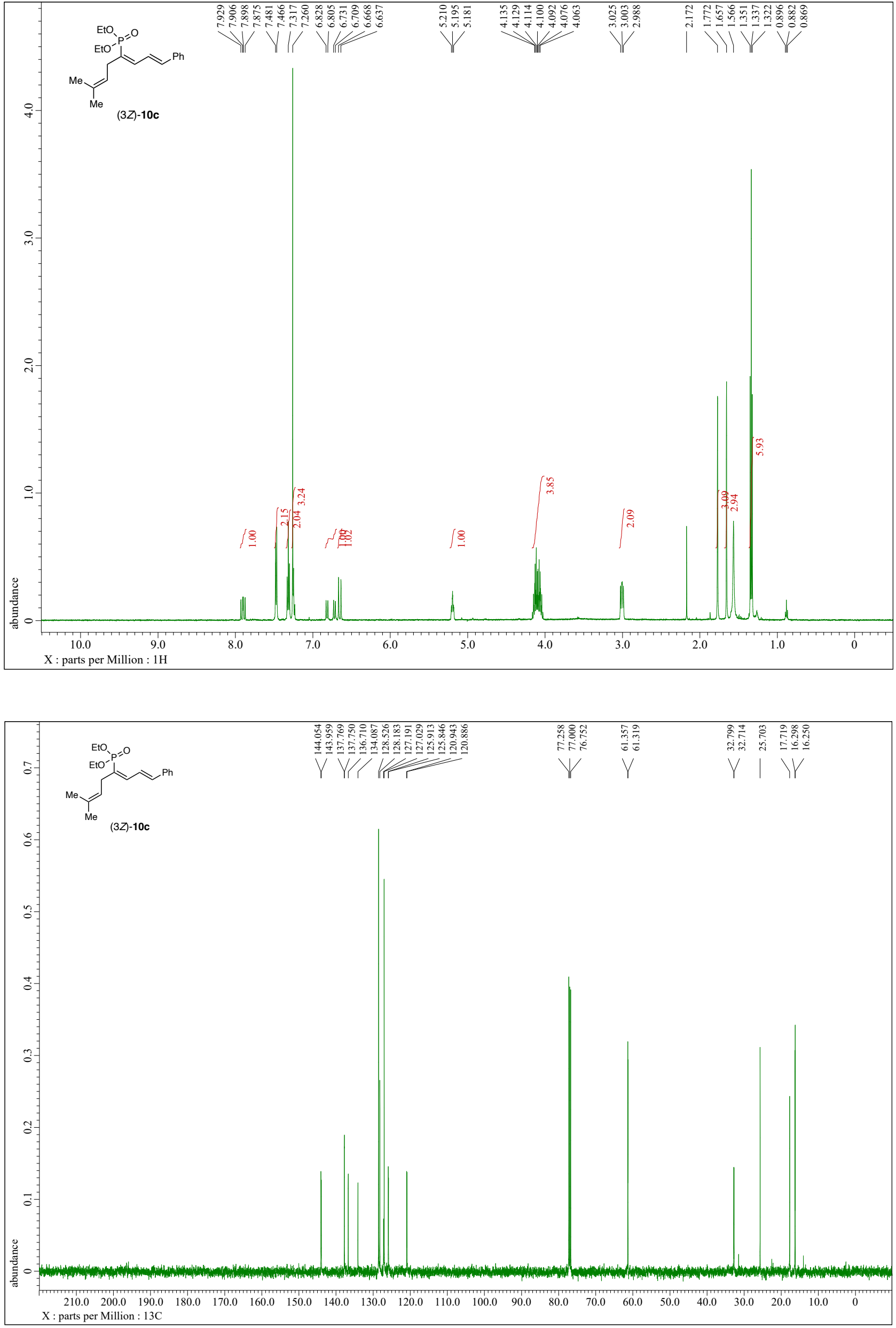


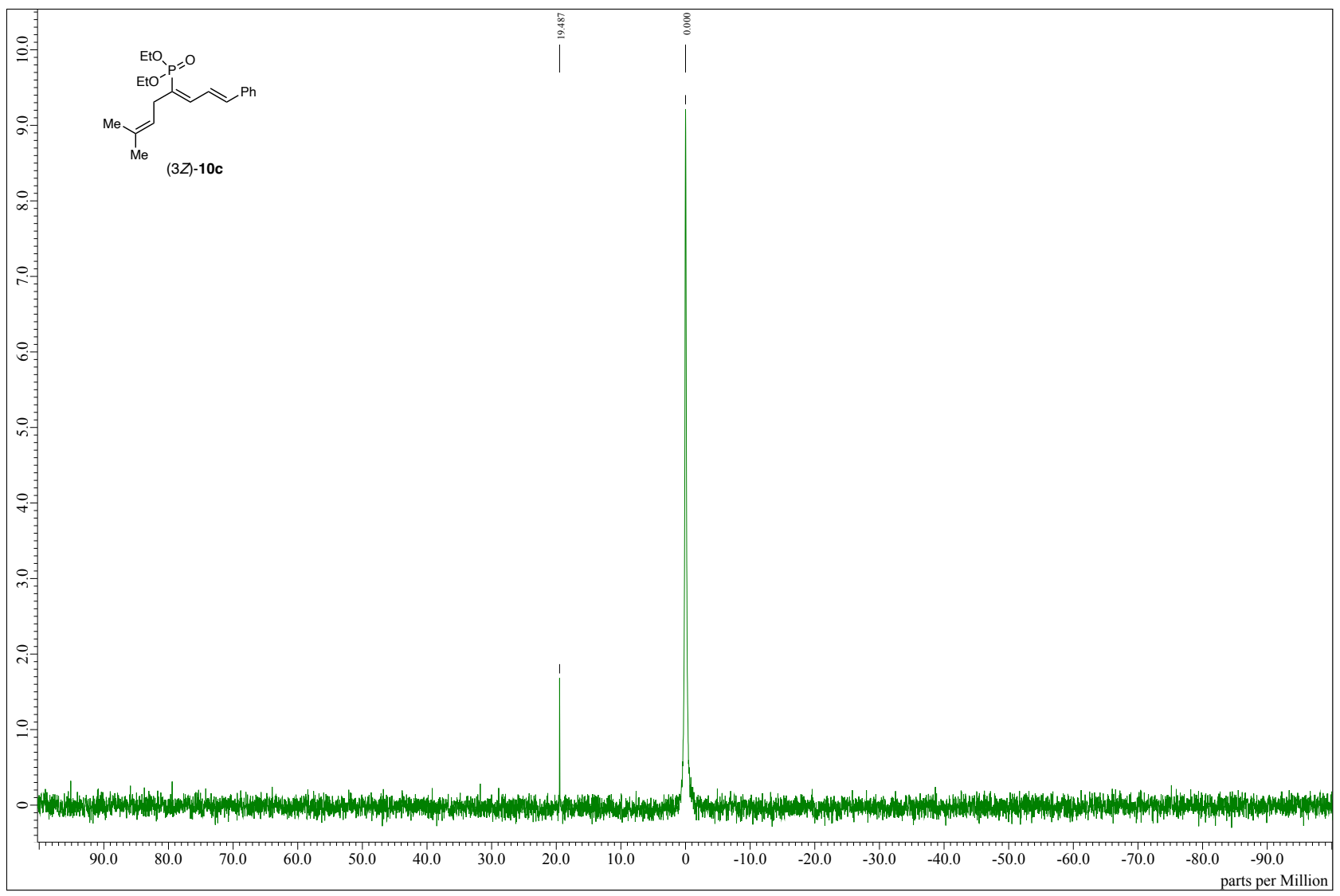


NMR spectra of (3Z)-10d $\left[{ }^{1} \mathrm{H}(500 \mathrm{MHz}),{ }^{13} \mathrm{C}(126 \mathrm{MHz})\right.$, and ${ }^{31} \mathrm{P}(202 \mathrm{MHz})$ in $\left.\mathrm{CDCl}_{3}\right]$
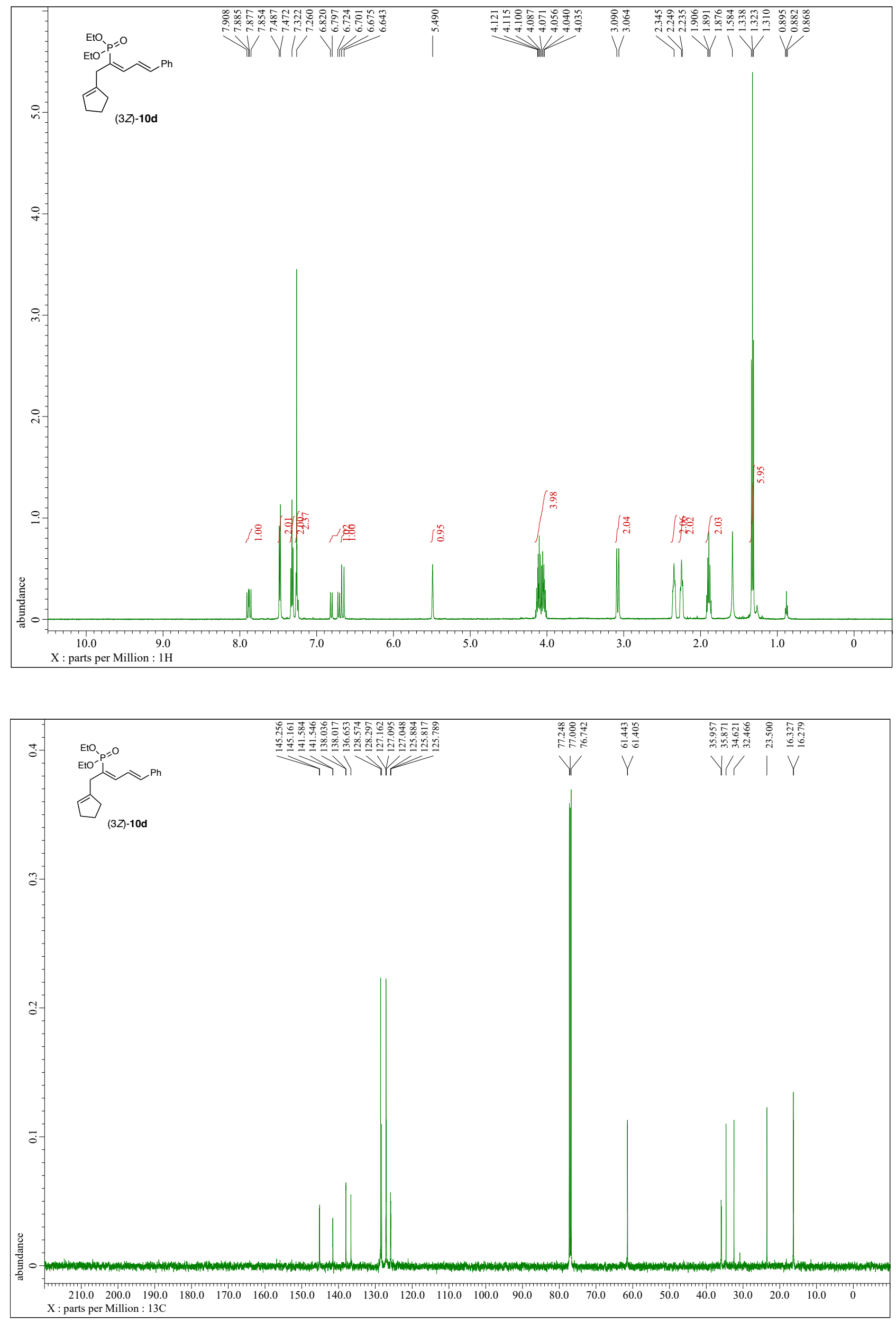


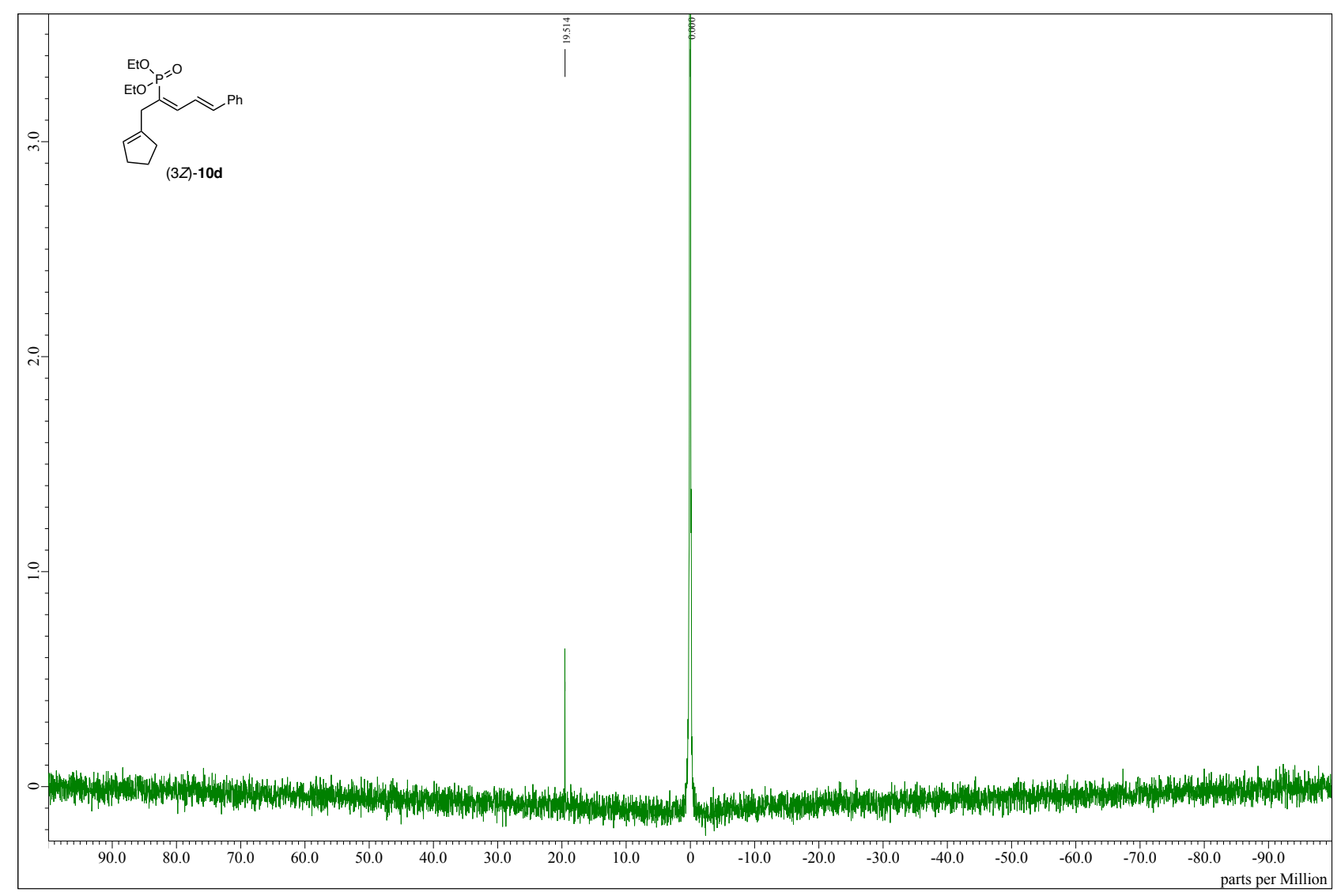


NMR spectra of (3Z)-10e $\left[{ }^{1} \mathrm{H}(500 \mathrm{MHz}),{ }^{13} \mathrm{C}(126 \mathrm{MHz})\right.$, and ${ }^{31} \mathrm{P}(202 \mathrm{MHz})$ in $\left.\mathrm{CDCl}_{3}\right]$
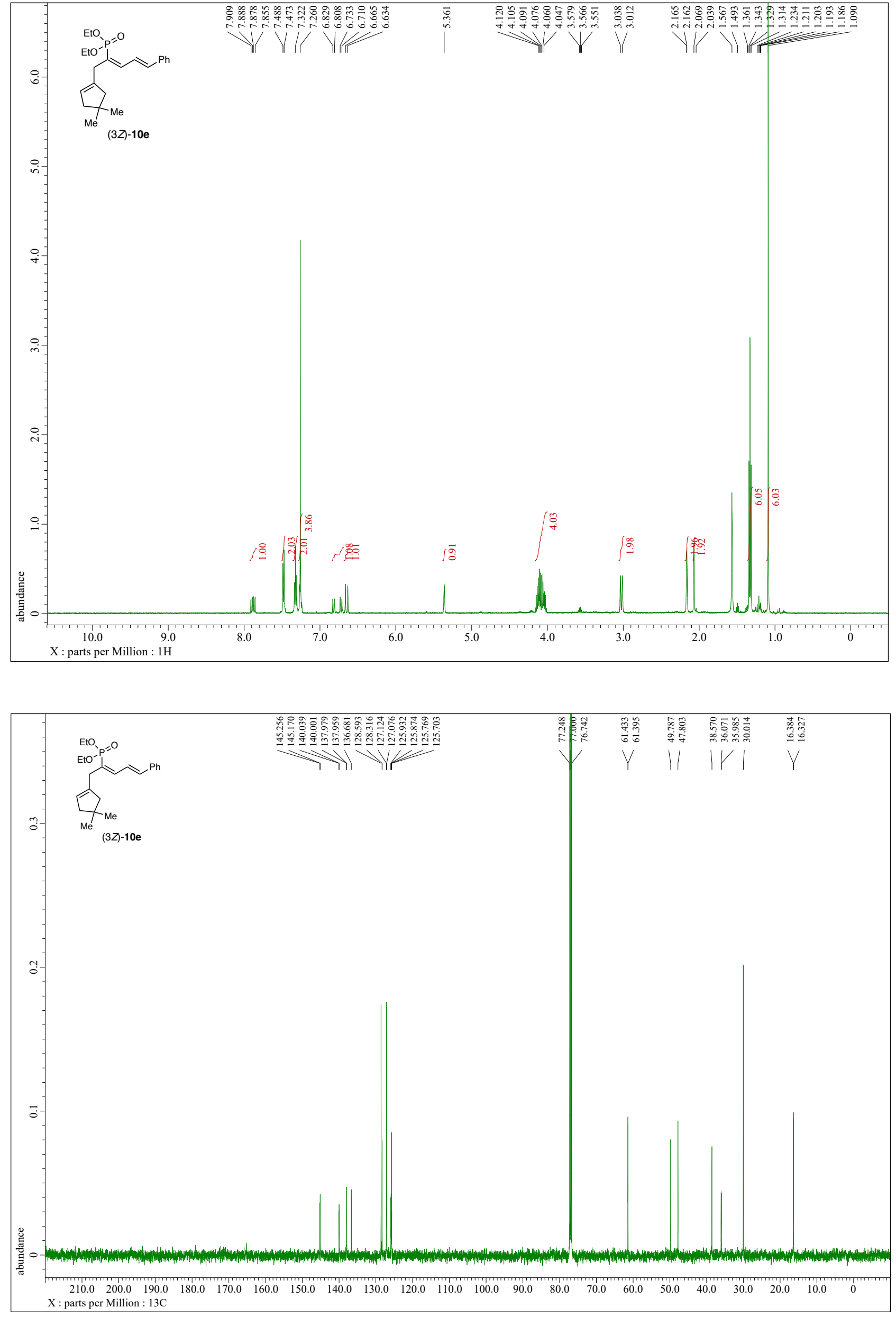


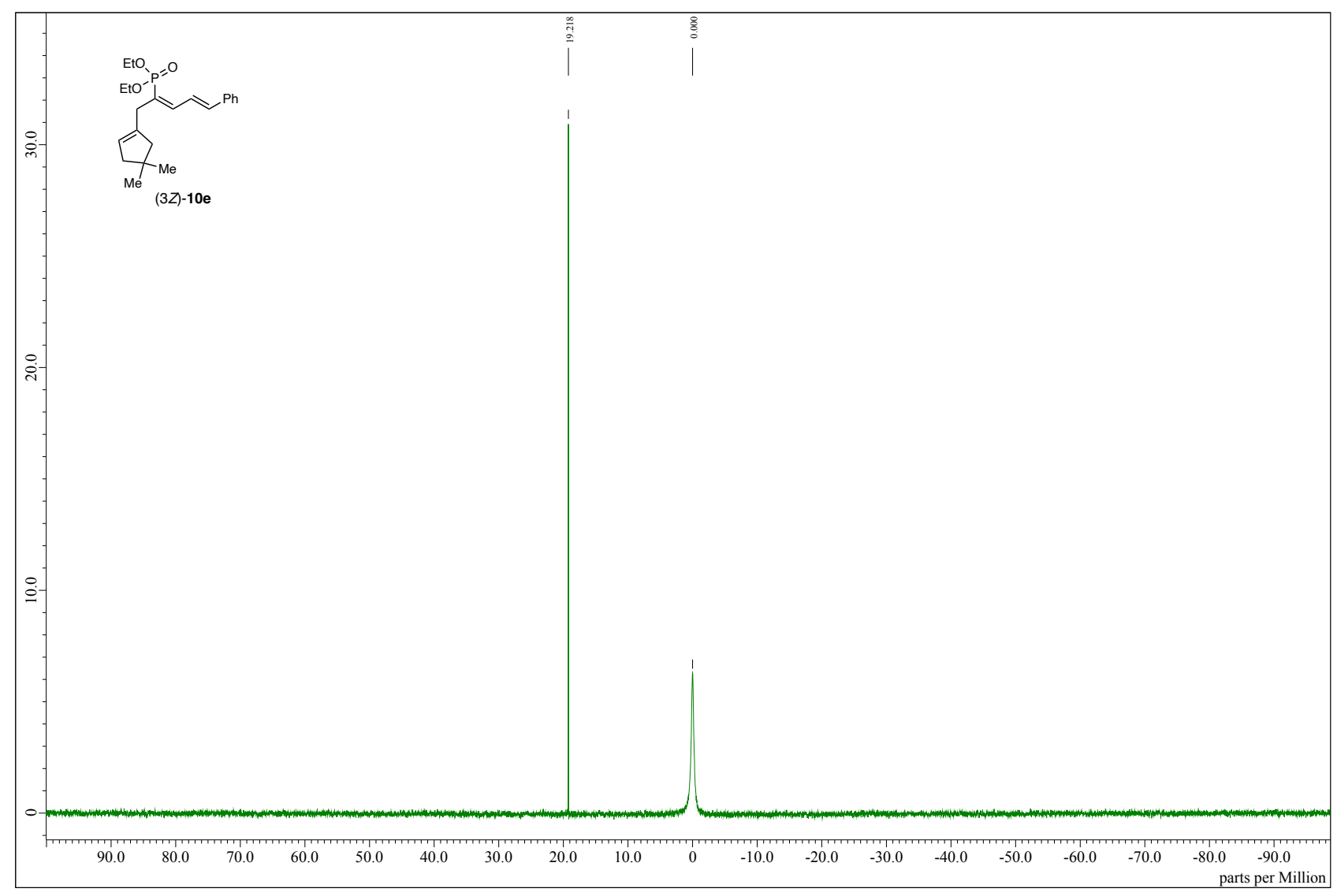


NMR spectra of (3Z)-10f $\left[{ }^{1} \mathrm{H}(500 \mathrm{MHz}),{ }^{13} \mathrm{C}(126 \mathrm{MHz})\right.$, and ${ }^{31} \mathrm{P}(202 \mathrm{MHz})$ in $\left.\mathrm{CDCl}_{3}\right]$
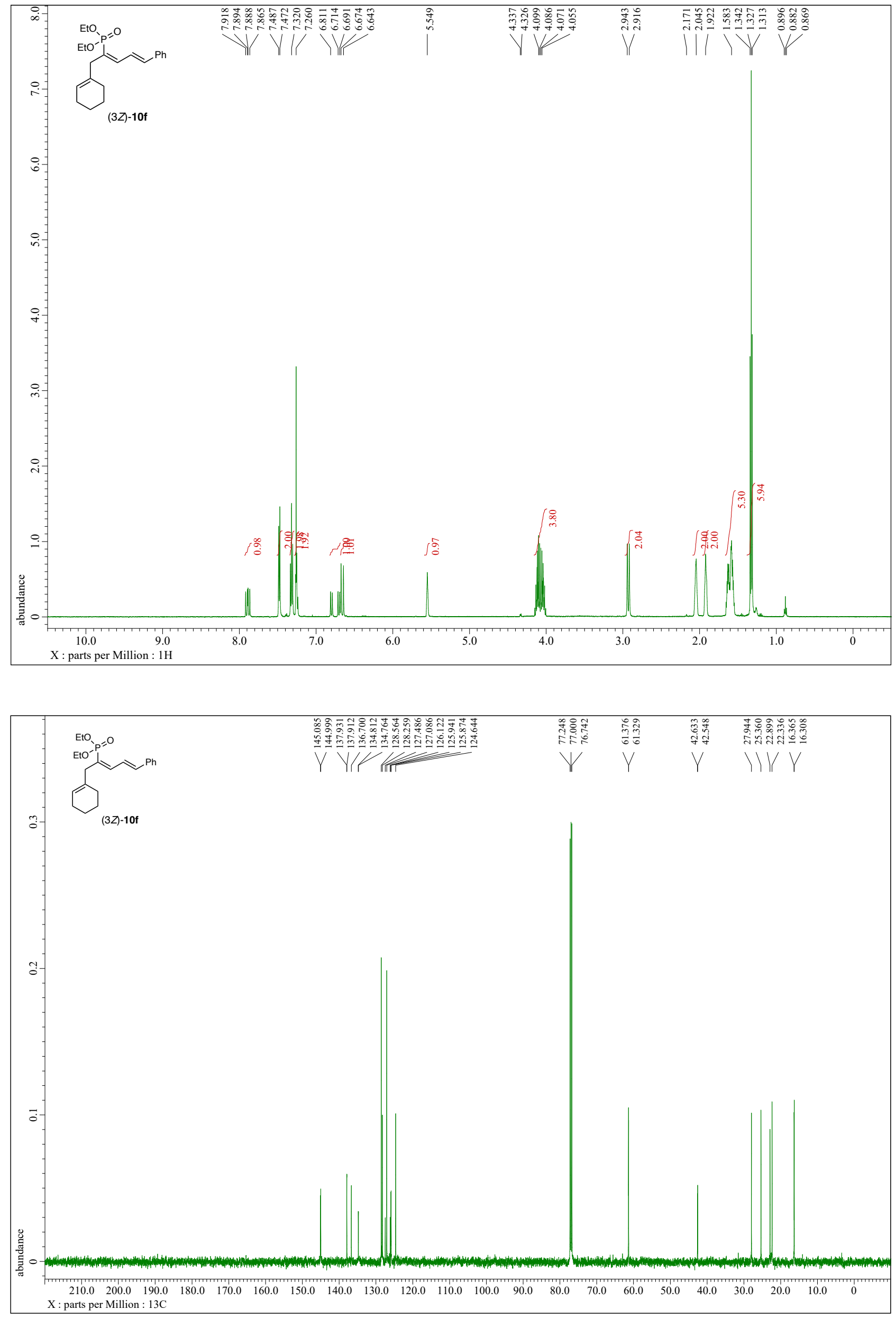


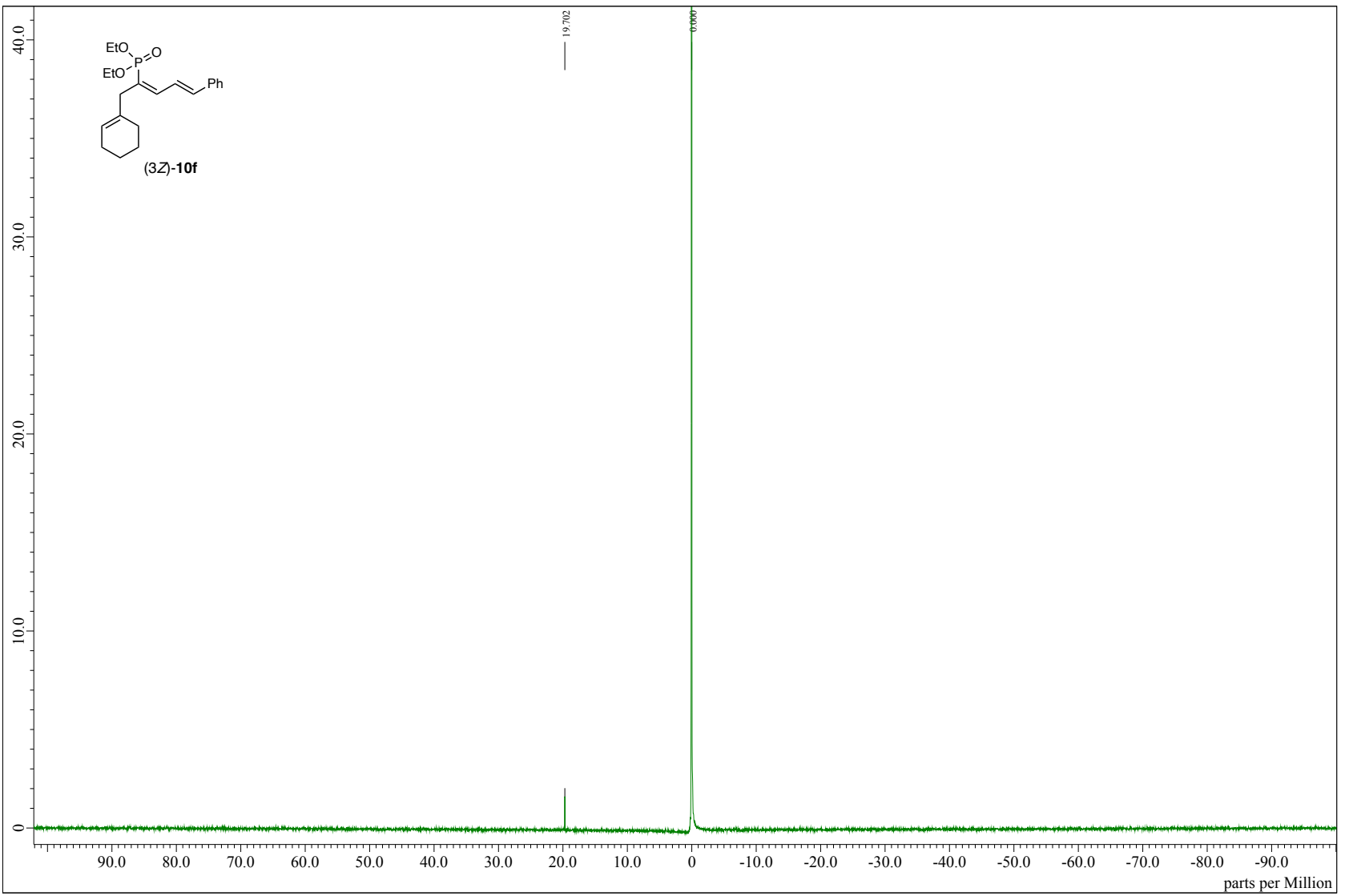


NMR spectra of (3E)-12a $\left[{ }^{1} \mathrm{H}(500 \mathrm{MHz}),{ }^{13} \mathrm{C}(126 \mathrm{MHz})\right.$, and ${ }^{31} \mathrm{P}(202 \mathrm{MHz})$ in $\left.\mathrm{CDCl}_{3}\right]$
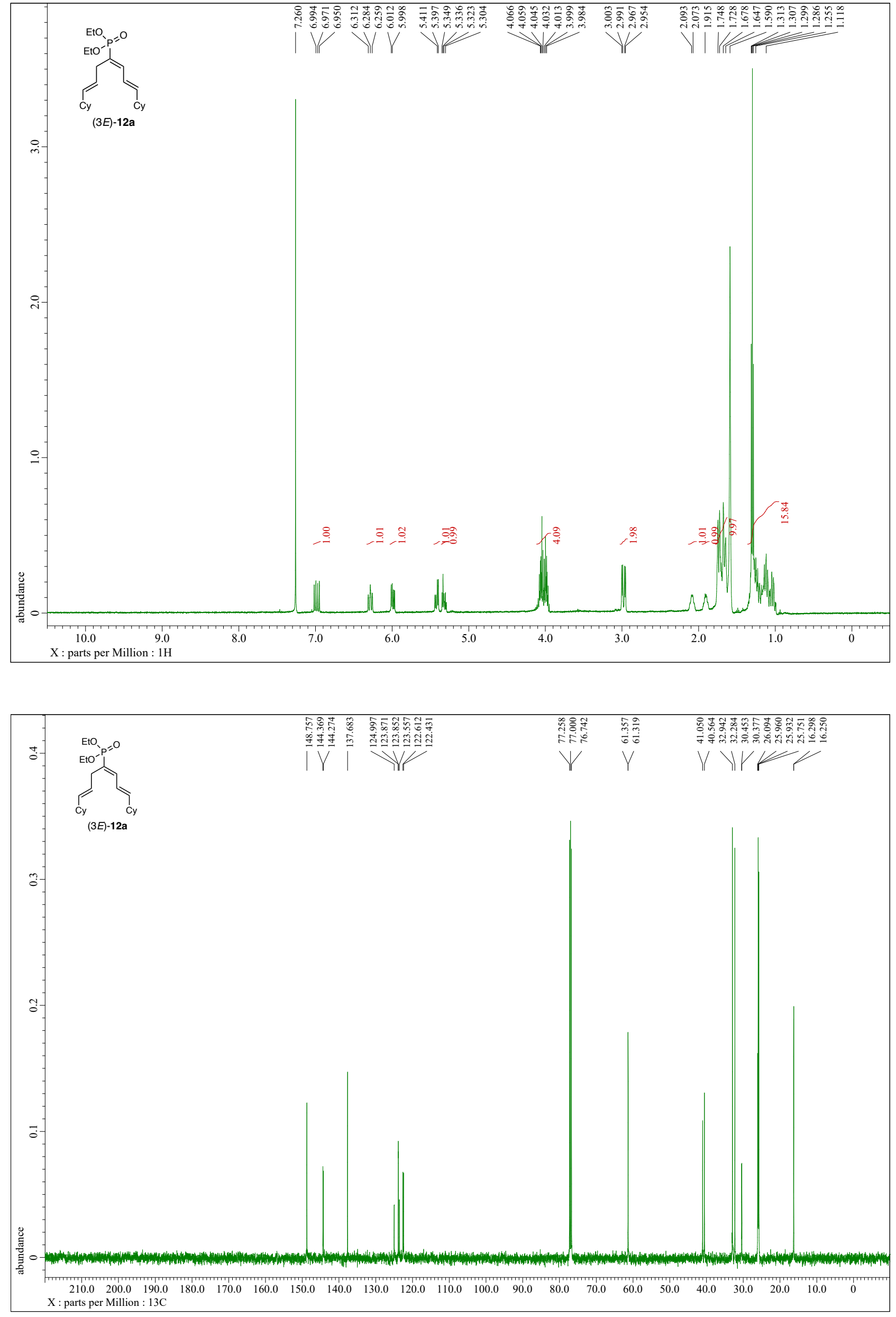


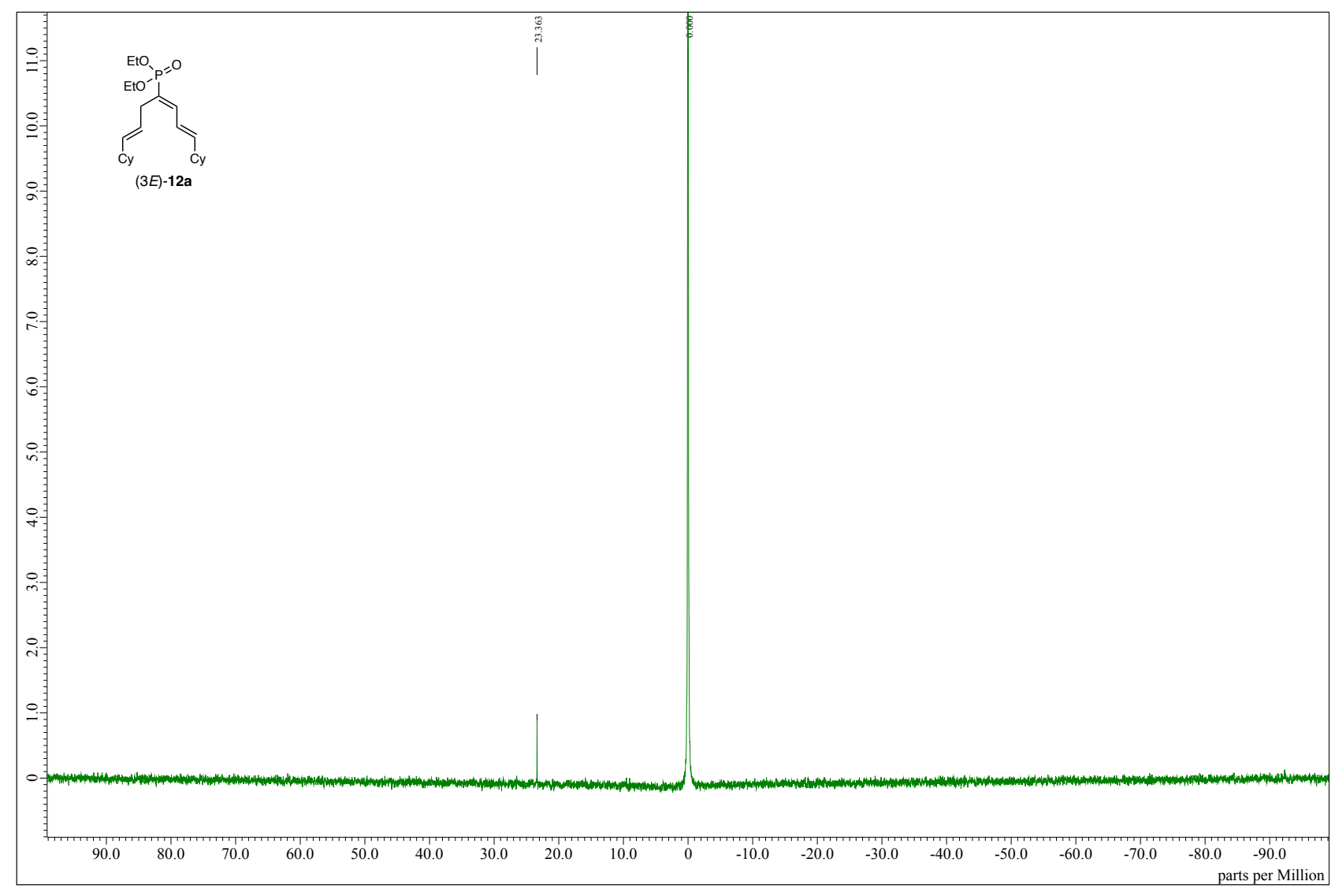


NMR spectra of (3Z)-12a $\left[{ }^{1} \mathrm{H}(500 \mathrm{MHz}),{ }^{13} \mathrm{C}(126 \mathrm{MHz})\right.$, and ${ }^{31} \mathrm{P}(202 \mathrm{MHz})$ in $\left.\mathrm{CDCl}_{3}\right]$
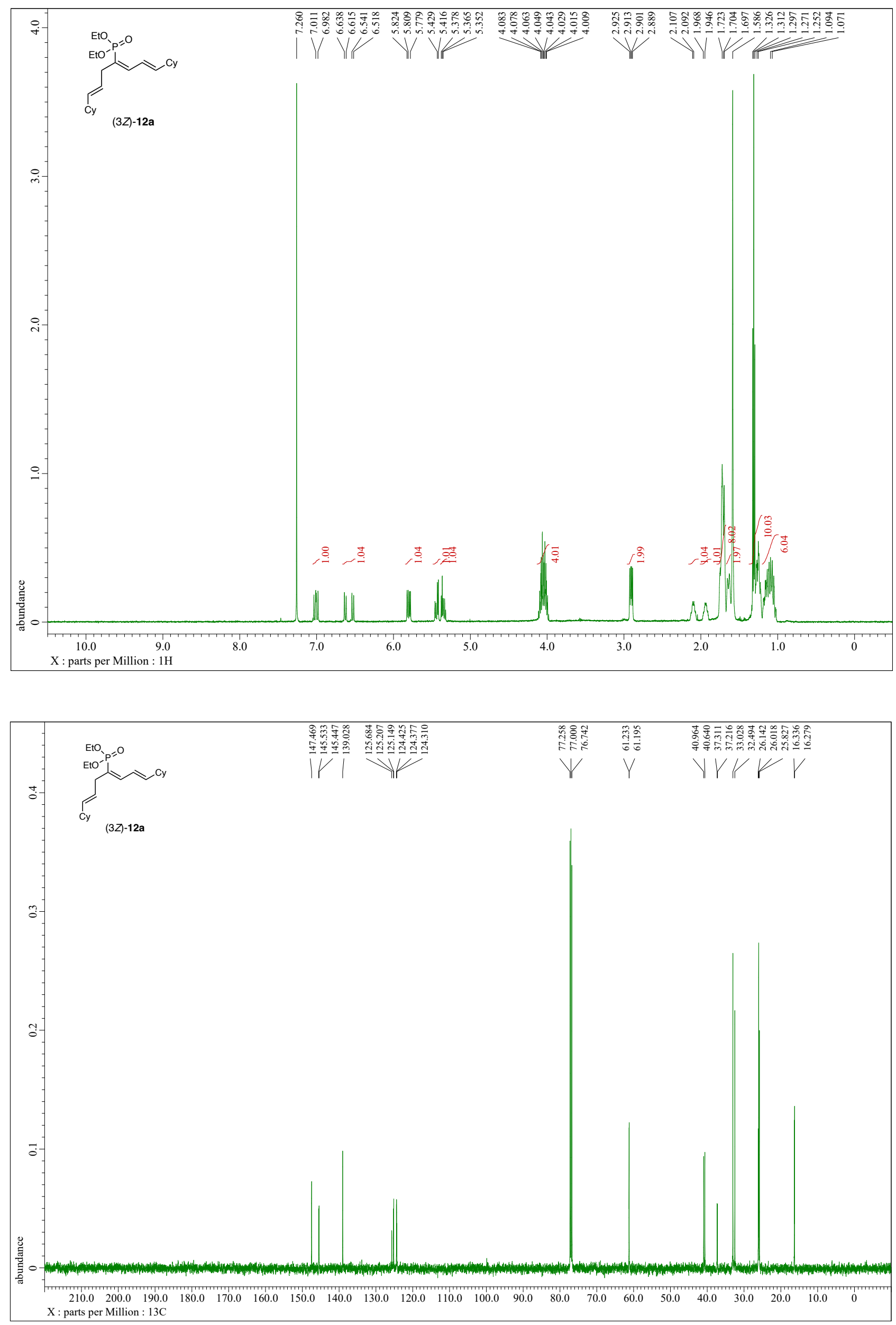


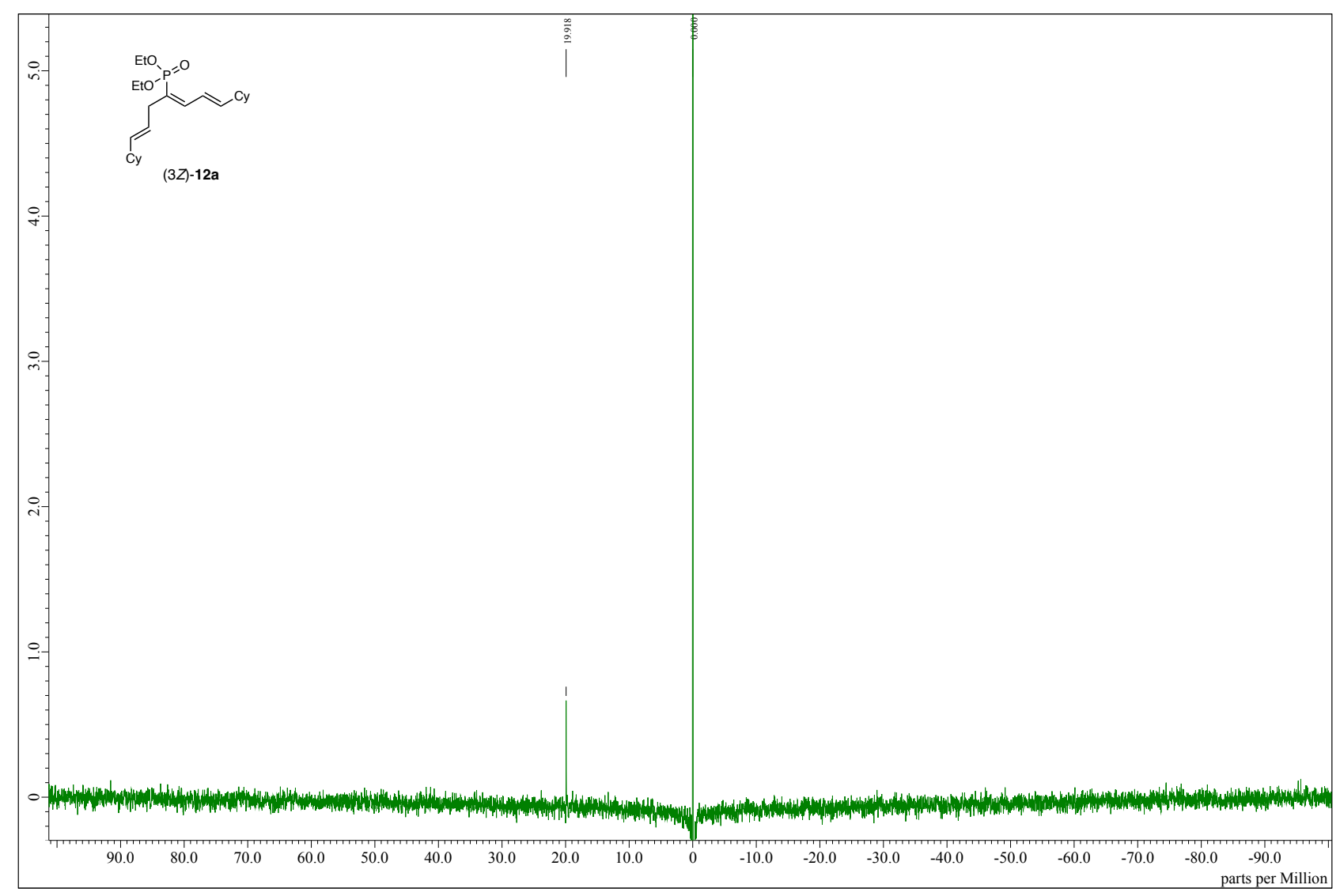


NMR spectra of $(3 E)-\mathbf{1 2 b}\left[{ }^{1} \mathrm{H}(500 \mathrm{MHz}),{ }^{13} \mathrm{C}(126 \mathrm{MHz})\right.$, and ${ }^{31} \mathrm{P}(202 \mathrm{MHz})$ in $\left.\mathrm{CDCl}_{3}\right]$
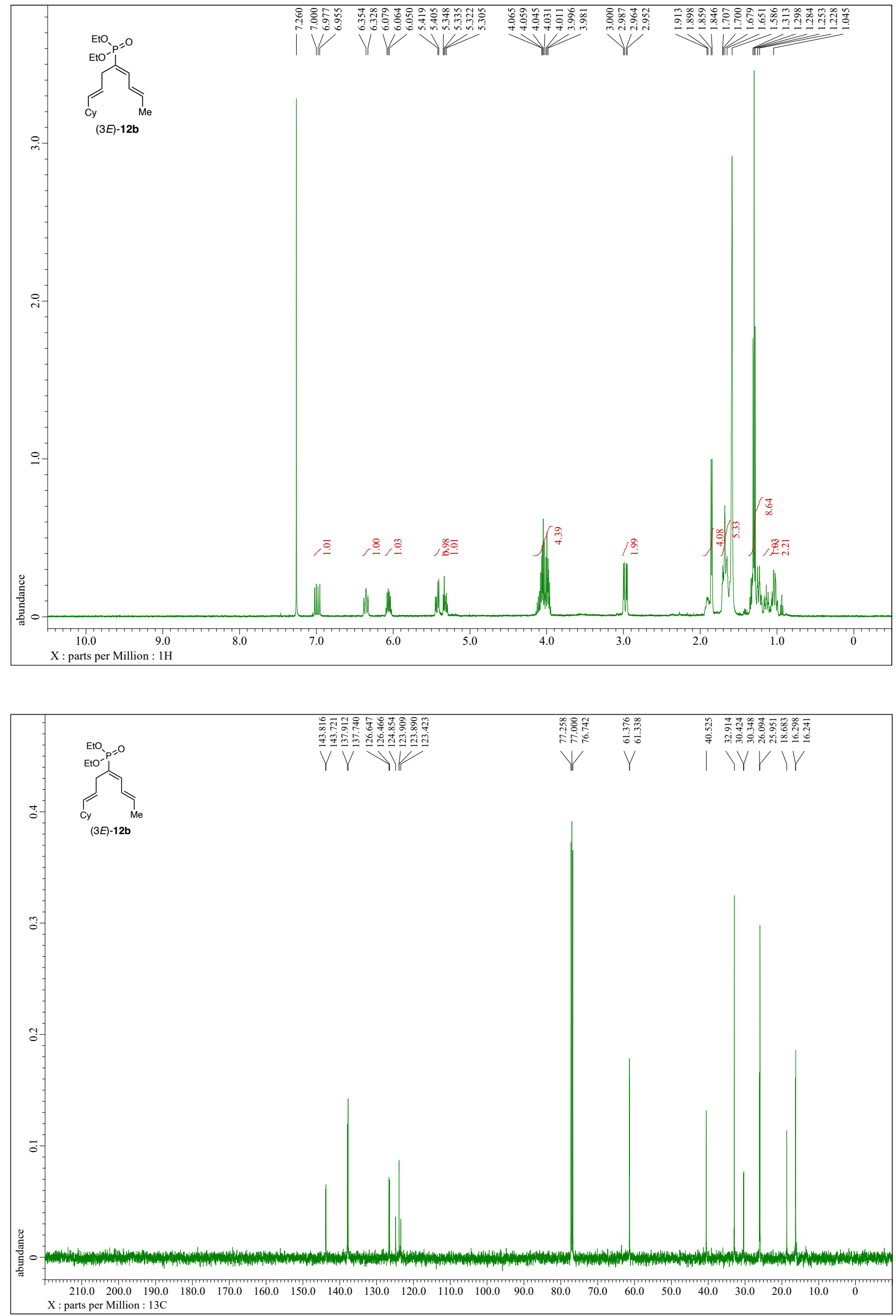


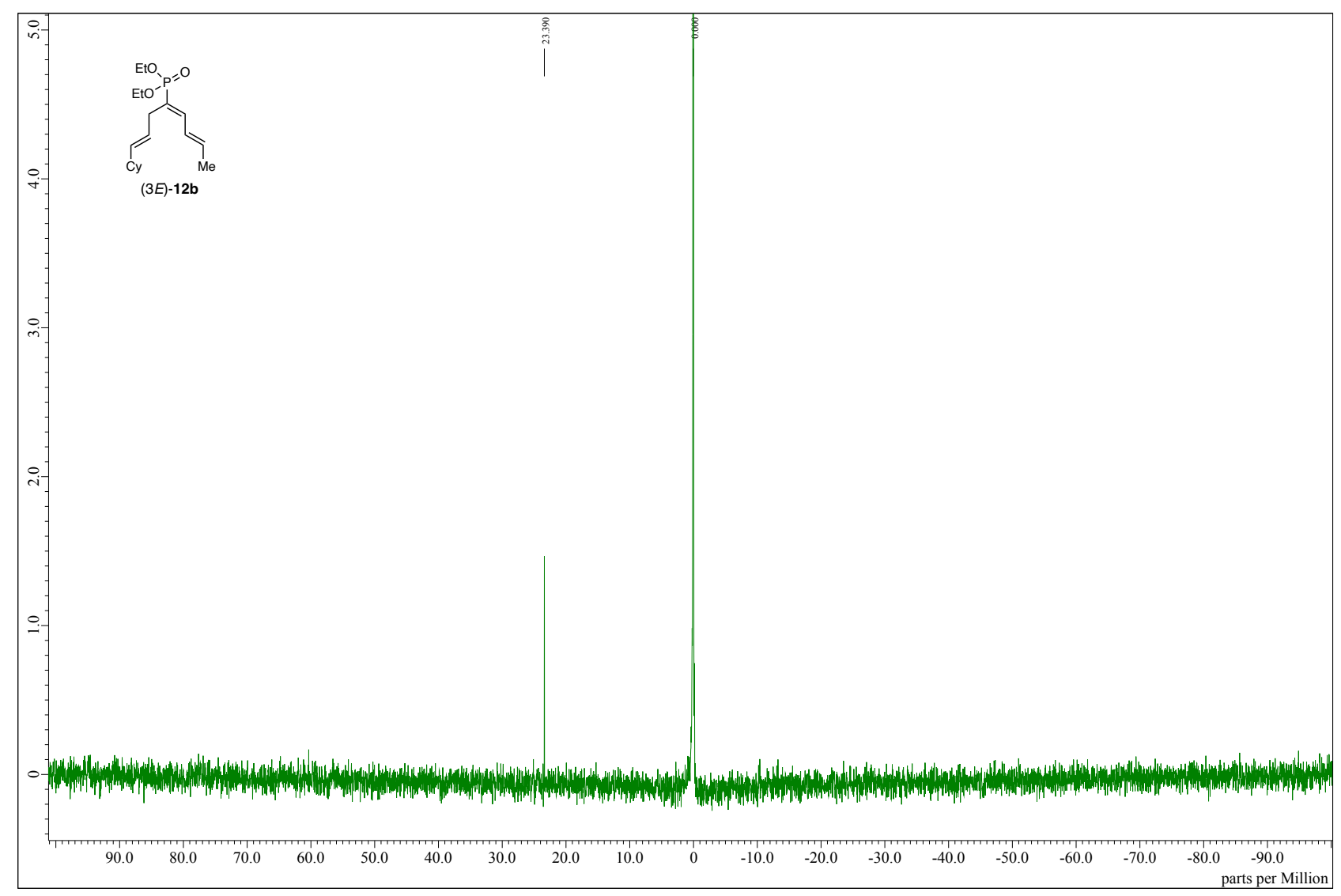


NMR spectra of (3Z)-12b $\left[{ }^{1} \mathrm{H}(500 \mathrm{MHz}),{ }^{13} \mathrm{C}(126 \mathrm{MHz})\right.$, and ${ }^{31} \mathrm{P}(202 \mathrm{MHz})$ in $\left.\mathrm{CDCl}_{3}\right]$
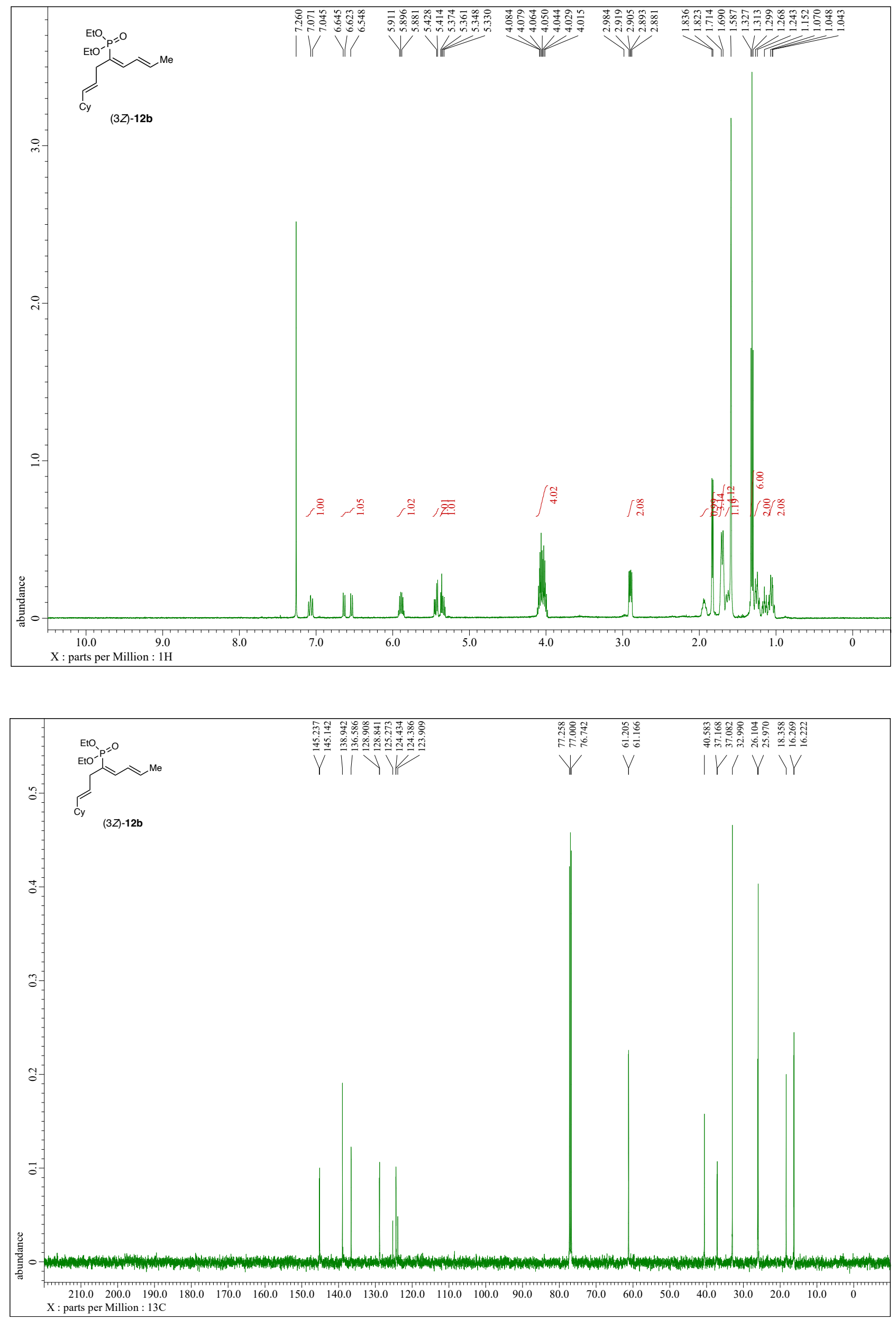


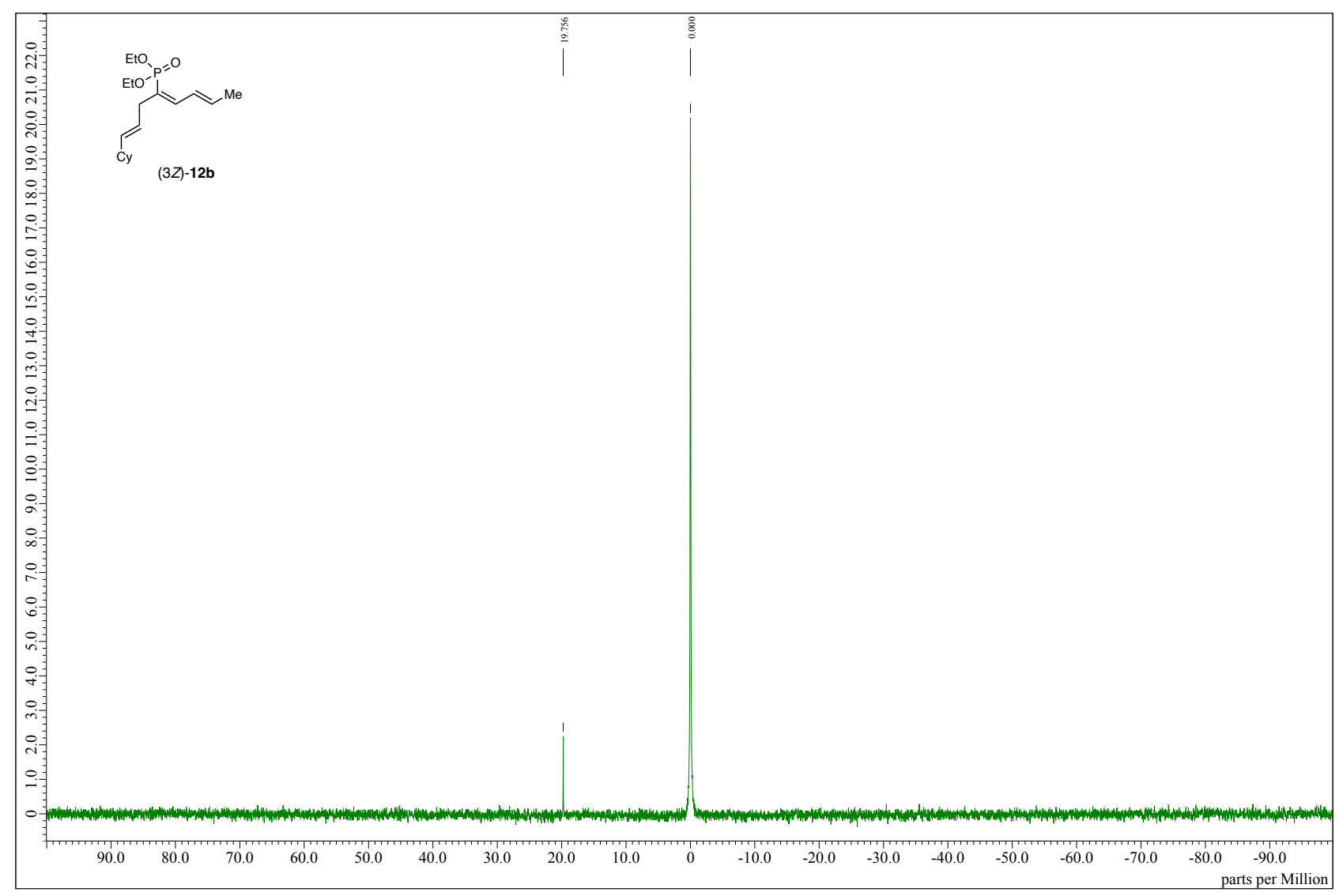


NMR spectra of (3E)-12c $\left[{ }^{1} \mathrm{H}(500 \mathrm{MHz}),{ }^{13} \mathrm{C}(126 \mathrm{MHz})\right.$, and ${ }^{31} \mathrm{P}(202 \mathrm{MHz})$ in $\left.\mathrm{CDCl}_{3}\right]$
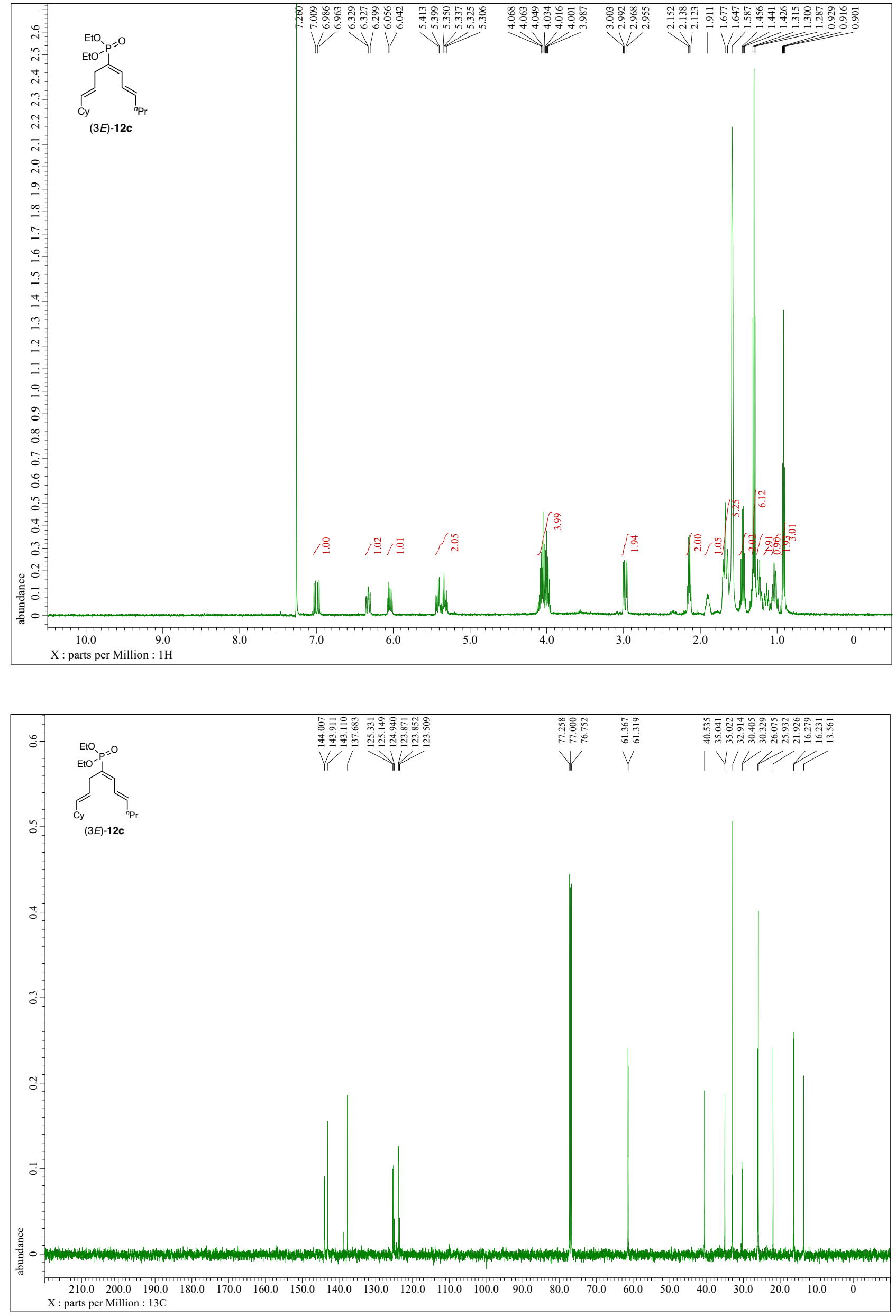


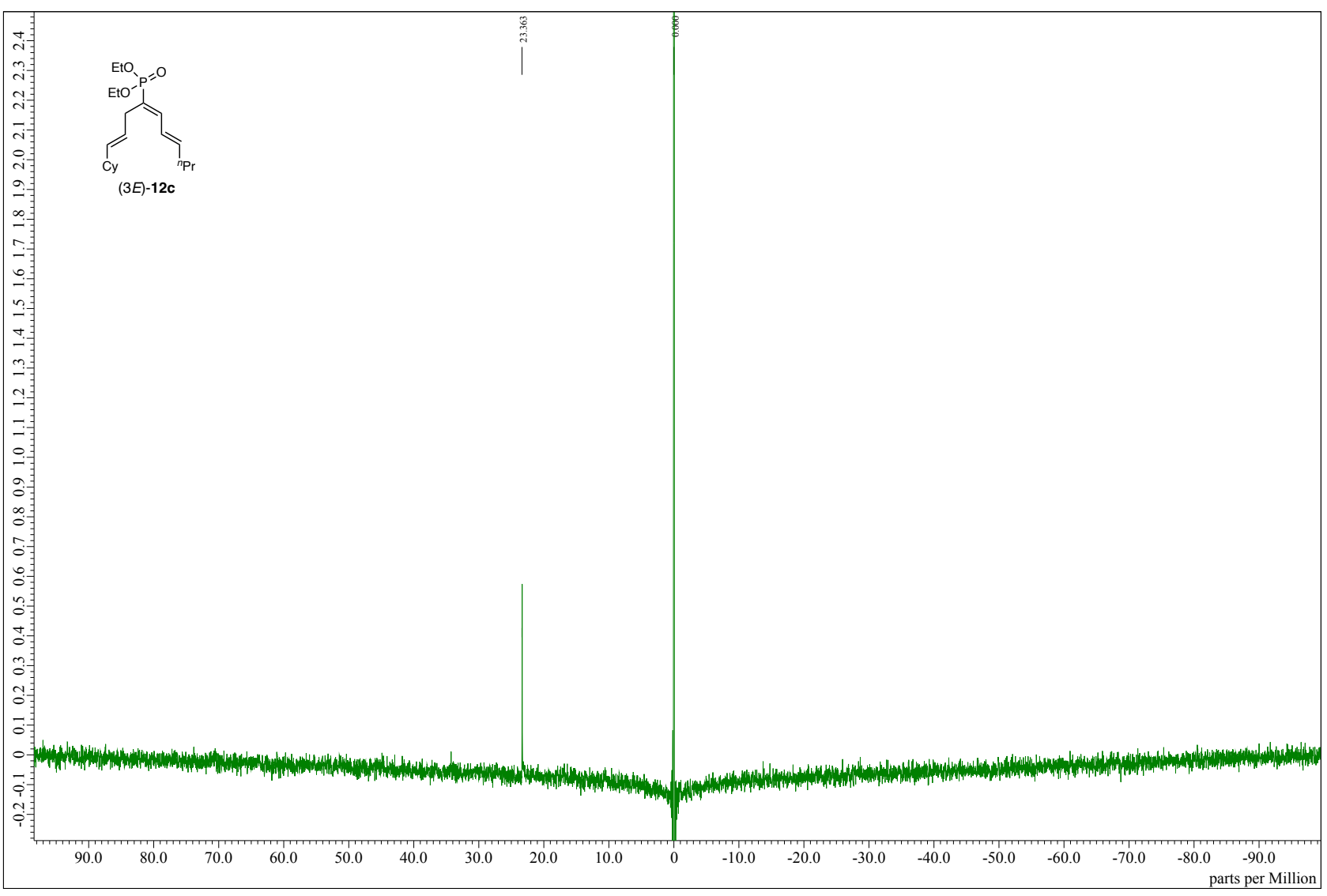


NMR spectra of (3Z)-12c [ ${ }^{1} \mathrm{H}(500 \mathrm{MHz}),{ }^{13} \mathrm{C}(126 \mathrm{MHz})$, and ${ }^{31} \mathrm{P}(202 \mathrm{MHz})$ in $\left.\mathrm{CDCl}_{3}\right]$
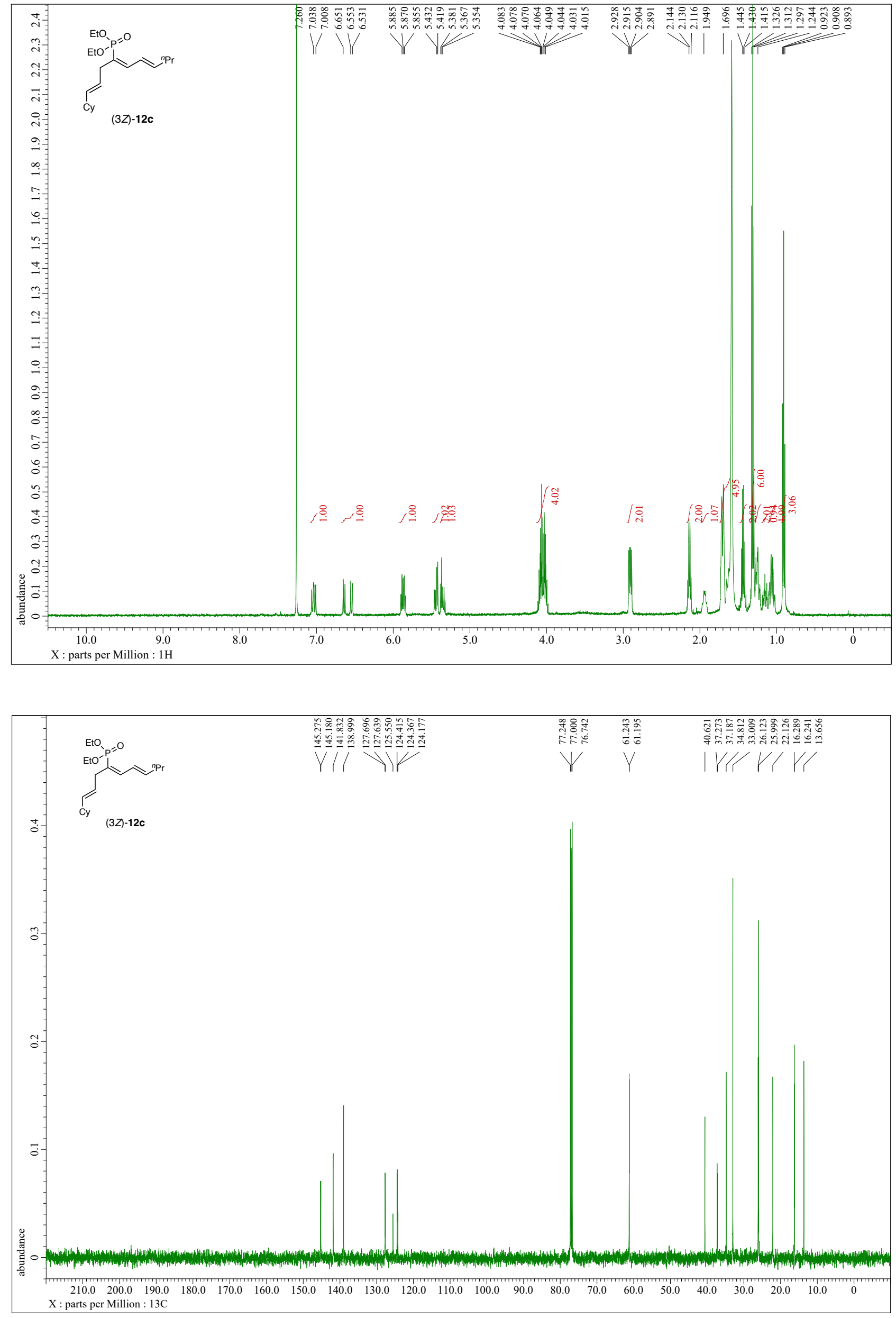


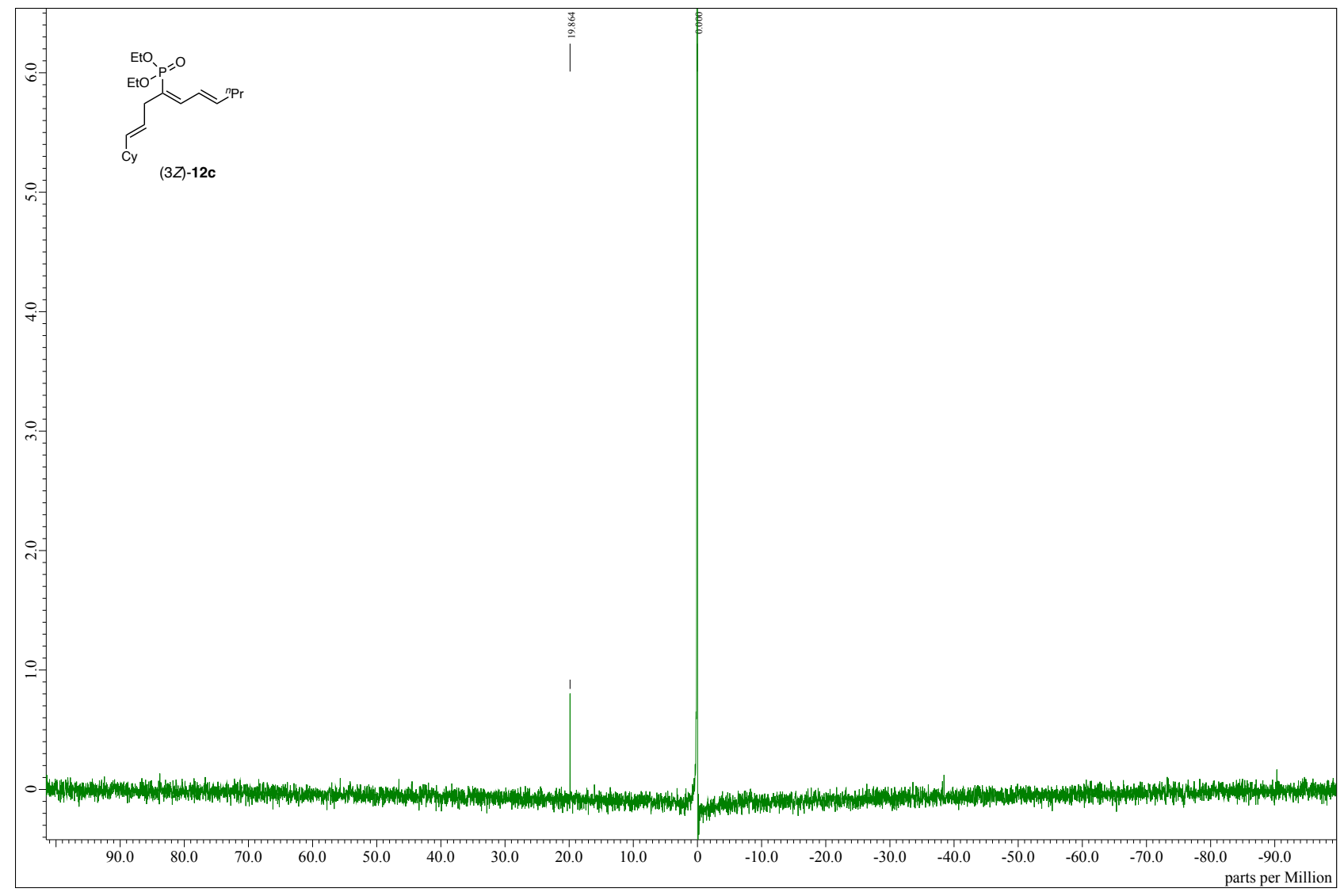


NMR spectra of (3E)-12d $\left[{ }^{1} \mathrm{H}(500 \mathrm{MHz}),{ }^{13} \mathrm{C}(126 \mathrm{MHz})\right.$, and ${ }^{31} \mathrm{P}(202 \mathrm{MHz})$ in $\left.\mathrm{CDCl}_{3}\right]$
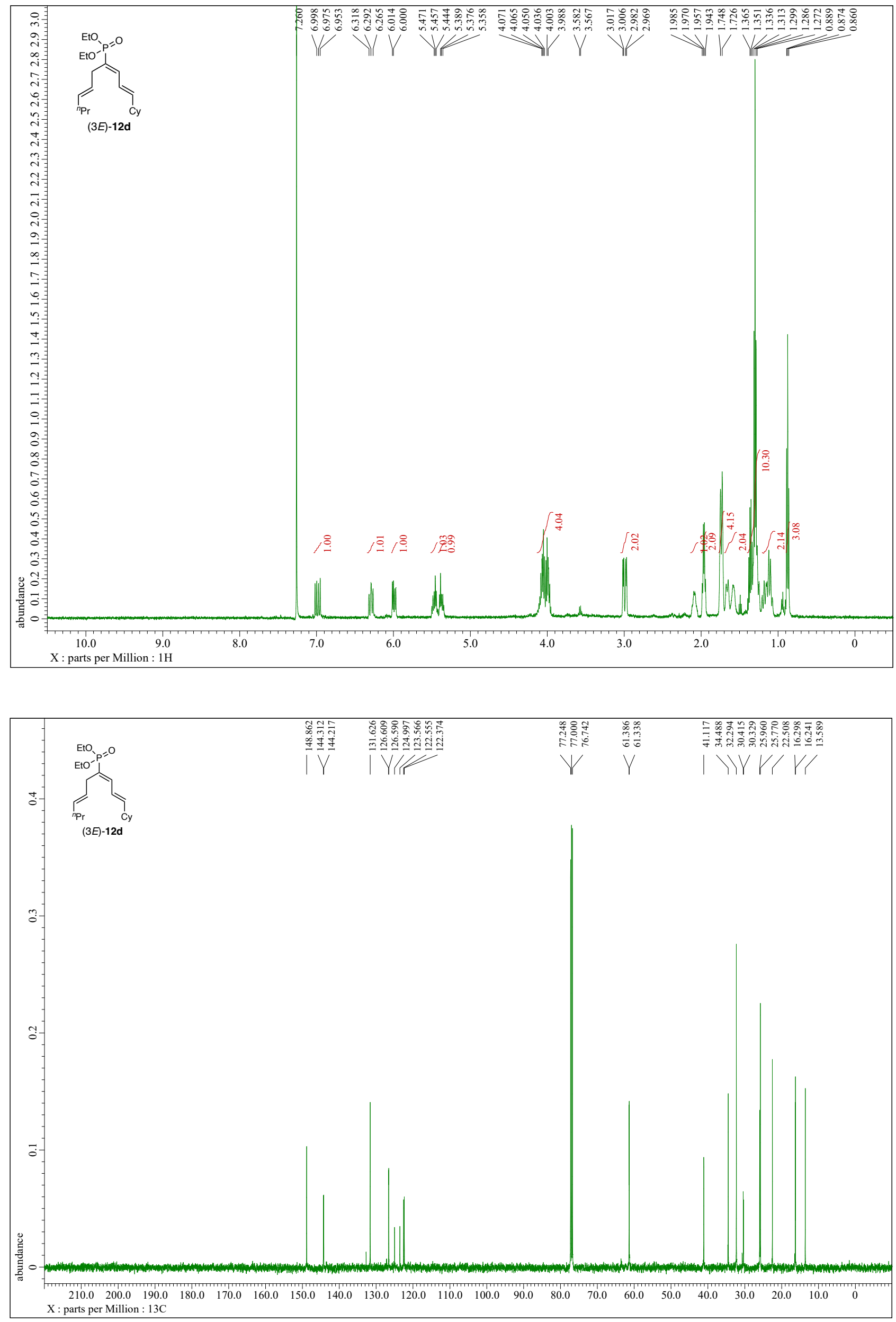


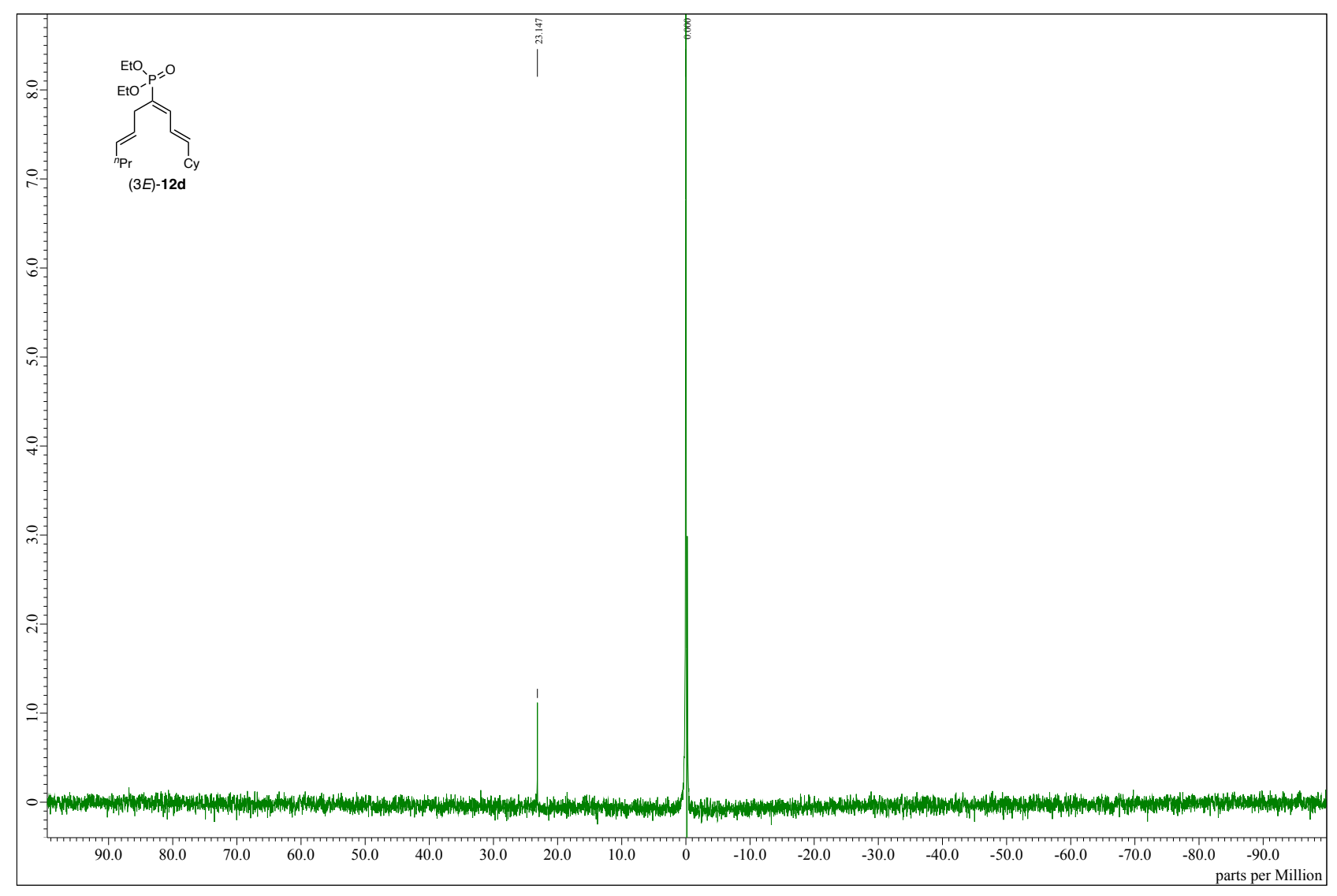


NMR spectra of (3Z)-12d $\left[{ }^{1} \mathrm{H}(500 \mathrm{MHz}),{ }^{13} \mathrm{C}(126 \mathrm{MHz})\right.$, and ${ }^{31} \mathrm{P}(202 \mathrm{MHz})$ in $\left.\mathrm{CDCl}_{3}\right]$
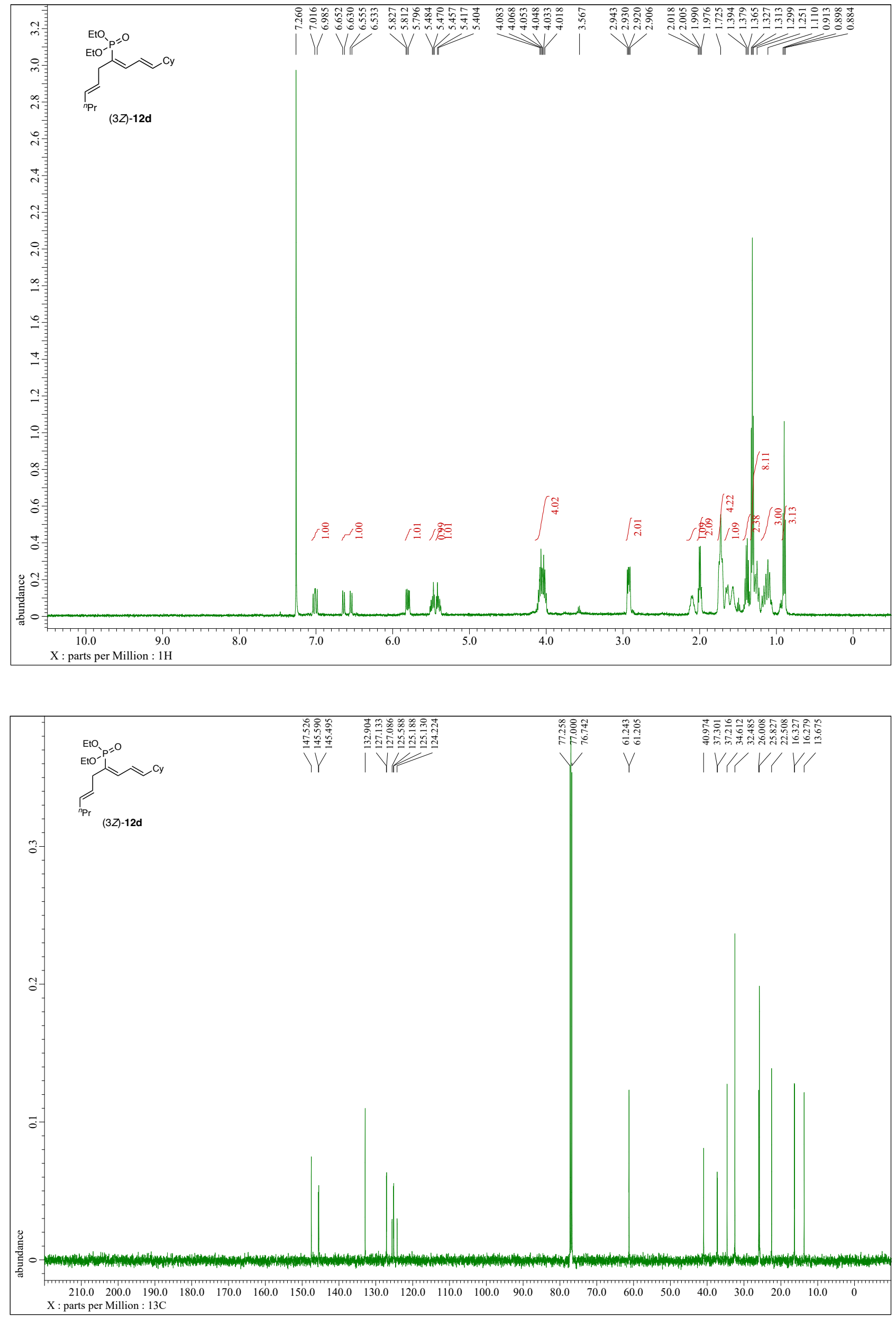


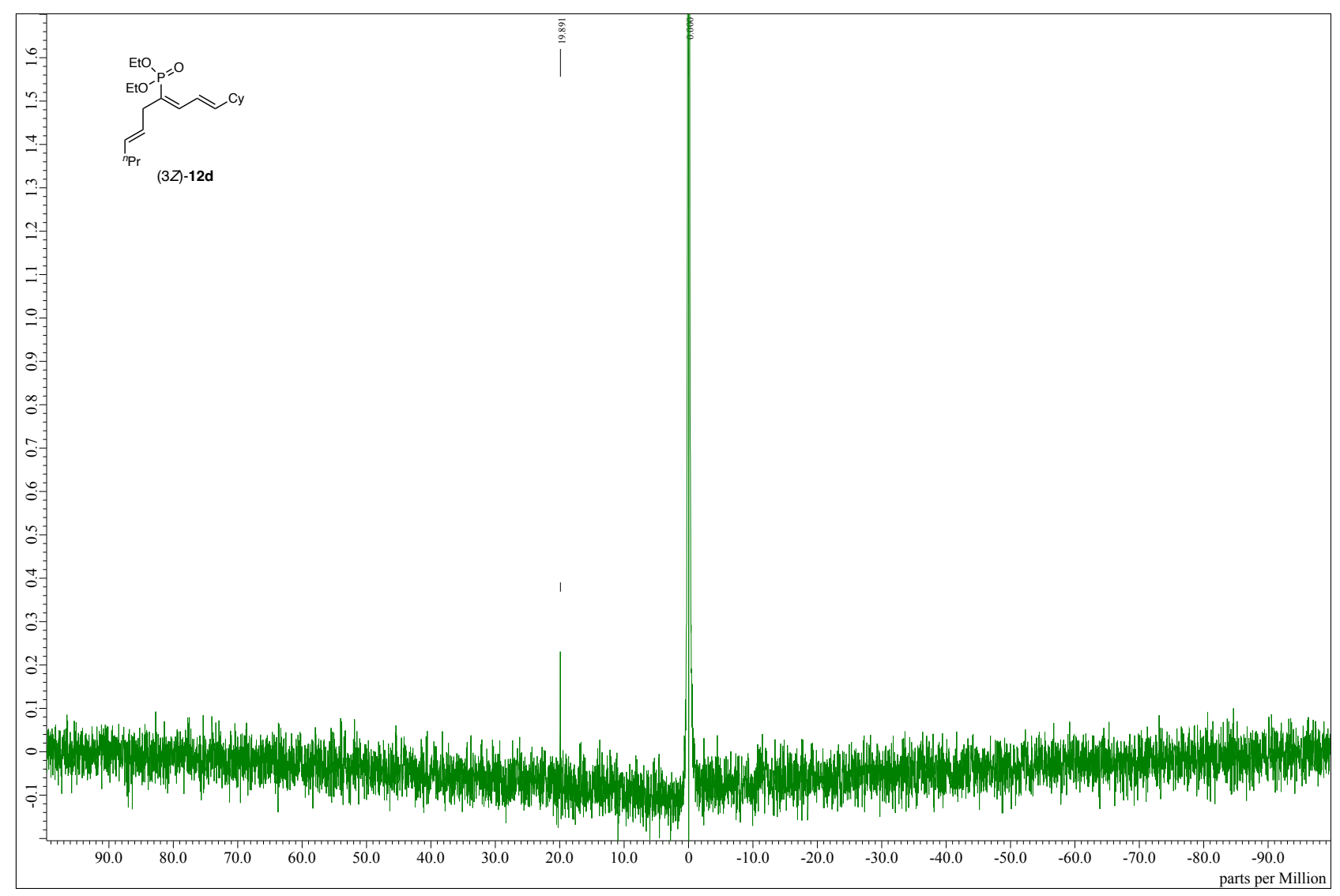


NMR spectra of $6 \mathbf{a}\left[{ }^{1} \mathrm{H}(500 \mathrm{MHz}),{ }^{13} \mathrm{C}(126 \mathrm{MHz})\right.$, and ${ }^{31} \mathrm{P}(202 \mathrm{MHz})$ in $\left.\mathrm{CDCl}_{3}\right]$
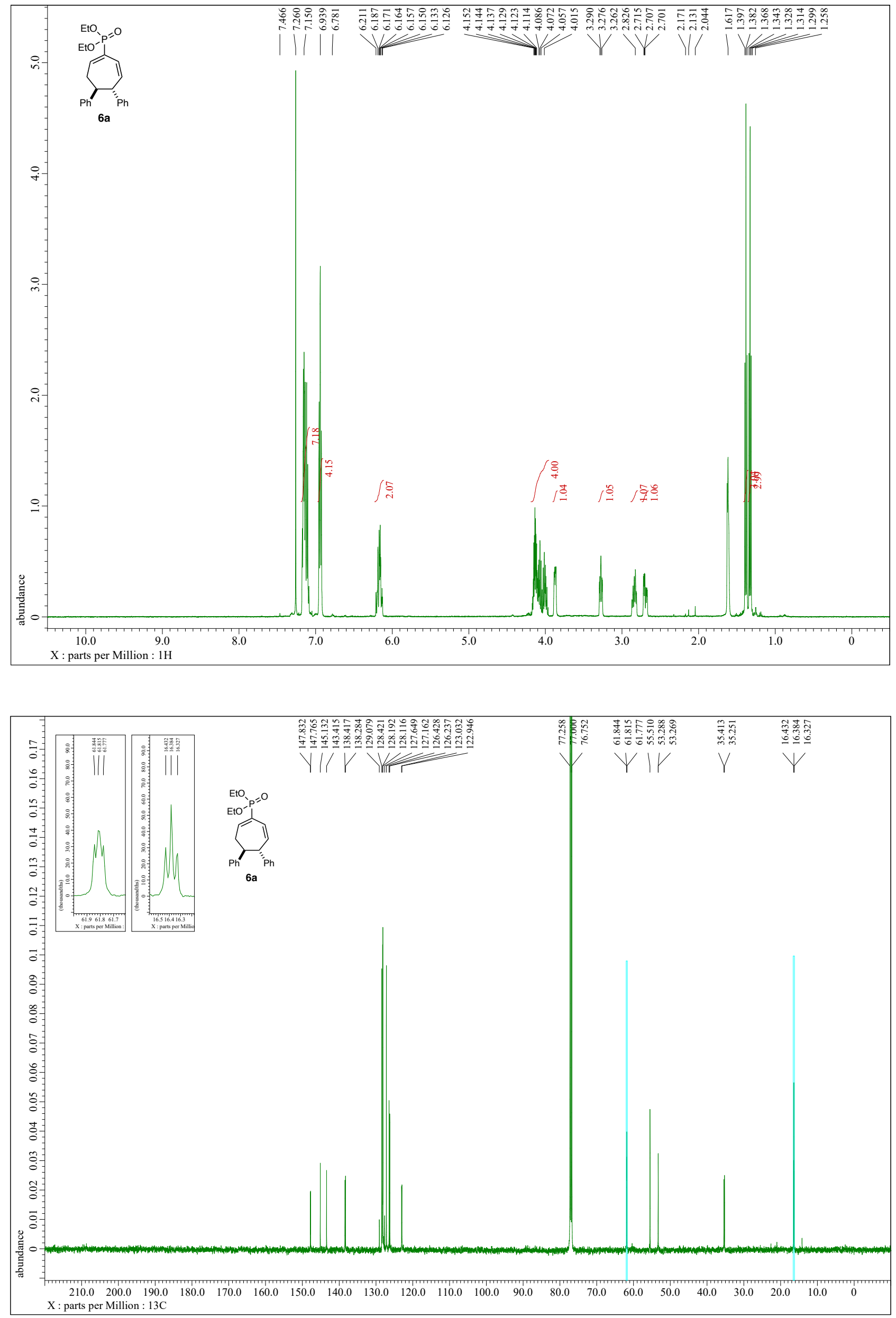


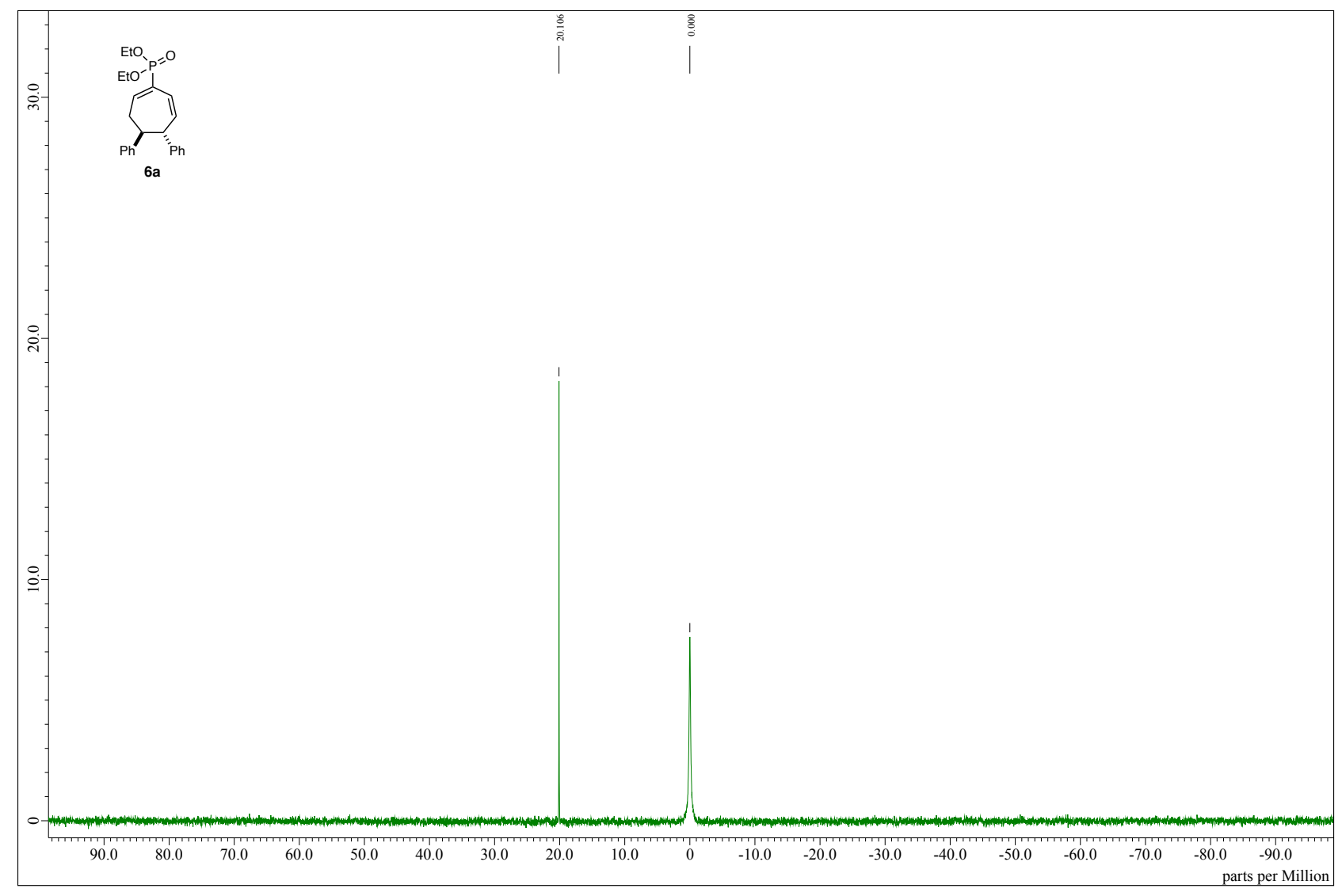


NMR spectra of $\mathbf{8 a}$ and $\mathbf{8 b}\left[{ }^{1} \mathrm{H}(500 \mathrm{MHz}),{ }^{13} \mathrm{C}(126 \mathrm{MHz})\right.$, and ${ }^{31} \mathrm{P}(202 \mathrm{MHz})$ in $\left.\mathrm{CDCl}_{3}\right]$
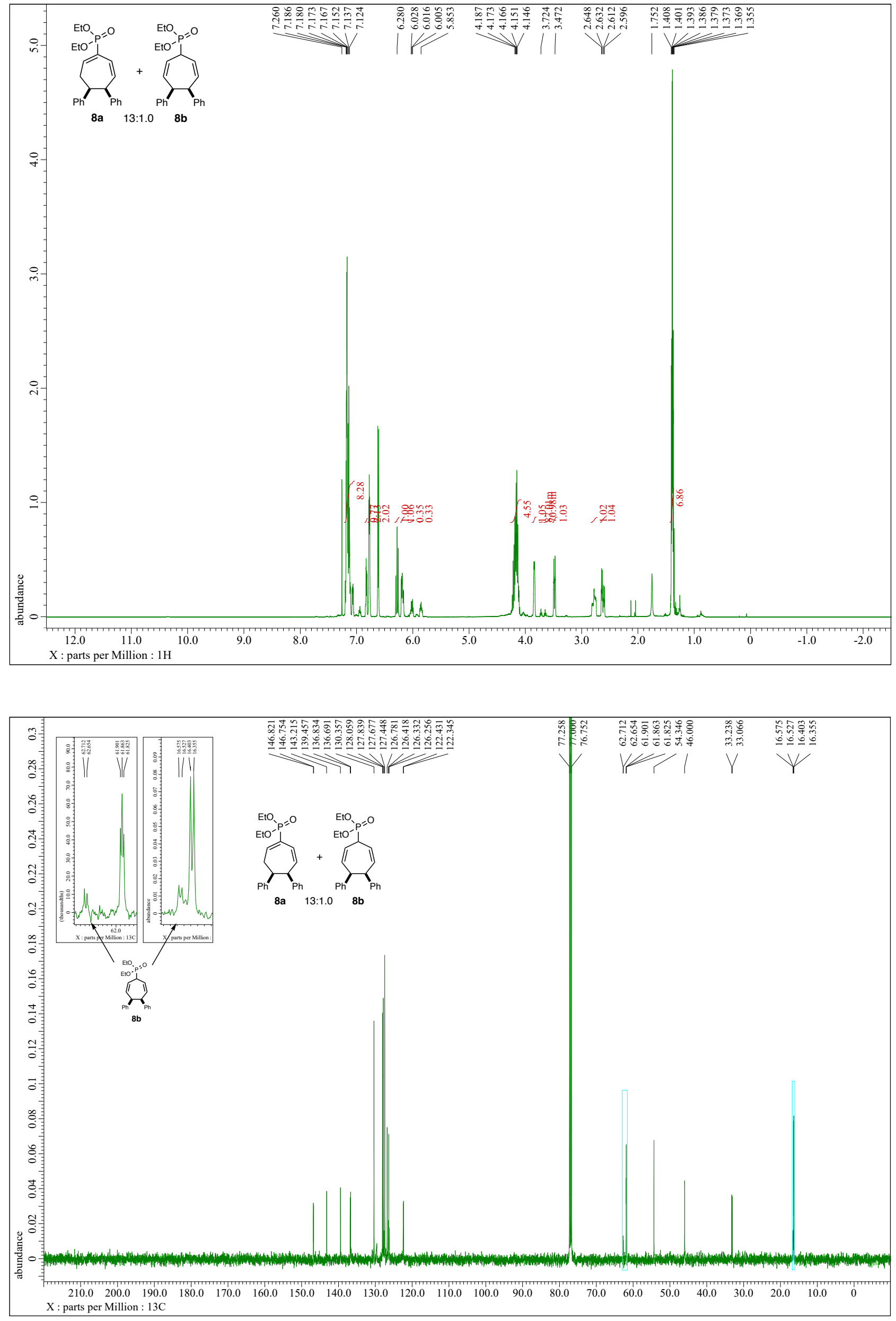


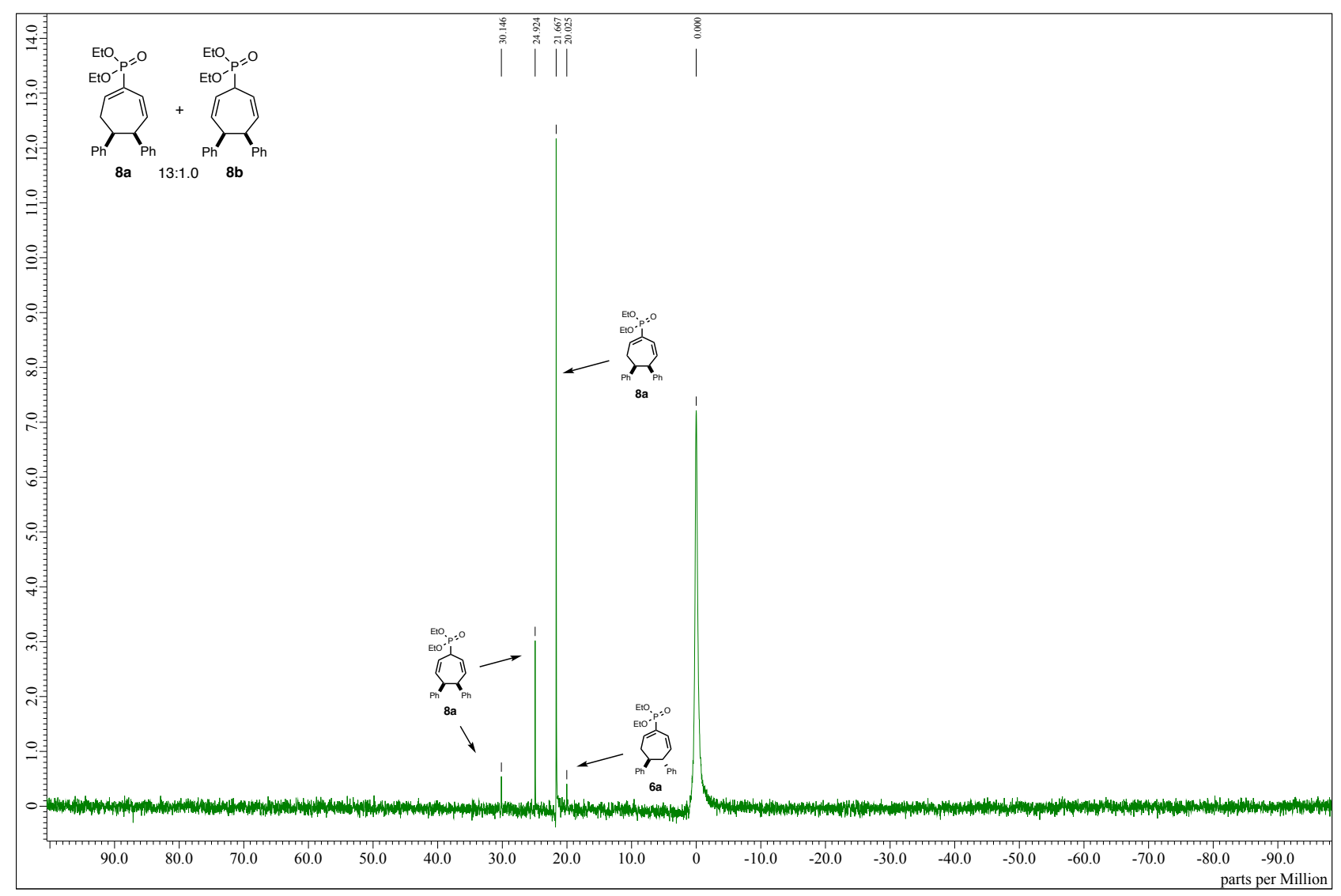


NMR spectra of $7\left[{ }^{1} \mathrm{H}(500 \mathrm{MHz})\right.$ and ${ }^{13} \mathrm{C}(126 \mathrm{MHz})$ in $\left.\mathrm{CDCl}_{3}\right]$
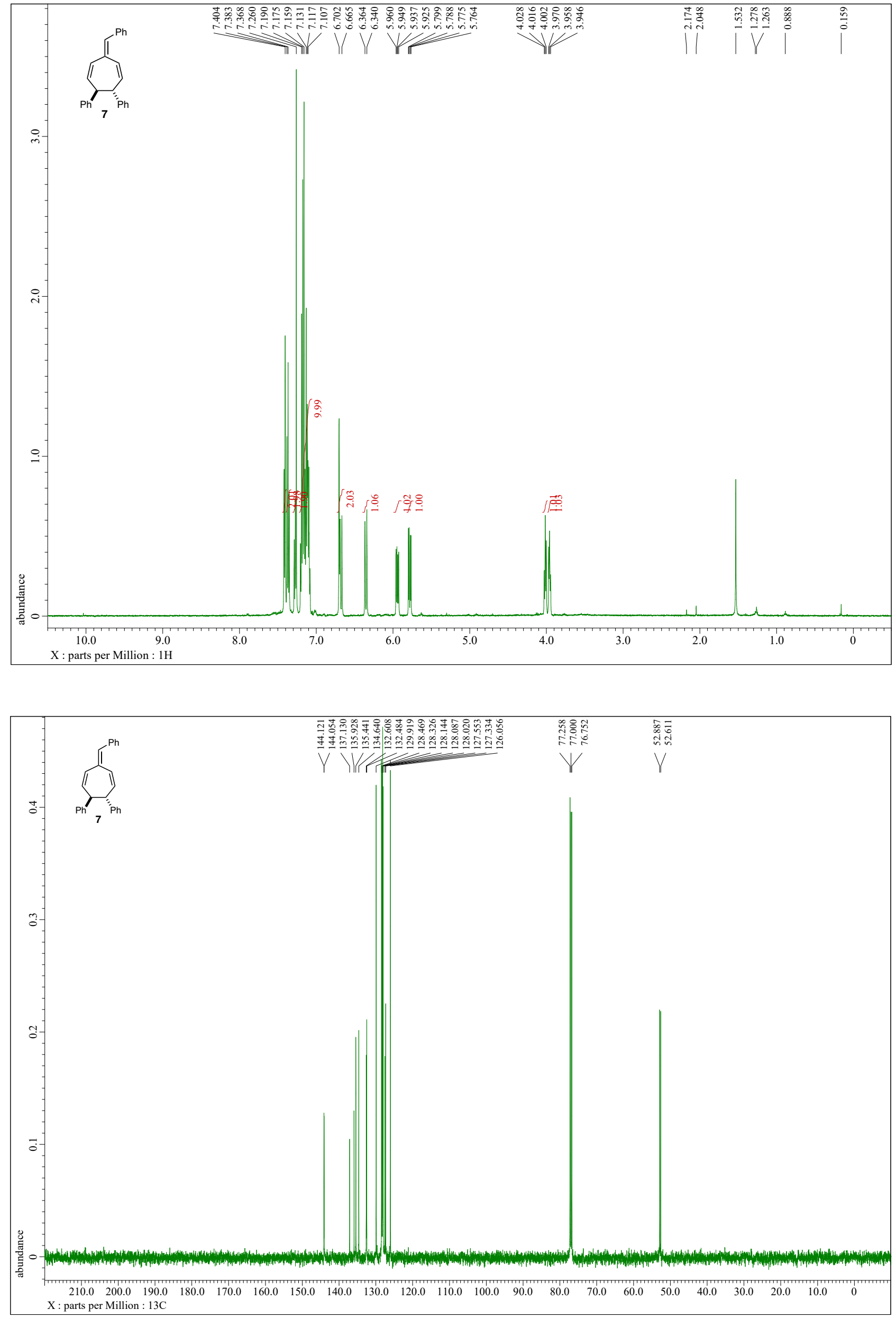
NMR spectra of 9a $\left[{ }^{1} \mathrm{H}(500 \mathrm{MHz})\right.$ and ${ }^{13} \mathrm{C}(126 \mathrm{MHz})$ in $\left.\mathrm{CDCl}_{3}\right]$
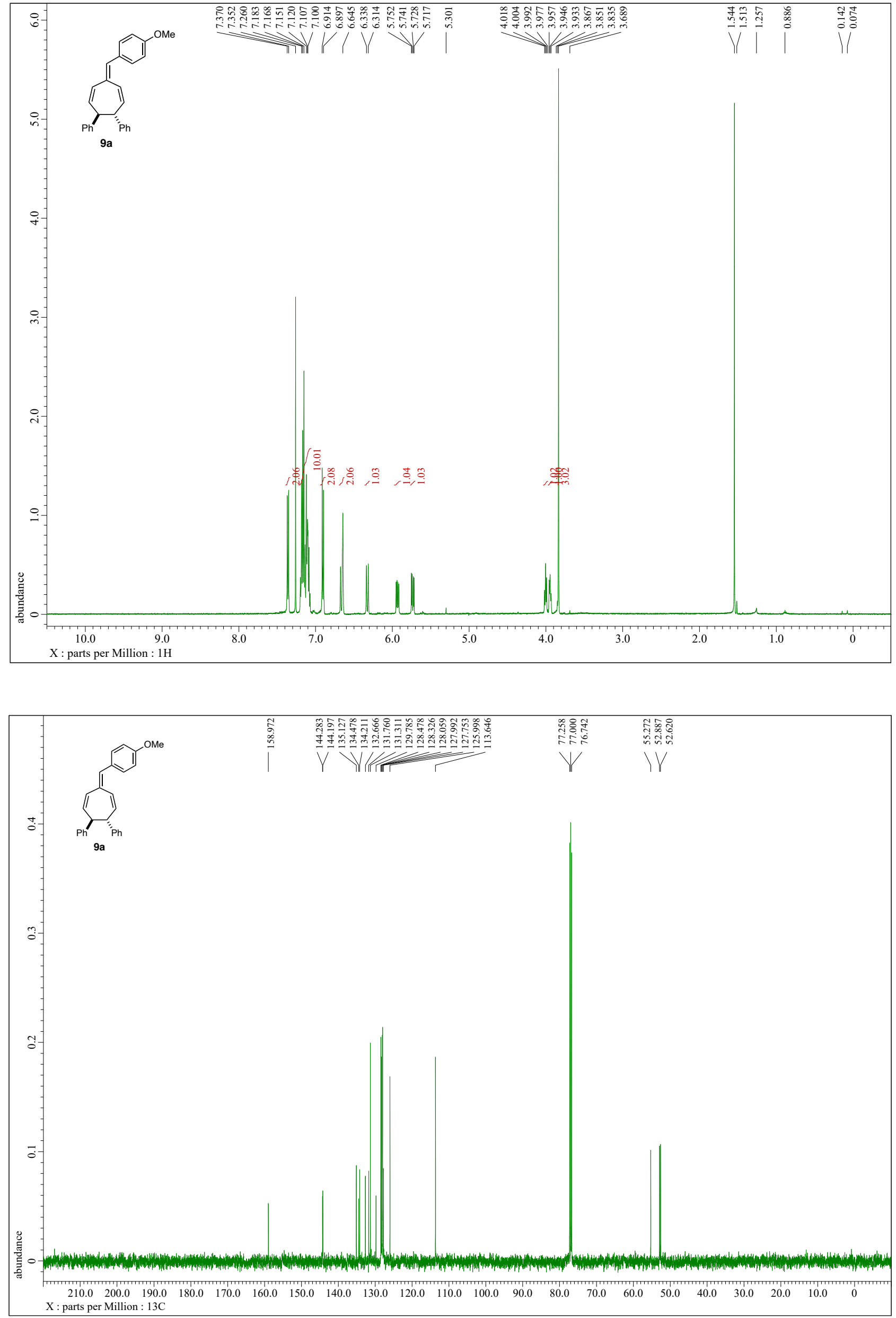
NMR spectra of $9 \mathbf{b}\left[{ }^{1} \mathrm{H}(500 \mathrm{MHz})\right.$ and ${ }^{13} \mathrm{C}(126 \mathrm{MHz})$ in $\left.\mathrm{CDCl}_{3}\right]$
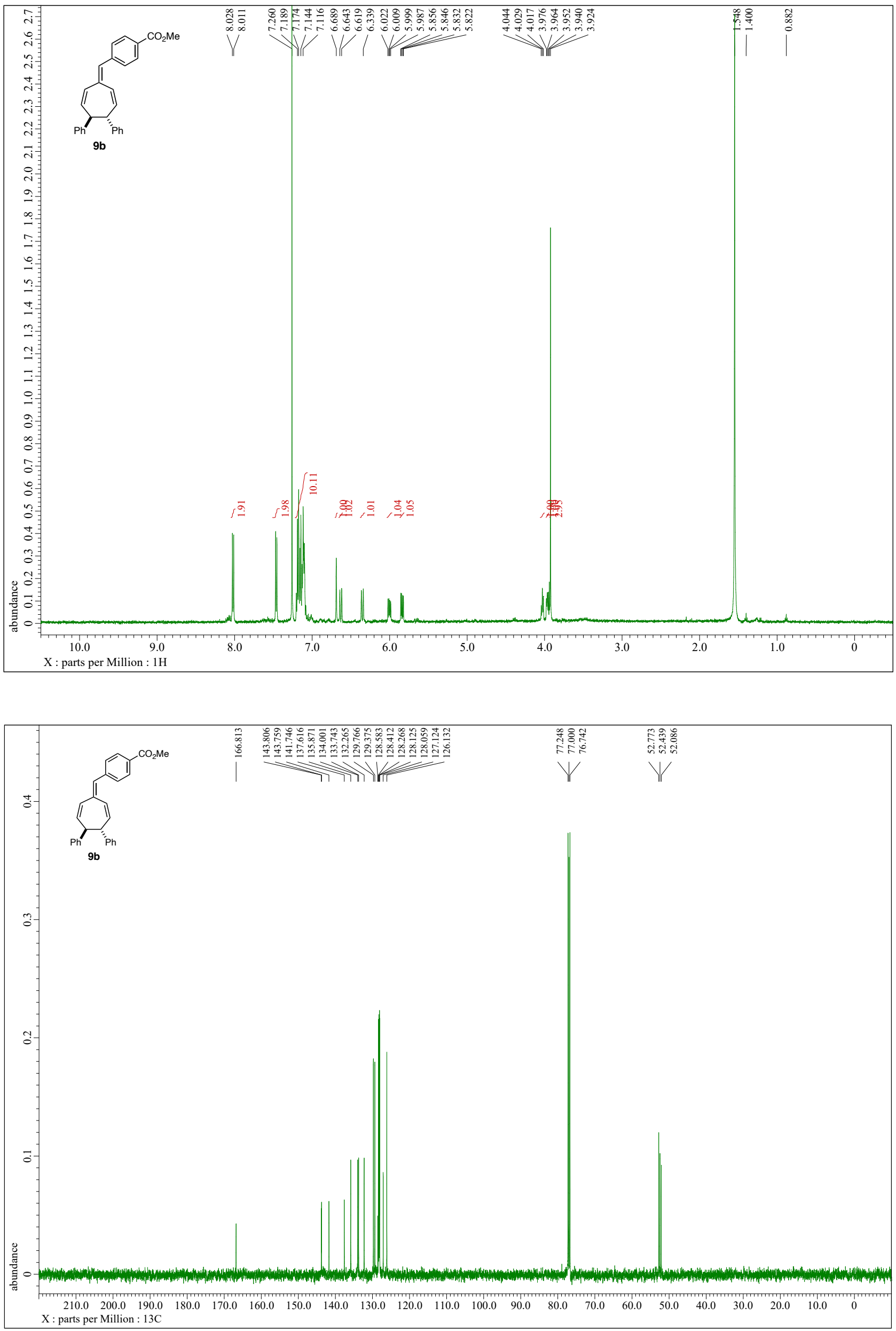
NMR spectra of $9 \mathbf{c}\left[{ }^{1} \mathrm{H}(500 \mathrm{MHz})\right.$ and ${ }^{13} \mathrm{C}(126 \mathrm{MHz})$ in $\left.\mathrm{CDCl}_{3}\right]$
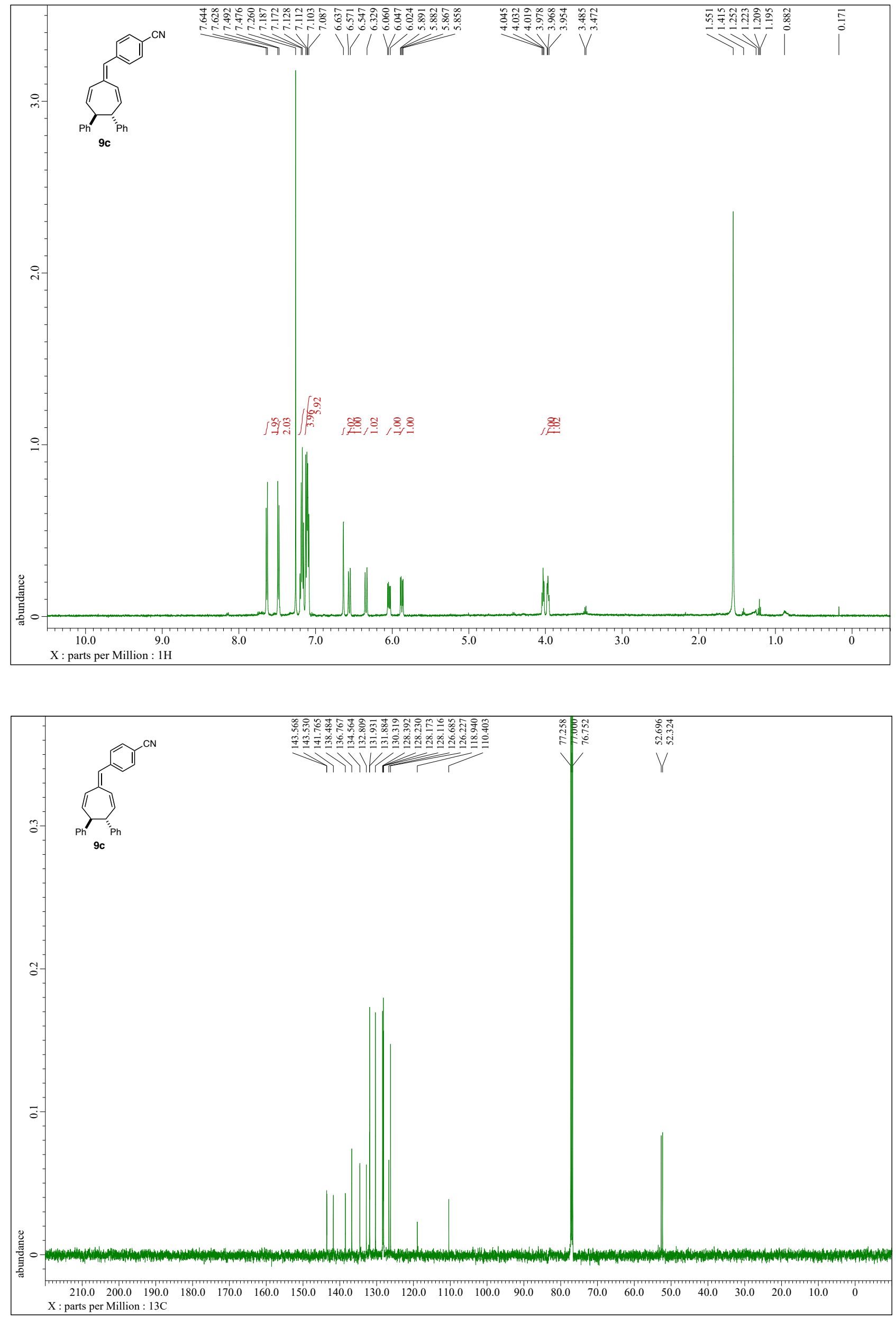
NMR spectra of 9d $\left[{ }^{1} \mathrm{H}(500 \mathrm{MHz})\right.$ and ${ }^{13} \mathrm{C}(126 \mathrm{MHz})$ in $\left.\mathrm{CDCl}_{3}\right]$
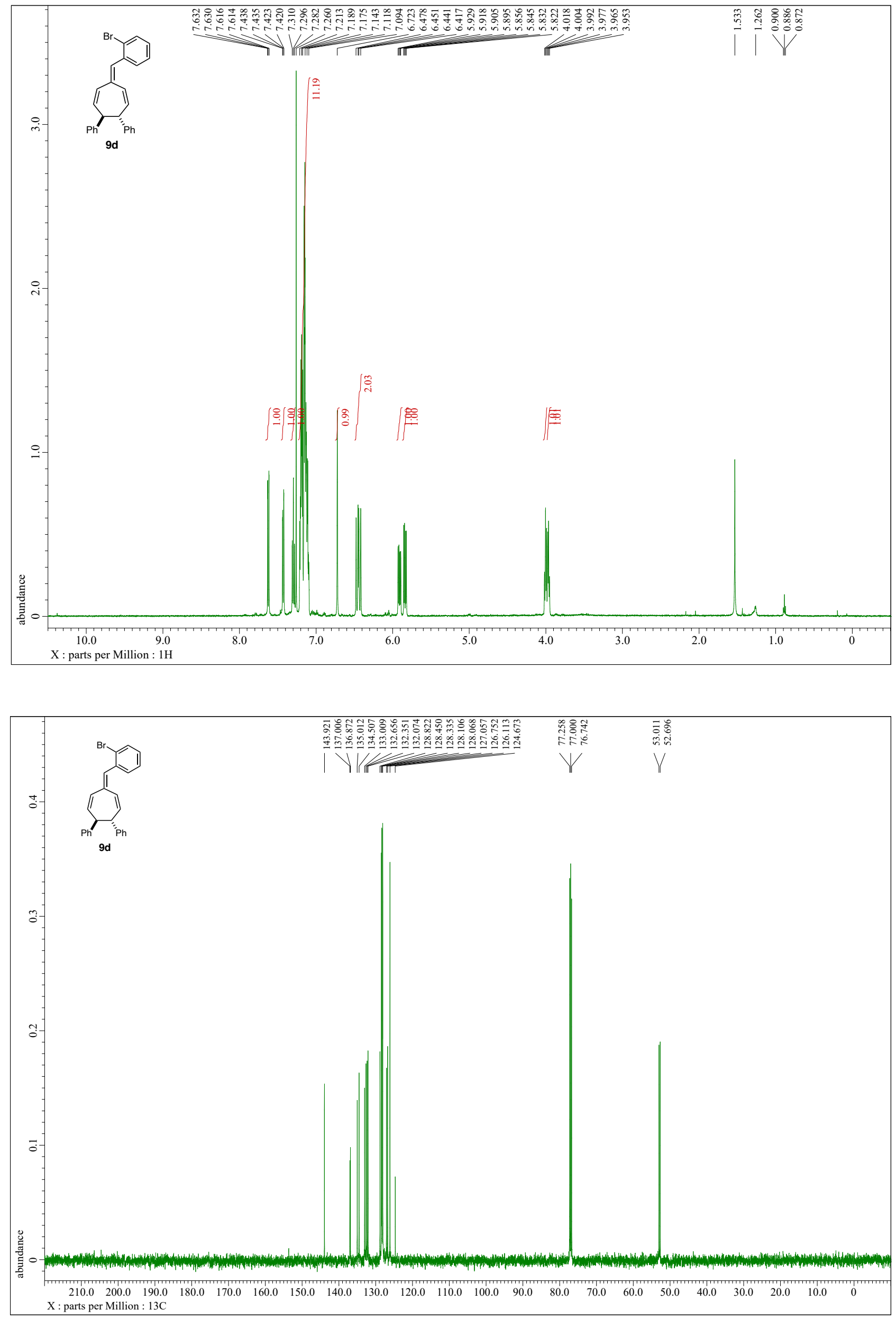
NMR spectra of 9e $\left[{ }^{1} \mathrm{H}(500 \mathrm{MHz})\right.$ and ${ }^{13} \mathrm{C}(126 \mathrm{MHz})$ in $\left.\mathrm{CDCl}_{3}\right]$
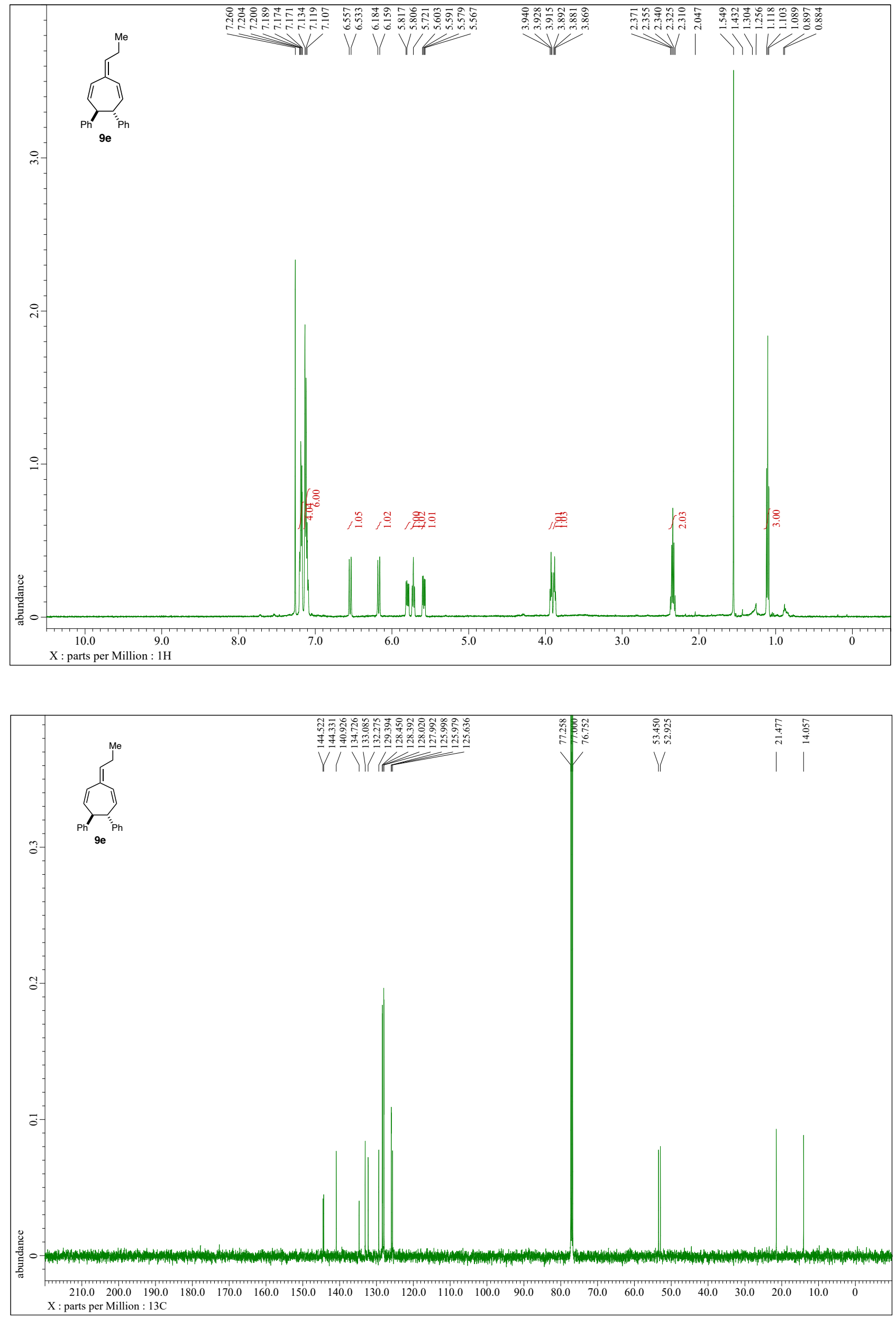
NMR spectra of $9 f\left[{ }^{1} \mathrm{H}(500 \mathrm{MHz})\right.$ and ${ }^{13} \mathrm{C}(126 \mathrm{MHz})$ in $\left.\mathrm{CDCl}_{3}\right]$
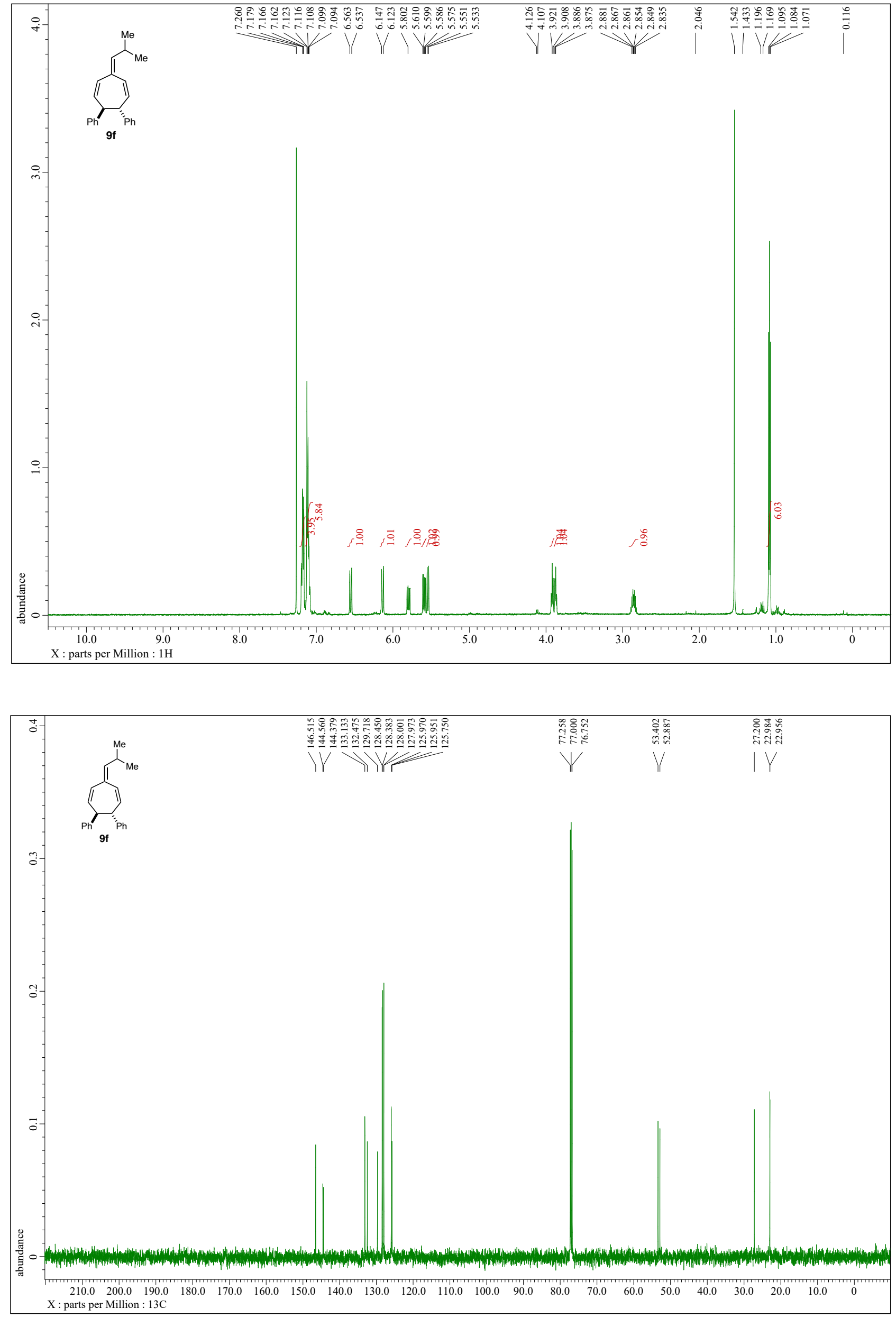
NMR spectra of 11a $\left[{ }^{1} \mathrm{H}(500 \mathrm{MHz})\right.$ and ${ }^{13} \mathrm{C}(126 \mathrm{MHz})$ in $\left.\mathrm{CDCl}_{3}\right]$
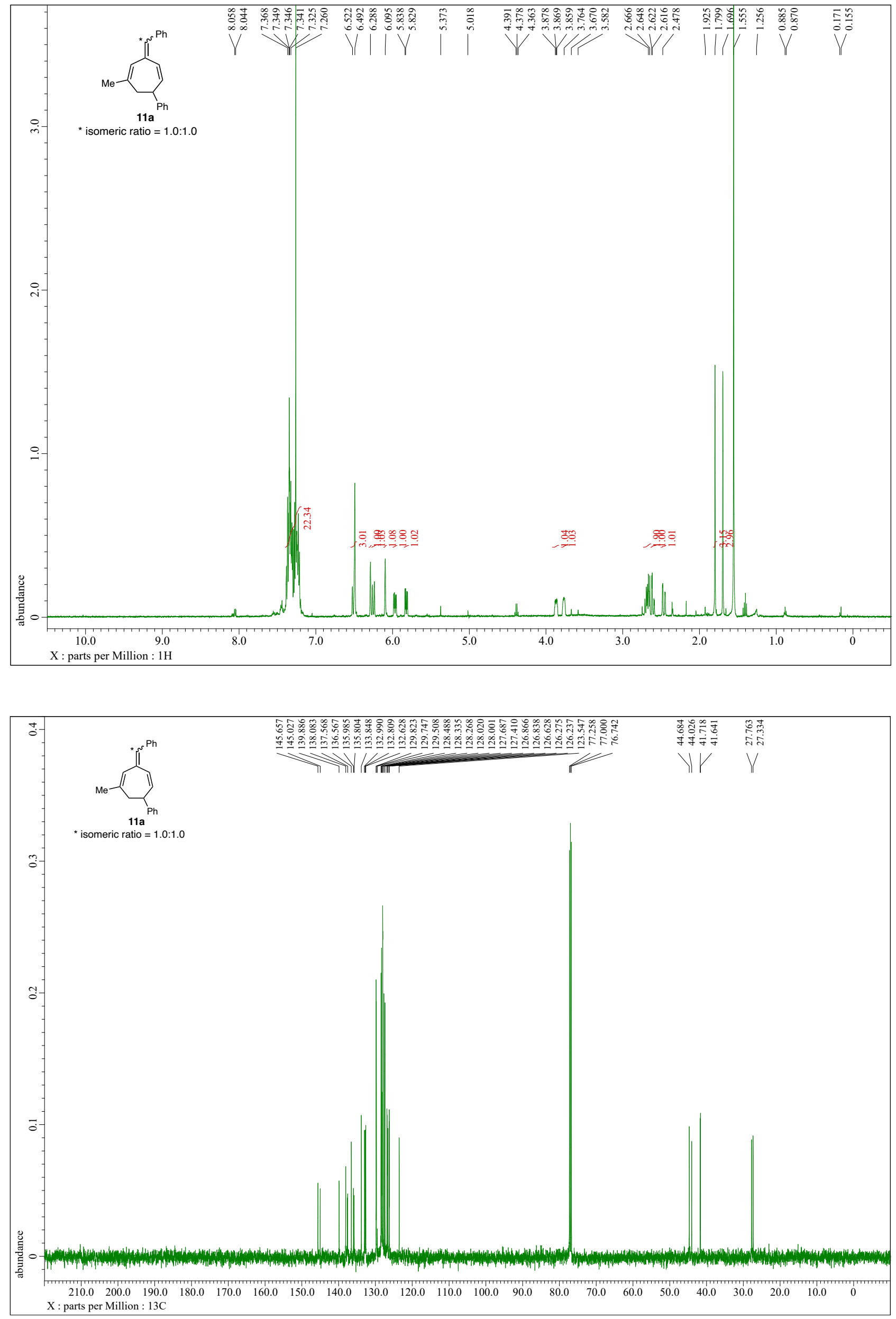
NMR spectra of 11b $\left[{ }^{1} \mathrm{H}(500 \mathrm{MHz})\right.$ and ${ }^{13} \mathrm{C}(126 \mathrm{MHz})$ in $\left.\mathrm{CDCl}_{3}\right]$
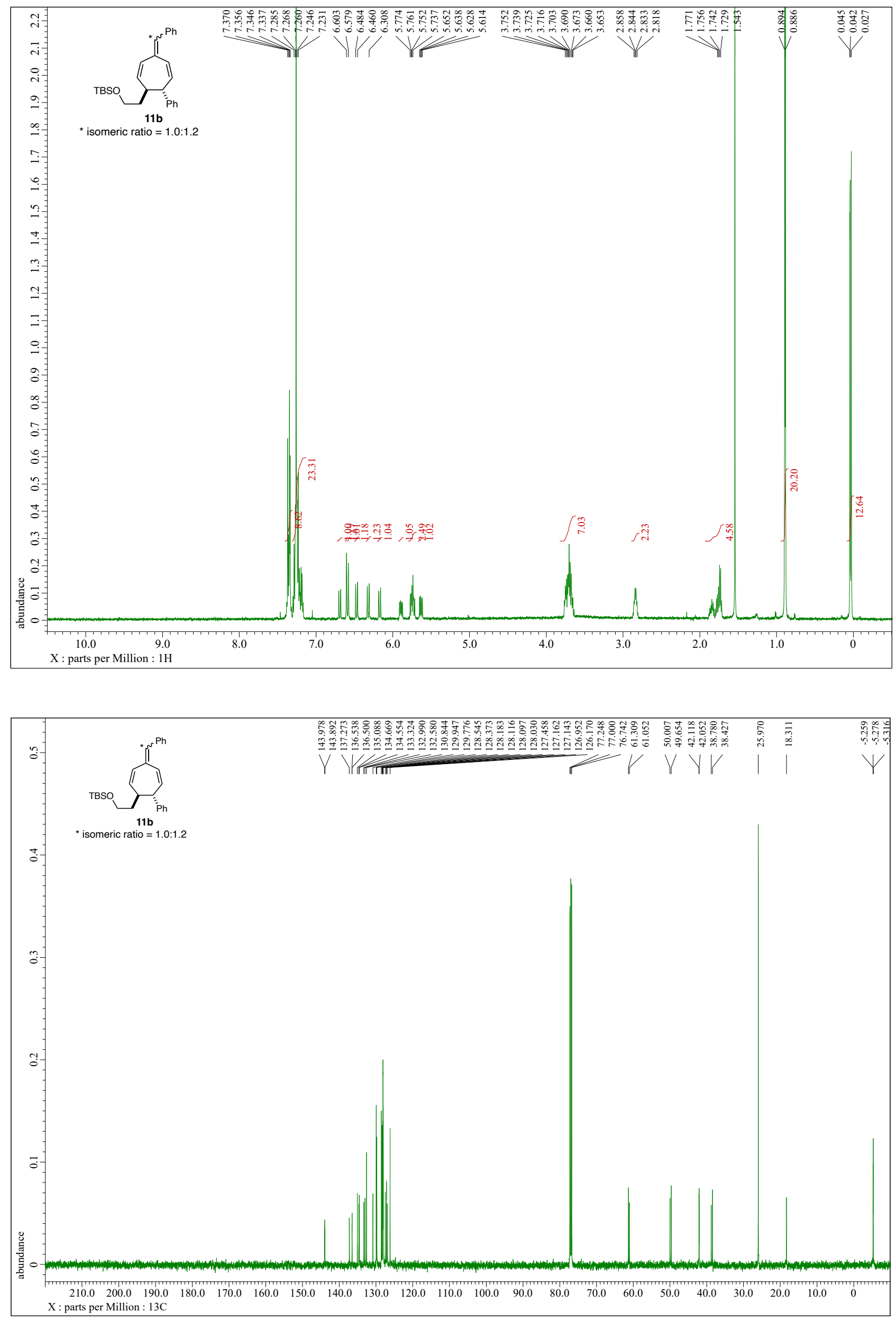
NMR spectra of 11c $\left[{ }^{1} \mathrm{H}(500 \mathrm{MHz})\right.$ and ${ }^{13} \mathrm{C}(126 \mathrm{MHz})$ in $\left.\mathrm{CDCl}_{3}\right]$
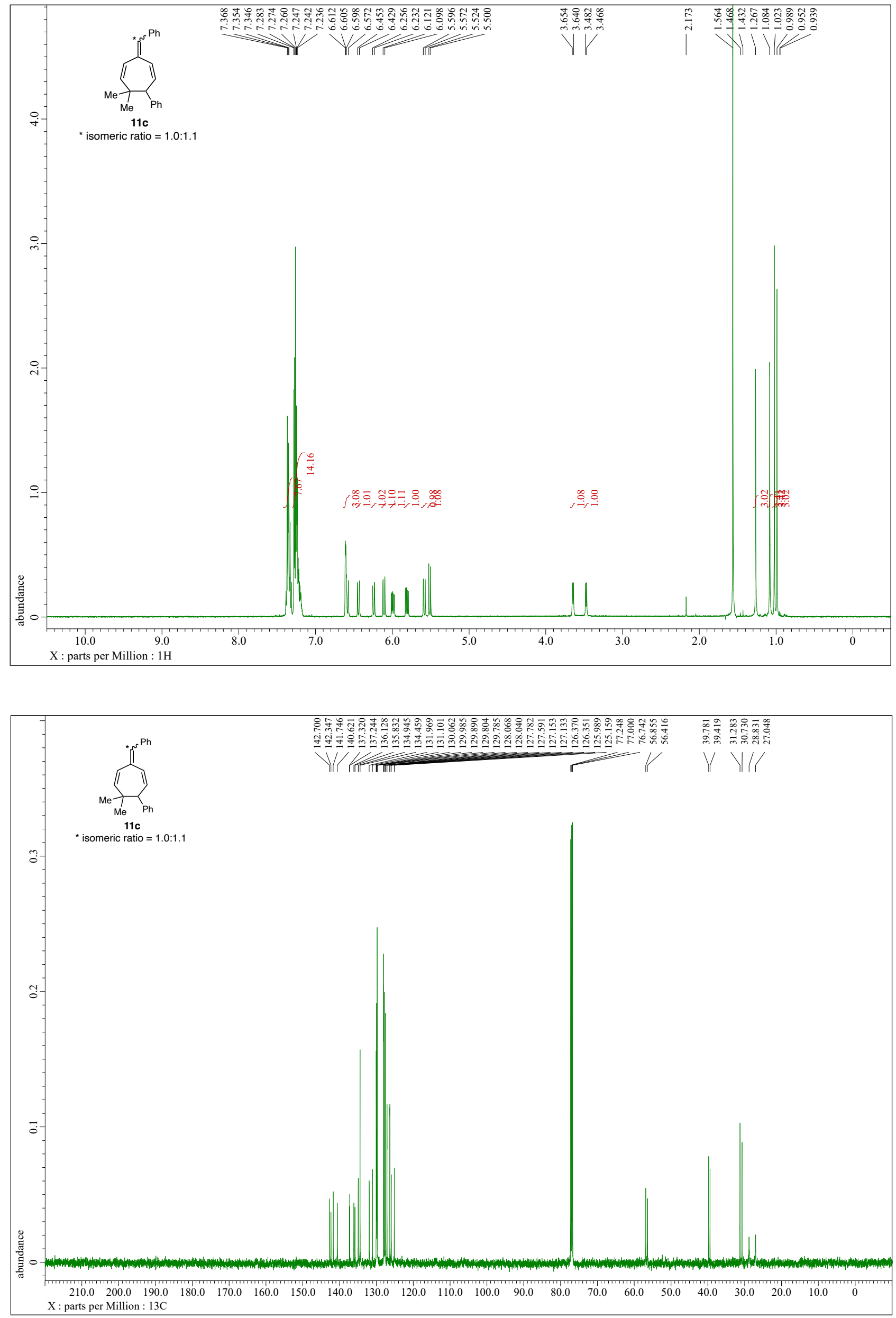
NMR spectra of 11d $\left[{ }^{1} \mathrm{H}(500 \mathrm{MHz})\right.$ and ${ }^{13} \mathrm{C}(126 \mathrm{MHz})$ in $\left.\mathrm{CDCl}_{3}\right]$
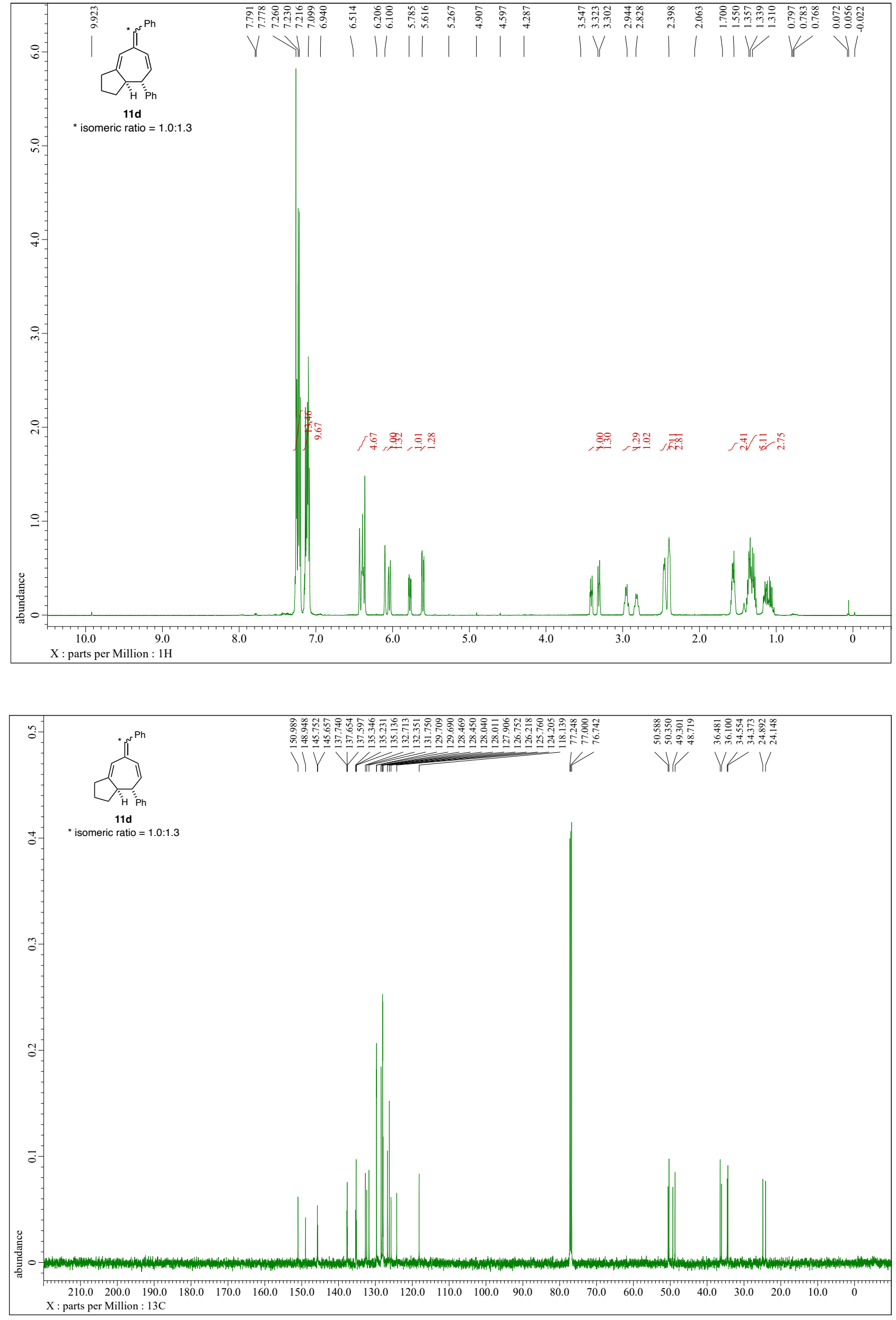
NMR spectra of 11e $\left[{ }^{1} \mathrm{H}(500 \mathrm{MHz})\right.$ and ${ }^{13} \mathrm{C}(126 \mathrm{MHz})$ in $\left.\mathrm{CDCl}_{3}\right]$
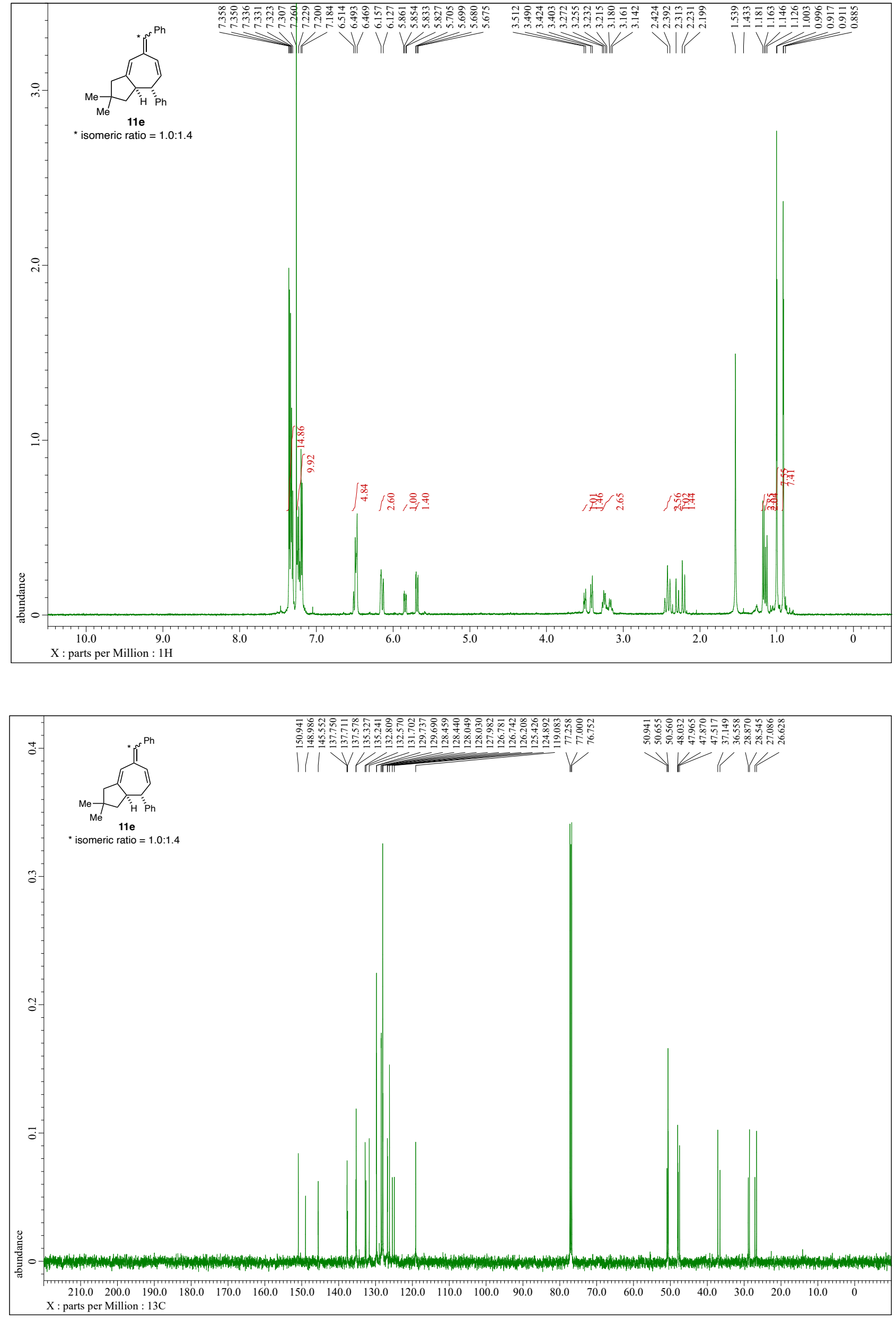
NMR spectra of $11 \mathbf{f}\left[{ }^{1} \mathrm{H}(500 \mathrm{MHz})\right.$ and ${ }^{13} \mathrm{C}(126 \mathrm{MHz})$ in $\left.\mathrm{CDCl}_{3}\right]$
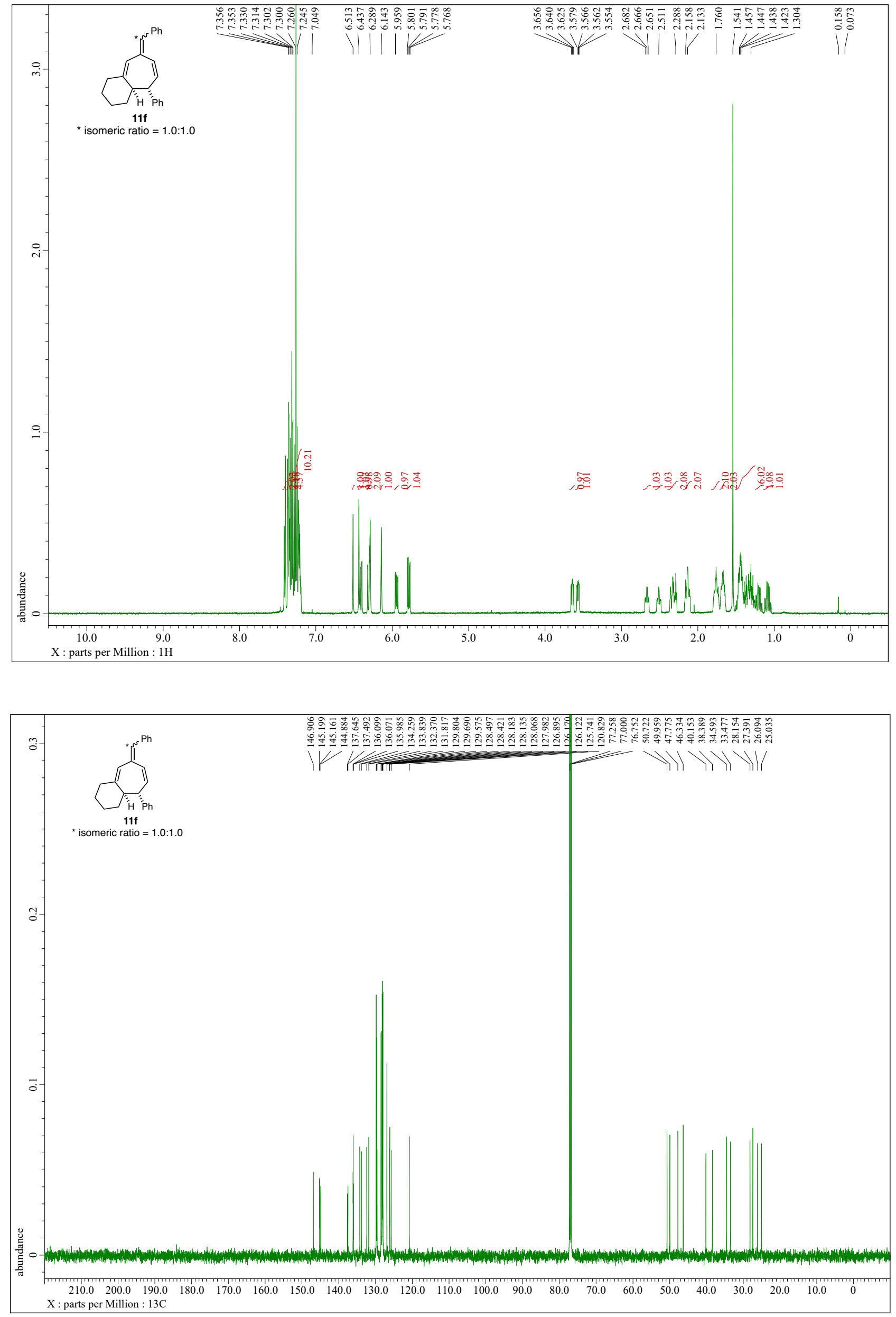
NMR spectra of 13a $\left[{ }^{1} \mathrm{H}(500 \mathrm{MHz})\right.$ and ${ }^{13} \mathrm{C}(126 \mathrm{MHz})$ in $\left.\mathrm{CDCl}_{3}\right]$
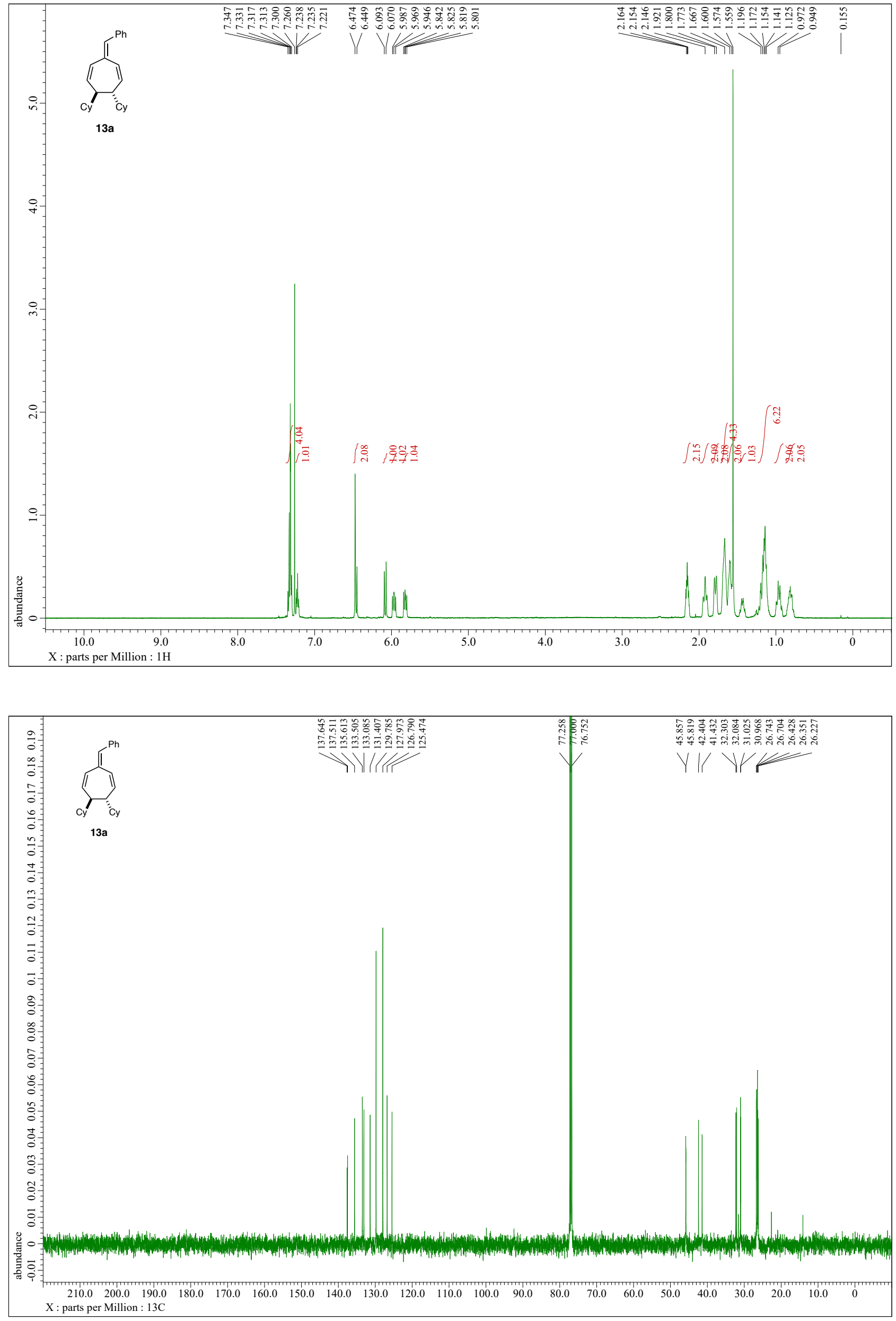
NMR spectra of 13b $\left[{ }^{1} \mathrm{H}(500 \mathrm{MHz})\right.$ and ${ }^{13} \mathrm{C}(126 \mathrm{MHz})$ in $\left.\mathrm{CDCl}_{3}\right]$
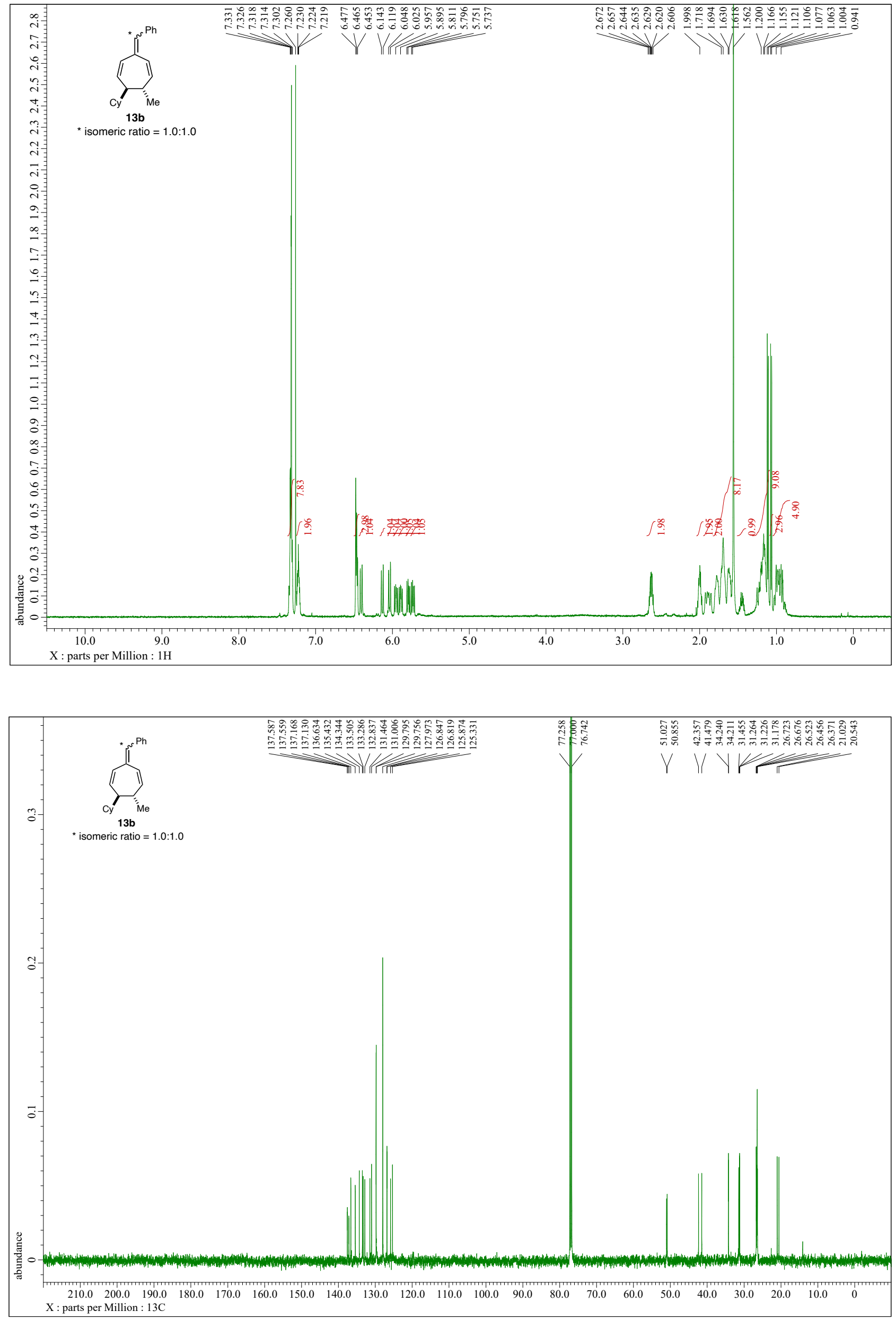
NMR spectra of 13c (13d) $\left[{ }^{1} \mathrm{H}(500 \mathrm{MHz})\right.$ and ${ }^{13} \mathrm{C}(126 \mathrm{MHz})$ in $\left.\mathrm{CDCl}_{3}\right]$
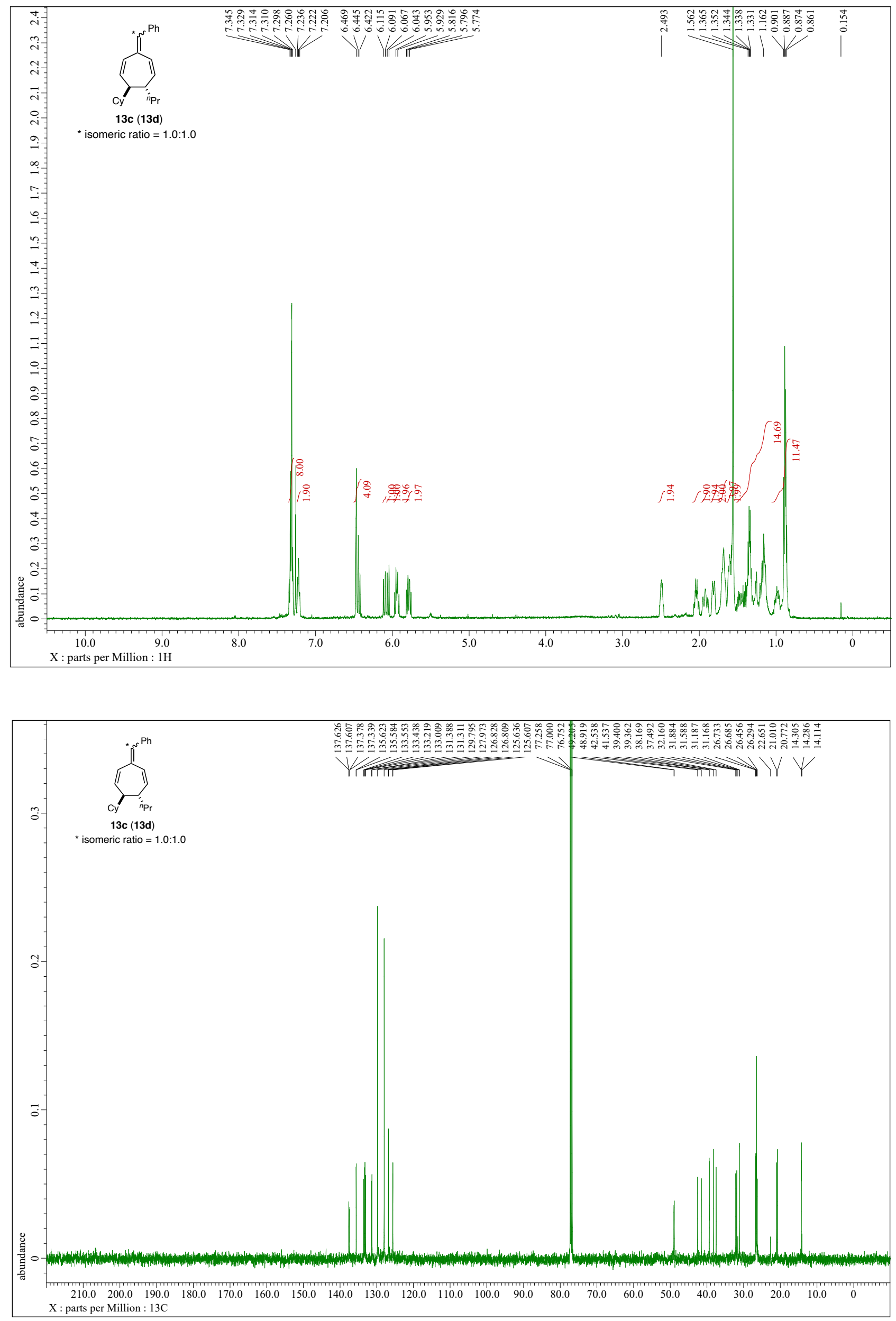
NMR spectra of $\mathbf{1 4}\left[{ }^{1} \mathrm{H}(500 \mathrm{MHz})\right.$ and ${ }^{13} \mathrm{C}(126 \mathrm{MHz})$ in $\left.\mathrm{CDCl}_{3}\right]$
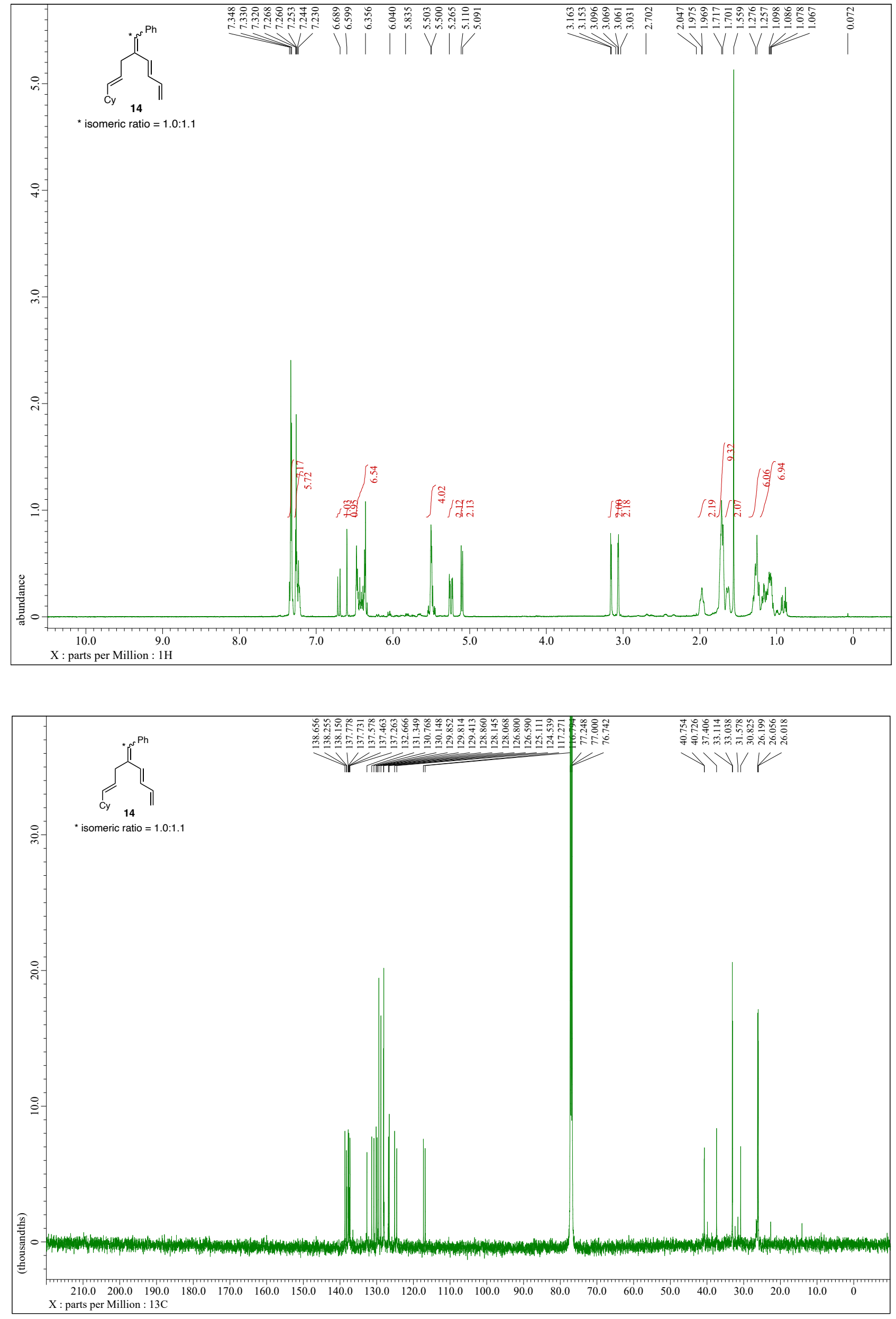
NMR spectra of $16\left[{ }^{1} \mathrm{H}(500 \mathrm{MHz}),{ }^{13} \mathrm{C}(126 \mathrm{MHz})\right.$, and ${ }^{31} \mathrm{P}(202 \mathrm{MHz})$ in $\left.\mathrm{CDCl}_{3}\right]$
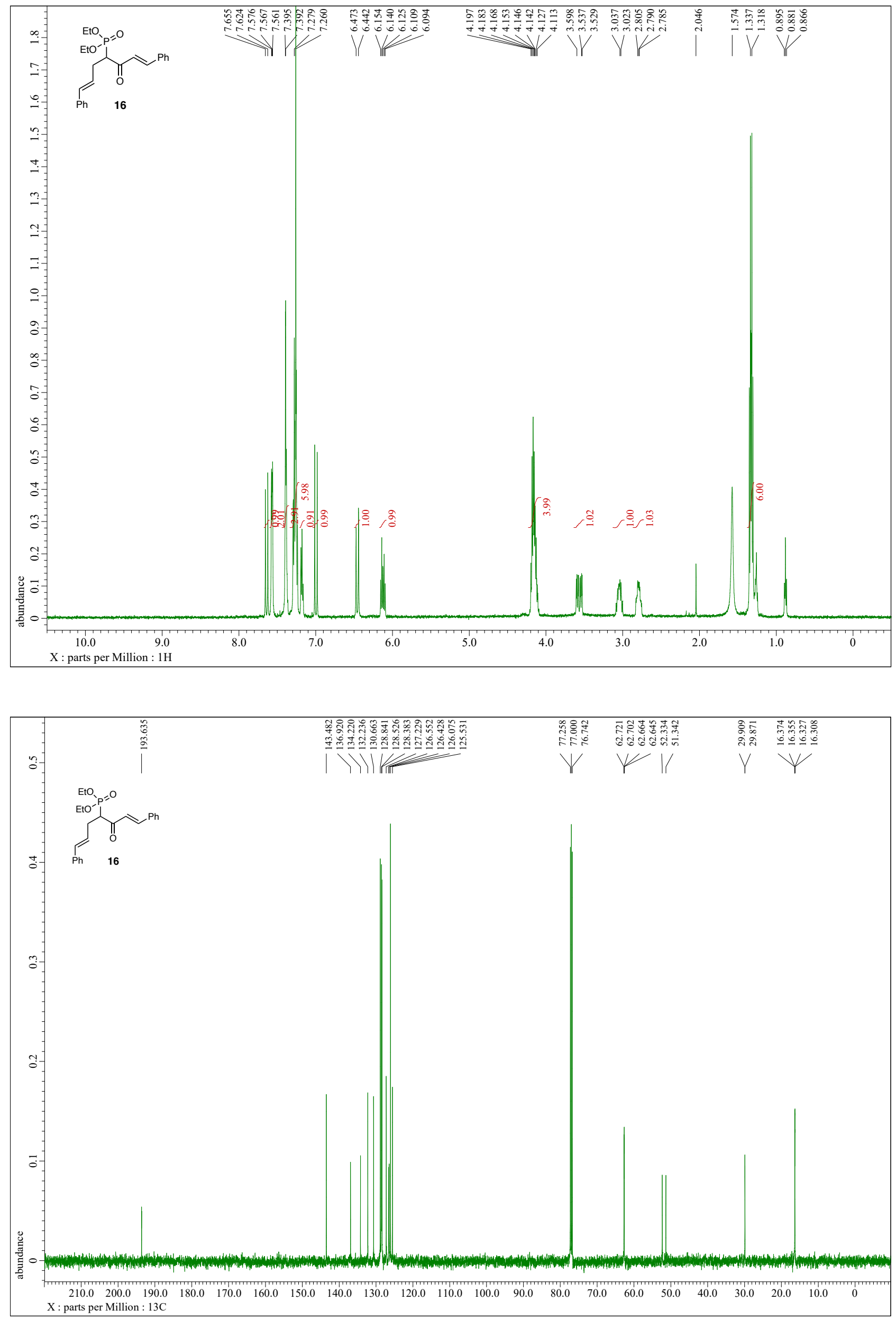


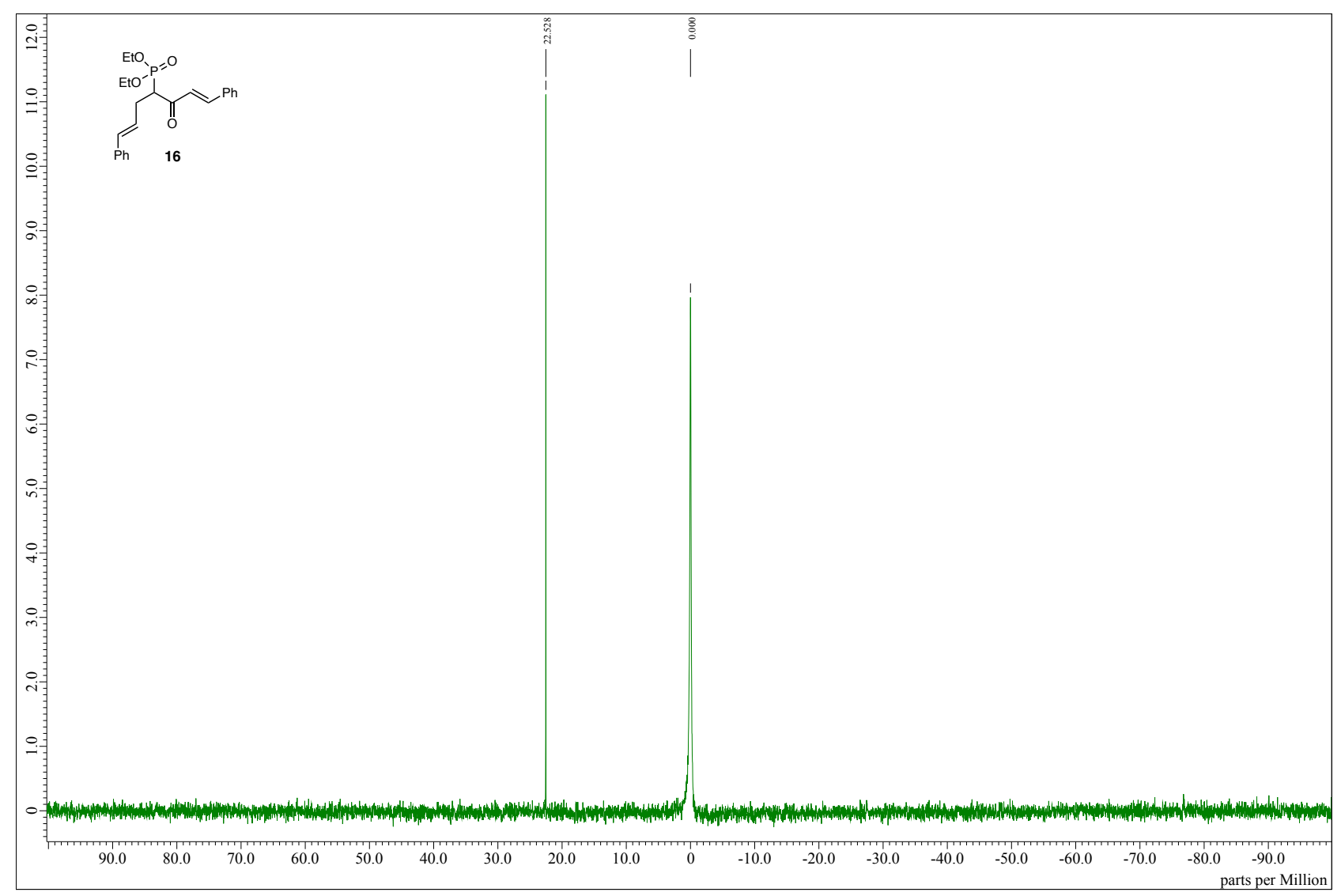


NMR spectra of $\mathbf{1 8}\left[{ }^{1} \mathrm{H}(500 \mathrm{MHz})\right.$ and ${ }^{13} \mathrm{C}(126 \mathrm{MHz})$ in $\left.\mathrm{CDCl}_{3}\right]$
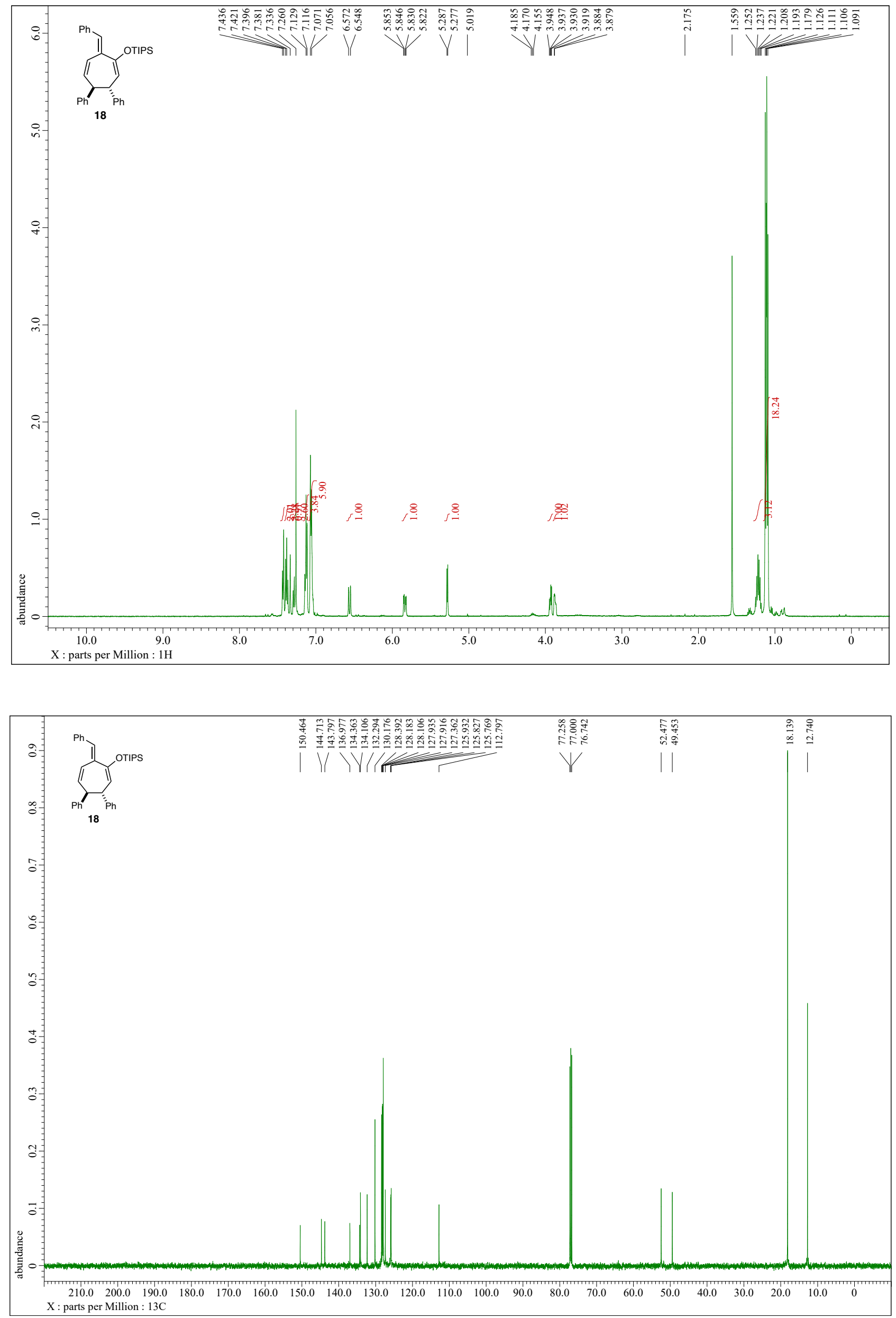
NMR spectra of $19\left[{ }^{1} \mathrm{H}(500 \mathrm{MHz})\right.$ and ${ }^{13} \mathrm{C}(126 \mathrm{MHz})$ in $\left.\mathrm{CDCl}_{3}\right]$
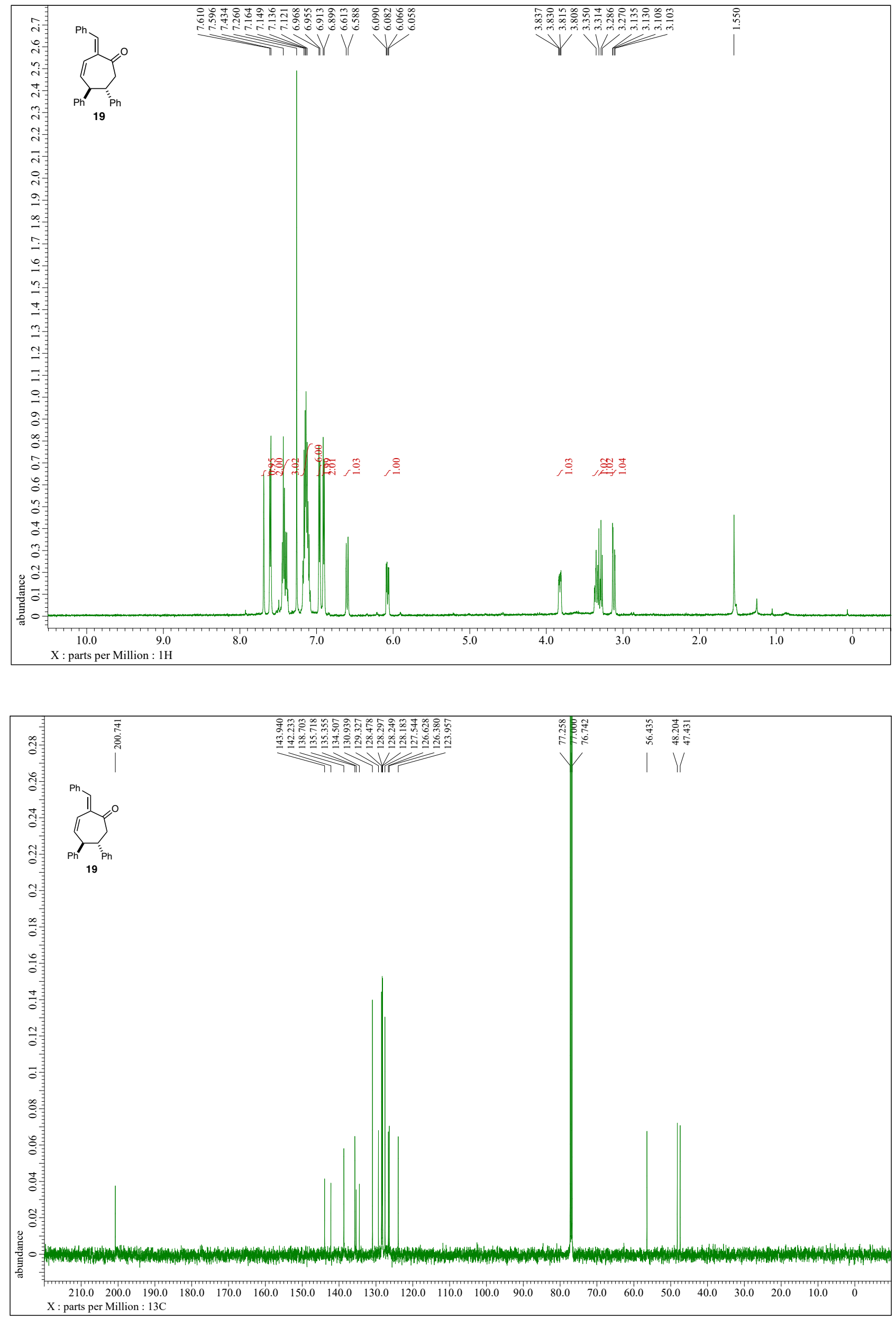

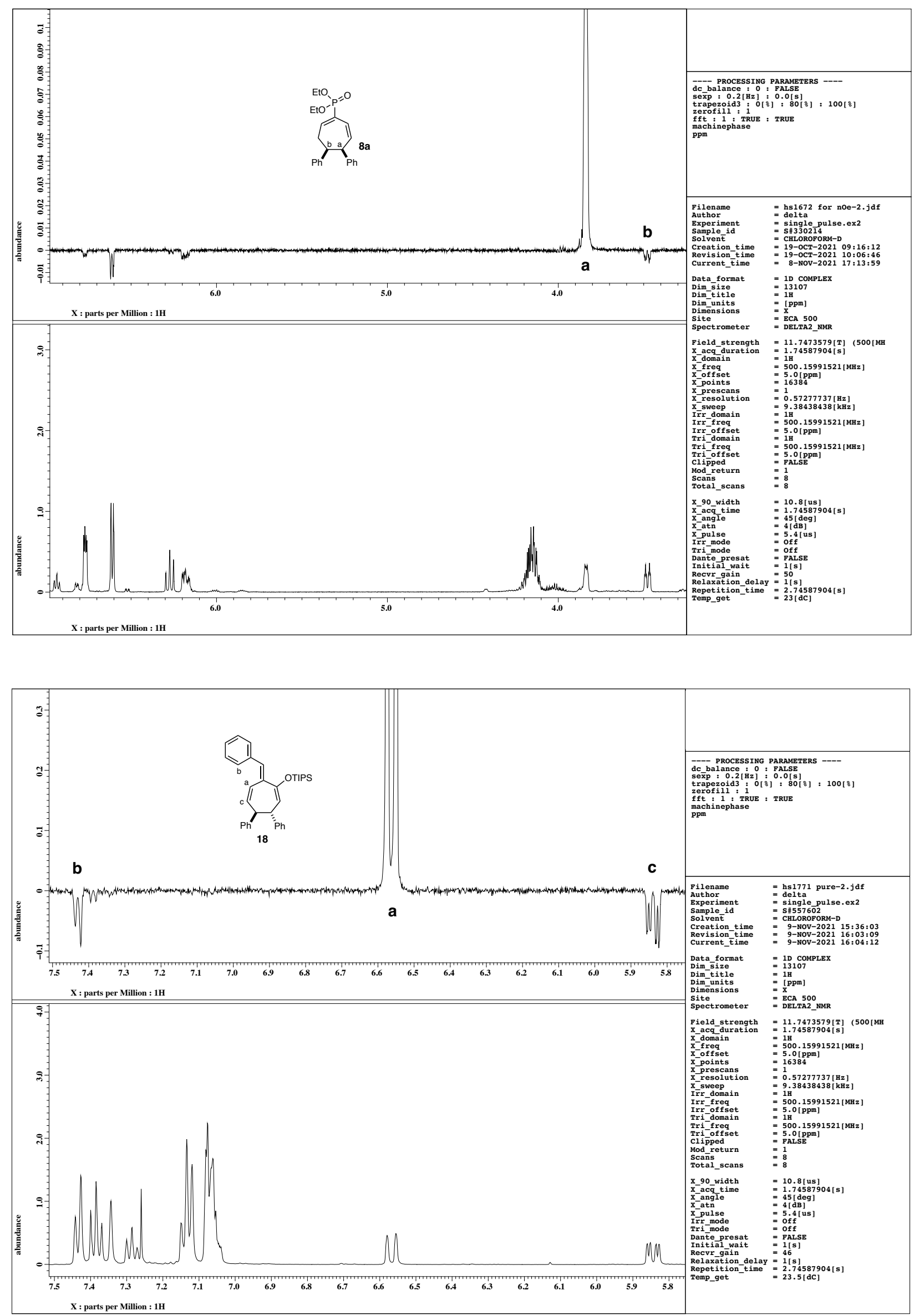

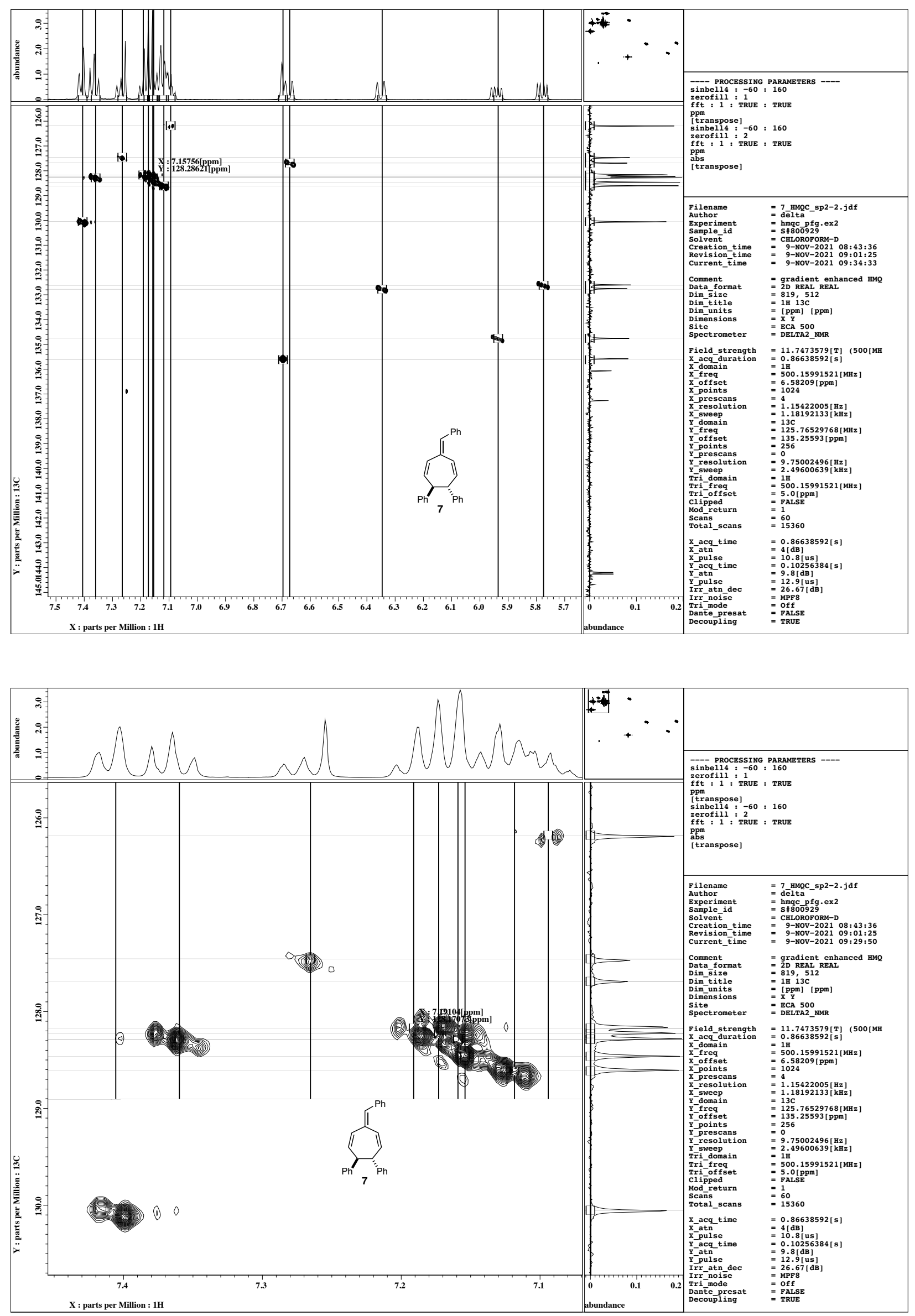